\title{
The interplay of SmNBR1 and SmATG8 in selective autophagy of the filamentous fungus
}

Sordaria macrospora

\author{
Dissertation \\ for the award of the degree \\ "Doctor rerum naturalium" \\ of the Georg-August-Universität Göttingen
}

within the doctoral program "Microbiology and Biochemistry"

of the Georg-August University School of Science (GAUSS)

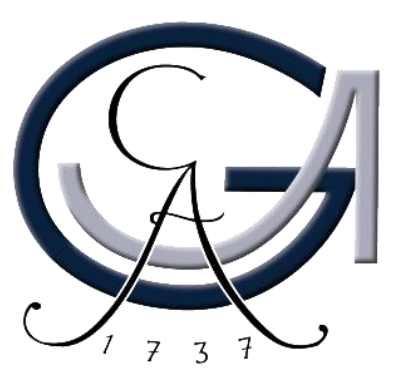

submitted by

Antonia Werner

from Leinefelde

Göttingen, 2017 



\section{Thesis Committee:}

Prof. Dr. Stefanie Pöggeler, Department of Genetics of Eukaryotic Microorganisms, Institute of Microbiology and Genetics

Prof. Dr. Gerhard Braus, Department of Molecular Microbiology and Genetics, Institute of Microbiology and Genetics

Prof. Dr. Michael Thumm, Department of Cellular Biochemistry, University Medical Center Göttingen

\section{Members of the Examination Board:}

Referee: Prof. Dr. Stefanie Pöggeler, Department of Genetics of Eukaryotic Microorganisms, Institute of Microbiology and Genetics

$2^{\text {nd }}$ Referee: Prof. Dr. Gerhard Braus, Department of Molecular Microbiology and Genetics, Institute of Microbiology and Genetics

\section{Further members of the Examination Board:}

Prof. Dr. Michael Thumm, Department of Cellular Biochemistry, University Medical Center Göttingen

Prof. Dr. Heike Krebber, Department of Molecular Genetics, Institute of Microbiology and Genetics

Jun. Prof. Dr. Kai Heimel, Department of Molecular Microbiology and Genetics, Institute of Microbiology and Genetics

PD Dr. Fabian Commichau, Department of General Microbiology, Institute for Microbiology and Genetics

Date of oral examination: 28.03.2017 


\section{Affirmation}

I hereby declare that this thesis was written independently and with no other sources and aids than quoted.

Göttingen, 02.02.2017

Antonia Werner 


\section{Publication based on this thesis}

This doctoral study was performed in the group of Prof. Dr. Stefanie Pöggeler in the Department of Genetics of Eukaryotic Microorganisms at the Institute of Microbiology and Genetics, Georg-August University Göttingen.

Some parts of the results section of this doctoral study were peer-reviewed and published in the journal "PLOS ONE".

Antonia Werner, Britta Herzog, Stefan Frey and Stefanie Pöggeler (2016). Autophagyassociated protein SmATG12 is required for fruiting-body formation in the filamentous ascomycete Sordaria macrospora. PLOS ONE/DOI: 10.1371/journal.pone.0157960. Accepted manuscript online: 16. June 2016.

Author contributions to this publication:

Planned experiments: AW, SP, BH, SF

Performed experiments: AW, BH, SF

Analyzed data: AW, SP

Contributed reagents or other essential material: SP

Wrote the paper: $\mathrm{AW}, \mathrm{BH}, \mathrm{SP}$ 



\section{Table of contents}

I. Table of contents i

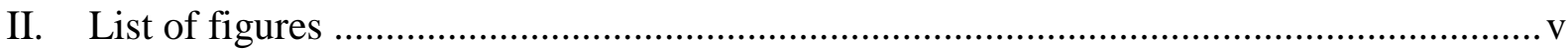

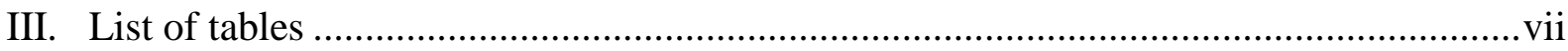

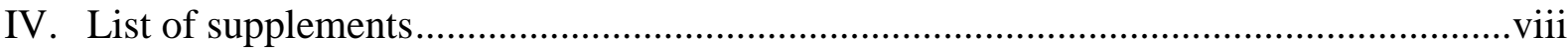

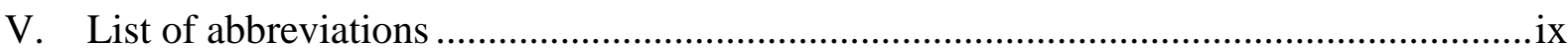

Summary

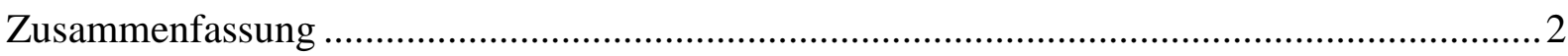

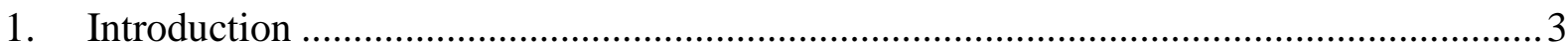

1.1 Overview of autophagy in eukaryotes ..................................................................3

1.2 Macroautophagy in the yeast $S$. cerevisiae ............................................................4

1.3 Selective autophagy processes mediated by cargo receptors.....................................8

1.4 Pexophagy, the autophagic degradation of peroxisomes ........................................ 12

1.5 Ribophagy, the autophagic degradation of ribosomes ............................................ 15

1.6 Sordaria macrospora: a model organism to study fruiting-body development.16

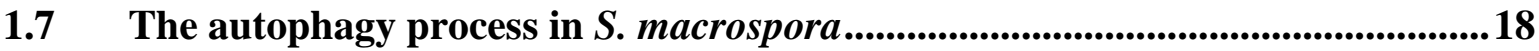

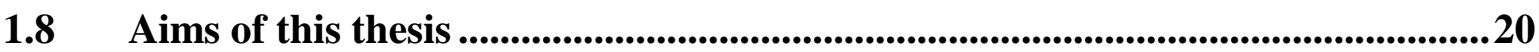

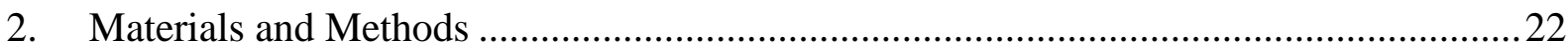

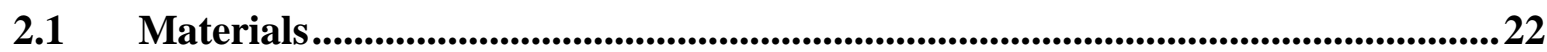

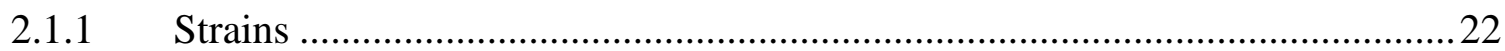

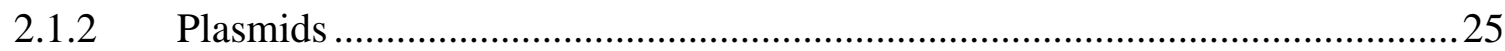

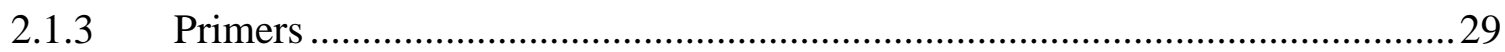

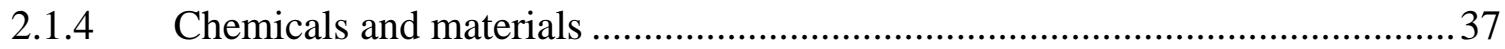

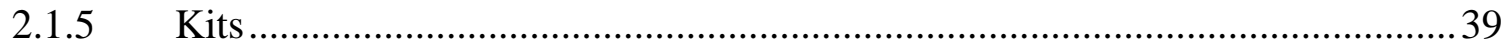

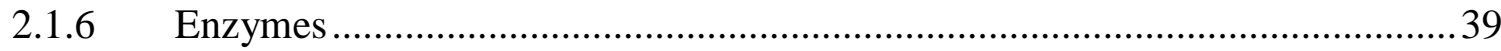

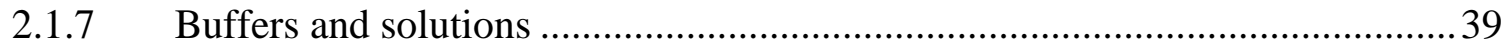

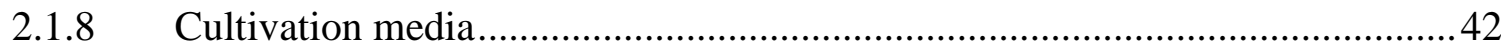

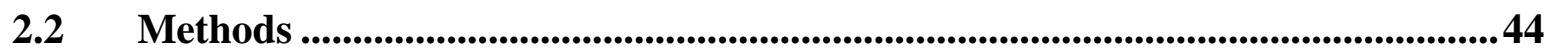

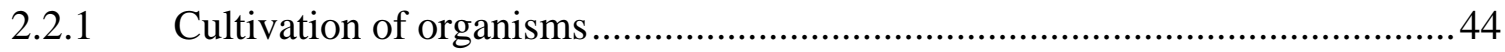


2.2.2 Preparation and transformation of competent microorganisms ...................... 45

2.2.3 Construction of single-spore isolates of $S$. macrospora ............................... 46

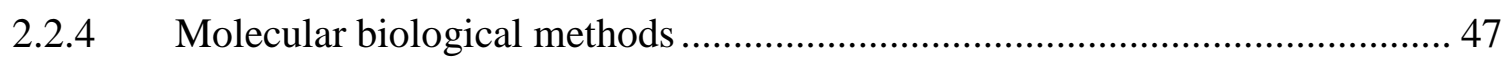

2.2.5 Plasmid construction using homologous recombination in yeast ................... 51

2.2.6 Generation of $S$. macrospora deletion strains ............................................... 51

2.2.7 Plasmid construction and localization analysis of fluorescence-tagged

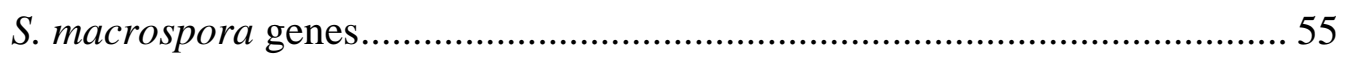

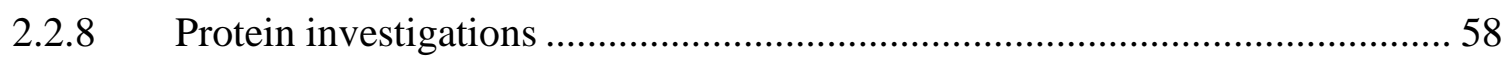

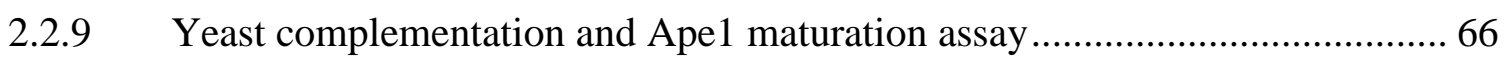

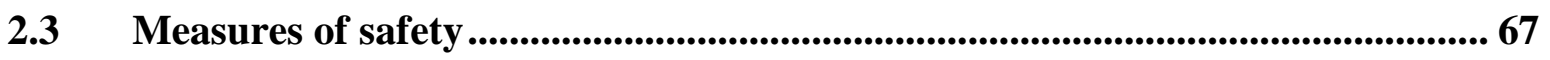

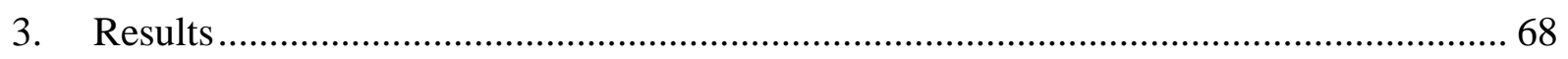

3.1 Characterization of the autophagy-associated protein SmATG12 in

S. macrospora ...........................................................................................................6 68

3.1.1 Identification of a S. macrospora ATG12 homolog as an interaction partner of

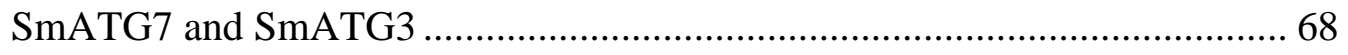

3.1.2 Deletion of Smatg12 impairs vegetative growth and fruiting-body development 73

3.1.3 EGFP-SmATG12 localizes to phagophore assembly sites ............................ 76

\subsection{Identification of the selective autophagy cargo receptor $\mathrm{SmNBR1}$.................. 79}

3.2.1 The cargo receptor SmNBR1 of S. macrospora is conserved in higher eukaryotes and displays a similar domain architecture .................................................. 79

3.2.2 The Smnbrl deletion mutant displays developmental defects ....................... 81

3.2.3 Stress conditions enhance developmental defects of $\Delta$ Smnbr1 ….................. 86

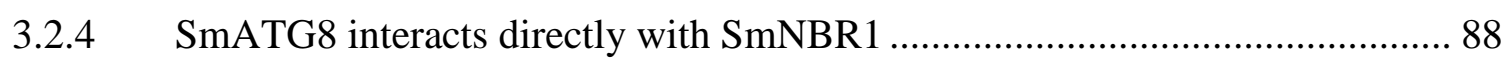

3.2.5 SmNBR1 localizes in autophagosome-like structures and vacuoles............... 90

3.2.6 SmNBR1 displays many putative phosphorylation sites .............................. 95

3.2.7 Overexpression of SmNBR1 has no phenotypical effects, but modifications of SmNBR1 prevent complementation of $\Delta$ Smnbr1 and lead to mislocalization 96

3.3 SmNBR1-dependent pexophagy in S. macrospora ....................................... 98 
3.3.1 $\Delta$ Smnbr1 fails to grow on fatty-acid and $\mathrm{H}_{2} \mathrm{O}_{2}$-containing medium 98

3.3.2 Autophagic degradation of the peroxisomal reporter protein DsRED-SKL arrests in $\Delta$ Smnbr 1

3.3.3 Peroxisomes change in size, number and morphology in $\Delta \operatorname{Smnbr1}$ and $\Delta \operatorname{Smatg} 8$ mutants 101

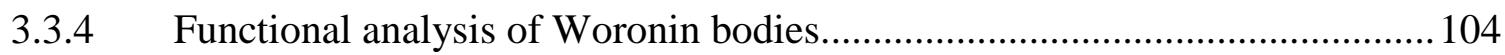

3.4 SmNBR1-dependent ribophagy in S. macrospora .......................................106

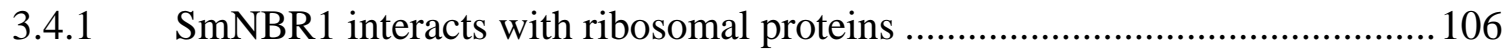

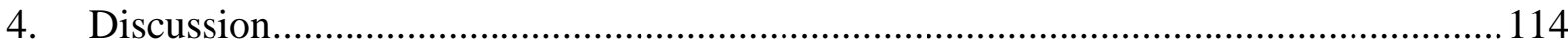

\subsection{SmATG12, a core autophagy protein ............................................................114}

4.1.1 The conserved autophagy-associated protein SmATG12 is unable to rescue the S. cerevisiae atg $12 \Delta$ mutant

4.1.2 SmATG12 is required for sexual reproduction and normal vegetative growth

4.1.3 Deletion of Smatg12 abolishes delivery of EGFP-labeled SmATG8 to the vacuole

4.2 The selective autophagy cargo receptor SmNBR1

4.2.1 SmNBR1 has a conserved domain architecture

4.2.2 SmNBR1 and SmATG8 are direct interaction partners which interact via the LIR motif of SmNBR1

4.2.3 The S. macrospora SmNBR1 is required for proper sexual development, vegetative growth and stress resistance.....

4.2.4 SmNBR1 has many putative phosphorylation sites

4.2.5 In S. macrospora, pexophagy depends on SmNBR1.

4.2.6 Woronin bodies are functional in autophagy-defective mutants

4.2.7 SmNBR1 is involved in selective degradation of the large ribosomal subunit

5. References

6. Appendix 


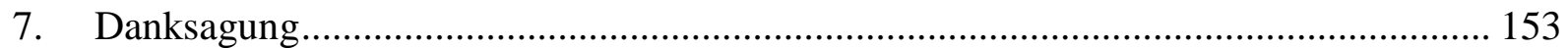

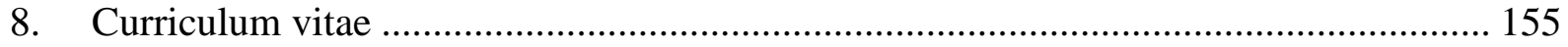




\section{List of figures}

Figure 1: Overview of autophagy processes.

Figure 2: Five sequential steps of autophagy in S. cerevisiae ............................................5

Figure 3: Proteins involved in autophagy in the yeast $S$. cerevisiae .....................................

Figure 4: Cargo recognition during selective autophagy................................................. 10

Figure 5: Domain architecture of the Homo sapiens NBR1 and p62 ................................... 11

Figure 6: Model for the role of Ltn1 and Ubp3/Bre5 in starvation-induced ribophagy in

S. cerevisiae (modified after Ossareh-Nazari et al. (2014)). ......................................... 15

Figure 7: Life cycle of the homothallic ascomycete $S$. macrospora (Kück et al. 2009)......... 17

Figure 8: Multiple-sequence alignment of ATG12 orthologs from fungi, plants and animals.69 Figure 9: Yeast-two hybrid interaction of SmATG12 with SmATG7, SmATG3 and SmATG8.

Figure 10: Verification of the protein expression of SmATG12 and SmATG8 in yeast-two

hybrid transformants. .71

Figure 11: Complementation of the $S$. cerevisiae $\operatorname{atg} 12 \Delta$ autophagy mutant with the

S. macrospora Smatg12 gene. .72

Figure 12: Proteolytic cleavage of GFP-ScATG8. .72

Figure 13: Construction of a $\Delta \operatorname{Smatg} 12$ mutant.

Figure 14: EGFP-SmATG8 protein degradation in the $\Delta$ Smatg12 strain compared to the corresponding complemented $\Delta$ Smatg8::egfp-Smatg8 ${ }^{\text {ect }}$ strain.

Figure 15: Phenotypic characterization of S. macrospora wt, Smatg12 deletion and complementation strain. 75

Figure 16: Fluorescence microscopic localization of EGFP-SmATG12 and EGFP-SmATG8.

Figure 17: Unrooted phylogenetic neighbor-joining tree of NBR1 homologs from 20 species.

Figure 18: Schematic representation of cargo receptor homologs and specific domains. ....... 80

Figure 19: Generation and analysis of a $\Delta$ Smnbr1 knockout strain. 82

Figure 20: Phenotypic analysis of $S$. macrospora $\mathrm{wt}, \Delta$ Smnbr1 mutant strain and complementation strains expressing S. macrospora or H. sapiens NBR1 proteins. .84

Figure 21: Western blot using anti-ubiquitin to detect ubiquitinated proteins. 86

Figure 22: Vegetative growth and development analysis of $S$. macrospora wt, $\Delta$ Smnbr1 and the complemented mutant on different medium. 
Figure 23: Interaction studies with SmNBR1 and SmATG8. 89

Figure 24: Localization of the autophagy proteins SmNBR1 and SmATG8.

Figure 25: Fluorescence microscopic analysis of $S$. macrospora SmNBR1 and SmATG8 in different deletion mutants.

Figure 26: Quantitative Western experiments of the vacuolar degradation of EGFP-SmATG8 or SmNBR1-DsRED in different autophagy mutants................................................... 94

Figure 27: SmNBR1-protein phosphorylation assay. 95

Figure 28: Phenotypical analysis and fluorescence localization studies of S. macrospora

$\Delta$ Smnbr1 strain transformed with Smnbrl variants under normal, low temperature and autophagy-inducing conditions.

Figure 29: Growth analysis of wt, $\Delta$ Smnbr1 and complementation strain under peroxisomeproliferation or oxidative-stress conditions

Figure 30: Localization studies of the peroxisomal reporter protein DsRED-SKL in wt and $\Delta$ Smnbr1

Figure 31: Microscopic and quantitative investigations of proliferation of SmPEX14 EGFP-

labeled peroxisomes

Figure 32: Co-localization studies of SmPEX14-EGFP clusters and SmNBR1-DsRED...... 103

Figure 33: Localization studies of DsRED-SmHEX1 in wt and autophagy mutants. ........... 105

Figure 34: Degradation of SmRPL25-EGFP under normal and starvation conditions.......... 108

Figure 35: Degradation of SmRPS19-EGFP under normal and starvation conditions.......... 109

Figure 36: EGFP fluorescence localization in wt and $\Delta$ Smnbr1. 110

Figure 37: Quantitative Western-blot analysis of the autophagosomal reporter protein EGFPSmATG8.

Figure 38: Immuniprecipitation of SmNBR1 together with ribosomal proteins.

Figure 39: Mechanistic views of receptor-dependent pexophagy pathways.

Figure 40: Hypothetical model for the ribophagy of the large ribosomal subunit in

S. cerevisiae and S. macrospora.

Figure 41: Crystal structure of the 80S human ribosome and ribosomal RNA (Anger et al.

2013). 


\section{List of tables}

Table 1: Conservation of atg genes in $S$. macrospora compared to yeasts............................ 18

Table 2: Overview of strains used and constructed in this study. ......................................22

Table 3: Overview of the used and generated plasmids in this study...................................25

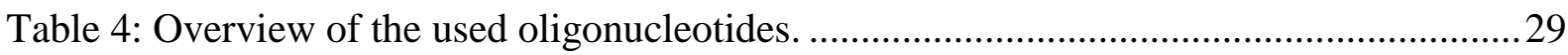

Table 5: Composition of a $50 \mu 1$ PCR reaction for Phusion-or MolTaq polymerase............... 49

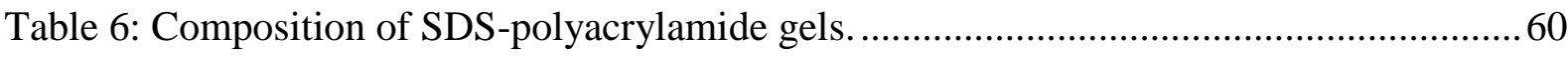

Table 7: Results of GFP-Trap and LC/MS analysis using SmNBR1-EGFP as bait protein.. 106 


\section{List of supplements}

Supplement 1: Multiple-sequence alignment of ATG5 orthologs from Homo sapiens,

Saccharomyces cerevisiae and Sordaria macrospora. 145

Supplement 2: Multiple-sequence alignment of ATG3 orthologs from Sordaria macrospora,

Saccharomyces cerevisiae and Homo sapiens. 146

Supplement 3: Alignment of single domains of NBR1 homologs in different species. 147

Supplement 4: Heterologous protein production of HsNBR1-DsRED in $\triangle$ Smnbr1::Hsnbr1-

Dsred ${ }^{\text {ect }}$ strains 148

Supplement 5: Fluorescence localization of SmNBR1-EGFP in the complemented $\Delta$ Smnbr1 strain. 149

Supplement 6: Quantitative Western experiment of vacuolar degradation of EGFP-SmATG8 under amino-acid starvation condition in different autophagy mutants. 150

Supplement 7: Localization studies of SmRPL25-EGFP and SmRPS19-EGFP. 151

Supplement 8: Yeast-two hybrid assay of SmRPL25 and SmRPS19 with the cargo receptor SmNBR1. 152 


\section{List of abbreviations}

aa

3-AT

$\mathrm{AD}$

$A D E 2$

A. dest.

Atg

$a m p^{\mathrm{R}}$

$\mathrm{BD}$

$\mathrm{BiFC}$

BLAST

BMM

bp

$\mathrm{CC}$

cDNA

CFP

$\mathrm{cm}^{\mathrm{R}}$

Co-IP

CR

C-terminus

C-terminal

$\mathrm{Cvt}$

DAPI

DIC

DNA

dNTP

Dsred

DTT

ect

egfp

ER

$\mathrm{EtBr}$

$\mathrm{EtOH}$

final conc.

Gal4-AD

Gal4-BD

gDNA

HIS3

hph

hyg ${ }^{\mathrm{R}}$

JUBA

kan $^{\mathrm{R}}$

$\mathrm{kb}$

$\mathrm{kDa}$

LC3

LC/MS amino acids

3-amino-1,2,4-triazol

activation domain

enzyme involved in adenine synthesis

Aqua destillatum

autophagy-related gene

ampicillin resistance

binding domain

Bimolecular fluorescence complementation experiments

basic local alignment and search tool

biomalt maize medium

base pair

coiled coil

complementary DNA

cyan fluorescence protein

chloramphenicol resistance

Co-immunoprecipitation

conserved region

carboxy-terminus

carboxy-terminal

cytoplasm-to-vacuole targeting

4',6-Diamidino-2-Phenylindole, Dihydrochloride

differential interference contrast

deoxyribonucleic acid

2'-deoxy-nucleotids-5'triphosphate

encodes red fluorescence protein of Discosoma sp.

dithiothreitol

ectopical integrated

encodes enhanced green fluorescence protein of Aequorea victoria

endoplasmic reticulum

ethidium bromide

ethanol

final concentration

encodes the activation domain of the Gal4-transcription factor of Saccharomyces cerevisiae

encodes the binding domain of the Gal4-transcription factor of Saccharomyces cerevisiae

genomic DNA

enzyme for histidine biosynthesis

hygromycin B-phosphotransferase

hygromycin resistance

juxta UBA

kanamycin resistance

kilobase

kilo dalton

light chain 3

liquid chromatography-mass spectrometry 


\begin{tabular}{|c|c|}
\hline$L E U 2$ & enzyme involved in leucine biosynthesis \\
\hline LIR & LC3-interacting region \\
\hline nat & nourseothricin \\
\hline$n a t^{\mathrm{R}}$ & nourseothricin resistance \\
\hline NBR1 & neighbor of BRCA1 gene 1 \\
\hline $\mathrm{nt}$ & nucleotide \\
\hline N-terminus & amino-terminus \\
\hline N-terminal & amino-terminal \\
\hline OD & optical density \\
\hline ORF & open-reading frame \\
\hline $\mathrm{P}$ & phosphate \\
\hline $\mathrm{P}$ & promoter \\
\hline PAGE & polyacrylamide gel electrophoresis \\
\hline PAS & phagophore assembly site \\
\hline PB1 & phox and bem 1 \\
\hline PCR & polymerase chain reaction \\
\hline $\mathrm{PI} 3 \mathrm{~K}$ & phosphatidylinositol 3-kinase \\
\hline PtdIns & phosphatidylinositol \\
\hline $\mathrm{PE}$ & phosphatidylethanolamine \\
\hline PEG & polyethylene glycol \\
\hline RNA & ribonucleic acid \\
\hline $\mathrm{rpm}$ & revolutions per minute \\
\hline RT & room temperature \\
\hline SDS & sodium dodecyl sulphate \\
\hline ssi & single-spore isolate \\
\hline S.m. & Sordaria macrospora \\
\hline SQSTM1 & sequestosome 1 \\
\hline SWG & Sordaria Westergaards medium \\
\hline $\mathrm{T}$ & terminator \\
\hline TOR & target of rapamycin \\
\hline TRP1 & enzyme involved in tryptophan biosynthesis \\
\hline $\operatorname{trp} C^{\mathrm{T}}$ & terminator of the anthranilat synthase gene of Aspergillus nidulans \\
\hline $\mathrm{Ub}$ & ubiquitin \\
\hline UBA & ubiquitin association \\
\hline UBL & ubiquitin-like protein \\
\hline$U R A 3$ & enzyme involved in uracil biosynthesis \\
\hline UTR & untranslated region \\
\hline Vps & vacuolar protein sorting \\
\hline $\mathrm{Y} 2 \mathrm{H}$ & yeast-two hybrid \\
\hline YFP & yellow fluorescence protein \\
\hline wt & wild type \\
\hline $\mathrm{ZZ}$ & zinc-finger domain \\
\hline
\end{tabular}

Common used abbreviations and units of measurement are not enlisted. 


\section{Summary}

Autophagy is a conserved ubiquitous degradation process in eukaryotic cells which is mainly investigated in the baker's yeast Saccharomyces cerevisiae. It includes the random sequestration of defective and excessive proteins and organelles within a double-membraned autophagosome. In filamentous fungi, the main purposes of autophagy are the regulation of starvation adaptation and developmental processes. In yeast, 41 autophagy-related genes (atg) exist from which many are conserved in the homothallic ascomycete Sordaria macrospora $(\mathrm{Sm})$. One of the core autophagy genes is atg12 which is essential for autophagosome formation. Yeast-two hybrid analysis revealed a physical interaction of SmATG12 with both SmATG7 and SmATG3. A homokaryotic Smatg12 knockout strain could be generated which displayed reduced vegetative growth under nutrient-starvation conditions and was unable to form fruiting bodies. EGFP-labeled SmATG12 was detected in the cytoplasm and as punctate structures presumed to be phagophore structures. Furthermore, delivery of EGFP-labeled SmATG8 to the vacuole was entirely dependent on SmATG12.

In contrast to non-selective bulk autophagy, selective autophagy is characterized by cargo receptor proteins, which bind specific cargos such as organelles, damaged or harmful proteins or microbes for their autophagic degradation. The selective autophagy of protein aggregates and surplus organelles such as peroxisomes, mitochondria, ribosomes and nuclei is referred to as aggre-, pexo-, mito-, ribo-, and nucleophagy, respectively. By a GFP-Trap analysis followed by liquid chromatography mass spectrometry (LC/MS) using the core autophagy protein SmATG8 as bait SmNBR1 was identified, which is a putative homolog of the human autophagy cargo receptor neighbour of BRCA1 (NBR1). SmNBR1-DsRED co-localizes with EGFPSmATG8 at autophagosomes-like structures and in vacuoles. The interaction of both proteins was confirmed by means of yeast-two hybrid experiments, BiFC and Co-IP. Deletion of SmnbrI leads to impaired vegetative growth under starvation conditions and fruiting-body development that is characterized by a drastically reduced number of perithecia and mature ascospores. The phenotypically defects could be rescued by complementing the $\Delta$ Smnbr1 mutant with fulllength Smnbrl as well as partially with a human nbrl homolog. Additionally, Smnbrl is involved in pexophagy. A $\Delta$ Smnbrl mutant is neither able to use fatty acids as sole carbon source nor able to form fruiting bodies on $\mathrm{H}_{2} \mathrm{O}_{2}$-containing medium. The detection of numerous ribosomal subunit proteins in a GFP-Trap analysis using SmNBR1 as bait suggested that SmNBR1 is also involved in ribophagy. Quantitative Western-blot experiments and fluorescence microscopy revealed that degradation of SmRPL25-EGFP, a 60S ribosomal subunit protein, is impaired in $\Delta$ Smnbr1. Thus, it might be that SmNBR1 is the long-soughtafter ribophagy receptor for the large ribosomal subunit. 


\section{Zusammenfassung}

Autophagie ist ein Abbauweg, der in allen Eukaryonten konserviert ist. Um die Zelle auch unter Mangelbedingungen mit Nährstoffen versorgen zu können, werden in diesem Prozess nicht gebrauchte oder beschädigte Proteine und Organellen in den Vakuolen bzw. den Lysosomen abgebaut und der Zelle zur Verfügung gestellt. Der Autophagieprozess ist sehr gut in Saccharomyces cerevisiae analysiert und bislang konnten 41 (atg) Autophagiegene beschrieben werden von denen auch einige in dem filamentösen Ascomyceten Sordaria macrospora (Sm) identifiziert wurden. Eines der Hauptautophagiegene ist atg12, welches für die Autophagosomenbildung essentiell ist. In 'Yeast-two hybrid' Experimenten konnte eine physikalische Interaktion von SmATG12 mit SmATG7 und SmATG3 gezeigt werden. Zur funktionellen Charakterisierung des Smatg12 Gens wurde ein Deletionsstamm $\Delta \operatorname{Smatg} 12$ erstellt, welcher keine Perithezien bildet und somit steril ist. Der Phänotyp konnte durch Komplementierung bestätigt und verifiziert werden. Das Protein EGFP-SmATG12 konnte im Zytoplasma in Form von Punkten lokalisiert werden, welche voraussichtlich die 'Phagophore` darstellen.

Neben der nicht-selektiven Autophagie existieren auch selektive Autophagieprozesse, bei denen ein Cargo-Rezeptor ein spezifisches Organell, aggregierten Proteinkomplex oder Mikroben erfasst und selektiv abbaut. Der Abbau von Proteinaggregaten oder Organellen wie z. B. Peroxisomen, Mitochondrien, Ribosomen und Zellkernen wird jeweils als Aggre-, Pexo-, Mito-, Ribo-, und Nukleophagy beschrieben. In einer GFP-Trap und anschließender `liquid chromatography mass spectrometry` (LC/MS) Analyse konnte das Protein SmNBR1 als Interaktionspartner von SmATG8 identifiziert werden, welches als menschlicher Autophagie Cargo-Rezeptor neighbour of BRCA1 (NBR1) bekannt ist. SmNBR1-DsRED ko-lokalisiert mit EGFP-SmATG8 an Autophagosomen und in Vakuolen. Die Interaktion der beiden Proteine konnte durch Yeast-two hybrid, BiFC und Co-IP bestätigt werden. Eine Deletion von Smnbrl in S. macrospora wurde morphologisch untersucht und zeigt Defekte in der Fruchtkörperentwicklung wie auch das Wachstum unter Aminosäuremangel war stark reduziert. Der Phänotyp konnte durch Komplementierung des Smnbrl Genes bestätigt werden, was auch partiell durch das menschliche $n b r l$ Homolog gezeigt werden konnte. Außerdem ist SmNBR1 in Pexophagie involviert, da $\Delta$ Smnbr1 nicht in der Lage ist Fruchtkörper auf Medium mit langkettigen Fettsäuren als Kohlenstoffquelle sowie auf Medium mit $\mathrm{H}_{2} \mathrm{O}_{2}$ zu bilden. In GFP-Trap Analysen mit SmNBR1 als Köderprotein konnten viele ribosomale Proteine identifiziert werden. Quantitative Western-blot Experimente und Fluoreszenzmikroskopie zeigten, dass der Abbau von SmRPL25-EGFP, ein Protein der 60S ribosomalen Untereinheit, im $\triangle$ Smnbr1 verringert ist. SmNBR1 könnte daher der bislang noch nicht bekannte Rezeptor der Ribophagie sein. 


\section{Introduction}

\subsection{Overview of autophagy in eukaryotes}

Autophagy is an intracellular lysosomal (vacuolar) degradation process of cytoplasmic components (Cuervo 2004). It has a great variety of physiological and pathophysiological roles in eukaryotic cells and is conserved from yeast to man. The main purposes of autophagy are the regulation of starvation adaptation and developmental processes, clearance of old or defective intracellular proteins, organelles or invading microorganisms (Mizushima 2005; Wen and Klionsky 2016). In humans, defects in autophagy are involved in the etiology of numerous diseases, including cancer and neurodegenerative diseases (Yorimitsu and Klionsky 2005b; Milisav et al. 2015), whereas in fungi, defects in autophagy can cause impairment of multicellular development and reduced vegetative growth of fungal hyphae or defects in pathogenicity (Voigt and Pöggeler 2013a).

Although, autophagy is thought to be predominantly a cell-survival mechanism not only for restoring intracellular nutrient supply during starvation, it is also required for quality control to protect the cell against damages caused by toxic macromolecules and damaged organelles (Levine and Kroemer 2008; Mizushima et al. 2008).

Three distinct autophagy mechanisms have been described: macroautophagy, microautophagy, and chaperone-mediated autophagy (CMA) (Figure 1). Macroautophagy is the invagination of random or selected cytosolic contents by a double membrane of an autophagosome, whereas the direct uptake at the lysosomal or vacuolar membrane is termed microautophagy (Pollack et al. 2009; Li et al. 2012; Reggiori and Klionsky 2013). In micro- and macroautophagy, the cell content is degraded within the lysosome or the vacuole. In contrast to these two conserved pathways, CMA has been only found in mammals and does not rely on core autophagy-related (atg) genes (Okamoto 2014). The CMA is responsible for the degradation of specific cytosolic substrate proteins in cooperation with chaperones which bind target substrates. For this, proteins contain a targeting motif that is recognized by the cytosolic chaperone heat shock cognate protein of $70 \mathrm{kDa}$ (HSC70). Afterwards, substrate proteins bind to the lysosomal receptor lysosome-associated membrane protein type $2 \mathrm{~A}$ (LAMP-2A) and get translocated into the lysosomal lumen assisted by the chaperone. Once in the lysosomal matrix, substrate proteins are rapidly degraded by lysosomal proteases (Massey et al. 2004). 
A Macroautophagy
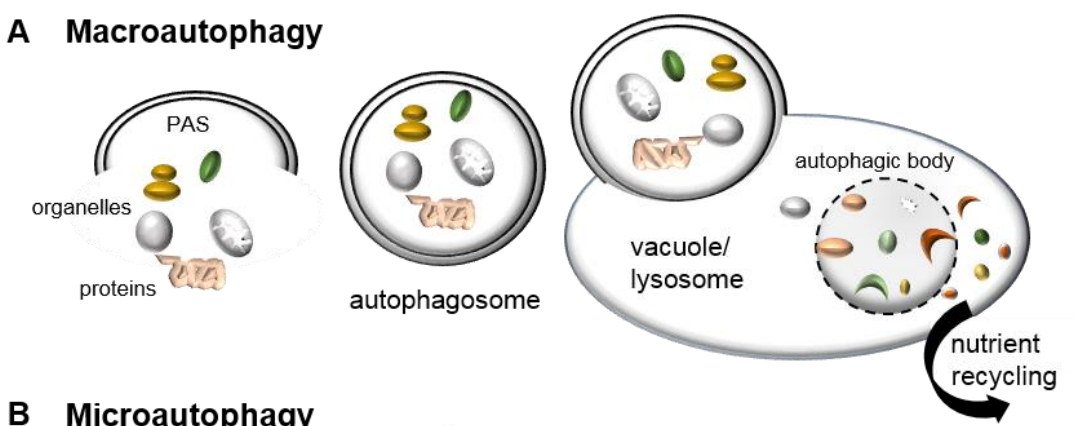

B Microautophagy
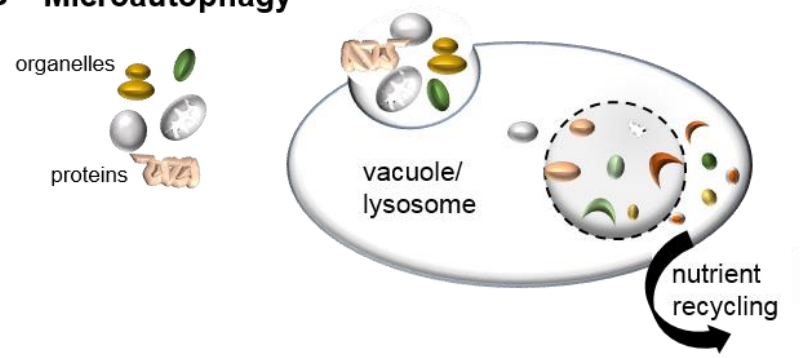

C Chaperone-mediated autophagy (CMA)

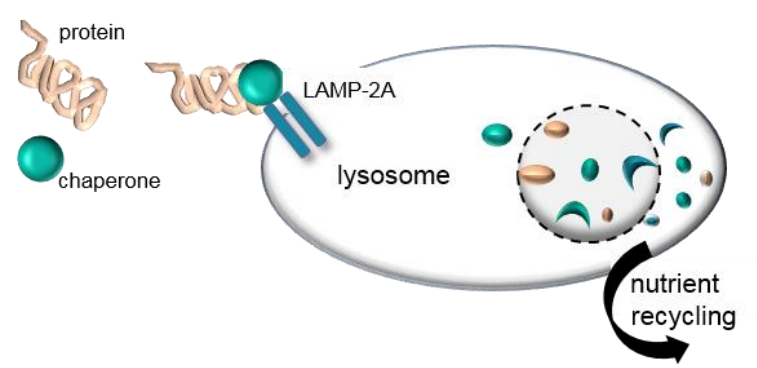

Figure 1: Overview of autophagy processes.

(A) Macroautophagy begins with the formation of a double membrane to form the PAS (phagophore assembly site). The membrane elongates to sequester cellular elements, including organelles and proteins, within an autophagosome. The autophagosome moves to a vacuole/lysosome for fusion with its outer membrane. The autophagic body will be degraded by hydrolytic enzymes in the vacuole/lysosome for nutrient supply. (B) In microautophagy, an invagination of the vacuolar/lysosomal membrane allows the direct uptake of cytosolic components such as organelles and proteins for degradation by hydrolases. (C) CMA is specific for the removal of cytosolic proteins with a pentapeptide motif recognized by the chaperone protein HSC70. Binding of this complex to the lysosomal LAMP-2A receptor leads to protein internalization and degradation in the lysosome.

Macroautophagy is the best studied and characterized process, and therefore it is often termed “autophagy”. Most of our current knowledge on autophagy was provided by a series of genetic screens in the yeast Saccharomyces cerevisiae, which was established as a good model organism to explain the autophagy mechanism (Suzuki and Ohsumi 2007).

\subsection{Macroautophagy in the yeast S. cerevisiae}

Since the 1990s macroautophagy of the baker's yeast $S$. cerevisiae represents the best-studied autophagy system (Cao et al. 2008). Mutant screenings in different yeast species like S. cerevisiae, Pichia pastoris and Hansenula polymorpha identified 41 so called atg genes, of which 19 are required for all autophagy-related pathways while the others are involved in the 
induction of specific autophagy processes in response to different physiological conditions, and in selective autophagy (Ohsumi 2001; Araki et al. 2013; Nazarko 2014; Mochida et al. 2015; Noda and Inagaki 2015; Yao et al. 2015). Beside the non-selective bulk autophagy, selective autophagy is characterized by cargo receptor proteins, which are able to bind a specific cargo such as organelles, proteins or microbes for their autophagic degradation (Kraft et al. 2010; Stolz et al. 2014; Zaffagnini and Martens 2016).

Macroautophagy can be distinguished in the consecutive steps of induction, nucleation, expansion and vesicle closure, fusion with the vacuole and breakdown of the cargo (Rubinsztein et al. 2012) (Figure 2). The induction of the process promotes the de novo synthesis of the autophagosomal double membrane and occurs in S. cerevisiae under nutrient starvation conditions such as starvation for carbon, nitrogen or essential components like amino- and nucleic acids or by rapamycin treatment (Noda et al. 2002).
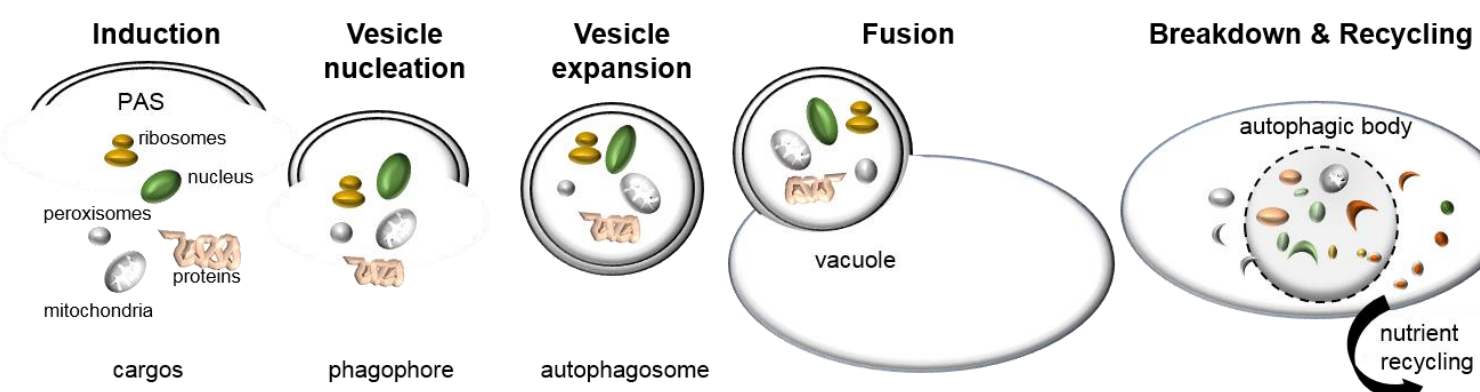

Figure 2: Five sequential steps of autophagy in $S$. cerevisiae.

After induction of autophagy, an initial sequestering phagophore is assembled at the PAS. Expansion and curvature of the phagophore leads to the engulfment of the cargo (cytoplasm, proteins and organelles) into the doublemembraned autophagosome. Fusion of the autophagosomal outer membrane with the vacuolar membrane results in the release of the autophagic body which is surrounded by the inner autophagosomal membrane. Autophagic bodies and the sequestered cargos are digested by hydrolytic enzymes. Finally, the breakdown products are exported into the cytoplasm for reuse. PAS, phagophore assembly site.

Under nutrient-rich conditions the core autophagic protein Atg13 is highly phosphorylated by the target of rapamycin (TOR) kinase (Figure 3). Starvation conditions lead to the inactivation of the TOR kinase and its inability to phosphorylate Atg13. In its unphosphorylated state, Atg13 forms together with the kinase Atg1, Atg11/17, Atg29 and Atg31 the Atg1-kinase complex. This complex is capable of phosphorylating Atg2 and the transmembrane protein Atg9 (Papinski et al. 2014; Papinski and Kraft 2016) (Figure 3A). The proteins Atg1, Atg13, Atg11/17, Atg29 and Atg31 are localized at the phagophore assembly site (PAS) (Suzuki et al. 2001). During selective autophagy Atg17 of the Atg1-kinase complex is exchanged by the scaffolding protein Atg11 (Yorimitsu and Klionsky 2005a). 
The nucleation of the autophagosomal membrane requires the phosphorylation of phosphatidylinositol (PtdIns) by complex I consisting of vacuolar protein sorting (Vps)34, Vps15, Atg14, Atg6 and Atg38. Subsequently, phosphorylated Atg2 and Atg9 can then localize at the PAS. Atg2 functions as a connector between Atg9 and the phosphatidylinositol-3phosphate (PtdIns(3)P) binding protein Atg18 and thereby Atg9 delivers membrane structures to the expanding PAS (Figure 3B) (Reggiori et al. 2005; Araki et al. 2013; Reggiori and Klionsky 2013; Papinski et al. 2014). Recently, the small protein Atg41 was identified, which is suggested to be involved in the autophagosome biogenesis, through interaction with Atg9 (Yao et al. 2015).

During expansion, the Atg12 and Atg8 conjugation systems are necessary to build the double membrane of the autophagosome. Both are ubiquitin ( $\mathrm{Ub}$ )-like proteins and become conjugated in a Ub-like manner. Atg8 and Atg12 do not share any primary sequence homology with Ub, nevertheless, their crystal structures reveal similarities (Sugawara et al. 2004; Suzuki et al. 2005). Atg8 is initially processed to a glycine-exposed form by the protease Atg4, then activated in an ATP-dependent manner by the E1-like enzyme Atg7, subsequently transferred to the E2like enzyme Atg3, and finally conjugated to the amino group of phosphatidylethanolamine (PE) (Mizushima et al. 1998; Shintani et al. 1999; Tanida et al. 1999; Ichimura et al. 2000; Ohsumi 2001). Atg12 is also activated by the E1-like enzyme Atg7 but is transferred to the E2-like conjugating enzyme Atg10, which covalently attaches it to a lysine residue of Atg5 (Mizushima et al. 1998; Shintani et al. 1999). The resulting Atg12 Atg5 conjugate then forms a complex with the coiled-coil protein Atg16, and this complex acts as a ubiquitin E3-like ligase for the Atg8-PE conjugation reaction by stimulating the activity of $\operatorname{Atg} 3$ and promoting the transfer of Atg8 from Atg3 to the PE substrate (Mizushima et al. 1999; Hanada et al. 2007; Fujita et al. 2008). Both Ub-like conjugates (Atg8-PE and the Atg12 Atg5-Atg16 complex) are localized to the PAS (Suzuki et al. 2001) (Figure 3C). In S. cerevisiae, Atg8-PE recruits the Atg12 Atg5Atg16 complex for membrane localization by recognizing a non-canonical Atg8-interacting motif (AIM) in Atg12 (Kaufmann et al. 2014). In the growing phagophore, the Atg12 Atg5Atg16 complex localizes exclusively to the convex side of the growing cup-shaped phagophores, while Atg8-PE localizes at both sides of the phagophore (Mizushima et al. 2003; Suzuki et al. 2013). 
A Induction

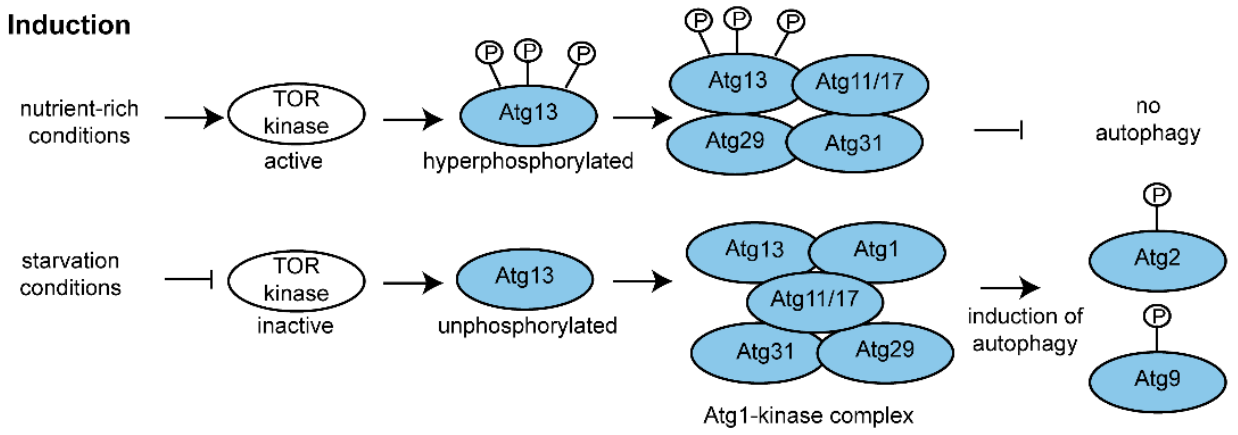

B Vesicle nucleation
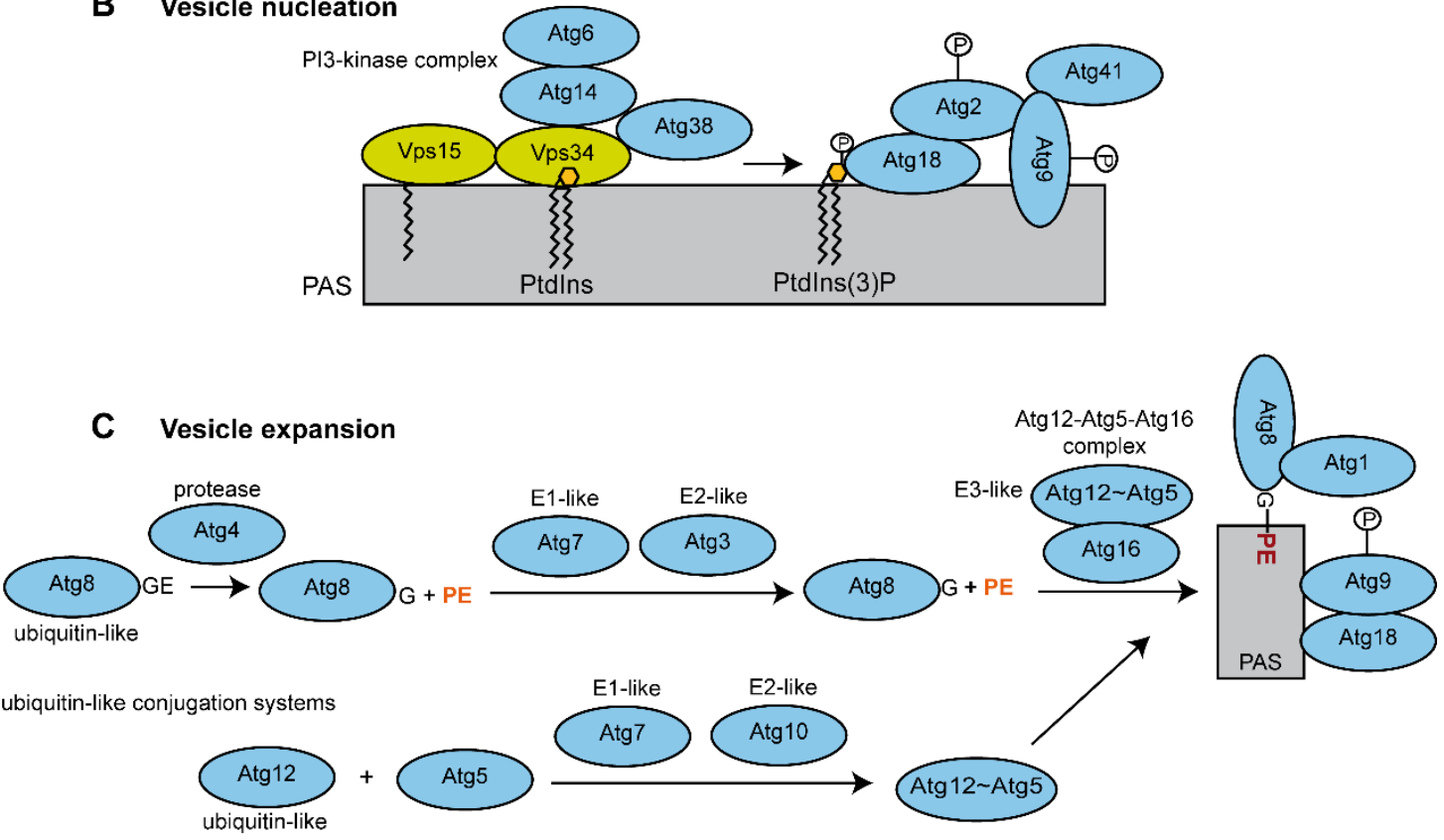

D Fusion Breakdown \& Recycling

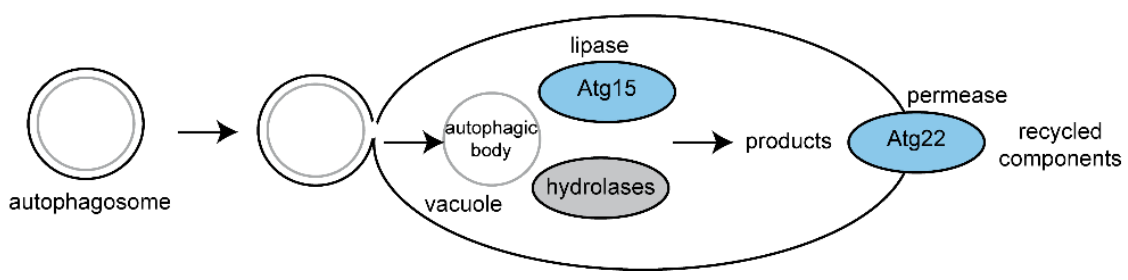

Figure 3: Proteins involved in autophagy in the yeast $S$. cerevisiae.

(A) Induction of autophagy under nutrient-starvation conditions followed by the formation of the Atg1-kinase complex. (B) Vesicle nucleation mediated by the phosphorylation of phosphatidylinositol at the PAS. (C) Expansion of the phagophore by conjugation of Atg8-PE to the PAS via the Atg8 and Atg12 conjugation pathways. (D) Fusion of the outer membrane of the autophagosome with the vacuolar membrane releasing the autophagic body and breakdown within the vacuole by hydrolases. TOR, target of rapamycin; PAS, phagophore assembly site; PE, phosphatidylethanolamine; PtdIns, phosphatidylinositol; PtdIns(3)P, phosphatidylinositol-3-phosphate; PI3, phosphoinositide 3; P, phosphorylation.

On the convex membrane site of the phagophores, Atg16 promotes oligomerization of the Atg12 Atg5-Atg16 complex and assembles with Atg8-PE into a two-dimensional meshwork (Kaufmann et al. 2014). Cytosol and organelles are then surrounded by this membrane forming the autophagosome, which is transported to the vacuole. The outer membrane of the 
autophagosome is able to fuse with the vacuolar membrane and releases a single-membraned autophagic body into the lumen of the vacuole. The vacuolar lipase Atg15 degrades the inner autophagosomal membrane and the content of the autophagic body is digested by hydrolytic vacuolar enzymes (Epple et al. 2001) (Figure 3D). The permease Atg22 is an integral vacuolar membrane protein and contributes to the release of vacuolar degradation products into the cytoplasm (Yang et al. 2006). The recycled components can be reused for anabolic processes in the cell.

\subsection{Selective autophagy processes mediated by cargo receptors}

In addition to non-selective autophagy, the degradation of cytoplasm and organelles in a random manner, selective autophagy processes deliver specific cargos to the lysosome/vacuole and can be subdivided into mitophagy (for mitochondria), pexophagy (for peroxisomes), ribophagy (for ribosomes), reticulophagy (for the endoplasmic reticulum, ER), nucleophagy (for the nucleus) and aggrephagy (for aggregates) and the cytoplasm-to-vacuole targeting (Cvt)-pathway exclusively present in S. cerevisiae (Khalfan and Klionsky 2002; Kim et al. 2007; Meijer et al. 2007b; Kraft et al. 2008; He and Klionsky 2009; Kanki and Klionsky 2009; Kraft et al. 2010; Lynch-Day and Klionsky 2010; Mochida et al. 2015; Nakatogawa and Mochida 2015) (Figure 4). The Cvt pathway mediates the transport of hydrolytic enzymes such as amino peptidase $\underline{1}$ (Ape1) and $\underline{\alpha-m}$ mannosidase (Ams1) from the cytoplasm under non-starvation conditions.

The detailed mechanisms of these selective pathways remain to be characterized but some common principles are arising (Figure 4). It is known that a receptor recognizes and binds a specific cargo to direct it to the autophagosomes. During this process the core autophagy machinery is required for all the selective types of autophagy. Often, ubiquitin acts as a signaling molecule for selective autophagy (Kraft et al. 2010).

In yeast but not in filamentous fungi, during nutrient-rich conditions, the Cvt pathway uses the receptor Atg19 and the related protein Atg34 for the selective transport of hydrolytic enzymes from the cytosol to the vacuole (Figure 4B). Atg19 binds directly to its cargo Ape1 or Ams1, whereas Atg34 binds only Ams1, to form the so called Cvt complex (Watanabe et al. 2010). Subsequently, the receptors mediate the recruitment of the Cvt complex to the PAS via interaction with Atg11 (Zaffagnini and Martens 2016). The cargo receptors Atg19 and Atg34 are able to bind to Atg8 via a conserved LC3-interacting region (LIR), also known as AIM, to mediate the engulfment of the complex by vesicle formation (Pankiv et al. 2007; Ichimura et al. 2008). The LIR motif allows the interaction with LC3-family proteins including the 
mammalian homolog of the yeast Atg8 protein and consists of the consensus sequence $\mathrm{X}_{-3}-\mathrm{X}_{-2^{-}}$ $\mathrm{X}_{-1}-\left(\mathrm{W} / \mathrm{Y} / \mathrm{F}_{0}\right)-\mathrm{X}_{1}-\mathrm{X}_{2}-\left(\mathrm{L} / \mathrm{I} / \mathrm{V}_{3}\right)-\mathrm{X}_{4}-\mathrm{X}_{5}$ (Alemu et al. 2012; Suzuki et al. 2014). The scaffold protein Atg11 is required for the localization of the Cvt vesicle to the PAS (Khalfan and Klionsky 2002; Shintani et al. 2002; Yorimitsu and Klionsky 2005a; Watanabe et al. 2010; Sawa-Makarska et al. 2014). Phosphorylation of the cargo receptors promote their interaction with Atg11. The kinase Hrr25 (프 and radiation repair) was identified to phosphorylate Atg19 whereas an unknown kinase exists for Atg34 (Pfaffenwimmer et al. 2014; Tanaka et al. 2014). Mitophagy represents another selective autophagy process in S. cerevisiae and higher eukaryotes, which is important for the cell to remove damaged or harmful mitochondria. In yeast, a protein of the outer mitochondrial membrane, $\operatorname{Atg} 32$, was identified as a receptor for mitophagy (Kanki and Klionsky 2009; Okamoto et al. 2009). After phosphorylation by the casein kinase 2 (CK2) Atg32 is able to interact with Atg11 and Atg8. Interaction of Atg32 and Atg8 is mediated by the LIR motif (Farre et al. 2013; Kanki et al. 2013; MacVicar 2013) (Figure 4C).

The selective degradation of peroxisomes via autophagy uses Atg30 in P. pastoris or Atg36 in S. cerevisiae as cargo receptors. Both receptors are phosphorylated by kinases and interact with peroxisomal membrane proteins Pex3 and/or Pex14 (Farre et al. 2008; Farre et al. 2013; Tanaka et al. 2014) (Figure 4C). Upon phosphorylation they interact with core autophagy machinery components Atg11 and Atg17 (Farre et al. 2013; Tanaka et al. 2014). The cargo receptors Atg30 and Atg36 bind directly to the Atg8 protein in a LIR-dependent manner (Farre et al. 2008; Motley et al. 2012). Recently, the acyl-CoA binding protein Atg37 was identified as a new component of the pexophagy process in P.pastoris. Atg37 is an integral peroxisomal membrane protein that binds Pex3 and Atg30, but not Pex 14 or Atg8. The role of Atg37 is to facilitate the Atg30-Atg11 interaction and therefore to regulate the dynamic of this process (Nazarko et al. 2014; Burnett et al. 2015).

Aggrephagy uses the molecular tag ubiquitin for the identification of a protein aggregate for autophagic degradation (Figure 4D). 

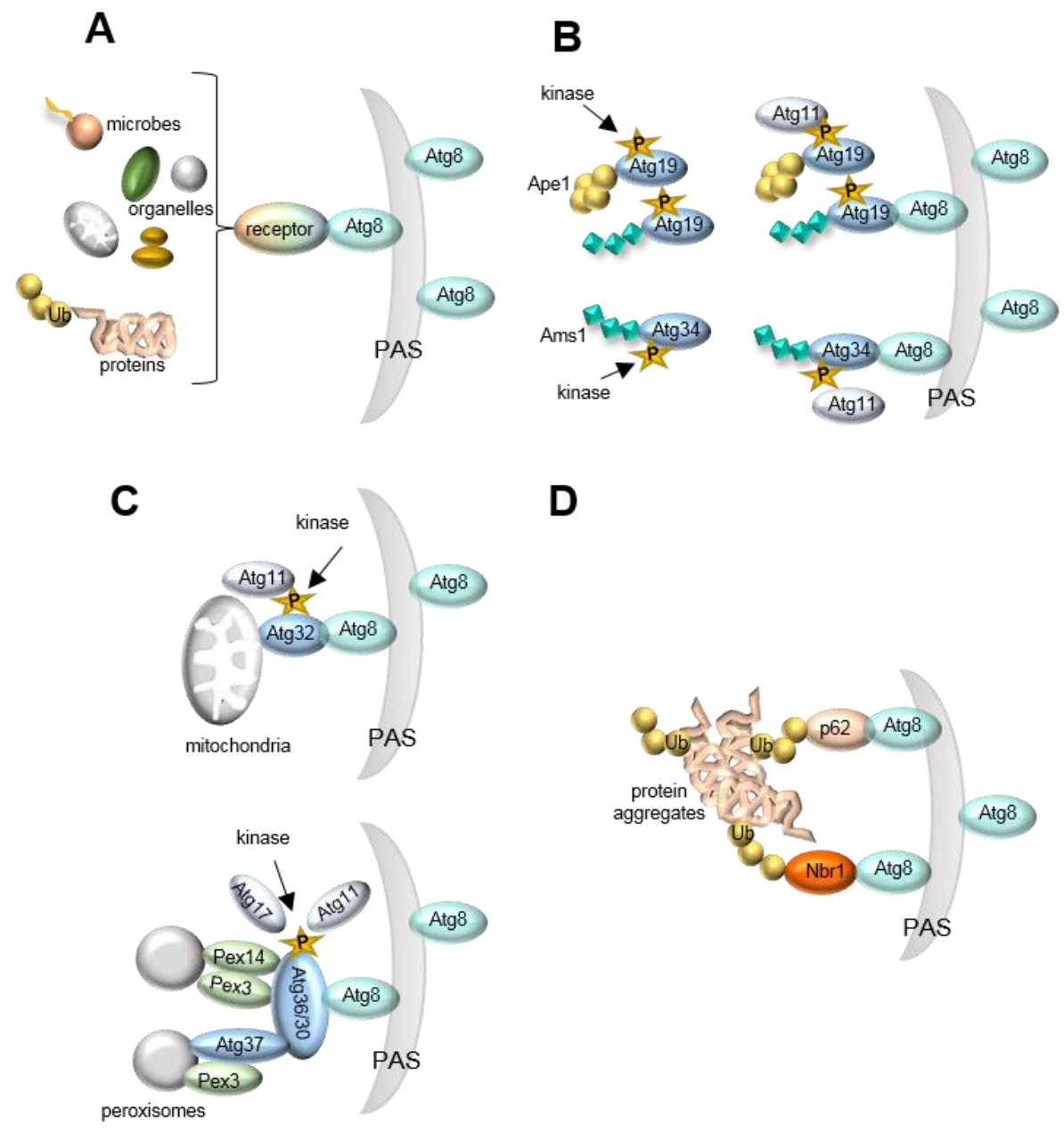

D

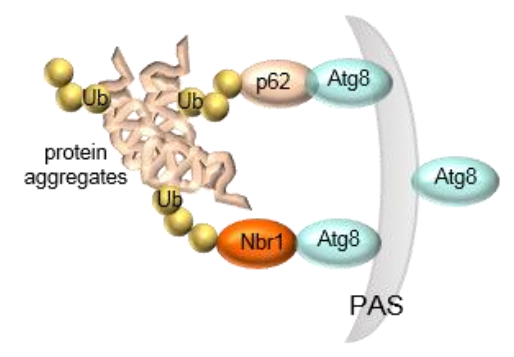

Figure 4: Cargo recognition during selective autophagy.

(A) General scheme of cargo recognition during selective autophagy. A receptor is able to bind the substrate (organelles, proteins, microbes) in some cases labeled with ubiquitin (Ub) and interacts with Atg8 family proteins of the PAS (Johansen and Lamark 2011). (B) In S. cerevisiae, the Cvt pathway transports the hydrolytic enzymes Ape1 and Ams1 to the vacuole. At the PAS, the cargo receptors Atg19 and Atg34 are phosphorylated. Phosphorylated Atg19 binds Ape1 or Ams1 and phosphorylated Atg34 binds Ams1. Subsequently, both receptors can interact with Atg11 and Atg8 (Zaffagnini and Martens 2016). (C) Organelle specific autophagy processes in yeast. During mitophagy, Atg11 connects the selective receptor Atg32, a mitochondrial outer membrane protein, to Atg8 at the PAS. During this process Atg32 gets phosphorylated (MacVicar 2013). The selective autophagy degradation of peroxisomes uses Atg30 in P. pastoris and Atg36 in S. cerevisiae, which are localized at the peroxisomal membrane, and interact with Pex3 and/or Pex14. The cargo receptors are phosphorylated and upon induction they interact with core autophagy machinery components Atg11 and Atg17 (Farre et al. 2008; Motley et al. 2012). The cargo receptors bind directly to the Atg8 protein which is localized to the PAS. Additionally, Atg37 is an integral peroxisomal membrane protein which can bind Pex3 and Atg30 to facilitate the Atg30/36-Atg11 interaction (Nazarko 2014). (D) In mammals, ubiquitinated proteins bind to the cargo receptors p62 and/or Nbr1. Both receptors are connected to Atg8 family proteins in the autophagosomal membrane. This process is called aggrephagy (Stolz et al. 2014). PAS, phagophore assembly site; P, phosphorylation.

It provides an alternative to the proteasomal degradation pathway and allows the degradation of toxic, aggregated or long-living proteins (Kraft et al. 2010). In mammals but not in yeast, NBR1 (neighbour of BRCA1) and p62/SQSTM1 (sequestosome 1) are described as cargo receptors for the degradation of ubiquitinated substrates. Both can interact with ATG8 via the 
LIR motif, which is an essential structural component of the autophagosomal membrane, and with ubiquitin via a ubiquitin-asssociated (UBA) domain (Pankiv et al. 2007; Kirkin et al. 2009; Stolz et al. 2014).

Despite the fact that mammalian proteins p62 and NBR1 have a similar domain organization, not all domains present in NBR1 are present in p62 (Deosaran et al. 2013). In eukaryotes, NBR1 is highly conserved while p62 is only found in metazoans. Homologs of NBR1 have been described in plants and mammals, but not in S. cerevisiae. In plants, the homolog of NBR1 has been shown to interact with the homolog of ATG8 and is involved in the selective autophagy degradation of ubiquitinated proteins (Svenning et al. 2011; Zhou et al. 2013). The mammalian NBR1 protein is characterized by an N-terminal Phox and Bem1 (PB1) domain responsible for heterodimerization with p62, followed by two coiled-coil (CC) domains for homo-dimerization,

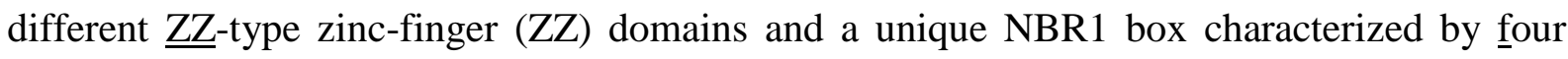
tryptophan $(\underline{\mathrm{W}})(\mathrm{FW})$ residues with unknown function. A C-terminal UBA domain is capable of binding ubiquitinated proteins (Hofmann and Bucher 1996). In close proximity to the UBA domain a 22 amino acid Juxta UBA (J) domain was identified in metazoan NBR1 homologs. This domain forms a membrane-interacting amphipathic $\alpha$-helix (Mardakheh et al. 2010). The NBR1, CC and J domains are lacking in the p62 protein (Kirkin et al. 2009; Kraft et al. 2010; Svenning et al. 2011) (Figure 5).

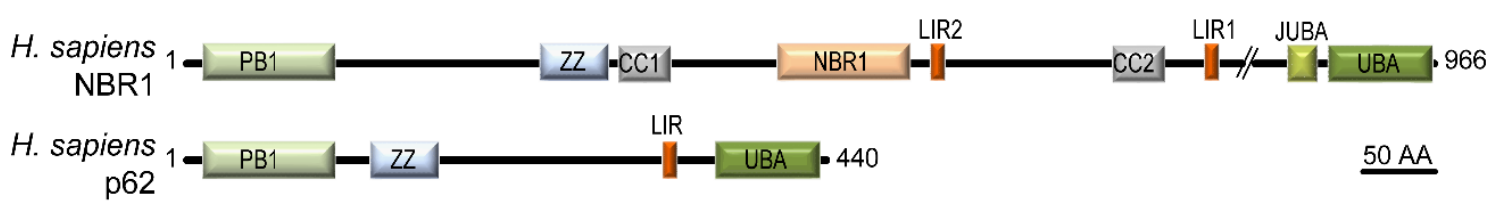

Figure 5: Domain architecture of the Homo sapiens NBR1 and p62.

The NBR1 protein and its counterpart p62 are characterized by common domains like PB1, Phox and Bem1 domain; ZZ, ZZ-type zinc-finger domain; LIR, LC3-interacting region; UBA, ubiquitin-associated domain. NBR1 contains additionally CC, coiled-coil domain; the typical NBR1-domain and N-terminal of the UBA domain in close proximity the JUBA, juxta-ubiquitin-associated domain. Functional domains are represented as colored boxes, with similar colors indicated similar domains. Amino-acid scale bar is indicated.

In $S$. cerevisiae, the Cvt receptor Atg19 is suggested to be a functional equivalent to NBR1 (Kraft et al. 2010). Fission yeast Schizosaccharomyces pompe has neither the Cvt pathway nor its receptor Atg19 but expresses a NBR1-like protein called Nbr1. This protein functions as a receptor for delivering the hydrolytic enzymes Ape2 and Lap2 (lamina-associated polypeptide 2) to the vacuole via an unconventional autophagy-independent mechanism (Liu et al. 2015; Mizushima 2015). Interestingly, the homolog in fission yeast lacks the PB1 domain, the LIR motif, CC and UBA domains but it possesses a similar NBR1 box. 
With the exception of zygomycetes, NBR1 homologs of all fungi lack the UBA domain (Kraft et al. 2010). So far, the function of NBR1 homologs in filamentous fungi is unknown.

In contrast to this, it is known that in mammals, beside its function in aggrephagy, NBR1 is involved in pexophagy (Deosaran et al. 2013).

\subsection{Pexophagy, the autophagic degradation of peroxisomes}

Peroxisomes are abundant single membrane-bound organelles in eukaryotic cells and have various physiological functions based on their role in diverse metabolic pathways. The primary role of fungal peroxisomes is the $\beta$-oxidation of fatty acids and detoxification of reactive oxygen species (ROS). Fungal mutants lacking peroxisomes fail to grow on media containing fatty acids as sole carbon source (Erdmann et al. 1989; Hynes et al. 2008). In filamentous fungi, peroxisomes are also responsible for the biosynthesis of biotin, the formation of secondary metabolites including the biosynthesis of penicillins, polyketides, terpenes and paxilline (Maggio-Hall et al. 2005; Saikia and Scott 2009; Imazaki et al. 2010; Bartoszewska et al. 2011; Tanabe et al. 2011). Moreover, peroxisomes are involved in plant pathogenicity of fungi (Kimura et al. 2001; Asakura et al. 2006), sexual development and growth (Bonnet et al. 2006; Managadze et al. 2007; Peraza-Reyes et al. 2008). The biogenesis and proteostasis of peroxisomes is essential to adapt to environmental changes and to control metabolic events in the cell. To maintain a healthy cellular peroxisome population, dysfunctional or useless peroxisomes are degraded by pexophagy. This turnover process may occur randomly (bulk sequestration of cytoplasm portions) or selectively. In filamentous ascomycetes, peroxisomes arise predominantly at the hyphal tips and their biogenesis is controlled by pex genes, which encode proteins called peroxins (Distel et al. 1996; Kiel et al. 2006). Over 30 peroxins have been identified in fungal genomes. Most of them are evolutionarily conserved but some are specific for filamentous fungi (Kiel et al. 2006; Managadze et al. 2007; Peraza-Reyes et al. 2011; Li et al. 2016). Peroxins are involved in the de novo formation of peroxisomes from the endoplasmatic reticulum, in their division and the import of folded proteins into the peroxisomal matrix. Two major classes of peroxisomal targeting signals (PTS) are present in peroxisomal matrix proteins. The PTS1 sequence comprises three C-terminal amino acids characterized by the amino-acid sequence S/A-R/K-L. Other peroxisomal matrix proteins have a PTS2 sequence close to the N-terminus with the consensus sequence R-L-X5-H-L (where X represents any amino acid) (Gould et al. 1989; Swinkels et al. 1991; Petriv et al. 2004; Brocard and Hartig 2006). In S. cerevisiae, two types of cytosolic receptor proteins have been identified for the 
import of the two classes of matrix proteins. Each recognizes one of the PTSs, PEX5 and PEX9 PTS1-matrix proteins and the PTS2 receptor complex PEX7 together with its co-receptors PEX18 and PEX21 PTS2 matrix proteins (Kiel et al. 2006; Kiel and van der Klei 2009; Effelsberg et al. 2016). The cargo-receptor complex is then transported to an import pore present in the peroxisomal membrane. After docking, the translocation of the cargo-receptor complex across the peroxisomal membrane occurs through distinct PTS1 and PTS2 pores (Montilla-Martinez et al. 2015). The pore for PTS1 proteins consists of the PTS1 receptor PEX5 and the protein PEX14 or PEX13, whereas the PTS2 pore is characterized by the three peroxins PEX14, PEX17 and PEX18 (Managadze et al. 2007; Opalinski et al. 2010). The translocation is followed by the dissociation of the cargo receptor and recycling of the receptor to the cytosol for further import cycles (Erdmann and Schliebs 2005).

The key point in pexophagy, as in other selective autophagy pathways, is the mechanism by which the core autophagy machinery is directed to degrade primarily peroxisomes. Until now, four pexophagy receptors have been identified: Atg30 in P. pastoris and related yeasts (Farre et al. 2008; Nazarko 2014), Atg36 in S. cerevisiae and related yeasts (Motley et al. 2012) and NBR1 and/or p62 for mammalian cells (Kim et al. 2008; Deosaran et al. 2013). The receptors display a modular composition of binding domains and motifs for cargo delivery to the site of developing and engulfing autophagosomes. Common for the most of these receptors is the presence of a LIR motif and a UBA domain (Behrends and Fulda 2012).

In mammals, pexophagy can be induced by expression of ubiquitinated peroxisomal membrane proteins and it has been demonstrated that p62 together with LC3-II (ATG8) is required for the degradation of ubiquitin-decorated peroxisomes by interacting with an unknown peroxisomal protein (Kim et al. 2008). However, Deosaran et al. (2013) showed that pexophagy is also induced by overexpression of NBR1, which promotes peroxisome clustering around NBR1 oligomers and autophagic peroxisome degradation. The $\mathrm{J}$ and the UBA domains of mammalian NBR1 are required for pexophagy. Moreover, ubiquitinated PEX5 and overexpression of PEX3 target NBR1 to clustered peroxisomes (Deosaran et al. 2013; Yamashita et al. 2014).

In P. pastoris, the acyl-CoA binding protein Atg37 is an integral peroxisomal membrane protein that binds, similar to the receptor Atg30, Pex3 in the peroxisomal membrane (Nazarko et al. 2014; Burnett et al. 2015). Atg30 can additionally bind the peroxisomal membrane protein Pex14 (Farre et al. 2008). Further, Atg37 interacts with Atg30 to facilitate the engagement of the scaffold protein Atg11 (Nazarko et al. 2014). To bind both Atg11 and the phagophore protein Atg8, Atg30 has to be phosphorylated by a yet unknown kinase (Farre et al. 2008; Nazarko et al. 2014) (Figure 4C). The S. cerevisiae Atg36 receptor is recruited to peroxisomes 
also by Pex3. It is phosphorylated by the kinase Hrr25 and interacts with the scaffold protein Atg11 (Tanaka et al. 2014). Atg36 remains to be associated with Atg8 for autophagosomes formation and like other receptors for selective autophagy it is broken down together with its substrate in the vacuole (Motley et al. 2012).

So far, it is unknown how pexophagy is regulated in filamentous fungi. In addition to peroxisomes some filamentous fungi possess a peroxisomal organelle called Woronin body, which is responsible to seal damaged hyphae by plugging the septal pore (Markham 1994). Woronin bodies originate from peroxisomes (Jedd and Chua 2000).

Filamentous fungi grow via elongation of the hyphal tip and form hyphae in which many cellular compartments are interconnected through septal pores that allow the passage of cytoplasm and organelles between neighboring septa (Markham 1994; Lew 2005; Ng et al. 2009). This mode of cellular organization is a benefit and risk because the cell is able to respond to environmental changes and to undergo developmental processes but in case of hyphal wounding cytoplasmic loss takes place. When hyphae lyse, their septal pores need to be sealed to prevent complete cytoplasm leakage and cell death. This function is exclusively undertaken by an organelle known as Woronin body (Woronin 1864) and is uniquely present in Pezizomycotina (Maruyama et al. 2005; Jedd 2011). The main component of Woronin bodies is the protein HEX1, firstly identified in Neurospora crassa (Jedd and Chua 2000). The $N$. crassa HEX1 protein, with a predicted molecular mass of $19.13 \mathrm{kDa}$, has the PTS1 SRL motif as a peroxisomal targeting signal (Jedd and Chua 2000). Upon import of HEX1 into peroxisomes self-assembly of HEX1 takes place and Woronin bodies proliferated by growth and division. The Woronin-body sorting complex (WSC) recruits HEX1 to the matrix face of the peroxisomal membrane (Liu et al. 2008) and promotes the budding from peroxisomes which involves the function of PEX11. A specific protein leashin (LAH) tethers the Woronin body to septa (Ng et al. 2009). Deletion of the hexl gene in N. crassa results in the disappearance of Woronin bodies and is associated with severe cytoplasmic bleeding upon hyphal lysis (Jedd and Chua 2000). Degradation of Woronin bodies by autophagy has not yet been analyzed in filamentous fungi.

Beside peroxisomes, another organelle that has been described to be selectively degraded by autophagy are ribosomes. 


\subsection{Ribophagy, the autophagic degradation of ribosomes}

All cellular proteins are synthesized by ribosomes, whose biogenesis is a complex multi-step and the most energy consuming cellular process. The biogenesis and degradation of ribosomes is therefore tightly controlled upon nutrient limitations (Warner 1999). A study has shown that the selective autophagy of surplus ribosomes balances the ribosome biogenesis (Kraft et al. 2008). In S. cerevisiae, it has been demonstrated that ribosomes are regulated in their number by ribophagy. Under nutrient-starvation conditions ribosomal proteins are degraded faster than other cytoplasmic proteins. It was demonstrated that the transport of ribosomes to the vacuole relies on core autophagy components such as Atg1 and Atg7. A mutant screening in S. cerevisiae focusing on defects in ribosome turnover upon starvation, identified the ubiquitinspecific processing protease 3 (Ubp3) and its cofactor brefeldin A sensitivity 5 (Bre5). Together with the cell division cycle 48 (Cdc48) AAA-ATPase and the ubiquitin binding protein ubiquitin fusion degradation 3 (Ufd3) they are required for the selective degradation of the large 60S ribosomal subunit, but not for the small 40S subunit (Kraft et al. 2008; Ossareh-Nazari et al. 2010). When Ubp3 is asscociated with its cofactor Bre5 it removes ubiquitin from damaged or misfolded proteins (Brew and Huffaker 2002) including the large ribosomal protein Rpl25. This deubiquitination of Rpl25 is required to trigger the uptake of the large ribosomal subunit by the autophagosome, followed by degradation of ribosomal proteins in the vacuole. Under nutrient-rich conditions Rp125 is ubiquitinated by the E3 ligase Ltn1 (listerin E3 ubiquitin protein ligase 1). Ltn1 targets the lysine 74 of the Rpl25 and thereby protecting the $60 \mathrm{~S}$ ribosomal subunit against ribophagy in a Ubp3-antagonistic manner (Ossareh-Nazari et al. 2014) (Figure 6).

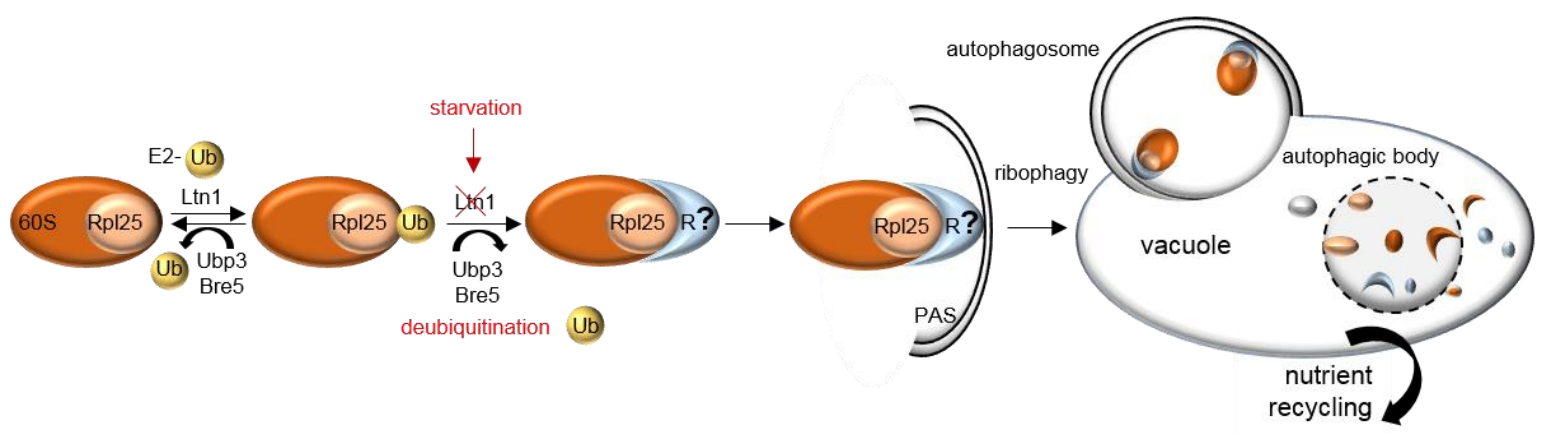

Figure 6: Model for the role of Ltn1 and Ubp3/Bre5 in starvation-induced ribophagy in S. cerevisiae (modified after Ossareh-Nazari et al. (2014)).

Under nutrient-rich conditions Ltn1 ubiquitinates Rp125 from the large ribosomal subunit. Under starvation conditions the level of Ltn1 decreases and the Ubp3/Bre5 complex acts in the deubiquitination of Rpl25, which is then targeted by a still unknown autophagy cargo receptor. Subsequently, the large ribosomal subunit is transported to the vacuole and the proteins are degraded by hydrolytic enzymes. 60S, 60S ribosomal subunit; R describes an unidentified cargo receptor; PAS, phagophore assembly site; Ub, ubiquitin. 
Under starvation conditions the levels of Ltn1 decrease and the Ubp3/Bre5 complex acts in the deubiquitination of Rpl25, which is then targeted to selective autophagy by a still unknown receptor (Figure 6). Recently it was reported that ribophagy requires in addition the autophagy scaffold protein Atg11 and is upregulated by the protein kinase Rim15 (Waliullah et al. 2016). The selectivity "receptor" for ribosome-autophagosome assembly is unknown and it remains to be investigated in more detail how ribosomes are subjected to autophagy or how they are recognized by the receptor and how ribophagy is regulated in eukaryotes.

\subsection{Sordaria macrospora: a model organism to study fruiting-body development}

The ascomycete Sordaria macrospora, which is a close relative of $N$. crassa, is a model organism to study fruiting-body development. In recent years it turned out that S. macrospora is also well suitable to analyze the involvement of autophagy on complex developmental processes (Teichert et al. 2014). The filamentous ascomycete $S$. macrospora belongs to the order Sordariales. Under natural conditions, S. macrospora is a coprophilous fungus and grows on herbivore dung. It is self-fertile (homothallic) and lacks an asexual life cycle, meaning it solely undergoes a sexual life cycle without the need of a mating partner. In contrast to N. crassa, S. macrospora produces no asexual spores (Esser and Straub 1958; Pöggeler et al. 1997; Lord and Read 2011). Thus, mutations can be directly tested on their influence on fruiting-body formation (Teichert et al. 2014). The life cycle of $S$. macrospora is illustrated in Figure 7. The formation of multicellular fruiting bodies is an essential step in sexual reproduction of $S$. macrospora and involves highly controlled cellular differentiation programs (Pöggeler et al. 2006).

S. macrospora is a haplont with a genome of seven chromosomes ranging in size from 3.77.5 Mb (Pöggeler et al. 2000). It is suitable for genetic analysis because the genome of $39.8 \mathrm{Mb}$ is completely sequenced (Nowrousian et al. 2010).

Another advantage of $S$. macrospora is the formation of large ascospores $(31 \times 18 \mu \mathrm{m})$ (Walkey and Harvey 1966), which allow classical genetic experiments and the isolation of homokaryotic mutants after mutagenesis (Esser and Straub 1958; Teichert et al. 2014). Additional benefits of S. macrospora are its short life cycle of seven days and the easy handling and cultivation under laboratory conditions. S. macrospora is closely related to the heterothallic ascomycete $N$. crassa as evidenced by nucleic-acid identity of $90 \%$ within coding regions of orthologous genes (Nowrousian et al. 2010). 
The S. macrospora life cycle starts with a germinating ascospore and the formation of a two dimensional vegetative mycelium. The development of asci begins with the formation of female gametangia called ascogonia. Finally, the differentiation to fruiting-body precursors occurs which are called protoperithecia and later develops to mature fruiting bodies (perithecia). Karyogamy, meiosis, and postmeiotic mitosis occur in the perithecium and each perithecium harbors approximately 200 asci with eight linearly ordered ascospores. These are discharged by an increased turgor pressure at day seven to eight through an apical pore (ostiole) at the neck of the fruiting body (Engh et al. 2010; Lord and Read 2011) (Figure 7).

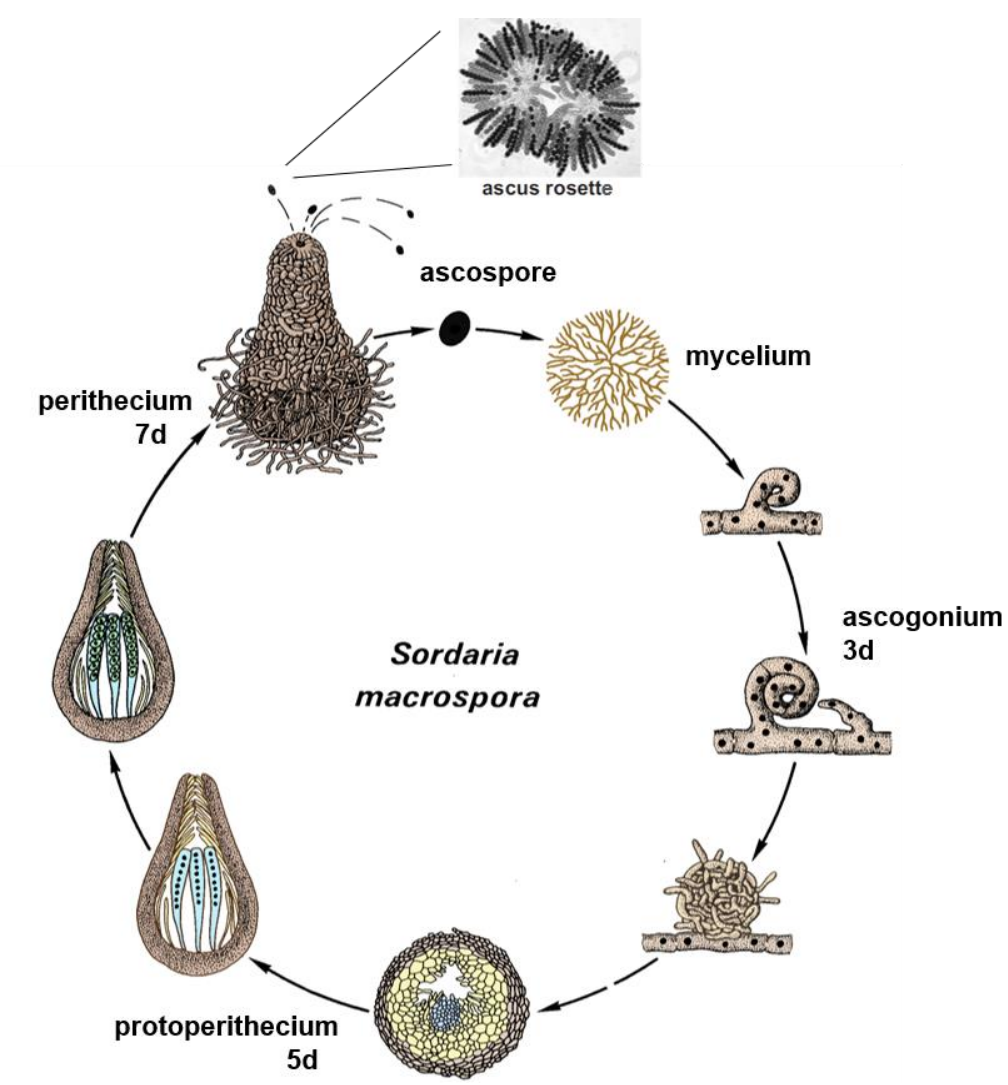

Figure 7: Life cycle of the homothallic ascomycete $S$. macrospora (Kück et al. 2009).

The cycle starts with the germination of an ascospore. After approximately three $d$ the female gametangia (ascogonium) starts to develop. The protoperithecium is the fruiting-body precursor and is formed by enveloping the ascogonium with sterile hyphae. After 4-5 d the young and round fruiting body develops into a pear-shaped mature fruiting body (perithecium) with approximately 200 asci each containing eight linearly ordered ascospores inside. The cycle ends with the discharge of the ascospores after approximately seven days.

All in all, the ascomycete $S$. macrospora is a good model system for higher eukaryotes to analyze multicellular development, meiosis and differentiation processes and also conserved processes like autophagy (Engh et al. 2010; Nowrousian et al. 2010; Teichert et al. 2014). 


\subsection{The autophagy process in $S$. macrospora}

The process of autophagy has been intensively investigated in yeast. However, not much is known in filamentous fungi. A BLASTP search in S. macrospora revealed 16 of the 17 core ATG proteins, as well as VPS34 and VPS15, and nine of the other 24 ATG proteins involved in induction and selective autophagy are conserved (Meijer et al. 2007a; Avin-Wittenberg et al. 2012; Voigt and Pöggeler 2013b) (Table 1).

Table 1: Conservation of atg genes in $S$. macrospora compared to yeasts.

\begin{tabular}{|c|c|c|c|}
\hline protein in yeasts & $\begin{array}{l}\text { protein in } \\
\text { S. macrospora }\end{array}$ & protein in yeasts & \begin{tabular}{|l|} 
protein in \\
S. macrospora
\end{tabular} \\
\hline \multicolumn{2}{|c|}{ core autophagy genes } & \multicolumn{2}{|c|}{ autophagy induction and nucleation } \\
\hline Atg1 & SMAC_02237 & $\operatorname{Atg} 11$ & SMAC_04225 \\
\hline Atg2 & SMAC_05551 & Atg17 & SMAC_04531 \\
\hline Atg3 & SMAC_05399 & $\operatorname{Atg} 29$ & \begin{tabular}{|l} 
SMAC_05468 \\
\end{tabular} \\
\hline $\operatorname{Atg} 4$ & SMAC_08321 & $\operatorname{Atg} 31$ & \begin{tabular}{|c|} 
not found \\
\end{tabular} \\
\hline $\operatorname{Atg} 5$ & SMAC_08343 & $\operatorname{Atg} 38$ & not found \\
\hline Atg6 & SMAC_02852 & $\operatorname{Atg} 41$ & not found \\
\hline Atg7 & SMAC_06539 & \multicolumn{2}{|l|}{ Cvt pathway } \\
\hline Atg8 & SMAC_02305 & Atg19 & not found \\
\hline Atg9 & SMAC_08312 & $\underline{\operatorname{Atg} 20}$ & SMAC_00076 \\
\hline$\underline{\operatorname{Atg} 10}$ & $\underline{\text { SMAC } 04841}$ & Atg21 & not found \\
\hline Atg12 & SMAC_06998 & $\operatorname{Atg} 23$ & not found \\
\hline Atg13 & SMAC_03472 & $\underline{\operatorname{Atg} 24}$ & SMAC 02936 \\
\hline Atg14 & not found & $\operatorname{Atg} 27$ & not found \\
\hline $\operatorname{Atg} 15$ & SMAC_02199 & $\operatorname{Atg} 34$ & not found \\
\hline$\underline{\operatorname{Atg} 16}$ & $\underline{\text { SMAC } 00084}$ & \multicolumn{2}{|l|}{ mitophagy } \\
\hline Atg18 & SMAC_09496 & $\operatorname{Atg} 32$ & not found \\
\hline $\operatorname{Atg} 22$ & SMAC_08052 & Atg33 & SMAC_03759 \\
\hline \multicolumn{2}{|l|}{ pexophagy } & \multicolumn{2}{|l|}{ ER/nucleophagy } \\
\hline Atg25\# & not found & Atg39 & not found \\
\hline Atg26 & SMAC_06693 & Atg40 & not found \\
\hline$\underline{\operatorname{Atg} 28 *}$ & SMAC_08301 & \\
\hline $\operatorname{Atg} 30 *$ & not found & & \\
\hline$\underline{\operatorname{Atg} 35^{*}}$ & SMAC 08301 & & \\
\hline$\overline{\operatorname{Atg} 36}$ & not found & & \\
\hline $\operatorname{Atg} 37 *$ & not found & & \\
\hline
\end{tabular}

41 atg genes have been identified in the yeasts S. cerevisiae, P. pastoris (*) and Pichia angusta (\#). By BLASTP search in S. macrospora, 16 of the 17 core ATG proteins and nine of the 24 proteins involved in the induction of autophagy and selective autophagy are conserved when compared with yeast homologs. Conserved proteins in S. macrospora are indicated with the SMAC number (e value, $\left.<\mathrm{e}^{-10}\right)$; similar proteins are underlined $\left(\mathrm{e}\right.$ value, $>\mathrm{e}^{-}$ $\left.{ }^{10}\right)$; not found, means no significant homolog in S. macrospora.

In the filamentous ascomycete $S$. macrospora previous studies have shown that autophagy is required for vegetative growth, the development of fruiting bodies and viability (Nolting et al. 
2009; Voigt et al. 2013; Voigt and Pöggeler 2013b; Voigt and Pöggeler 2013a; Teichert et al. 2014; Voigt et al. 2014).

In S. macrospora, the conservation of the core atg genes Smatg8 and Smatg4 was verified by the heterologous expression of SmATG8 and SmATG4 in S. cerevisiae atg 8 and atg4 deletion strain. Deletion of Smatg8 and Smatg4 leads to a lower germination efficiency, as well as to a sterile phenotype, underlining the importance of these proteins for fruiting-body development (Voigt and Pöggeler 2013a). These two proteins seem to be important for non-selective pexophagy as well, since the study from Voigt and Pöggeler (2013a) has shown that pexophagy is abolished in Smatg8 and Smatg4 deletion mutants.

Additionally, the E1-like enzyme SmATG7 was shown to be required for the viability of $S$. macrospora. It was not possible to generate a homokaryotic $\Delta$ Smatg7 mutant (Nolting et al. 2009).

Similarly, deletion of the vacuolar protein sorting genes Smvps34 and Smvps 15 prevented the isolation of homokaryotic knockout strains, showing that they are also required for viability of S. macrospora. In S. cerevisiae, VPS34 is the core protein of the complex I (ATG6, ATG14, VPS15 and VPS34), and complex II (ATG6, VPS38, VPS15 and VPS34), and important for protein sorting in the vacuole. Moreover, the PtdIns(3)P activity of VPS34 is required for Gprotein signaling and in the Tor nutrient-sensing (Voigt et al. 2014). VPS15 is the regulatory subunit and membrane targeting unit of VPS34 (Yan and Backer 2007).

To get a better understanding how autophagy is regulated, the function of the bZIP transcription factor SmJLB1, a homolog of the Podospora anserina basic zipper-type transcription factor I induced during incompatibility 4 (IDI4) and the Aspergillus nidulans transcription factor junlike bZIP A (JlbA), was analyzed. Deletion of Smjlbl impaired vegetative growth and fruitingbody development in S. macrospora. SmJLB1 acts as a repressor on gene expression of Smatg8 and Smatg4. SmJLB1 is localized to the cytoplasm and to nuclei (Voigt et al. 2013).

In conclusion, conserved core components of the autophagic machinery are involved in developmental processes and viability. 


\subsection{Aims of this thesis}

Autophagy is a major cellular degradation pathway conserved in eukaryotes which is mainly investigated in the baker's yeast S. cerevisiae. It permits the cells to eliminate unwanted proteins or organelles and to recycle components for reuse within a double-membraned autophagosome and vacuole. It has a great variety of physiological and pathophysiological roles in eukaryotic cells. In yeast, 41 atg genes exist and the ubiquitin-like gene Smatg 12 represents one of the conserved core autophagy genes. Up to now, its function was not analyzed in filamentous fungi. The deletion mutant of $\Delta \operatorname{Smatg} 12$ will be analyzed regarding its function in vegetative growth and sexual development. In case of phenotypical alterations it should be rescued in complementation experiments using an egfp-Smatg12 fusion construct. The complemented strain will be used for in vivo localization studies of EGFP-SmATG12 in S. macrospora. Furthermore, the functional conservation of SmATG12 and its yeast counterpart will be analyzed by a yeast complementation assay with the Smatg12 cDNA expressed under the control of a MET25 promoter in an S. cerevisiae atg12 $\Delta$ null mutant. Rescue of autophagy in the S. cerevisiae atg12 mutant will be monitored using an aminopeptidase I (Ape1) maturation assay based on the autophagy-dependent maturation of the precursor proaminopetidase I (prApe1) to the mature enzyme (mApe1).

Selective autophagy is characterized by cargo receptor proteins, which are able to bind a specific cargo including organelles, proteins or microbes for their autophagic degradation. In addition to SmATG12, SmATG8 represents another core autophagy protein, which is necessary for autophagosome formation (Voigt and Pöggeler 2013a).

GFP-Trap analysis followed by liquid chromatography mass spectrometry (LC/MS) using SmATG8 as bait identified SmNBR1 as putative interaction partner of SmATG8 (Voigt 2012). SmNBR1 is a putative homolog of the human autophagy cargo receptor NBR1.

To determine whether selective autophagy is necessary for vegetative growth and fruiting-body development in S. macrospora, the function of the putative cargo receptor SmNBR1 should be analyzed. For this, a $\Delta$ Smnbrl mutant strain as well as a complementation strain should be constructed and phenotypically analyzed under stress-inducing conditions. NBR1 proteins display a conserved domain architecture. Therefore, a complementation of the $\Delta$ Smnbr1 mutant with the H. sapiens NBR1 homolog is planned. In mammals and plants an interaction between ATG8 and NBR1 has been described (Johansen and Lamark 2011; Svenning et al. 2011). For this reason, the proteins SmATG8 and SmNBR1 should be co-localized in S. macrospora by fluorescence microscopy and their interaction should be verified by Bimolecular fluorescence 


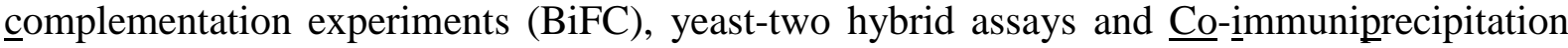
experiments (Co-IP).

Mutations and truncated constructs of SmNBR1 should be constructed and tested for their complementation ability of $\Delta$ Smnbrl and should be localized regarding to the formation of autophagosomes and their vacuolar localization.

Since it is known that NBR1 is involved in the turnover of endogenous peroxisomes in mammals (Deosaran et al. 2013), the function of SmNBR1 in the autophagic degradation of peroxisomes, called pexophagy, should be investigated. For this, the localization of peroxisomal proteins will be analyzed in $\Delta$ Smnbr1 and the autophagy-defective mutant $\Delta$ Smatg8 and compared to the localization observed in wild type. With regard to the function of peroxisomes, including the $\beta$-oxidation of fatty acids and detoxification of reactive oxygen species, the growth on media containing long-chain fatty acids as sole carbon source will be tested in the $\Delta$ Smnbr1 strain as well as the growth on media containing $\mathrm{H}_{2} \mathrm{O}_{2}$. Woronin bodies are unique organelles present in Pezizomycotina (Maruyama et al. 2005; Jedd 2011), which arise from peroxisomes. Therefore the function of Woronin bodies will also be analyzed in the $\Delta$ Smnbr1 mutant strain. Additionally, putative cargos of SmNBR1 should be identified by GFPTrap and LC/MS analysis using SmNBR1 as bait.

Ribophagy describes the selective autophagic degradation of ribosomes. Since the selectivity receptor for ribophagy is unknown in yeast and in higher eukaryotes it remains to be investigated in more detail how ribosomes are subjected to autophagy. It is unknown how intact ribosomal subunits are recognized by the receptor and how ribophagy is regulated in eukaryotes. Therefore, ribosomal proteins of either the large $60 \mathrm{~S}$ ribosomal subunit or a small $40 \mathrm{~S}$ ribosomal subunit protein should be analyzed in the $\Delta$ Smnbr1 strain. The EGFP-labeled large and small ribosomal proteins will be localized to follow their degradation in the vacuole. Furthermore, the proteins also should be studied by quantitative Western-blot experiments. 


\section{Materials and Methods}

\subsection{Materials}

\subsubsection{Strains}

Table 2: Overview of strains used and constructed in this study.

\begin{tabular}{lll}
\hline Strain & Genotype & Source \\
\hline & \multicolumn{1}{c}{ Escherichia coli } \\
MACH1 & $\begin{array}{ll}\Delta r e c A 1398, \text { endA1, tonA, } \Phi 804 l a c M 15, \\
\Delta l a c X 74, \text { hsdR, (rK- } \mathrm{mK}+)\end{array}$ & Invitrogen
\end{tabular}

\section{Saccharomyces cerevisiae}

PJ69-4A

AH109

Y187

BY4741

$\operatorname{atg} 12 \Delta$
MATa, trp1-901, leu2-3,112, ura3-52, his3-200, ga14L, gal8OD LYS2::GALlHIS3, GAL2-ADE2, met2::GAL7-lacZ

MATa, trp1-901, leu2-3, 112, ura3-52, his3-200, ade2-101, gal44, gal804, lys2::GallUAS-GallTATAhis3, Gal2UASGal2TATA ade2, ura3::MellUAS-MellTATA lacZ, Mel1

MATa, ura3-52, his3-200, ade2101, trp1-901, leu2-3, 112,

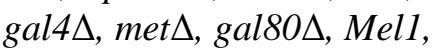
ura3::GallUAS-Gal1TATA lacZ

$M A T a$, his $3 \Delta 1$, leu $2 \Delta 0$, ura $3 \Delta 0$, met $15 \Delta 0$
(James et al. 1996)

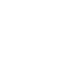




\begin{tabular}{|c|c|c|}
\hline$\Delta$ Smnbr1 & $\Delta$ Smnbr1::hyg ${ }^{\mathrm{R}}$, ssi, sterile & This study \\
\hline$\Delta \operatorname{Smatg} 12$ & $\Delta$ Smatg12::hyg ${ }^{\mathrm{R}}$, ssi, sterile & (Werner et al. 2016) \\
\hline$\Delta$ Smatg8 & $\Delta$ Smatg8::hyg ${ }^{\mathrm{R}}$, ssi, sterile & $\begin{array}{l}\text { (Voigt and Pöggeler } \\
\text { 2013a) }\end{array}$ \\
\hline$\Delta$ Smubp3 & $\Delta$ Smubp3::hyg ${ }^{\mathrm{R}}$, ssi, sterile & $\begin{array}{l}\text { Herzog pers. } \\
\text { comm., Göttingen }\end{array}$ \\
\hline $\mathrm{wt}:: 1783-1^{\mathrm{ect}}\left(\mathrm{wt}::\right.$ egfp $\left.{ }^{\mathrm{ect}}\right)$ & $h y g^{\mathrm{R}}, e g f p^{\mathrm{ect}}, \mathrm{ssi}$ & This study \\
\hline $\mathrm{wt}:: \mathrm{RHN} 1^{\mathrm{ect}}$ & $n a t^{\mathrm{R}}$, Dsred ${ }^{\mathrm{ect}}, \mathrm{ssi}$ & This study \\
\hline $\begin{array}{l}\text { wt::Smnbr1-Dsred }{ }^{\text {ect }}+ \\
\text { egfp-Smatg } 8^{\text {ect }}\end{array}$ & $\begin{array}{l}\text { wt, Smnbrl-Dsred }{ }^{\text {ect }}, h y g^{\mathrm{R}}, \text { egfp- } \\
\text { Smatg8 }{ }^{\text {ect }}, \text { nat }^{\mathrm{R}} \text {, ssi }\end{array}$ & This study \\
\hline $\mathrm{wt}::$ Smnbr1-YC $C^{\mathrm{ect}}+\mathrm{YN}-\mathrm{Smatg} 8^{\mathrm{ect}}$ & $\begin{array}{l}\text { wt, Smnbrl-eyfp-c } c^{\mathrm{ect}}, n a t^{\mathrm{R}}, \text { eyfp- } n- \\
\text { Smatg }{ }^{\mathrm{ect}}, \text { hyg }^{\mathrm{R}}, \mathrm{ssi}\end{array}$ & This study \\
\hline $\mathrm{wt}:: S m n b r 1-\mathrm{YC}^{\mathrm{ect}}+\mathrm{YN}^{\mathrm{ect}}$ & $\begin{array}{l}\text { wt, Smnbrl-eyfp-c }{ }^{\mathrm{ect}}, n a t^{\mathrm{R}}, \text { eyfp- } n^{\mathrm{ect}} \\
\text { hyg }^{\mathrm{R}} \text {, ssi }\end{array}$ & This study \\
\hline $\mathrm{wt}:: \mathrm{YC}^{\mathrm{ect}}+\mathrm{YN}-\mathrm{Smatg} 8^{\mathrm{ect}}$ & $\begin{array}{l}\text { wt, eyfp-c } c^{\mathrm{ect}}, n a t^{\mathrm{R}}, \text { eyfp- } n \text {-Smatg } 8^{\mathrm{ect}} \\
h y g^{\mathrm{R}}, \mathrm{ssi}\end{array}$ & This study \\
\hline $\mathrm{wt}::$ Smnbr1-YC ${ }^{\mathrm{ect}}$ & wt, Smnbrl-eyfp-c $c^{\mathrm{ect}}, n a t^{\mathrm{R}}$, ssi & This study \\
\hline $\mathrm{wt}:: \mathrm{YN}-\mathrm{Smatg} 8^{\mathrm{ect}}$ & wt, eyfp-n-Smatg $8^{\text {ect }}, h y g^{\mathrm{R}}$, ssi & This study \\
\hline wt::Smrpl25-egfp ${ }^{\text {ect }}$ & wt, Smrpl25-egfp $p^{\mathrm{ect}}, n a t^{\mathrm{R}}$, ssi & This study \\
\hline $\mathrm{wt}::$ Smrps19-egfp ${ }^{\text {ect }}$ & wt, Smrps19-egfp $p^{\mathrm{ect}}, n a t^{\mathrm{R}}$, ssi & This study \\
\hline $\begin{array}{l}\text { wt::Smnbr1-Dsred } \\
\text { egfp }^{\text {ect }}+\text { Smrpl25- }\end{array}$ & $\begin{array}{l}\text { wt, Smnbrl-Dsred }{ }^{\text {ect }}, h y g^{\mathrm{R}}, \text { Smrpl25- } \\
\text { egfp }^{\text {ect }}, n a t^{\mathrm{R}}, \text { ssi }\end{array}$ & This study \\
\hline $\begin{array}{l}\text { wt::Smnbr1-Dsred } \\
\text { egfp }{ }^{\text {ect }}+\text { Smrps 19- }\end{array}$ & $\begin{array}{l}\text { wt, Smnbrl-Dsred } \\
\text { egfp }{ }^{\text {ect }}, \text { hat }^{\mathrm{R}}, \text { ssi }\end{array}$ & This study \\
\hline wt::Smpex14-egfp ${ }^{\text {ect }}$ & wt, Smpex14-egfp $p^{\text {ect }}, n a t^{\mathrm{R}}$, ssi & This study \\
\hline $\begin{array}{l}\text { wt::Smnbr1-Dsred }{ }^{\text {ect }}+\text { Smpex14- } \\
\text { egfp }\end{array}$ & $\begin{array}{l}\text { wt, Smnbrl-Dsred }{ }^{\text {ect }}, h y g^{\mathrm{R}}, \text { Smpex14- } \\
\text { egfp }^{\text {ect }}, n a t^{\mathrm{R}} \text {, ssi }\end{array}$ & This study \\
\hline $\mathrm{wt}:$ :Dsred-Smhex $1^{\text {ect }}$ & wt, Dsred-Smhex $1^{\text {ect }}$, nat $^{\mathrm{R}}$, ssi & This study \\
\hline wt::Dsred-SKL ${ }^{\text {ect }}+$ Smnbr1-egfp ${ }^{\text {ect }}$ & $\begin{array}{l}\text { wt, Dsred-SKL } L^{\mathrm{ect}}, n a t^{\mathrm{R}}, \text { Smnbrl-egfp } \\
h y g^{\mathrm{R}} \text {, ssi }\end{array}$ & This study \\
\hline$\Delta$ Smnbr1::egfp ${ }^{\text {ect }}$ & $\begin{array}{l}\Delta \text { Smnbr1::hyg }{ }^{\mathrm{R}}, e g f p^{\text {ect }} \\
n a t^{\mathrm{R}}, \text { ssi }\end{array}$ & This study \\
\hline$\Delta$ Smnbr1::Smnbr1-egfp ${ }^{\text {ect }}$ & $\begin{array}{l}\Delta \text { Smnbr1::hyg } g^{\mathrm{R}}, \text { Smnbrl-egfp } \\
n a t^{\mathrm{R}}, \text { ssi }\end{array}$ & This study \\
\hline
\end{tabular}




\author{
$\Delta$ Smnbr1::Smnbr1-Dsred ${ }^{\text {ect }}$ \\ $\Delta$ Smnbr1::Smnbr1-1-418-egfp ${ }^{\text {ect }}$ \\ $\Delta$ Smnbr1::Smnbr1-419-863-egfp ${ }^{\text {ect }}$ \\ $\Delta$ Smnbr1::Smnbr1-1-843-egfp ${ }^{\text {ect }}$ \\ $\Delta$ Smnbr1::Smnbr1-Y ${ }^{847}$ A $\mathrm{L}^{850} \mathrm{~A}-$ \\ egfp $^{\text {ect }}$ \\ $\Delta$ Smnbr1::Smnbr1-T ${ }^{843}$ A-egfp ${ }^{\text {ect }}$ \\ $\triangle$ Smnbr1::Smnbr1-T ${ }^{843}$ A $\mathrm{Y}^{847} \mathrm{~A}$ \\ $\mathrm{L}^{850} \mathrm{~A}-\mathrm{egf} \mathrm{p}^{\text {ect }}$ \\ $\triangle$ Smnbr1::Smnbr1-S ${ }^{793}$ A S $^{804} \mathrm{~A}$ \\ $\mathrm{S}^{808}$ A-egfp ${ }^{\text {ect }}$ \\ $\Delta$ Smnbr1::ccg1-Smnbr1-egfp ${ }^{\text {ect }}$ \\ $\Delta$ Smnbr1::egfp-Smatg8 $8^{\text {ect }}$ \\ $\Delta$ Smnbr1:: Smrpl25-egfp ${ }^{\text {ect }}$
}

$\Delta$ Smnbr1:: Smrps19-egfp ${ }^{\text {ect }}$

$\Delta$ Smnbr1::Smpex 14-egfp ect $^{\text {ST }}$

$\Delta$ Smnbr1::Dsred-Smhex $1^{\text {ect }}$

$\Delta$ Smnbr1::Dsred-SKL ${ }^{\text {ect }}$

$\Delta$ Smnbr1::Hsnbr1-Dsred ${ }^{\text {ect }}$

$\Delta$ Smatg12::Smnbr1-Dsred ${ }^{\text {ect }}$

$\Delta$ Smatg12::egfp-Smatg12 ect

$\Delta$ Smatg12::egfp-Smatg8 ${ }^{\text {ect }}$
$\Delta$ Smnbr1::hyg ${ }^{\mathrm{R}}$, Smnbrl-Dsred ${ }^{\mathrm{ect}}$, $n a t^{\mathrm{R}}$, ssi

$\Delta$ Smnbr1::hyg ${ }^{\mathrm{R}}$, Smnbrl aa 1-418$e g f p^{\text {ect }}, n a t^{\mathrm{R}}$, ssi

This study

$\Delta$ Smnbr1::hyg ${ }^{\mathrm{R}}$, Smnbrl aa 419-863$e g f p^{\text {ect }}, n a t^{\mathrm{R}}, \mathrm{ssi}$

$\Delta$ Smnbr1::hyg ${ }^{\mathrm{R}}$, Smnbrl aa 1-843$e g f p^{\mathrm{ect}}, n a t^{\mathrm{R}}, \mathrm{ssi}$

$\triangle$ Smnbr1::hyg ${ }^{\mathrm{R}}$, Smnbrl- $Y^{847} A L^{850} A-$ $e g f p^{\mathrm{ect}}, n a t^{\mathrm{R}}, \mathrm{ssi}$

$\Delta$ Smnbr1::hyg $g^{\mathrm{R}}$, Smnbrl- $T^{843} A-e g f p^{\mathrm{ect}}$, $n a t^{\mathrm{R}}, \mathrm{ssi}$

$\triangle$ Smnbr1::hyg $g^{\mathrm{R}}$, Smnbrl- $T^{843} A Y^{847} A \quad$ This study $L^{850} A-e g f p^{\text {ect }}$, nat $^{\mathrm{R}}$, ssi

$\Delta$ Smnbr1::hyg $g^{\mathrm{R}}$, Smnbrl $-S^{793} A S^{804} A \quad$ This study $S^{808} A-e g f p^{\text {ect }}, n a t^{\mathrm{R}}$, ssi

$\Delta$ Smnbr1::hyg $g^{\mathrm{R}}$, Smnbrl-egfp $p^{\mathrm{ect}}, \quad$ This study overexpression promoter $\operatorname{ccg} \mathrm{l}, n_{\mathrm{at}}^{\mathrm{R}}$, ssi

$\Delta$ Smnbr1::hyg $g^{\mathrm{R}}$, egfp-Smatg $8^{\mathrm{ect}}$, nat $^{\mathrm{R}}$, ssi $\quad$ This study $\Delta$ Smnbr1::hyg $g^{\mathrm{R}}$, Smrpl25-egfp ${ }^{\mathrm{ect}}, n a t^{\mathrm{R}}, \quad$ This study ssi

$\Delta$ Smnbr1::hyg $g^{\mathrm{R}}$, Smrps 19-egfp ${ }^{\mathrm{ect}}$, nat $^{\mathrm{R}}, \quad$ This study ssi

$\Delta$ Smnbr1::hyg $g^{\mathrm{R}}$, Smpex14-egfp ${ }^{\mathrm{ect}}$, nat $^{\mathrm{R}}, \quad$ This study ssi

$\Delta$ Smnbr1::hyg ${ }^{\mathrm{R}}$, Dsred-Smhex $1^{\mathrm{ect}}$, nat $^{\mathrm{R}}, \quad$ This study ssi

$\Delta$ Smnbr1::hyg ${ }^{\mathrm{R}}$, Dsred-SKL ${ }^{\mathrm{ect}}$, nat $^{\mathrm{R}}$, ssi $\quad$ This study

$\Delta$ Smnbr1::hyg ${ }^{\mathrm{R}}$, Hsnbrl-Dsred ${ }^{\mathrm{ect}}$, nat $^{\mathrm{R}}$, This study $\mathrm{ssi}$

$\Delta$ Smatg12::hyg ${ }^{\mathrm{R}}$, Smnbrl-Dsred ${ }^{\mathrm{ect}}, \quad$ This study $n a t^{\mathrm{R}}, \mathrm{ssi}$

$\Delta$ Smatg12::hyg ${ }^{\mathrm{R}}$, egfp-Smatg $12^{\text {ect }}$, $n a t^{\mathrm{R}}$, ssi

(Werner et al. 2016)

$\Delta$ Smatg $12: \because h y g^{\mathrm{R}}$, egfp-Smatg $8^{\mathrm{ect}}$, $n a t^{\mathrm{R}}$, ssi

(Werner et al. 2016) 


\begin{tabular}{|c|c|c|}
\hline$\Delta$ Smatg8:: Smnbr1-Dsred ${ }^{\text {ect }}$ & $\begin{array}{l}\Delta \text { Smatg8::hyg }{ }^{\mathrm{R}}, \text { Smnbrl-Dsred } \\
n a t^{\mathrm{R}}, \text { ssi }\end{array}$ & This study \\
\hline$\Delta$ Smatg8::egfp-Smatg8 $8^{\text {ect }}$ & $\begin{array}{l}\Delta \mathrm{Smatg} 8:: h y g^{\mathrm{R}}, \text { egfp-Smatg } 8^{\mathrm{ect}} \\
n a t^{\mathrm{R}}, \mathrm{ssi}\end{array}$ & $\begin{array}{l}\text { (Voigt and Pöggeler } \\
\text { 2013a) }\end{array}$ \\
\hline$\Delta$ Smatg8::egfp-Smatg12 ${ }^{\text {ect }}$ & $\begin{array}{l}\Delta \mathrm{Smatg} 8:: h y g^{\mathrm{R}}, \text { egfp-Smatg } 12^{\mathrm{ect}}, n a t^{\mathrm{R}}, \\
\mathrm{ssi}\end{array}$ & (Werner et al. 2016) \\
\hline$\Delta$ Smatg8:: Smrpl25-egfp ${ }^{\text {ect }}$ & $\begin{array}{l}\Delta \text { Smatg8::hyg } g^{\mathrm{R}}, \text { Smrpl25-egfp } \\
\text { ssi }\end{array}$ & This study \\
\hline$\Delta$ Smatg8::Smpex14-egfp ${ }^{\text {ect }}$ & $\begin{array}{l}\Delta \text { Smatg8::hyg } g^{\mathrm{R}}, \text { Smpex14-egfp } \\
\text { ssi }\end{array}$ & This study \\
\hline$\Delta$ Smatg8::Dsred-Smhex $1^{\text {ect }}$ & $\begin{array}{l}\Delta \text { Smatg8::hyg }{ }^{\mathrm{R}}, \text { Dsred-Smhex }{ }^{\mathrm{ect}}, \text { nat }^{\mathrm{R}} \text {, } \\
\text { ssi }\end{array}$ & This study \\
\hline$\Delta$ Smatg8:: Smrps19-egfp ${ }^{\text {ect }}$ & $\begin{array}{l}\Delta \text { Smatg8::hyg }{ }^{\mathrm{R}}, \text { Smrps 19-egfp } \\
\text { ssi }\end{array}$ & This study \\
\hline$\Delta$ Smatg4::egfp-Smatg $8^{\text {ect }}$ & $\begin{array}{l}\Delta \text { Smatg } 4:: h y g^{\mathrm{R}}, \text { egfp-Smatg } 8^{\mathrm{ect}} \\
n a t^{\mathrm{R}}, \text { ssi }\end{array}$ & $\begin{array}{l}\text { (Voigt and Pöggeler } \\
\text { 2013a) }\end{array}$ \\
\hline
\end{tabular}

$\overline{n a t^{\mathrm{R}}}$, nourseothricin resistant; $h y g^{\mathrm{R}}$, hygromycin resistant; ssi, single spore isolate; ect, ectopical integrated.

\subsubsection{Plasmids}

Table 3: Overview of the used and generated plasmids in this study.

\begin{tabular}{|c|c|c|}
\hline Plasmid & Characteristics & Source \\
\hline \multicolumn{3}{|c|}{ general usage } \\
\hline pCB1003 & $a m p^{\mathrm{R}}, h p h$-cassette & (Carroll et al. 1994) \\
\hline pRS426 & $U R A 3, a m p^{\mathrm{R}}$ & $\begin{array}{l}\text { (Christianson et al. } \\
\text { 1992) }\end{array}$ \\
\hline pRS426-met25 & MET25 promoter, $U R A 3, a m p^{\mathrm{R}}$ & (Mumberg et al. 1994) \\
\hline pRSnat & $U R A 3, a m p^{\mathrm{R}}, n a t^{\mathrm{R}}$ & (Klix et al. 2010) \\
\hline pRShyg & $U R A 3, a m p^{\mathrm{R}}, h y g^{\mathrm{R}}$ & (Bloemendal et al. 2012) \\
\hline p1783-1 & $\begin{array}{l}\text { egfp under control of } g p d \text { promoter and } \\
\operatorname{trp} C \text { terminator, } a m p^{\mathrm{R}}, h y g^{\mathrm{R}}\end{array}$ & (Pöggeler et al. 2003) \\
\hline pDS23 & $\begin{array}{l}\text { egfp under control of } g p d \text { promoter and } \\
\operatorname{trp} C \text { terminator, } a m p^{\mathrm{R}}, n a t^{\mathrm{R}}\end{array}$ & (Teichert et al. 2012) \\
\hline pRHN1 & $\begin{array}{l}\text { Dsred under control of } g p d \text { promoter and } \\
\operatorname{trp} C \text { terminator, } a m p^{\mathrm{R}}, n a t^{\mathrm{R}}\end{array}$ & (Janus et al. 2007) \\
\hline
\end{tabular}




\begin{tabular}{|c|c|c|}
\hline pHAN1 & $\begin{array}{l}\text { egfp under control of } c c g l \text { overexpression } \\
\text { promoter, } a m p^{\mathrm{R}}, h y g^{\mathrm{R}}\end{array}$ & (Kawabata et al. 2007) \\
\hline pDsred-SKL & $\begin{array}{l}S K L \text { under control of } g p d \text { promoter and } \\
\operatorname{trp} C \text { terminator of } A \text {. nidulans, } a m p^{\mathrm{R}}, n a t^{\mathrm{R}}\end{array}$ & $\begin{array}{l}\text { (Elleuche and Pöggele } \\
\text { 2008) }\end{array}$ \\
\hline pEYFPN & $\begin{array}{l}\text { N-terminal part of eyfp under control of } \\
g p d \text { promoter, } a m p^{\mathrm{R}}, h y g^{\mathrm{R}}\end{array}$ & (Hoff and Kück 2005) \\
\hline pEYFPC & $\begin{array}{l}\text { C-terminal part of eyfp under control of } \\
g p d \text { promoter, } a m p^{\mathrm{R}}, h y g^{\mathrm{R}}\end{array}$ & (Hoff and Kück 2005) \\
\hline pRS-YC & $\begin{array}{l}\text { C-terminal part of eyfp under control of } \\
\text { gpd promoter, } U R A 3, a m p^{\mathrm{R}}, n a t^{\mathrm{R}}\end{array}$ & This study \\
\hline pRS-YN & $\begin{array}{l}\text { N-terminal part of eyfp under control of } \\
g p d \text { promoter } U R A 3, a m p^{\mathrm{R}}, h_{y} g^{\mathrm{R}}\end{array}$ & This study \\
\hline pOTB7+cDNA & $c m^{\mathrm{R}}, H s n b r l \mathrm{cDNA}$ & $\begin{array}{l}\text { transOMIC } \\
\text { technologies } \\
\text { BC009808 }\end{array}$ \\
\hline \multicolumn{3}{|c|}{ localization studies and knockouts } \\
\hline pSmnbr1-KO & $\begin{array}{l}1050 \text { bp of the } 5 \text { '-flanking region and } 785 \\
\text { bp of 3'-flanking region of Smnbrl } \\
\text { interrupted by the } h p h \text {-cassette in pRS } 426\end{array}$ & This study \\
\hline pSmatg12-KO & $\begin{array}{l}1058 \text { bp of the } 5 \text { '-flanking region and } 700 \\
\text { bp of 3'-flanking region of Smatg } 12 \\
\text { interrupted by the } h \text { ph-cassette in pRS } 426\end{array}$ & (Werner et al. 2016) \\
\hline pSmnbr1-egfp & $\begin{array}{l}991 \text { bp of the } 5 \text {-flanking region and } 2681 \\
\text { bp of the ORF of Smnbrl without stop } \\
\text { codon, egfp and trpC terminator ( } 1506 \mathrm{bp} \text { ) } \\
\text { in pRSnat }\end{array}$ & This study \\
\hline pSmnbr1-Dsred & $\begin{array}{l}991 \text { bp of the } 5 \text {-flanking region and } 2681 \\
\text { bp of the ORF of Smnbrl without stop } \\
\text { codon, Dsred and trpC terminator ( } 1466 \\
\text { bp) in pRShyg or pRSnat }\end{array}$ & This study \\
\hline pccg1-Smnbr1-egfp & $\begin{array}{l}921 \text { bp of } c c g 1 \text { promoter region and } 2681 \\
\text { bp of } S m n b r 1 \text { ORF without stop codon, } \\
\text { egfp and trpC terminator ( } 1506 \text { bp) in } \\
\text { pRSnat }\end{array}$ & This study \\
\hline pSmnbr1-1-418-egfp & $\begin{array}{l}991 \text { bp of the } 5 \text {-flanking region and } 1346 \\
\text { bp ORF of Smnbrl without stop codon, } \\
\text { egfp and trpC terminator ( } 1506 \text { bp) in } \\
\text { pRSnat }\end{array}$ & This study \\
\hline
\end{tabular}


pSmnbr1-419-863-egfp

pSmnbr1-1-843-egfp

pSmnbr1-Y ${ }^{847} A^{L^{850}} \mathrm{~A}$-egfp

pSmnbr1-T ${ }^{843} A-e g f p$

pSmnbr1-T ${ }^{843} \mathrm{~A} \mathrm{Y}^{847} \mathrm{~A} \mathrm{~L}^{850} \mathrm{~A}$-egfp

pSmnbr1-S ${ }^{793}$ A $S^{804}$ A $S^{808} A$-egfp

pSmnbr1-YC

pYN-Smatg8

pRS-egfp-Smatg8

pegfp-Smatg12
$991 \mathrm{bp}$ of the 5'-flanking region and 1332 This study bp ORF of Smnbrl without stop codon, egfp and trpC terminator (1506 bp) in pRSnat

$991 \mathrm{bp}$ of the 5 '-flanking region and 2621 bp of the ORF of Smnbrl without stop codon, egfp and trpC terminator (1506 bp) in pRSnat

$991 \mathrm{bp}$ of the 5'-flanking region and 2681 bp of the ORF of Smnbrl inserted mutations in $\mathrm{Y}^{847} \mathrm{~A} \mathrm{~L}^{850} \mathrm{~A}$ without stop codon, egfp and trpC terminator (1506 bp) in $\mathrm{pRSnat}$

$991 \mathrm{bp}$ of the 5'-flanking region and 2681 bp of the ORF of Smnbrl inserted mutations in $\mathrm{T}^{843} \mathrm{~A}$ without stop codon, egfp and $\operatorname{trp} C$ terminator (1506 bp) in pRSnat

$991 \mathrm{bp}$ of the 5'-flanking region and 2681 bp of the ORF of Smnbrl inserted mutations in $\mathrm{T}^{843} \mathrm{~A} \mathrm{Y}^{847} \mathrm{~A} \mathrm{~L}^{850} \mathrm{~A}$ without stop codon, egfp and $\operatorname{trp} C$ terminator (1506 bp) in pRSnat

$991 \mathrm{bp}$ of the 5'-flanking region and 2681 bp of the ORF of Smnbrl inserted mutations in $\mathrm{S}^{793} \mathrm{~A} \mathrm{~S}^{804} \mathrm{~A} \mathrm{~S}^{808} \mathrm{~A}$ without stop codon, egfp and trpC terminator (1506 bp) in pRSnat

706 bp gpd promoter and 2681 bp of Smnbrl ORF without stop codon, Cterminal part of $y f p$ (306 bp), trpC terminator (765 bp) in pRS-YC

706 bp gpd promoter, N-terminal part of $y f p$ (491 bp) and 503 bp of Smatg8 ORF with stop codon, $\operatorname{trp} C$ terminator (765 bp) in $\mathrm{pRS}-\mathrm{YN}$

$1023 \mathrm{bp}$ of the 5'-flanking region of Smatg8, egfp exluding stop codon, Smatg8 ORF without start codon and 1021 bp of 3'-flanking region of Smatg8 in pRSnat

$1058 \mathrm{bp}$ of the 5'-flanking region of Smatg12, egfp exluding stop codon, Smatg12 ORF without start codon and 1615 bp of 3'-flanking region of Smatg 12 in pRSnat
This study

This study

This study

This study

This study

This study

This study

(Voigt and Pöggeler 2013a)

(Werner et al. 2016) 
pHsnbr1-Dsred

pSmpex14-egfp

pDsred-Smhex 1

pSmrpl25-egfp

pSmrps19-egfp
$991 \mathrm{bp}$ of the 5 '-flanking region of

Smnbrl, 2810 bp of cDNA of the Hsnbrl

ORF without stop codon, Dsred and trpC terminator (1466 bp) in pRSnat

$794 \mathrm{bp}$ of the 5'-flanking region and 1250 bp of the ORF of Smpex 14 without stop codon, egfp and trpC terminator (1506 bp) in pRSnat

840 bp of the promoter trpC, Dsred (674 bp), 703 bp of Smhexl ORF and 849 bp of the 3 '-flanking region in $\mathrm{pRSnat}$

$1000 \mathrm{bp}$ of the 5'-flanking region and 686 bp of the ORF of Smrpl25 without stop codon, egfp and trpC terminator (1506 bp) in $\mathrm{pRSnat}$

722 bp of the 5'-flanking region and 861 bp of the ORF of Smrps19 without stop codon, egfp and trpC terminator (1506 bp) in pRSnat
This study

This study

This study

This study

This study

\section{yeast-two hybrid and maturation assay}

pGADT7

pGBKT7

pAD-ranBPM

pAD-Smatg8

pBD-Smatg8

pAD-Smatg12

pBD-Smatg12

pAD-Smatg7

pBD-Smatg7

pAD-Smatg3

pBD-Smatg3

pAD-Smnbr1
$L E U 2, a d h 1^{\mathrm{P}}, a d h 1^{\mathrm{T}}, G A L 4-A D, a m p^{\mathrm{R}}$, HA tag

$T R P 1, a d h 1^{\mathrm{P}}, a d h 1^{\mathrm{T}}, G A L 4-B D, k a n^{\mathrm{R}}$, myc tag

ran $\mathrm{BPM}$ in $\mathrm{pGADT7}$

Smatg8 cDNA in pGADT7

Smatg 8 cDNA in pGBKT7

Smatg 12 cDNA in pGADT7

Smatg12 cDNA in pGBKT7

Smatg7 cDNA in pGADT7

Smatg7 cDNA in pGBKT7

Smatg3 cDNA in in pGADT7

Smatg3 cDNA in pGBKT7

Smnbrl cDNA in pGADT7
Clontech laboratories inc. (Saint-Germain-en-

Laye, France), 630442

Clontech laboratories inc. (Saint-Germain-enLaye, France), 630443

(Tucker et al. 2009)

(Voigt and Pöggeler 2013a)

(Voigt and Pöggeler 2013a)

(Werner et al. 2016)

(Werner et al. 2016)

(Werner et al. 2016)

(Werner et al. 2016)

(Werner et al. 2016)

(Werner et al. 2016)

This study 
pBD-Smnbr1

pAD-Smnbr1(1-418)

pAD-Smnbr1(419-863)

pAD-Smnbr1(1-843)

pAD-Smnbr1 $\left(\mathrm{Y}^{847} \mathrm{~A} \mathrm{~L}{ }^{850} \mathrm{~A}\right)$

pAD-Smnbr1 $\left(\mathrm{T}^{843} \mathrm{~A}\right)$

pAD-Smnbr1 $\left(\mathrm{T}^{843} \mathrm{~A} \mathrm{Y}^{847} \mathrm{~A} \mathrm{~L}^{850} \mathrm{~A}\right)$

pAD-Smnbr1 $\left(\mathrm{S}^{793} \mathrm{~A} \mathrm{~S}^{804} \mathrm{~A} \mathrm{~S}^{808} \mathrm{~A}\right)$

pAD-Smnbr1 $\left(\mathrm{S}^{793} \mathrm{~A}\right)$

pAD-Smrpl25

pBD-Smrpl25

pAD-Smrps19

pBD-Smrps 19

pRS-met25-Smatg12

pRS-met25-Scatg12

pRS315-GFP-ScAtg8
Smnbrl cDNA in pGBKT7

Smnbrl aa 1-418 cDNA in pGADT7

Smnbrl aa 419-863 cDNA in pGADT7

Smnbrl aa 1-843 cDNA in pGADT7

Smnbrl $Y^{847} A L^{850} A$ cDNA in pGADT7

Smnbrl $T^{843} A$ cDNA in pGADT7

Smnbrl $T^{843} A Y^{847} A L^{850} A$ cDNA in pGADT7

Smnbrl $S^{793} A S^{804} A S^{808} A$ cDNA in pGADT7

Smnbrl $S^{793} A$ cDNA in pGADT7

Smrpl25 cDNA in pGADT7

Smrpl25 cDNA in pGBKT7

Smrps 19 cDNA in pGADT7

Smrps 19 cDNA in pGBKT7

Smatg 12 under control of met 25

promoter, URA3, $a m p^{\mathrm{R}}$

Scatg 12 under control of met 25

promoter, URA3, $a m p^{\mathrm{R}}$

egfp-Scatg8 under control of

endogenous promoter, LEU2; $a m p^{\mathrm{R}}$
This study

This study

This study

This study

This study

This study

This study

This study

This study

This study

This study

This study

This study

(Werner et al. 2016)

(Werner et al. 2016)

Thumm pers. comm., Göttingen

gpd promoter of glycerin aldehyde 3-phosphate dehydrogenase gene from A. nidulans; ccgl, promoter of the clock-controlled gene 1 from $N$. crassa; trpC promoter and terminator from the anthranilate synthase gene of A. nidulans; $a m p^{\mathrm{R}}$, ampicillin resistance; $k a n^{\mathrm{R}}$, kanamycin resistance; $h p h$, hygromycin B phosphotransferase gene; $h y g^{\mathrm{R}}$, hygromycin resistance; $n a t$, nourseothricin $\mathrm{N}$-acetyl transferase gene; $n a t^{\mathrm{R}}$, noursethricin resistance; $\mathrm{cm}^{\mathrm{R}}$, chloramphenicol resistance; $a d h l^{\mathrm{P}}, S$. cerevisiae adhl promoter; aa, amino acids.

\subsubsection{Primers}

Table 4: Overview of the used oligonucleotides.

\begin{tabular}{lll}
\hline Oligo & Sequence 5‘-3‘ & Binding position \\
\hline \multicolumn{3}{c}{ localization or multiple usage ATG12 } \\
06998_f & ATGGCATCCCCACAACCCCC & Smatg12 ORF bp 1 to 20 \\
06998_r & TCAGGTCCAGAGCAAGTAGT & $\begin{array}{l}\text { Smatg 12 3'region bp 825 } \\
\text { to 805 }\end{array}$
\end{tabular}




\begin{tabular}{|c|c|c|}
\hline Atg12-gfp-5f & $\begin{array}{l}\text { GTAACGCCAGGGTTTTCCCAGTCACGACG } \\
\text { ACATACAGCCCAATTGATGG }\end{array}$ & $\begin{array}{l}\text { Smatg } 125 \text { 'region bp - } \\
999 \text { to }-979,29-b p \\
\text { overhang to pRS426 }\end{array}$ \\
\hline Atg12-gfp-5r & $\begin{array}{l}\text { GTGAACAGCTCCTCGCCCTTGCTCACCAT } \\
\text { TTTGGGAAATTTAATGAGTG }\end{array}$ & $\begin{array}{l}\text { Smatg } 125 \text { 'region bp }-1 \\
\text { to }-20,29 \text {-bp overhang to } \\
\text { egfp }\end{array}$ \\
\hline Atg12-gfp-3f2 & $\begin{array}{l}\text { TCACTCTCGGCATGGACGAGCTGTACAAG } \\
\text { GCATCCCCACAАCCCCСАTT }\end{array}$ & $\begin{array}{l}\text { Smatg } 12 \text { ORF bp } 4 \text { to } 24, \\
29 \text {-bp overhang to } e g f p\end{array}$ \\
\hline Atg12-gfp-3r & $\begin{array}{l}\text { GCGGATAACAATTTCACACAGGAAACAGC } \\
\text { CGGTGCTTTCGCCATGGTCG }\end{array}$ & $\begin{array}{l}\text { Smatg12 3'region bp } \\
1585 \text { to } 1565,29-b p \\
\text { overhang to pRS426 }\end{array}$ \\
\hline \multicolumn{3}{|c|}{ yeast complementation ATG12 } \\
\hline Atg12_Cf & ACTAGTATGGCATCCCCACAACCCCC & $\begin{array}{l}\text { Smatg12 cDNA bp } 1 \text { to } \\
\text { 20, SpeI-restriction site }\end{array}$ \\
\hline Atg12_Cr & GTCGACTTACCCAAAAGACGGGGTCA & $\begin{array}{l}\text { Smatg } 12 \text { cDNA bp } 480 \text { to } \\
461, \text { SalI-restriction site }\end{array}$ \\
\hline Scatg12-f & 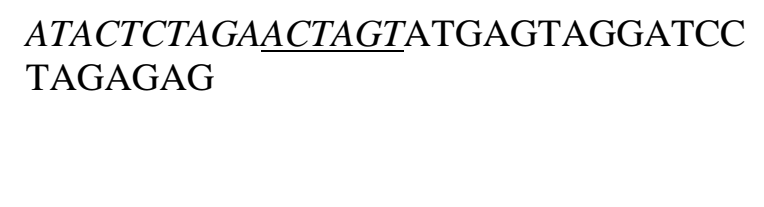 & $\begin{array}{l}\text { Scatg } 12 \text { cDNA bp } 1 \text { to } \\
20,16 \text {-bp overhang to } \\
\text { pRSmet } 25 \text { including } \\
\text { SpeI-restriction site }\end{array}$ \\
\hline Scatg12-r & $\begin{array}{l}\text { GCTTGATATCGAATTCTTAACCAAACGCT } \\
\text { ACGGATG }\end{array}$ & $\begin{array}{l}\text { Scatg } 12 \text { cDNA bp } 561 \text { to } \\
541,16 \text {-bp overhang to } \\
\text { pRSmet } 25 \text { including } \\
\text { EcoRI-restriction site }\end{array}$ \\
\hline \multicolumn{3}{|c|}{ localization, overexpression or multiple usage NBR1 } \\
\hline GFP-f & ATGGTGAGCAAGGGCGAGGAGC & egfp ORF bp 1 to 22 \\
\hline GFP-r & CTTGTACAGCTCGTCCATGCCGAGAGTG & egfp ORF bp 717 to 689 \\
\hline Dsred-f & ATGGCCTCCTCCGAGGACGT & Dsred ORF bp 1 to 20 \\
\hline Dsred-r & CAGGAACAGGTGGTGGCGGC & Dsred ORF bp 674 to 654 \\
\hline Nbr1ORF-f & ATGGCGGCACCTCCACCAAG & Smnbrl ORF bp 1 to 20 \\
\hline Nbr1-5f & 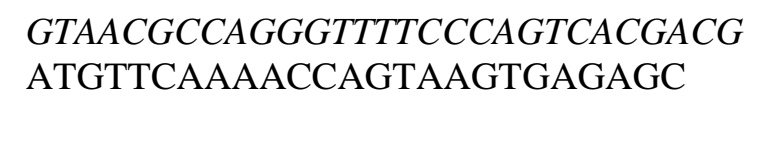 & $\begin{array}{l}\text { Smnbrl 5'region bp -992 } \\
\text { to }-968,29 \text {-bp overhang } \\
\text { to pRS426 }\end{array}$ \\
\hline GFPNbr1r & $\begin{array}{l}\text { GTGAACAGCTCCTCGCCCTTGCTCACCAT } \\
\text { CTTCCTGGAGGCGCTAACAGACTC }\end{array}$ & $\begin{array}{l}\text { Smnbrl ORF bp } 2680 \text { to } \\
2655,29 \text {-bp overhang to } \\
\text { egfp }\end{array}$ \\
\hline
\end{tabular}




$\begin{array}{ll}\text { DsredNbr1r } & \text { TCCTTGATGACGTCCTCGGAGGAGGCCAT } \\ & \text { CTTCCTGGAGGCGCTAACAGACTC } \\ \text { pRS426GPDf2 } & \begin{array}{l}\text { GTAACGCCAGGGTTTTCCCAGTCACGACG } \\ \text { GTACAGTGACCGGTGACTCT }\end{array} \\ \text { pRS426GFPrev } & \begin{array}{l}\text { GCGGATAACAATTTCACACAGGAAACAGC } \\ \text { TCGAGTGGAGATGTGGAGTG }\end{array}\end{array}$

Nbr1-1-418gfp-r

Nbr1-419-863f

Nbr1-LIR-gfp-3r

Nbr1P-r

Nbr1-T843A-f

Nbr1-Y847A-

L850A-r

Nbr1-SAcr-f1

Nbr1-SAcr-f2

Hsnbr1-f

dsred-Hsnbr1-r

pRSccg1

Pccg1-r
GTGAACAGCTCCTCGCCCTTGCTCACCAT TGACTCCATACAAGTCCCGG

ATCCATCTTCAACCATTCAGTCCGGCATT ATGGACGTAAGGACCCGTCT

GTGAACAGCTCCTCGCCCTTGCTCACCAT AGTCATGTAGGACTCGGACT

AATGCCGGACTGAATGGTTG

GAGTCCTACATGgctGATGA

GGCGTCagcGATGTCagcCTCTTC

GACCATgctCAAGTGGTCTTCCCTAAGCT CGACAAGGAGgctCCCTCTGCTgctGTCCA $\mathrm{T}$

ATTGACCATgctCAAGTGGTC

ATCCATCTTCAACCATTCAGTCCGGCATT ATGGAACCACAGGTTACTCT

TCCTTGATGACGTCCTCGGAGGAGGCCAT GAACCAGGAGAATGCTTCAC

GTAACGCCAGGGTTTTCCCAGTCACGACG TAGAAGGAGCAGTCCATCTG

TTTGGTTGATGTGAGGGGTT
Smnbrl ORF bp 2680 to 2655, 29-bp overhang to Dsred

gpd promoter bp 1 to 20 , 29-bp overhang to pRS426

$\operatorname{trp} C$ terminator bp 768 to $749,29-b p$ overhang to pRS426

Smnbrl ORF bp 1345 to 1325,29 -bp overhang to egfp

Smnbrl ORF bp 1346 to 1366, 29-bp overhang to Smnbr1 5'

Smnbrl ORF bp 2620 to 2600,29 -bp overhang to egfp

Smnbrl 5'region bp -1 to $-20$

Smnbrl ORF bp 2607 to 2627

Smnbrl ORF bp 2647 to 2623

Smnbrl ORF bp 2462 to 2522

Smnbrl ORF bp 2459 to 2480

Hsnbrl ORF bp 1 to 20, 29-bp overhang to Smnbrl 5'region

Hsnbrl ORF bp 2810 to 2790, 29-bp overhang to Dsred

ccg 1 promotor bp 1 to 20 , 29-bp overhang to pRS426

ccg1 promotor bp 921 to 901 


\begin{tabular}{|c|c|c|}
\hline Atg8-YN-f & $\begin{array}{l}\text { ACAACGTCTATATCATGGCCTAAACTAGT } \\
\text { AGATCCAAGTTTAAGGACGA }\end{array}$ & $\begin{array}{l}\text { Smatg8 ORF bp } 4 \text { to } 24 \text {, } \\
\text { 29-bp overhang to eyfp } \\
\text { (n), SpeI-restriction site }\end{array}$ \\
\hline Atg8-YN-r & $\begin{array}{l}\text { TTTCAGTAACGTTAAGTGGATCCACTAGTT } \\
\text { TACGCAGTCTCGAAATCGC }\end{array}$ & $\begin{array}{l}\text { Smatg8 ORF bp } 499 \text { to } \\
479,29-\text {-bp overhang to } \\
\text { terminator trpC, SpeI- } \\
\text { restriction site }\end{array}$ \\
\hline Nbr1-YC-f & $\begin{array}{l}C A G A C A T C A C C A T G G A G C T C A G C G G C C G \\
\underline{C A T G G C G G C A C C T C C A C C A A G}\end{array}$ & $\begin{array}{l}\text { Smnbrl ORF bp } 1 \text { to } 20 \text {, } \\
29 \text {-bp overhang to } \\
\text { promoter } g p d, \text { NotI- } \\
\text { restriction site }\end{array}$ \\
\hline Nbr1-YC-r & $\begin{array}{l}C G G G A T C T T G C A G G C C G G G C G G C G G C C \\
\underline{G C C T T C C T G G A G G C G C T A A C A G}\end{array}$ & $\begin{array}{l}\text { Smnbrl ORF bp } 2680 \text { to } \\
2660,29 \text {-bp overhang to } \\
\text { eyfp (c), NotI-restriction } \\
\text { site }\end{array}$ \\
\hline
\end{tabular}

\begin{tabular}{|c|c|c|}
\hline \multicolumn{3}{|c|}{ localization ribosomal and peroxisomal proteins } \\
\hline Smpex14-5f & $\begin{array}{l}\text { GTAACGCCAGGGTTTTCCCAGTCACGACG } \\
\text { TCGCGTAATTGTCGTGATGA }\end{array}$ & $\begin{array}{l}\text { Smpex } 145^{\prime} \text { region bp - } 860 \\
\text { to }-840,29 \text {-bp overhang } \\
\text { to pRS426 }\end{array}$ \\
\hline GFPSmpex14-r & $\begin{array}{l}\text { TCCTCGCCCTTGCTCACCATAGCGGCCTC } \\
\text { GTCGCCTCCGTTGCTGCTGG }\end{array}$ & $\begin{array}{l}\text { Smpex } 14 \text { ORF bp } 1250 \text { to } \\
\text { 1230, 29-bp overhang to } \\
\text { egfp }\end{array}$ \\
\hline hex $1-5 f$ & $\begin{array}{l}\text { GTAACGCCAGGGTTTTCCCAGTCACGACG } \\
\text { AAACCAGTTCAGCTGGTAGA }\end{array}$ & $\begin{array}{l}\operatorname{trpC} \text { promoter bp }-840 \text { to } \\
-820,29 \text {-bp overhang to } \\
\text { pRS } 426\end{array}$ \\
\hline hex1-5r & $\begin{array}{l}\text { TCCTTGATGACGTCCTCGGAGGAGGCCAT } \\
\text { TTTGGAAGATGTGTTGTTAT }\end{array}$ & $\begin{array}{l}\operatorname{trp} C \text { promoter bp - } 1 \text { to - } \\
\text { 20, 29-bp overhang to } \\
\text { Dsred }\end{array}$ \\
\hline hex 1-f & $\begin{array}{l}\text { GCGCCGAGGGCCGCCACCACCTGTTCCT } \\
\text { GGGCTACTACGACGAGGACTG }\end{array}$ & $\begin{array}{l}\text { Smhexl ORF bp } 4 \text { to } 24, \\
\text { 29-bp overhang to Dsred }\end{array}$ \\
\hline hex1-3r & $\begin{array}{l}\text { GCGGATAACAATTTCACACAGGAAACAGC } \\
\text { CAATCCTTTGTTTTTCTCCC }\end{array}$ & $\begin{array}{l}\text { Smhexl 3'region bp } 1552 \\
\text { to } 1532,29 \text {-bp overhang } \\
\text { to pRS426 }\end{array}$ \\
\hline Smrps19-5f & $\begin{array}{l}\text { GTAACGCCAGGGTTTTCCCAGTCACGAC } \\
\text { GCTGGAGATCTCCGGAGAGAC }\end{array}$ & $\begin{array}{l}\text { Smrps 19 5'region bp -721 } \\
\text { to }-701,29 \text {-bp overhang to } \\
\text { pRS426 }\end{array}$ \\
\hline Smrps19-gfp-3r & $\begin{array}{l}\text { GTGAACAGCTCCTCGCCCTTGCTCACCAT } \\
\text { CTCGTCATCCTCCTCCTCAT }\end{array}$ & $\begin{array}{l}\text { Smrps } 19 \text { ORF bp } 862 \text { to } \\
842,29 \text {-bp overhang to } \\
\text { egfp }\end{array}$ \\
\hline Smrpl25-5f & $\begin{array}{l}\text { GTAACGCCAGGGTTTTCCCAGTCACGAC } \\
\text { GAGACTTGCTTCCTGATACTT }\end{array}$ & $\begin{array}{l}\text { Smrpl25 5'region bp - } \\
1000 \text { to }-920,29-b p \\
\text { overhang to pRS426 }\end{array}$ \\
\hline
\end{tabular}




$\begin{array}{ll}\text { Smrpl25-gfp-r } & \text { GGTGAACAGCTCCTCGCCCTTGCTCACC } \\ & \text { ATGACAAGGCCGAGCTTGGTG }\end{array}$

Smrpl25-gfp-r ATGACAAGGCCGAGCTTGGTG
Smrpl25 ORF bp 686 to 666,29 -bp overhang to egfp

\begin{tabular}{|c|c|c|}
\hline \multicolumn{3}{|c|}{ knockouts and verification } \\
\hline Smnbr1-ko-5f & $\begin{array}{l}\text { GTAACGCCAGGGTTTTCCCAGTCACGACG } \\
\text { ATGTTCAAAACCAGTAAGTGAGAGC }\end{array}$ & $\begin{array}{l}\text { Smnbrl 5'region bp - } 992 \\
\text { to }-968,29 \text {-bp overhang } \\
\text { to pRS426 }\end{array}$ \\
\hline Smnbr1-ko-5r & $\begin{array}{l}\text { CCAAAAATGCTCCTTCAATATCAGTTAAC } \\
\text { AATGCCGGACTGAATGGTTGAAGAT }\end{array}$ & $\begin{array}{l}\text { Smnbrl 5'region bp -1 to } \\
-25,29 \text {-bp overhang to } \\
h p h\end{array}$ \\
\hline Smnbr1-ko-3f & $\begin{array}{l}\text { GAGTAGATGCCGACCGGGAACCAGTTAAC } \\
\text { TAGTAGTGATGCTTTGCGAACCTTG }\end{array}$ & $\begin{array}{l}\text { Smnbrl 3'region bp } 2681 \\
\text { to } 2706,29 \text {-bp overhang } \\
\text { to } h p h\end{array}$ \\
\hline Smnbr1-ko-3r & $\begin{array}{l}\text { GCGGATAACAATTTCACACAGGAAACAGC } \\
\text { AATATTTCACGAGGATGTGTTGCGA }\end{array}$ & $\begin{array}{l}\text { Smnbrl 3'region bp } 3407 \\
\text { to } 3382 \text {, 29-bp overhang } \\
\text { to pRS426 }\end{array}$ \\
\hline Smnbr1-v5f & GGTAATGATGTGTAACATTACAGAC & $\begin{array}{l}\text { Smnbrl } 5^{\prime} \text { region bp - } \\
1059 \text { to }-1034\end{array}$ \\
\hline Smnbr1-v3r & CCATGAGCGGTATCTTTCTTGGTGG & $\begin{array}{l}\text { Smnbrl 3' region bp } 780 \\
\text { to } 755\end{array}$ \\
\hline Smnbr1-vORF3-f & ATATTGTGGTCAAGAAGCCC & $\begin{array}{l}\text { Smnbrl ORF bp } 2332 \text { to } \\
2351\end{array}$ \\
\hline Smnbr1-vORF5-r & TAAGCTTCTGCTTCGCCTTG & $\begin{array}{l}\text { Smnbrl ORF bp } 360 \text { to } \\
341\end{array}$ \\
\hline $\operatorname{Atg} 12 \_5 f$ & $\begin{array}{l}\text { GTAACGCCAGGGTTTTCCCAGTCACGACG } \\
\text { ACATACAGCCCAATTGATGG }\end{array}$ & $\begin{array}{l}\text { Smatg } 125^{\prime} \text { region bp - } \\
1000 \text { to }-980,29-b p \\
\text { overhang to pRS426 }\end{array}$ \\
\hline Atg12_5r & $\begin{array}{l}\text { CAAAAAATGCTCCTTCAATATCAGTTAAC } \\
\text { TTTGGGAAATTTAATGAGTG }\end{array}$ & $\begin{array}{l}\text { Smatg } 125^{\prime} \text { region bp }-1 \text { to } \\
-20,29 \text {-bp overhang to } \\
h p h\end{array}$ \\
\hline $\operatorname{Atg} 12 \_3 f$ & $\begin{array}{l}\text { GAGTAGATGCCGACCGGGAACCAGTTAAC } \\
\text { TGCCGGGAGTCGGACAGCGT }\end{array}$ & $\begin{array}{l}\text { Smatg12 3'region bp } 334 \\
\text { to } 353,29 \text {-bp overhang to } \\
h p h\end{array}$ \\
\hline Atg12_3r & $\begin{array}{l}\text { GCGGATAACAATTTCACACAGGAAACAGC } \\
\text { GAGTGCTGTTGATGTCGATG }\end{array}$ & $\begin{array}{l}\text { Smatg12 3'region bp } \\
1033 \text { to } 1013,29-b p \\
\text { overhang to pRS426 }\end{array}$ \\
\hline Atg12_3D2 & CCGTGCTGCTCACTTTGACC & $\begin{array}{l}\text { Smatg12 ORF bp } 269 \text { to } \\
288\end{array}$ \\
\hline
\end{tabular}




\begin{tabular}{|c|c|c|}
\hline Atg12_5D1 & GTCGATTGTCGACATGGCCT & $\begin{array}{l}\text { Smatg } 125^{\prime} \text { region bp } \\
-1988 \text { to }-1969\end{array}$ \\
\hline Atg12_3D1 & AGCCTGGCCATGGTTGCCCT & $\begin{array}{l}\text { Smatg } 12 \text { 3'region bp } \\
2259 \text { to } 2278\end{array}$ \\
\hline Atg12_PRf & TGTGCAGGCTCGCTAATAAC & $\begin{array}{l}\text { Smatg } 125 \text { region bp }-699 \\
\text { to }-678\end{array}$ \\
\hline Atg12_PRr & AAAGTACGGGCGTAATCACG & $\begin{array}{l}\text { Smatg } 125 \text { region bp }-400 \\
\text { to }-419\end{array}$ \\
\hline hph-f & GTTAACTGATATTGAAGGAGCATTTTTGG & hph 5'region bp 1 to 29 \\
\hline hph-r & GTTAACTGGTTCCCGGTCGGCATCTACTC & hph ORF bp 1414 to 1386 \\
\hline $\mathrm{tC} 1$ & CACCGCCTGGACGACTAAACC & $\operatorname{trp} C$ promoter bp 95 to 75 \\
\hline $\mathrm{h} 3$ & GTACTCGCCGATAGTGGAAAC & hph ORF bp 970 to 990 \\
\hline \multicolumn{3}{|c|}{ yeast-two hybrid } \\
\hline Nbr1-t1 & $\begin{array}{l}\text { GGAGGCCAGTGAATTC } \\
\text { ATGGCGGCACCTCCACCAAGCCCT }\end{array}$ & $\begin{array}{l}\text { Smnbrl cDNA bp } 1 \text { to } 24, \\
\text { 16-bp overhang to } \\
\text { pGADT7, EcoRI- } \\
\text { restriction site }\end{array}$ \\
\hline Nbr1-t2 & $\begin{array}{l}C A C C C G G G T G G A A T T C \\
\text { TACTTCCTGGAGGCGCTAACAGA }\end{array}$ & $\begin{array}{l}\text { Smnbrl cDNA bp } 2680 \text { to } \\
\text { 2657, 16-bp overhang to } \\
\text { pGADT7, EcoRI- } \\
\text { restriction site }\end{array}$ \\
\hline ADNbr1-(HR)-f & 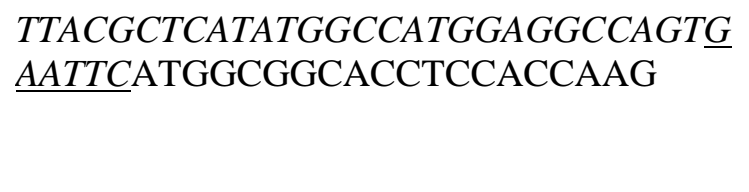 & $\begin{array}{l}\text { Smnbrl cDNA bp } 1 \text { to } 20 \text {, } \\
\text { 34-bp overhang to } \\
\text { pGADT7, EcoRI- } \\
\text { restriction site }\end{array}$ \\
\hline ADNbr1-(HR)-r & $\begin{array}{l}C C G T A T C G A T G C C C A C C C G G G T G G A A T T C \\
\text { TTACTTCCTGGAGGCGCTAA }\end{array}$ & $\begin{array}{l}\text { Smnbrl cDNA bp } 2680 \text { to } \\
2660,29 \text {-bp overhang to } \\
\text { pGADT7, EcoRI- } \\
\text { restriction site }\end{array}$ \\
\hline $\begin{array}{l}\text { ADNbr1-LIR(HR)- } \\
\mathrm{r}\end{array}$ & $\begin{array}{l}\text { TGGATCCCGTATCGATGCCCACCCGGGTG } \\
\text { GAATTCTTAAGTCATGTAGGACTCGGA }\end{array}$ & $\begin{array}{l}\text { Smnbrl cDNA bp } 2531 \text { to } \\
2510,35 \text {-bp overhang to } \\
\text { pGADT7, EcoRI- } \\
\text { restriction site }\end{array}$ \\
\hline $\begin{array}{l}\text { ADNbr1-1- } \\
418(\mathrm{HR})-\mathrm{r}\end{array}$ & 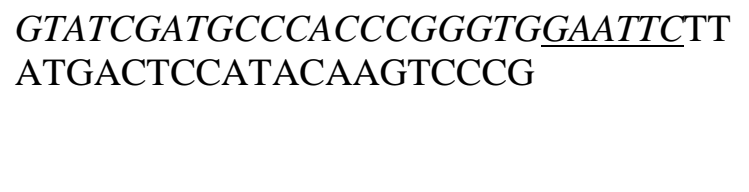 & $\begin{array}{l}\text { Smnbrl cDNA bp } 1253 \text { to } \\
\text { 1232, 27-bp overhang to } \\
\text { pGADT7, EcoRI- } \\
\text { restriction site }\end{array}$ \\
\hline $\begin{array}{l}\text { ADNbr1-419- } \\
863(\mathrm{HR})-\mathrm{f}\end{array}$ & 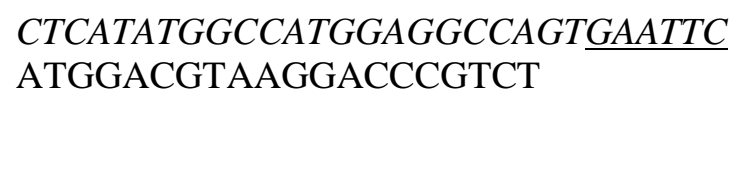 & $\begin{array}{l}\text { Smnbrl cDNA bp } 1254 \text { to } \\
1274,29 \text {-bp overhang to } \\
\text { pGADT7, EcoRI- } \\
\text { restriction site }\end{array}$ \\
\hline
\end{tabular}


Atg12_Hf

Atg12_Hr

Atg12_Hf2

Atg12_Hr2

Atg3_Hf

Atg3_Hr

Atg7_Hf

Atg7_Hr

AD-rpl25-f

AD-rpl25-r

BD-rpl25-f

BD-rpl25-r

Smrps19-c5

Smrps19-c3
GAATTCCATGGCATCCCCACAACCCCC

GTCGACGCTTACCCAAAAGACGGGGTCA

CATATGGCATCCCCACAACCCCCATT

GAATTCGCTTACCCAAAAGACGGGGTCA

CATATGAACTTCCTTAGATCAACCGC

GAATTCGCTTAGACACCCATGGTGAAGT

ACTAGTGAATTCATGGACCTCAAGTTTGC GACATTC

GTCGACAAGCTTTCAAATCAATTCTCCCT CGCCTTC

CTCATATGGCCATGGAGGCCAGT $\underline{G A A T T C}$ ATGGCATCCGAGGTGATGCA

ACCTGCATATGGCCATGGAGGCCGAATTC ATGGCATCCGAGGTGATGCA

ACCTGCATATGGCCATGGAGGCC $\underline{G A A T T C}$ ATGGCATCCGAGGTGATGCA

TTATGCGGCCGCTGCAGGTCGACGGATCC GACAAGGCCGAGCTTGGTGG
Smatg 12 cDNA bp 1 to 20, EcoRI-restriction site

Smatg 12 cDNA bp 480 to 458, NdeI-restriction site

Smatg 12 cDNA bp 4 to 24, NdeI-restriction site

Smatg 12 cDNA bp 480 to 458, EcoRI-restriction site

Smatg3 cDNA bp 1 to 20 , NdeI-restriction site

Smatg3 cDNA bp 1041 to 1022, Eco RI-restriction site

Smatg 7 cDNA bp 1 to 24 , EcoRI-restriction site

Smatg7 bp 2100 to 2076, HindIII-restriction site

Smrpl25 cDNA bp 1 to 20, EcoRI-restriction site, 29-bp overhang to pGADT7 vector

Smrpl25 cDNA bp 554 to 534, EcoRI-restriction site, 29-bp overhang to pGADT7 vector

Smrpl 25 cDNA bp 1 to 20, EcoRI-restriction site, 29-bp overhang to pGBKT7 vector

Smrpl25 cDNA bp 554 to 534, EcoRI-restriction site, 29-bp overhang to pGBKT7 vector

CCCGAATTCATGCCTGGTGGTACCACTGT Smrps 19 cDNA bp 1 to TCGG 24, EcoRI-restriction site

CCCCTGCAGTTACTCGTCATCCTCCTCCTC ATC
Smrps 19 cDNA bp 470 to 446, PstI-restriction site 
Smrps19-c3- $\quad$ CCCGGATCCTTACTCGTCATCCTCCTCCTC Smrps 19 cDNA bp 470 BamHI ATC to 446, BamHI-restriction site

Overhangs are labeled in italics. Restriction sides are underlined. Mutations are written in lowercase letters. 


\subsubsection{Chemicals and materials}

Acetic acid (Roth $\mathrm{GmbH}, 3738.2$ ), acetonitrile (Roth $\mathrm{GmbH}, 8825)$, acrylamide (Rotiphorese ${ }^{\circledR}$ Gel 40 37,5:1) (Roth GmbH, 3029.1), adenine (Sigma-Aldrich, 01830-50G), agar-agar (Roth GmbH, 5210.2), agar-agar SERVA high gel-strength (SERVA, 11396.03), agarose (Biozym Scientific GmbH, 840004), albumin bovine (Sigma-Aldrich, A9647-50G), 3-amino-1,2,4triazole (3-AT) (Sigma-Aldrich, A8056-100G), ammonium bicarbonate (Sigma-Aldrich, A6141), ammonium chloride (VWR International, BDH0208-500G), ammonium iron (II) sulfate (Roth GmbH, 203505-5G), ammonium sulfate (AppliChem, A1032,1000), ampicillin (Sigma-Aldrich, A9518-25G), arginine (AppliChem, A3709,0250), ammonium persulfate (APS) (Roth GmbH, 9592.3), bacto-yeast-extract (Oxoid LTD, LP0021), bio malt maize extract (Brau-Partner Kling, 115), biotin (Sigma-Aldrich, B4501-1G), boric acid (Roth GmbH, 6943.1), bromophenol blue (AppliChem, A3640,0005), C18 Solid phase extraction Disk (Neuss, 2215, 3M), calcium chloride (Roth GmbH, CN92.1), calcium chloride dihydrate (Roth $\mathrm{GmbH}$, 5239.1), calcofluor white (Sigma-Aldrich, 18909) carbonic anhydrase from bovine erythrocytes (Sigma-Aldrich, C3934-100MG), chloramphenicol (Sigma-Aldrich, C0378), chloroform (Merck Millipore, 1024451000), citric acid-monohydrate (Sigma-Aldrich, C7129), 7-amino-4-chloromethylcoumarin (CMAC) (Invitrogen, C-2110), Coomassie brilliant blue R250 (Roth GmbH, 3862.1), Coomassie brilliant blue G250 (Roth GmbH, 9598.1), CSM-AdeHis-Leu-Trp-Ura (MP Biomedicals, 4550-122), 4',6-Diamidine-2'-phenylindole (DAPI) (AppliChem, A1001), dialysis filter (Merck Millipore, VSWP01300), 2’-deoxy-nucleotids$5^{\prime}$ triphosphate (dNTPs) (Thermo Scientific, R0191), Difco ${ }^{\mathrm{TM}}$ skim milk (BD Biosciences, 232100), deoxyribonucleic acid single stranded from salmon testes (ss-DNA) (Sigma Aldrich, D1626-1G), Difco ${ }^{\mathrm{TM}}$ yeast nitrogen base w/o amino acids and ammonium sulfate (BD Biosciences, 233520), disodium hydrogen phosphate (Merck-Millipore, 1065855000), DMF (dimethylformamide) (Roth GmbH, T921.1), dimethyl sulfoxide (DMSO) (Merck Millipore, 1029310500), 1,4-Dithiothreitol (DTT) (AppliChem, A1101,0025), EDTA-free protease inhibitor cocktail (Roche, 04693132001), ethylenediamine tetraaceticacid disodium salt dihydrate (EDTA) (Roth GmbH, 8043.2), electroporation cuvettes (VWR International, 7321137), ethanol (VWR International, 20821.321), ethidium bromide (Sigma-Aldrich, 46065), "Flat Optical 8-Cap Strip" $0.2 \mathrm{ml}$ (Biozym, 712100), formaldehyde (Roth GmbH, 4979.2), formamide (Sigma-Aldrich, 47670), formic acid (Merck Millipore, 1002641000), FM4-64 (Invitrogen, F34653), GeneRuler 1 kb DNA ladder (Thermo Scientific, SM0313), “GeneScreen Hybridization Transfer Membrane" (PerkinElmer Lifesciences, NEF988001PK), GFP-Trap agarose beads (Chromotek, gta-10), glass beads $\varnothing$ 0.25-0.5 mm (Roth GmbH, A553.1), glass beads $\varnothing$ 2.85-3.45 mm (Roth GmbH, A557.1), glucose (AppliChem, A3617,1000), glycine (Roth GmbH, 0079.1), glycerol (VWR International, 24388.295), HEPES (4-(2-hydroxyethyl)- 
1-piperazineethanesulfonicacid) (Roth GmbH, 9105.4), histidine (Merck Millipore, 1.04351.0025), hydrochloric acid (Roth $\mathrm{GmbH}, 4625.2)$, hydrogen peroxide $30 \%\left(\mathrm{H}_{2} \mathrm{O}_{2}\right)$ (Merck Millipore, 8.22287.2500), hygromycin B (Merck Millipore, 400051-10MU), high performance chemiluminescence film (Amersham Hyperfilm ${ }^{\mathrm{TM}}$ ECL, 28906837), iodoacetamide (Sigma-Aldrich, I1149), isopropyl- $\beta$-D-galactopyranoside (IPTG) (Roth $\mathrm{GmbH}, 2316.3$ ), iron (II) chloride (Roth $\mathrm{GmbH}, 231-753-5)$, iron (II) sulfate heptahydrate (Sigma-Aldrich, 31236), imidazole (Roth GmbH, X998.1), isopropanol (AppliChem, A0900,2500GL), kanamycin sulfate (Sigma-Aldrich, 60615), leucine (AppliChem, A1426,0100), lithium acetate (Roth GmbH, 5447.1), maize flour (Mühle Levers, Bochum, Germany; Mühle Udo Kellner, Schimberg, Germany), magnesium chloride hexahydrate (Merck Millipore, 1.05833.1000), magnesium sulfate heptahydrate (Roth GmbH, P027.2), manganese (II) chloride tetrahydrate (Roth GmbH, T881.1), manganese (II) sulfate monohydrate (Roth GmbH, 4487.1), methanol (VWR International, 20864.320), nitrocellulose blotting membrane (GE Healthcare, 10600003), Nonidet ${ }^{\circledR}$ P40 (NP-40) (AppliChem, 9016-459), nourseothricin (WernerBioAgents, 5004000), nylon membrane (GE Healthcare, RPN203B), PagerulerTM Prestained Protein Ladder (Thermo Fisher, SM0671), PEG 4000 (AppliChem, A1249), PEG 6000 (Sigma-Aldrich, 81255), phenol (AppliChem, A1153,0500), phosphoric acid (Roth GmbH, 6366.1), PMFS (phenylmethylsulfonyl) fluoride (Sigma-Aldrich, P-7626), ponceau S (Sigma Aldrich, P3504), potassium acetate (Merck Millipore, 1.04820.1000), potassium chloride (AppliChem, A3582), potassium dihydrogen phosphate (Merck Millipore, 1.04873.1000), potassium hydroxide (Roth $\mathrm{GmbH}, 6751.1$ ), potassium nitrate (Merck Millipore, 1.05063.1000), sodium dodecyl sulfate (SDS) (Roth GmbH, 4360.2), sodium acetate (Roth GmbH, 6773.2), sodium chloride (AppliChem, A3597,1000), sodium dihydrogen phosphate monohydrate (Merck Millipore, 1.06346.1000), sodium hydroxide (VWR International, 28244.295), sodium molybdate-dihydrate (Sigma-Aldrich, 31439), sorbitol (Roth $\mathrm{GmbH}, 6213.1$ ), $\beta$-mercaptoethanol (Roth $\mathrm{GmbH}, 4227.1$ ), sterile filter 0.45/0.2 $\mu \mathrm{m}$ (Sarstedt, 83.1826/83.1826.001), sucrose (AppliChem, A4734,1000), TEMED (N,N,N',N'tetramethylethylenediamine) (Roth $\mathrm{GmbH}, 2367.3)$, Tris (tris-hydroxymethyl-aminomethane) (Roth GmbH, AE15.2), trichloracetic acid (TCA) (Sigma-Aldrich, 91230), tris/HCl (Roth GmbH, 9090.3), Trizol (Invitrogen, 15596026), tryptone/peptone (Roth GmbH, 8952,2), tryptophan (MP Biomedicals, 4061-012), Tween 20 ${ }^{\circledR}$ (AppliChem, A4974), uracil (MP Biomedicals, 4061-212), urea (Roth GmbH, 2317.3), whatman paper B002 580x600 mm (Schleicher \& Schuell, 88-3852), xylene cyanol (AppliChem, A4976,0005), yeast extract (Roth $\mathrm{GmbH}, 2904.1$ ), zinc chloride (Sigma-Aldrich, 14424), zinc sulfate heptahydrate (Roth GmbH, K301.1). 


\subsubsection{Kits}

AlkPhos Direct Labelling and Detection Kit (Amersham, GE Healthcare, RPN3680), Immobilon $^{\mathrm{TM}}$ Western HRP Substrate (Merck Millipore, WBKLS0100), FastGene plasmid Mini Kit (Nippon Genetics Co., Ltd, Lot. 15048), Qiagen PCR Cloning Kit (Qiagen, 231124), QIAprep Spin Miniprep Kit (Qiagen, 27106), QIAquick Gel Extraction Kit (Qiagen, 28704), QIAquick PCR Purification Kit (Qiagen, 28104), Transcriptor High Fidelity cDNA Synthesis Kit (Roche, 05091284001), ZR fungal/Bacterial DNA mini prep (Zymoresearch, D6005).

\subsubsection{Enzymes}

Calf Intestine Alkaline Phosphatase (CIAP) (Thermo Scientific, EF0341), DNase I (Thermo Scientific, EN0521), lambda phosphatase (New England Biolabs GmbH, P0753), lysozyme (Serva, 28262.03), MolTaq DNA polymerase (Molzym, P-010-1000), natuzym (Schliessmann, 5090), Phusion ${ }^{\circledR}$ Hot Start High-Fidelity DNA polymerase (Thermo Scientific, F-549S), restriction endonucleases (Thermo Scientific), RNase A (Roth GmbH, 7164.1), phosphatase inhibitor cocktail tablets (Roche, 04693132001), T4 DNA ligase (Thermo Scientific, EL0011), trypsin digestion buffer (SERVA Electrophoresis, 37283.01).

\subsubsection{Buffers and solutions}

\section{Antibiotics/antimycotics}

Ampicillin stock solution:

Kanamycin stock solution:

chloramphenicol:

hygromycin:

nourseothricin dihydrogen sulfate: $50 \mu \mathrm{g} / \mathrm{ml}$

\section{Transformation and supplementation of $S$. macrospora}

Protoplasting buffer (PPP):

Transformation buffer (TPS):

Topagar:
$13 \mathrm{mM} \mathrm{Na}_{2} \mathrm{HPO}_{4}, 45 \mathrm{mM} \mathrm{KH} \mathrm{PO}_{4}, 0.6 \mathrm{M} \mathrm{KCl}, \mathrm{pH} 6.0$

$1 \mathrm{M}$ sorbitol, $80 \mathrm{mM} \mathrm{CaCl}_{2}, \mathrm{pH} 7.4$

TPS with $25 \%$ PEG (PEG6000)

$0.8 \mathrm{M} \mathrm{NaCl}, 0.8 \%$ agar-agar, $0.025 \%$ hygromycin $\mathrm{B}$ $(110 \mathrm{U} / \mathrm{ml}), 0.025 \%$ nourseothricin dihydrogen sulfate $(50 \mu \mathrm{g} / \mathrm{ml})$

$5 \%(\mathrm{w} / \mathrm{v})$ citric acid $\left(\mathrm{C}_{6} \mathrm{H}_{8} \mathrm{O}_{7}\right.$ monohydrate $)$,

$5 \%(\mathrm{w} / \mathrm{v}) \mathrm{ZnSO}_{4}$ heptahydrate,

$1 \%(\mathrm{w} / \mathrm{v}) \mathrm{Fe}\left(\mathrm{NH}_{4}\right)_{2}\left(\mathrm{SO}_{4}\right)_{2}$ hexahydrate, 
$0.25 \%(\mathrm{w} / \mathrm{v}) \mathrm{CuSO}_{4}$ pentahydrate,

$0.05 \%$ (w/v) $\mathrm{MnSO}_{4}$ monohydrate,

$0.05 \%(\mathrm{w} / \mathrm{v}) \mathrm{H}_{3} \mathrm{BO}_{3}$,

$0.05 \%(\mathrm{w} / \mathrm{v}) \mathrm{Na}_{2} \mathrm{MoO}_{4}$ dihydrate

Biotin stock solution: $\quad 0.01 \%(\mathrm{w} / \mathrm{v})$ biotin, $50 \%(\mathrm{v} / \mathrm{v})$ ethanol

Arginine stock solution: $\quad 100 \mathrm{mg} / \mathrm{ml}$ in A. dest.

S. macrospora lysis buffer (gDNA): $10 \mathrm{mM}$ Tris/HCl (pH 8.0), 1 mM EDTA, $100 \mathrm{mM} \mathrm{NaCl}$,

$2 \% \operatorname{SDS}$

Preparation agar: $\quad 5 \%(w / v)$ agar-agar in A. dest.

Transformation and supplementation of $S$. cerevisiae

Lithium acetate $(10 \mathrm{x})$ :

TE (10x)

$\operatorname{LiAcTE}(\mathrm{D})(1 \mathrm{x})$ :

DTT:

Sorbitol:

LiAcTE(D) + PEG:

Salmon sperm (ss) DNA:
$1 \mathrm{M}$ lithium acetate, $\mathrm{pH} 7.5$

$100 \mathrm{mM}$ Tris (pH 8.0), 10 mM EDTA

$1 \mathrm{ml}$ lithium acetate (10x), $1 \mathrm{ml}$ TE (10x), A. dest.

1M DTT in $\mathrm{H}_{2} \mathrm{O}$

$1 \mathrm{M}$ sorbitol in $\mathrm{H}_{2} \mathrm{O}$

$50 \%$ (w/v) PEG 4000, in 1x (v/v) LiAcTE(D)

$1 \%(\mathrm{w} / \mathrm{v}) \mathrm{ssDNA}$

\section{Amino acid stock solutions (yeast)}

Adenine stock solution:

Leucine stock solution:

Histidine stock solution:

Uracil stock solution:

Tryptophan stock solution:

\section{DNA methods}

dNTP mix (10 mM):

EtBr stock solution:

DNA loading dye (6x):

TBE (10x):

Agarose gel:
$0.02 \%(\mathrm{w} / \mathrm{v})$ adenine in A. dest., final conc. $0.002 \%$

$1 \%(\mathrm{w} / \mathrm{v})$ leucine in A. dest., final conc. $0.003 \%$

$1 \%(\mathrm{w} / \mathrm{v})$ histidine in A. dest., final conc. $0.002 \%$

$0.02 \%(\mathrm{w} / \mathrm{v})$ uracil in A. dest., final conc. $0.002 \%$

$0.02 \%$ (w/v) tryptophan in A. dest., final conc. $0.002 \%$

10 mM dATP, dCTP, dGTP, dTTP each in A. dest.

$10 \mathrm{mg} / \mathrm{ml}$ ethidium bromide in A. dest.

$0.25 \%(\mathrm{w} / \mathrm{v})$ xylene cyanol,

$0.25 \%(\mathrm{w} / \mathrm{v})$ bromophenol blue,

$40 \%(\mathrm{w} / \mathrm{v})$ sucrose

$1 \mathrm{M}$ Tris/HCl, $1 \mathrm{M}$ boric acid, 20 mM EDTA, pH 8.3

$1 \%(\mathrm{w} / \mathrm{v})$ agarose in $1 \mathrm{x}$ TBE 


\section{Plasmid isolation according to Birnboim and Doly (1979)}

BD solution I:

BD solution II:

BD solution III:

\section{Protein methods}

5x SDS-PAGE loading dye:

TBST(10x):

Skim milk blocking solution:

Ponceau staining solution:

SDS-PAGE-running buffer:

Lysis buffer (S. macrospora, protein):

Dilution buffer:

Lysis buffer (S. cerevisiae):

fixing solution

Coomassie staining solution:

SDS solution:

Transfer buffer:

Buffer b:
$50 \mathrm{mM}$ glucose, $10 \mathrm{mM}$ EDTA, $25 \mathrm{mM}$ Tris/ $\mathrm{HCl}, 0.2 \%$ lysozyme

$0.4 \mathrm{M} \mathrm{NaOH}, 2 \%(\mathrm{w} / \mathrm{v})$ SDS (mixed 1:1)

$3 \mathrm{M}$ potassium acetate, $1.8 \mathrm{M}$ formic acid

125 mM Tris/HCl (pH 6.8), $50 \%$ (v/v) glycerol, $2 \%$ (w/v) SDS, $0.01 \%$ (w/v) bromophenol blue, $0.01 \%(\mathrm{w} / \mathrm{v}) \beta$-mercaptoethanol $100 \mathrm{mM}$ Tris/ $\mathrm{HCl}$ (pH 7.5), $1.5 \mathrm{M} \mathrm{NaCl}$, $0.1 \%(\mathrm{v} / \mathrm{v})$ Tween $20^{\circledR}$ (add fresh) $5 \%(\mathrm{w} / \mathrm{v})$ Difco $^{\mathrm{TM}}$ skim milk in 1xTBST $0.2 \%$ Ponceau S, $3 \%$ TCA $1.5 \%(\mathrm{w} / \mathrm{v})$ Tris ( $\mathrm{pH} 8.3), 9.4 \%$ (w/v) glycine, $20 \%(w / v)$ SDS

$10 \mathrm{mM}$ Tris (pH 7.5), $150 \mathrm{mM} \mathrm{NaCl}, 0.5 \mathrm{mM}$ EDTA (pH 8.0), 2 mM DTT, 1 mM PMSF, 1x protease inhibitor cocktail, $10 \%$ glycerol, $0.5 \%$ NP-40 $10 \mathrm{mM}$ Tris (pH 7.5), $150 \mathrm{mM} \mathrm{NaCl}, 0.5$ mM EDTA (pH 8.0), $1 \mathrm{mM}$ PMSF, 1x protease inhibitor cocktail 1.85 $\mathrm{M} \mathrm{NaOH}, 7.5 \%$ (v/v) $\beta$-mercaptoethanol $45 \%$ ethanol

$10 \%$ acetic acid

$0.02 \%(\mathrm{w} / \mathrm{v})$ Coomassie brilliant blue R250, $0.02 \%(\mathrm{w} / \mathrm{v})$ Coomassie brilliant blue G250, $42.5 \%(\mathrm{v} / \mathrm{v})$ ethanol $0.5 \%(\mathrm{v} / \mathrm{v})$ methanol $10 \%(\mathrm{v} / \mathrm{v})$ acetic acid $10 \%(\mathrm{w} / \mathrm{v})$ SDS in A. dest. $192 \mathrm{mM}$ glycine, $25 \mathrm{mM}$ Tris, $20 \%$ (v/v) methanol, $0.02 \%$ SDS 100 mM Tris/HCl (pH 7.5), 200 mM NaCl, 20 \% glycerol, $5 \mathrm{mM}$ EDTA 
Buffer b+:

buffer $\mathrm{b}+1 \mathrm{x}$ protease inhibitor cocktail,

$0.5 \% \beta$-mercaptoethanol

PXG (pellet X-Gal) assay

Phosphate buffered saline

1.4 $\mathrm{M} \mathrm{NaCl}, 27 \mathrm{mM} \mathrm{KCl}, 100 \mathrm{mM} \mathrm{Na} 2 \mathrm{HPO}_{4}, 17.6 \mathrm{mM}$

(PBS, 10x):

$\mathrm{KH}_{2} \mathrm{PO}_{4}, \mathrm{pH} 7.4$

X-Gal:

$2 \%(\mathrm{w} / \mathrm{v})$ in DMF

\section{Southern blot solutions}

Buffer I:

$0.25 \mathrm{M} \mathrm{HCl}$

Buffer II:

$0.5 \mathrm{M} \mathrm{NaOH}, 1.5 \mathrm{M} \mathrm{NaCl}$

Buffer III:

$1.5 \mathrm{M} \mathrm{NaCl}, 0.5 \mathrm{M}$ Tris

First washing buffer:

$2 \mathrm{M}$ Urea, $0.1 \%$ (w/v) SDS, $50 \mathrm{mM} \mathrm{NaH}_{2} \mathrm{PO}_{4} \times \mathrm{H}_{2} \mathrm{O}$

$(\mathrm{pH} 7.0)$,

$150 \mathrm{mM} \mathrm{NaCl}, 1 \mathrm{mM} \mathrm{MgCl} 2,0.2 \%$ blocking reagent

Second washing buffer (20x): $\quad 1 \mathrm{M}$ Tris, $2 \mathrm{M} \mathrm{NaCl}, 1 \mathrm{mM} \mathrm{MgCl}_{2}, \mathrm{pH} 10.0$

Prehybridisation buffer:

$0.5 \mathrm{M} \mathrm{NaCl}$ with $4 \%$ "blocking reagent"

\subsubsection{Cultivation media}

$\underline{\text { E. coli }}$

LB (Luria-Bertani): $1 \%(\mathrm{w} / \mathrm{v})$ tryptone, $0.5 \%$ yeast extract, $0.5 \% \mathrm{NaCl}, \mathrm{pH} 7.2 ; 1.5 \%(\mathrm{w} / \mathrm{v})$ agar-agar for solid medium; addition of ampicillin $(100 \mu \mathrm{g} / \mathrm{ml})$, kanamycin $(50 \mu \mathrm{g} / \mathrm{ml})$ or chloramphenicol $(50 \mu \mathrm{g} / \mathrm{ml})$ for selection.

SOB (super optimal broth): $2 \%(\mathrm{w} / \mathrm{v})$ tryptone, $0.5 \%(\mathrm{w} / \mathrm{v})$ yeast extract, $10 \mathrm{mM} \mathrm{NaCl}$, $2.5 \mathrm{mM} \mathrm{KCl}, 10 \mathrm{mM} \mathrm{MgCl}, 10 \mathrm{mM} \mathrm{MgSO}_{4}, \mathrm{H}_{2} \mathrm{O}, \mathrm{pH} 7.0$

TB: $10 \mathrm{mM}$ HEPES, $15 \mathrm{mM} \mathrm{CaCl}_{2}$ x 2 $\mathrm{H}_{2} \mathrm{O}, 250 \mathrm{mM} \mathrm{KCl}, 55 \mathrm{mM} \mathrm{MnCl}_{2}$ x 4H $2 \mathrm{O}, \mathrm{pH} 6.7$

\section{$\underline{\text { S. cerevisiae }}$}

YEPD (yeast extract, peptone, dextrose): $2 \%(\mathrm{w} / \mathrm{v})$ tryptone, $2 \%(\mathrm{w} / \mathrm{v})$ glucose, $1 \%(\mathrm{w} / \mathrm{v})$ yeast extract, $\mathrm{pH} 5.8 ; 1.5 \%(\mathrm{w} / \mathrm{v})$ agar-agar for solid medium.

SD (synthetic defined): $0.17 \%(\mathrm{w} / \mathrm{v})$ Difco $^{\text {тм }}$ yeast nitrogen base w/o amino-acids and ammonium sulfate, $2 \%(\mathrm{w} / \mathrm{v})$ glucose, $0.5 \%(\mathrm{w} / \mathrm{v})$ ammonium sulfate, $0.064 \%(\mathrm{w} / \mathrm{v})$ CSMAde-His-LeuTrp-Ura (0.002 \% L-methionine, $0.005 \%$ L-arginine hydrochloride, L-isoleucine, Llysine hydrochloride, L-phenylalanine, L-tyrosine each, $0.008 \%$ L-aspartic acid, $0.01 \%$ and L-threonine, $0.014 \%$ L-valine), $\mathrm{pH} 5.8 ; 2 \%$ (w/v) agar-agar SERVA for solid medium. Selection of transformants was performed by exclusion of respective amino acid(s). 


\section{$\underline{\text { S. macrospora }}$}

BMM (biomalt maize medium): $0.8 \%(\mathrm{w} / \mathrm{v})$ bio malt maize extract and maize flour $(25 \mathrm{~g} / \mathrm{L})$, $\mathrm{pH} 6.5 ; 1.5 \%(\mathrm{w} / \mathrm{v})$ agar-agar for solid medium; addition of hygromycin B $(110 \mathrm{U} / \mathrm{ml})$ or nourseothricin dihydrogen sulfate $(50 \mu \mathrm{g} / \mathrm{ml})$ for selection.

BMM sodium acetate: $\mathrm{BMM}+0.5 \%(\mathrm{w} / \mathrm{v})$ sodium acetate (sporulation induction); $1.5 \%$ (w/v) agar-agar for solid-medium.

CMS (complex media, sucrose): $1 \%(\mathrm{w} / \mathrm{v})$ glucose, $0.2 \%(\mathrm{w} / \mathrm{v})$ tryptone/peptone, $0.2 \%(\mathrm{w} / \mathrm{v})$ yeast extract, $0.15 \%$ (w/v) $\mathrm{KH}_{2} \mathrm{PO}_{4}, 0.05 \%$ (w/v) $\mathrm{KCl}, 0.05 \%$ (w/v) $\mathrm{MgSO}_{4}$ heptahydrate, $0.37 \%(\mathrm{w} / \mathrm{v}) \mathrm{NH}_{4} \mathrm{Cl}, 10.8 \%(\mathrm{w} / \mathrm{v})$ sucrose, $0.01 \%(\mathrm{v} / \mathrm{v})$ trace-element stock solution $(10 \mathrm{mg} / \mathrm{l}$ $\mathrm{ZnSO}_{4}, 10 \mathrm{mg} / \mathrm{Fe}(\mathrm{II}) \mathrm{Cl}_{2}, 10 \mathrm{mg} / \mathrm{l} \mathrm{MnCl}_{2}$ ), $\mathrm{pH}$ 6.5; $1.5 \%$ (w/v) agar-agar for solid medium.

SWG (Sordaria Westergaards medium): 1x Westergaard's $\left(0.1 \%\right.$ (w/v) $\mathrm{KNO}_{3}, 0.1 \%$ (w/v) $\mathrm{KH}_{2} \mathrm{PO}_{4}, 0.05 \%$ (w/v) $\mathrm{MgSO}_{4}$ heptahydrate, $0.01 \%$ (w/v) $\mathrm{NaCl}, 0.01 \%$ (w/v) $\mathrm{CaCl}_{2},[0.01 \%$ $(\mathrm{v} / \mathrm{v})$ trace-element stock solution $+0.1 \%(\mathrm{v} / \mathrm{v})$ chloroform] $), 2 \%(\mathrm{w} / \mathrm{v})$ glucose, $0.1 \%(\mathrm{w} / \mathrm{v})$ arginine, $0.1 \%(\mathrm{v} / \mathrm{v})$ biotin stock solution, $\mathrm{pH} 6.5 ; 1.5 \%(\mathrm{w} / \mathrm{v})$ agar-agar for solid medium; addition of hygromycin $\mathrm{B}(110 \mathrm{U} / \mathrm{ml})$ or nourseothricin dihydrogen sulfate $(50 \mu \mathrm{g} / \mathrm{ml})$ for selection.

SWG $0.5 \%$ G+OA: SWG but glucose is reduced to $0.5 \%, 0.15 \%(\mathrm{w} / \mathrm{v})$ oleic acid, $0.05 \%$ (w/v) NP-40.

SWG-G+OA: SWG without glucose, $0.15 \%$ oleic acid, $0.05 \%$ NP-40. 


\subsection{Methods}

\subsubsection{Cultivation of organisms}

\section{$\underline{\text { E. coli }}$}

Cultivation of E. coli strains was done at $37^{\circ} \mathrm{C}$ on solid LB medium or in liquid LB medium while shaking at $180 \mathrm{rpm}$. Selection was carried out by the addition of $100 \mu \mathrm{g} / \mathrm{ml}$ ampicillin, $50 \mu \mathrm{g} / \mathrm{ml}$ kanamycin or $50 \mu \mathrm{g} / \mathrm{ml}$ chloramphenicol.

\section{$\underline{\text { S. cerevisiae }}$}

Yeast strains were inoculated on solid YEPD medium (complete medium) and solid SDmedium (minimal medium) supplemented with appropriate amino acids and incubated at $30^{\circ} \mathrm{C}$. Liquid cultures were incubated in corresponding liquid medium while shaking at $100 \mathrm{rpm}$.

\section{$\underline{\text { S. macrospora }}$}

Cultivation of $S$. macrospora strains was conducted on solid corn meal medium (BMM), complex rich medium containing $10.8 \%$ sucrose (CMS) and fruiting-body development inducing SWG medium at $27^{\circ} \mathrm{C}$ with continuous light. For phenotypic analysis under different stress conditions the temperature was changed or SWG medium was supplemented with 2.5 mM 3-amino-1,2,4-triazole (3-AT), 0.5 M sorbitol, $0.25 \mathrm{M} \mathrm{NaCl}, 0.01 \%, 0.02 \%, 0.05 \% \mathrm{H}_{2} \mathrm{O}_{2}$ or $0.005 \%$ SDS, respectively. To induce nitrogen starvation SWG was modified by omitting $\mathrm{KNO}_{3}$, arginine and biotin or in combination. Liquid cultures were grown in corresponding liquid medium at $27^{\circ} \mathrm{C}$ in petri dishes. For analysis of the growth velocity, $30-\mathrm{cm}$ race tubes were filled with $25 \mathrm{ml}$ of solid SWG medium or stress-inducing medium and inoculated with a mycelia plug of $0.5 \mathrm{~cm}$ in diameter at one end (Nolting and Pöggeler 2006). The growth front was marked every $24 \mathrm{~h}$ for five consecutive days of three replications in triplets. The growth rate was calculated in $\mathrm{cm}$ per day. All quantitative analyses of $S$. macrospora strains were done using SWG medium and were analyzed statistically as described in 2.2.6.3. For phenotypic analysis and light microscopy $S$. macrospora strains were grown on cellophane or glass slides covered with solid SWG or BMM medium at $27^{\circ} \mathrm{C}$ with continuous light. The growth period differed between 3-10 d depending on the developmental stage. To induce sexual development in liquid media, S. macrospora was grown in liquid BMM medium at $27^{\circ} \mathrm{C}$ in floating cultures. To prevent sexual development in liquid medium $S$. macrospora was cultivated in Erlenmeyer flasks with $100 \mathrm{ml}$ of liquid BMM medium shaken at $130 \mathrm{rpm}$ at $27^{\circ} \mathrm{C}$. 


\subsubsection{Preparation and transformation of competent microorganisms}

\section{$\underline{\text { E. coli }}$}

Chemically competent E. coli MACH1 cells (Invitrogen (Carlsbad, USA)) were prepared by inoculation of a few colonies in $50 \mathrm{ml} \mathrm{SOB}$ in a $250 \mathrm{ml}$ Erlenmeyer flask while shaking overnight at $20^{\circ} \mathrm{C}$ and $50 \mathrm{rpm}$. After incubation, and when the optical density of $600 \mathrm{~nm}\left(\mathrm{OD}_{600}\right)$ had reached 0.6 , the cells were kept on ice for $10 \mathrm{~min}$ and harvested by centrifugation at 3000 $\mathrm{rpm}$ at $4{ }^{\circ} \mathrm{C}$ for $10 \mathrm{~min}$. The cells were resuspended in $16 \mathrm{ml} \mathrm{TB}$ and kept on ice for further 10 min, followed by centrifugation at the same conditions as the last step. The cell pellet was resuspended in $4 \mathrm{ml}$ TB plus $280 \mu \mathrm{l}$ DMSO and incubated on ice for $10 \mathrm{~min}$. The cells were aliquoted to $1.5 \mathrm{ml}$ cups and frozen in liquid nitrogen for storage at $-80^{\circ} \mathrm{C}$. For transformation of chemically competent E. coli MACH1 cells plasmid DNA was added to thawed competent cells and incubated for 30 min on ice (Sambrook and Russell 2001). After a $90 \mathrm{sec}$ heat shock at $42^{\circ} \mathrm{C}$, cells were incubated on ice for $2 \mathrm{~min}$ followed by the addition of $800 \mu 1$ liquid LB and incubation for $1 \mathrm{~h}$ at $37^{\circ} \mathrm{C}$ and $200 \mathrm{rpm}$. Cells were plated on solid LB medium with $100 \mu \mathrm{g} / \mu \mathrm{l}$ ampicillin, $50 \mu \mathrm{g} / \mu \mathrm{l}$ kanamycin or $50 \mu \mathrm{g} / \mu \mathrm{l}$ chloramphenicol.

\section{S. cerevisiae}

S. cerevisiae strain PJ69-4A was used as host for homologous recombination experiments performed according to Colot et al. (2006). For the preparation of electro-competent S. cerevisiae cells, a $50 \mathrm{ml}$ culture was grown to an $\mathrm{OD}_{600}$ of 0.6-1.2 in YEPD. The cells were subsequently pelleted and incubated with $1 \mathrm{x} \operatorname{LiAcTE}(\mathrm{D})$. After $45 \mathrm{~min}$ at $30^{\circ} \mathrm{C}$ and $100 \mathrm{rpm}$, $0.5 \mathrm{ml} 1 \mathrm{M}$ DTT were added. After additional incubation at $30^{\circ} \mathrm{C}$ and $100 \mathrm{rpm}$ for $15 \mathrm{~min}$ the cells were washed with A. dest. and $1 \mathrm{M}$ sorbitol. For the transformation $40 \mu 1$ competent cells were mixed with $500 \mathrm{ng}$ DNA and electroporated in $0.2 \mathrm{~cm}$ electroporation cuvettes in the "Eppendorf Electroporator 2510" (Eppendorf, Germany) at $1.5 \mathrm{kV}$. After electroporation, 800 $\mu \mathrm{l}$ of $1 \mathrm{M}$ sorbitol were added and $200 \mu \mathrm{l}$ portions of the yeast suspension were plated on respective solid selection medium.

Another lithium acetate transformation method was used as described by Ito et al. (1983). For this, a $10 \mathrm{ml}$ culture was grown to an $\mathrm{OD}_{600}$ of $0.6-1.2$ in YEPD at $30^{\circ} \mathrm{C}$ in a rotating wheel. Afterwards, the cultures were spun down for $4 \mathrm{~min}$ at $4000 \mathrm{rpm}$ in a $15 \mathrm{ml}$ falcon tube. The supernatant was discarded and the pellet resuspended in $4 \mathrm{ml} \mathrm{1x} \mathrm{LiAcTE(D).} \mathrm{Afterwards,} \mathrm{the}$ cells were again spun down and resuspended in approximately $360 \mu 1$ 1x LiAcTE(D), resulting in $400 \mu \mathrm{l}$ cell suspension. Each $200 \mu \mathrm{l}$ were utilized for one transformation. To each transformation sample $20 \mu \mathrm{l}$ of ssDNA, $1-3 \mu \mathrm{l}$ of the plasmid of interest and $800 \mu \mathrm{l} 50 \% 1 \mathrm{x}$ 
LiAcTE(D)+PEG were added and the transformation sample was mixed. After an incubation of $30 \mathrm{~min}$ at $30^{\circ} \mathrm{C}$ the cells were heat shocked for $25 \mathrm{~min}$ at $42^{\circ} \mathrm{C}$ followed by a centrifugation for $30 \mathrm{sec}$ at $7000 \mathrm{rpm}$. The pelleted cells were resuspended in $800 \mu$ liquid YEPD and incubated on a rotary shaker at $30^{\circ} \mathrm{C}$ for $1 \mathrm{~h}$. Finally, the cells were pelleted again, resuspended in the remaining supernatant and plated on SD medium with $2 \%$ agar lacking the specific amino acids or nucleic acids necessary for selection. The samples were then incubated at $30^{\circ} \mathrm{C}$ until growth was observed.

\section{S. macrospora}

Standard transformation protocols for S. macrospora were conducted as described previously (Pöggeler et al. 1997; Nowrousian et al. 1999). For the transformation of S. macrospora protoplasts had to be prepared. Mycelium was inoculated in liquid BMM medium with 6-8 agar plugs. After three days the mycelium was harvested and transferred into a sterile $250 \mathrm{ml}$ flask and incubated with $0.4 \mathrm{~g}$ natuzyme in $20 \mathrm{ml} \mathrm{PPP}$ buffer for $2 \mathrm{~h}$ at $27^{\circ} \mathrm{C}$ and $100 \mathrm{rpm}$. The next step was the separation of the cell debris from the protoplasts. Therefore, the protoplast solution was transferred to a filter (frit, pore size 1) to remove the debris and collect the protoplasts in falcon tubes. The solution was filled up to $50 \mathrm{ml}$ with PPP buffer and centrifuged $\left(4 \mathrm{~min}, 4^{\circ} \mathrm{C}\right.$, $4400 \mathrm{rpm}$ ). After this step the protoplast solution was washed with $10 \mathrm{ml}$ PPP buffer and subsequently centrifuged $\left(4 \mathrm{~min}, 4^{\circ} \mathrm{C}, 4400 \mathrm{rpm}\right)$. The pelleted protoplasts were resuspended in 100-200 $\mu \mathrm{l}$ TPS buffer and $20 \mu \mathrm{g}$ DNA were mixed with $100 \mu \mathrm{l}$ protoplasts and incubated for $10 \mathrm{~min}$ on ice. After the addition of $200 \mu \mathrm{l}$ PEG 6000 TPS $(0.25 \mathrm{~g} / \mathrm{ml})$ the solution was incubated for $20 \mathrm{~min}$ at RT. Finally, the mixture was plated on solid CMS medium in $150 \mu \mathrm{l}$ aliquots. On the next day the plates were covered with $9 \mathrm{ml}$ topagar containing the appropriate antibiotic. Positive primary transformants were isolated after 1-3 d.

\subsubsection{Construction of single-spore isolates of $S$. macrospora}

Heterokaryotic primary transformants that were able to form perithecia were directly used to generate homokaryotic single-spore isolates. Otherwise, the strains were crossed with the sporecolor mutant strain fus 1-1 to produce hybrid perithecia. The fus1-1 strain has lost its ability to produce melanin resulting in brown ascospores (Nowrousian et al. 2012). For this, two different $S$. macrospora strains were crossed by placing two agar pieces $\left(0.25 \mathrm{~cm}^{2}\right)$, each containing one strain, towards each other in a distance of $5 \mathrm{~cm}$ on a petri dish with solid SWG medium. After incubation for $8-10 \mathrm{~d}$ at $27^{\circ} \mathrm{C}$, the crossing front is formed in the middle of the petri dish containing the recombinant perithecia. The recombinant hybrid perithecia contain asci with 4 
brown (fus1-1 strain) and 4 black ascospores in typical 4:4 or 2:2:2:2 segregation patterns. Single-spore isolates (ssi) were done by transferring and opening the recombinant perithecia on preparation agar using a Zeiss "Stemi 2000-C" stereo microscope (Zeiss). Single spores were collected with a sterile needle and placed on solid BMM sodium acetate medium, supplemented with either hygromycin B $(110 \mathrm{U} / \mathrm{ml})$ or nourseothricin dihydrogen sulfate $(50 \mu \mathrm{g} / \mathrm{ml})$ or both for selection.

\subsubsection{Molecular biological methods}

\subsubsection{Preparation of DNA}

\subsection{Isolation of plasmid DNA from E. coli}

For the extraction of plasmid DNA from E. coli two different kits were used according to the manufacturer's manual: the QIAprep Spin Miniprep or the FastGene plasmid Mini Kit. In addition, a modified plasmid extraction protocol according to Birnboim and Doly (1979) was used. For this purpose an E. coli $5 \mathrm{ml} \mathrm{LB}$ culture with the appropriate antibiotic was grown over night at $37^{\circ} \mathrm{C}$ and $200 \mathrm{rpm}$. The cells were harvested by centrifugation at $5000 \mathrm{rpm}$ for $5 \mathrm{~min}$. The pellet was vortexed with $200 \mu \mathrm{l}$ of the BDI solution, $10 \mu \mathrm{l}$ Rase A $(10 \mathrm{mg} / \mathrm{ml})$ and 0.4 mg lysozyme. After 5 min of incubation at RT $200 \mu 1$ BDII were added to the cell suspension, mixed and stored on ice for $10 \mathrm{~min}$. Subsequently, $200 \mu \mathrm{l} \mathrm{BDIII}$ were added and the solution was mixed by gently inverting the tube followed by $10 \mathrm{~min}$ incubation on ice. The supernatant was yielded by centrifugation at $13000 \mathrm{rpm}$ for $10 \mathrm{~min}$ and transferred to a new cup. For the precipitation of the DNA $750 \mu \mathrm{l}$ ice-cold isopropanol were added and incubated at $-20^{\circ} \mathrm{C}$ for at least $20 \mathrm{~min}$. Subsequently, the DNA was harvested with the same speed for $5 \mathrm{~min}$ and washed with $500 \mu \mathrm{l}$ of ice-cold $70 \%$ ethanol. Again, the DNA was pelletized before the supernatant was discarded and the pellet was dried before dissolving in 100-200 $\mu \mathrm{l} \mathrm{A}$. dest.

\subsection{Plasmid DNA isolation from S. cerevisiae}

To isolate recombinant plasmid DNA from S. cerevisiae transformants, cells from selective media plates were removed with a sterile cotton bud and dissolved in $1 \mathrm{ml}$ sterile water. Subsequently, the cells were harvested by centrifugation and the DNA was isolated using the ZR fungal/Bacterial DNA mini prep Kit according to manufacturer's manual. 


\subsection{Isolation of genomic DNA and RNA from $S$. macrospora}

The isolation of genomic DNA from S. macrospora was achieved either by the phenol/chloroform extraction method according to Pöggeler et al. (1997) or a modified sodium acetate/isopropanol precipitation. After growth for three days in liquid BMM medium the mycelium was harvested, dried and grinded in liquid nitrogen. After the addition of $600 \mu 1$ lysis buffer mycelium powder was incubated for $30 \mathrm{~min}$ at $70^{\circ} \mathrm{C}$. Afterwards $400 \mu \mathrm{l}$ of a $3 \mathrm{M}$ sodium acetate solution were added followed by incubation for $10 \mathrm{~min}$ at $-20^{\circ} \mathrm{C}$. The mixture was centrifuged for $10 \mathrm{~min}$ at $13000 \mathrm{rpm}$ and the obtained supernatant was mixed with same volume of ice-cold isopropanol and incubated for $20 \mathrm{~min}$ at $-80^{\circ} \mathrm{C}$. After a new centrifugation $(10 \mathrm{~min}$, $4{ }^{\circ} \mathrm{C}, 7000 \mathrm{rpm}$ ) the pellet was washed with $500 \mu \mathrm{l}$ ice-cold $70 \%$ ethanol and dried under the hood. The genomic DNA was resuspended in 50-100 $\mu 1$ distilled water.

The extraction of RNA was done according to Elleuche and Pöggeler (2009). In brief, the mycelium was grinded to powder with liquid nitrogen and mixed with $1 \mathrm{ml}$ trizol. After centrifugation for $10 \mathrm{~min}$ at $13000 \mathrm{rpm}$ the supernatant was transferred to a new reaction tube and mixed with $0.2 \mathrm{ml}$ chloroform. Again, the mixture was centrifuged and $500 \mu 1$ of the newly obtained supernatant were mixed with the same volume of ice-cold isopropanol. After incubation for $10 \mathrm{~min}$ at RT the RNA was centrifuged, washed with $500 \mu \mathrm{l}$ ice-cold $70 \%$ ethanol and dried under the hood. Finally, the RNA was resuspended in $120 \mu \mathrm{l}$ water and incubated for $30 \mathrm{~min}$ at $1000 \mathrm{rpm}$ and $60^{\circ} \mathrm{C}$.

\subsubsection{Hydrolysis and ligation of nucleic acids}

Nucleic acids were hydrolyzed using specific restriction endonucleases (1 U/1 $\mu \mathrm{g}$ DNA) according to manufacturer's manual in setups of 10-50 $\mu$. The ligation of nucleic acids was carried out using the T4 DNA ligase as suggested by the manufacturer. Reactions were incubated at RT for $30 \mathrm{~min}$ to $2 \mathrm{~h}$. To avoid self-ligation plasmids were treated with "Calf Intestine Alkaline phosphatase" after hydrolysis and prior to ligation.

\subsubsection{Purification of nucleic acids}

Prior to transformation or ligation experiments salts from the PCR or hydrolysis reactions needed to be removed from the aqueous nucleic-acid solution. Therefore, dialysis filters (Merck-Millipore, Germany) were placed on the water surface within a petri dish and $15 \mu 1$ of the PCR fragments or the hydrolyzed plasmid DNA were positioned on the top. The dialysis then proceeded for $15 \mathrm{~min}$. Alternatively, the QIAquick PCR Purification Kit was used to purify DNA fragments. Furthermore, DNA could also be loaded on a $1 \%$ agarose gel and DNA 
fragments were isolated with the QIAquick Gel Extraction Kit as described by the manufacturer.

\subsubsection{Polymerase chain reaction (PCR)}

The PCR technique was used for in vitro amplification of genes or gene fragments from genomic, plasmid or complementary DNA using specific forward and reverse oligonucleotides (Mullis and Faloona 1987). The PCR reactions were performed with either the Phusion ${ }^{\circledR}$ Hot Start High-Fidelity DNA polymerase characterized by a low error rate or the MolTaq DNA polymerase for analytical and colony-PCR. The reactions were carried out in the cycler machines "Eppendorf Mastercycler epgradient S" (Eppendorf, Germany) or MyCycler (BioRad, USA) as suggested by the manufacturer's. Components used for the standard $50 \mu 1$ PCR reactions for Phusion and MolTaq polymerase were as follows:

Table 5: Composition of a $50 \mu \mathrm{l}$ PCR reaction for Phusion-or MolTaq polymerase.

\begin{tabular}{ccc}
\hline components & Phusion polymerase & MolTaq polymerase \\
\hline water & $34 \mu \mathrm{l}$ & $35.8 \mu \mathrm{l}$ \\
$5 \mathrm{x}$ reaction buffer & $10 \mu \mathrm{l}$ & $10 \mu \mathrm{l}$ \\
dNTPs $(10 \mathrm{mM})$ & $1 \mu \mathrm{l}$ & $1 \mu \mathrm{l}$ \\
primer 1 $(10 \mathrm{pM})$ & $1 \mu \mathrm{l}$ & $1 \mu \mathrm{l}$ \\
primer 2 $(10 \mathrm{pM})$ & $1 \mu \mathrm{l}$ & $1 \mu \mathrm{l}$ \\
template DNA $(50-100 \mathrm{ng})$ & $1 \mu \mathrm{l}$ & $1 \mu \mathrm{l}$ \\
DMSO & $1.5 \mu \mathrm{l}$ & - \\
polymerase & $0.5 \mu \mathrm{l}$ & $0.2 \mu \mathrm{l}$ \\
\hline
\end{tabular}

\subsubsection{Agarose gel electrophoresis of nucleic acids}

This electrophoresis method allows the horizontal separation of DNA within an agarose gel due to its size. For this purpose the samples were mixed with $6 x$ loading dye and loaded on a $1 \%$ agarose gel (1g agarose in $100 \mathrm{ml}$ 1x TBE buffer). The DNA was separated by 70-130 V using a gel chamber "Mupid one" (Biozym Scientific GmbH, Germany) filled with $0.5 \%$ TBE buffer. The GeneRuler ${ }^{\mathrm{TM}} 1 \mathrm{~kb}$ DNA Ladder was used as standard. Subsequently, the gel was stained with $0.0001 \%$ ethidium bromide solution for $15 \mathrm{~min}$ and visualized by UV transillumination. The result was documented with the „BioDocAnalyzer“ (Biometra, Germany). 


\subsubsection{Southern blot and hybridization}

Deletion of genes was verified by Southern hybridization (Southern 1975). First, 30-50 $\mu \mathrm{g}$ of gDNA was hydrolyzed in a $50 \mu 1$ reaction volume and separated on a $1 \%$ agarose gel for 90$120 \mathrm{~min}$ at $90 \mathrm{~V}$. After the run, the gel was soaked for $10 \mathrm{~min}$ in buffer I $(0.25 \mathrm{M} \mathrm{HCl}), 25 \mathrm{~min}$ in buffer II (0.5 M NaOH, $1.5 \mathrm{M} \mathrm{NaCl})$ and $30 \mathrm{~min}$ in buffer III (1.5 M NaCl, 0.5 M Tris). A semi-dry blot onto a nylon membrane was performed for $3 \mathrm{~h}$. Afterwards, the membrane was dried for $7 \mathrm{~min}$ at $70^{\circ} \mathrm{C}$ and the DNA was cross-linked via "UV-light exposure" (Biometra, Germany) of each side for $3 \mathrm{~min}$. The AlkPhos Direct Labelling and Detection Kit was used for labeling the defined probe. The hybridization of the membrane with the probe as well as the detection was performed according to the manufacturer's manual.

\subsubsection{Oligonucleotide synthesis, sequencing and sequence analysis}

The synthesis of oligonucleotide used in this study was done by Eurofins MWG Operon (Ebersberg, Germany). All primers used for this study are listed in Table 4. DNA sequencing was performed by the G2L-sequencing service of the Göttinger Genom Labor (Georg-August University of Göttingen, Germany) and Seqlab (Sequence Laboratories Göttingen GmbH, Germany). The resulting sequences were analyzed via software Chromas Lite 2.01 (1998-2005 Technelysium Pty Ltd) and compared to the plasmid sequence created in Clone Manager 7 with the use of the nucleotide BLASTN search from the public database at NCBI (http://www.ncbi.nlm.nih.gov/). Multiple protein sequence alignments were done using the ClustalX program (Thompson et al. 1997) with sequences of different organisms obtained after BLASTP search from database at NCBI and visualized using the program GeneDoc 2.7.0.0. Molecular weights and isoelectric points were calculated with programs from the ExPASy Proteomics Server (http://www.expasy.org) as well as domain analysis of the proteins. Phosphorylation sites were predicted using the online tool NetPhos3.1 (http://www.cbs.dtu.dk/services/NetPhos).

\subsubsection{Phylogenetic analysis}

Multiple protein sequence alignments were performed using the ClustalX alignment program (Thompson et al. 1997). Phylogenetic analysis was made with programs from package PHYLIP version 3.695 (http://evolution.genetics.washington.edu/phylip.html). Distance matrices were calculated using the program PRODIST and were then used for constructing phylogenetic trees with the neighbor-joining (NJ) program NEIGHBOR. To evaluate the statistical significance a bootstrap analysis with 1000 iterations of bootstrap samplings and reconstruction of trees was 
performed. A majority rule consensus tree was subsequently generated with the program CONSENSE, displayed with TreeView 1.6.6 (Page 1996) and saved for graphical representation with Adobe Illustrator (CS2).

\subsubsection{Plasmid construction using homologous recombination in yeast}

Yeast cells (PJ69-4A) can perform homologous recombination, which allows for the assembly of circular plasmids from several linear DNA fragments. One 29-bp overhang is required between two fragments or the vector backbone and is sufficient for the yeast cell to activate the DNA repair mechanism (Colot et al. 2006). The assembly of a plasmid by homologous recombination requires a hydrolyzed vector backbone and linear DNA fragments. Knockout plasmids consists of the 5' and 3'-flanking region of the gene of interest interrupted by a resistance cassette whereas plasmids for localization and complementation studies encode native or suitable promoter and terminator regions for expression in respective wanted organism, an open reading frame of the gene of interest (gDNA) and if necessary a gene for a tag. To combine the single fragments into a plasmid, some primers exhibited at least 29-bp overhang to the neighboring regions for homologous recombination reactions in S. cerevisiae. The yeast was transformed as described in 2.2.2 and resulting plasmids were isolated from yeast transformants (2.2.4.1.2) and retransformed in E. coli cells (2.2.2). The multiplied plasmid was isolated (2.2.4.1.1) and purified (2.2.4.3) for transformation into S. macrospora (2.2.2).

The same method was sometimes used to generate plasmids for the yeast-two hybrid experiments. Then the cDNA of the gene of interest was combined with the linearized pGADT7 or pGBKT7 vectors.

\subsubsection{Generation of $S$. macrospora deletion strains}

For generation of $S$. macrospora $\Delta$ Smnbr1 and $\Delta$ Smatg12 deletion strains the homologous recombination procedure in $S$. cerevisiae was used to create a plasmid containing the respective knockout construct (Colot et al. 2006) (2.2.5). The deletion constructs consisting of the $h p h$ resistance cassette flanked by the 5'- and 3'-flanking regions of the gene of interest. After homologous recombination in yeast, the plasmids were isolated and the deletion cassettes were amplified from the respective plasmids, purified and transformed into S. macrospora strain $\Delta \mathrm{ku} 70$ which is inhibited in the non-homologous end joining (Pöggeler and Kück 2006). Consequently, the deletion cassette will be integrated by gene replacement at the desired gene locus. Afterwards, the $\Delta \mathrm{ku} 70$ background was eliminated by crossing positive primary 
transformants with the spore-color mutant fus1-1 and single spores of hybrid perithecia were selected on hygromycin media (2.2.3). The constructed single-spore deletion strain was verified by PCR and Southern blot.

\subsubsection{Generation of $\Delta$ Smnbr1 strain}

To delete the Smnbrl gene in S. macrospora the following knockout construct was constructed according to Colot et al. (2006). The 5'- (1050-bp fragment) and 3'- (785-bp fragment) flanking regions of Smnbrl were amplified from wt gDNA using the primer pairs Smnbr1-ko5f/Smnbr1-ko-5r and Smnbr1-ko-3f/Smnbr1-ko-3r carrying 29-bp overhangs for the pRS426 vector and the hygromycin resistance B $(h p h)$ cassette, respectively. The $h p h$-cassette (1419-bp fragment) was amplified from plasmid pCB1003 with the primers hph-f/hph-r. Subsequently, the three amplicons were co-transformed together with the XhoI-linearized vector pRS426 into the yeast strain PJ69-4A as described in 2.2.2 and in Mayrhofer et al. (2006). Transformants were selected on synthetic defined (SD)-medium lacking uracil. The recombinant plasmid pSmnbr1-KO, consisting of the upstream and downstream sequence of Smnbrl interrupted by the $h p h$-cassette, was isolated from yeast as described in 2.2.4.1.2. Plasmid pSmnbr1-KO served as template to generate the 3254-bp knockout fragment by PCR with primer pair Smnbr1-ko-5f/Smnbr1-ko-3r. The amplicon was desalted and then transformed into S. macrospora $\Delta \mathrm{ku} 70$ strain to facilitate the Smnbrl knockout by homologous recombination (Pöggeler and Kück 2006). Hygromycin B resistant primary transformants were analyzed by PCR with primer pairs Smnbr1-v5f/tC1 (1166 bp) and h3/Smnbr1-v3r (873 bp) to verify the homologous recombination event at the desired Smnbrl gene locus. Primary transformants of S. macrospora are usually heterokaryotic carrying $\Delta \mathrm{ku} 70\left(n a t^{\mathrm{R}}\right)$ and mutant nuclei $\left(n a t^{\mathrm{R}} / h y g^{\mathrm{R}}\right)$. To obtain homokaryotic deletion mutants and to eliminate the $\Delta \mathrm{ku} 70$ (nat ${ }^{\mathrm{R}}$ ) background, primary transformants were crossed with the brown spore-color mutant fus1-1 (S23442) (Nowrousian et al. 2012). The recombinant hybrid perithecia could be easily identified because the crossing partners differed in their spore color. Black spores from hybrid perithecia were isolated and selected on BMM agar plates containing hygromycin B (110 U/ml) and $0.5 \%$ sodium acetate (2.2.3). The resulting homokaryotic deletion strain $\triangle$ Smnbrl was tested by PCR for the absence of the Smnbrl gene using primer pairs Smnbr1-v5f/Smnbr1-vORF5-r and Smnbr1-vORF3-f/Smnbr1-v3r. gDNA of S. macrospora wt strain was used as control and fragment sizes of $1425 \mathrm{bp}$ and $1133 \mathrm{bp}$ were expected. Deletion of Smnbrl was verified by Southern hybridization as described in 2.2.4.6 (Southern 1975). Therefore, 30-50 $\mu \mathrm{g}$ of gDNA was hydrolyzed with PstI (2.2.4.2). The 1050-bp probe was amplified with primer pair Smnbr1- 
ko-5f/Smnbr1-ko-5r from S. macrospora gDNA and purified with the QIAquick gel extraction kit.

\subsubsection{Construction of $\Delta$ Smatg12 strain}

The generation of the Smatg12 knockout strain was done in the same way as for the Smnbrl deletion strain by homologous replacement. To delete the Smatg12 gene in S. macrospora, a plasmid was generated containing the 5'-(1058 bp) and 3'- (700 bp) flanking regions of Smatg12 which were amplified from S. macrospora wt gDNA using the primer pairs Atg12_5f/Atg12_5r and Atg12_3f/Atg12_3r carrying 29-bp overhangs for the pRS426 vector and the hygromycin resistance B ( $h p h$ ) cassette, respectively. The $h p h$-cassette (1419 bp) was amplified from plasmid pCB1003 with the primers hph-f/hph-r. Subsequently, the three amplicons were co-transformed together with the XhoI-linearized vector pRS426 into the yeast strain PJ69-4A (Mayrhofer et al. 2006). Positive transformants containing the recombinant plasmid pSmatg12-KO were selected on SD-medium lacking uracil. It is characterized by the upstream and downstream sequence of Smatg12 interrupted by the hph-cassette. Plasmid pSmatg12-KO served as template to generate the 3235-bp knockout fragment by PCR with primer pair Atg12_5f/Atg12_3r. The amplicon was desalted and transformed into S. macrospora $\Delta \mathrm{ku} 70$ strain to facilitate the Smatg 12 knockout by homologous recombination (Pöggeler and Kück 2006). Hygromycin B resistant primary transformants were analyzed by PCR with primer pairs Atg12_3D1/h3 and tC1/Atg12_5D1 to verify the homologous recombination event at the desired Smatg12 gene locus. The heterokaryotic primary transformants of $S$. macrospora carrying $\Delta \mathrm{ku} 70\left(n a t^{\mathrm{R}}\right)$ and mutant nuclei $\left(h y g^{\mathrm{R}}\right)$. To obtain homokaryotic deletion mutants and to eliminate the $\Delta \mathrm{ku} 70\left(n a t^{\mathrm{R}}\right)$ background, positive primary transformants were crossed with the brown spore-color mutant fus1-1 (S23442) (Nowrousian et al. 2012) as described in 2.2.3 and 2.2.6.

Single spores from hybrid perithecia were picked and selected on BMM agar plates containing only hygromycin $\mathrm{B}(110 \mathrm{U} / \mathrm{ml})$ and $0.5 \%$ sodium acetate. The positive homokaryotic $\triangle$ Smatg12 strain was tested by PCR for the absence of the Smatg12 gene using primer pair Atg12_5D1/Atg12_3D2 in comparison to gDNA of S. macrospora wt strain. Additionally, deletion of Smatg12 was verified by Southern hybridization (Southern 1975). For this, 30 - $50 \mu \mathrm{g}$ of $\Delta$ Smatg12 gDNA was hydrolyzed with $B g l I$. The 300 -bp probe was amplified with primer pair Atg12_PRf/Atg12_PRr from S. macrospora gDNA and purified with QIAquick gel extraction kit. 


\subsubsection{Morphological investigation of S. macrospora deletion strains}

The morphological investigation of perithecia and the mycelium growth during the sexual cycle of S. macrospora was done with the "Digital Microscope VHX-500F" (Keyence, Germany) or the "AxioImager M1 microscope" (Zeiss, Germany). For this purpose, S. macrospora strains were either plated on solid SWG or BMM medium for macroscopic investigations or grown on SWG medium with cellophane or glass slides covered with solid SWG medium at $27^{\circ} \mathrm{C}$ with continuous light for microscopic investigations (Bloemendal et al. 2012). The growth period differed between 3-10 d depending on the developmental stage. The ascus rosettes were visualized by cracking the perithecia on object slides into a drop of water, the outer perithecia tissue was removed and asci were covered with a cover slide to evoke ascus rosette formation. For examination of the foraging abilities of $S$. macrospora strains, a plug test was performed according to Josefsen et al. (2012). An agar plug with a diameter of $0.5 \mathrm{~cm}$ was put into a cellculture plate $\left(6\right.$ well, $17.2 \mathrm{ml}$ ) and incubated for five days in a damp chamber at $27^{\circ} \mathrm{C}$. All quantitative analysis of S. macrospora strains were done using SWG medium. Counting of perithecia per $\mathrm{cm}^{2}$ was performed after $8 \mathrm{~d}$ (wt, $\Delta$ Smnbr1::Smnbr1-egfp $\left.{ }^{\text {ect }}\right)$ or $10 \mathrm{~d}(\Delta \mathrm{Smnbr} 1)$ of growth with the "Digital Microscope VHX-500F" (Keyence, Germany) from nine independent measurements of three independent experiments $(n=27)$. Discharged ascospores were analyzed after $10 \mathrm{~d}$ of growth by washing off the discharged ascospores from the lid of the petri dish, and counting the spores using a thoma cell counter chamber (W. Schreck, Hofheim). The asci in an ascus rosette were also investigated by microscopic observations after $8 \mathrm{~d}$ or $10 \mathrm{~d}$. The analysis of the ascospores and asci were statistically analyzed from 25 independent measurements of four independent experiments $(n=100)$. For phenotypic analysis under stress-inducing conditions SWG medium was modified as described in 2.2.1. The SWG medium was inoculated with a mycelia plug of $0.5 \mathrm{~cm}$ in diameter in the middle of a petri dish $(\varnothing 9 \mathrm{~cm})$ and after the indicated days investigated by microscopic and macroscopic observations. The phenotypic investigations were done in three biological replicates. 


\subsubsection{Plasmid construction and localization analysis of fluorescence-tagged S. macrospora genes}

Most of the fluorescence-tagged plasmids were constructed by homologous recombination as described in 2.2.5. These plasmids were used for localization studies as well as to complement the knockout strains by transformation of the plasmids.

\subsubsection{Plasmid construction for Smatg12, Smatg8 and Smnbr1 fluorescence- tagged versions}

For localization of SmATG12 an egfp-tagged variant of Smatg 12 under the control of the native promoter and terminator was constructed. The Smatg12 promoter region was amplified from wt gDNA using primer pair Atg12-gfp-5f/Atg12-gfp-5r (1058-bp fragment) and the coding region including the 3' region of Smatg12 (1615-bp fragment) with primer pair Atg12-gfp3f2/Atg12-gfp-3r. The egfp was amplified from plasmid p1783-1 using primer pair GFP-f/GFPr (688-bp fragment). Some fragments exhibit overhangs to the neighbored regions. All fragments were subcloned into the XhoI-linearized vector pRSnat by homologous recombination in yeast and resulted in an N-terminally egfp-tagged version of Smatg12 in plasmid pegfp-Smatg12.

To localize the full-length SmNBR1 a C-terminal EGFP-tagged and DsRED-tagged variant of Smnbrl under control of the native promoter and $\operatorname{trpC}$ terminator of A. nidulans was constructed. The Smnbrl promoter together with the coding region was amplified from wt gDNA using primer pair Nbr1-5f/GFPNbr1r (3731-bp fragment) or Nbr1-5f/DsredNbr1r (3731-bp fragment). The egfp (1540-bp fragment) or Dsred (1497-bp fragment), both flanked with the $\operatorname{trpC}$ terminator at the 3'end, were amplified from plasmids p1783-1 and pRHN1with primer pairs GFP-f/pRS426GFPrev and Dsred-f/pRS426GFPrev, respectively. Some of the primers exhibited at least 29-bp overhangs to the neighboring regions for homologous recombination reactions in S. cerevisiae (Colot et al. 2006). All fragments were subcloned into the XhoI-linearized vector pRSnat and resulted in C-terminal EGFP- or DsRED-tagged versions of SmNBR1 in plasmid pSmnbr1-egfp and pSmnbr1-Dsred, respectively (Table 3). To analyze different truncated variants of SmNBR1 the following plasmids were constructed according to the full-length version, which differ just in the coding region achieved by using specific primers and pSmnbr1-egfp as template DNA. The promoter and coding region for plasmid pSmnbr1-1418-egfp, encodes the N-terminal region, was amplified using Nbr1-5f/Nbr1-1-418gfp-r (2395bp fragment). For plasmid pSmnbr1-419-863-egfp, encodes the C-terminal region, the primer 
pair Nbr1-419-863f/GFPNbr1r (1384-bp fragment) was used for amplification of the ORF and the Smnbrl promoter was amplified with primer pair Nbr1-5f/Nbr1P-r (1020-bp fragment). The version of Smnbrl without the LIR motif (pSmnbr1-1-843-egfp) was amplified using Nbr1-5f/ Nbr1-LIR-gfp-3r (3661-bp fragment). All mutated versions of Smnbrl were also under control of the native promoter and the following primers were used to introduce the different mutations by PCR: Nbr1-Y847A-L850A-r, Nbr1-T843A-f and Nbr1-SAcr-f1. For overexpression plasmid pccg1-Smnbr1-egfp, the constitutive promoter ccgl from $N$. crassa was amplified from plasmid pHAN1 with primer pair pRSccg1/Pccg1-r (950 bp) and connected to the Smnbrl coding region and $e g f p$ with the $\operatorname{trp} C$ terminator.

For analyzing SmATG8 and SmNBR1 interaction in S. macrospora via Bimolecular Fluorescence Complementation (BiFC) assays recombinant plasmids pRS-YN (hyg) pRS-YC (nat) were generated encoding the $\mathrm{N}$ - or $\mathrm{C}$-terminus of the yellow fluorescence protein (YFP$\mathrm{N}$ or YFP-C) under the control of the constitutive $g p d$ promoter and the $\operatorname{trpC}$ terminator of A. nidulans. To amplify $y f p-n$ or $y f p-c$ the vectors $\mathrm{pEYFP-N}$ and $\mathrm{pEYFP-C}$ were used as templates with primer pair pRS426GPDf2/pRS426GFPrev. The fragments of 2155 bp (yfp-n) and 1978 bp $(y f p-c)$ were introduced in the XhoI-hydrolyzed pRShyg and pRSnat vector by homologous recombination in yeast resulting in vectors $\mathrm{pRS}-\mathrm{YN}$ and $\mathrm{pRS}-\mathrm{YC}$, respectively. The Smatg8 coding sequence was amplified with primer pair Atg8-YN-f/Atg8-YN-r from gDNA (558-bp fragment) and fused it after $y f p-n$ in the SpeI-hydrolyzed vector pRS-YN (hyg) by ligation. The gDNA sequence of the coding region of Smnbrl was amplified with primers Nbr1-YC-f/Nbr1-YC-r (2738-bp fragment) and fused in front of $y f p-c$ in the NotI-hydrolyzed vector pRS-YC (nat). Both plasmids were introduced in S. macrospora wild type strain.

\subsubsection{Plasmid construction of DsRED-tagged $H$. sapiens NBR1 protein}

To express the H. sapiens nbrl gene in the S. macrospora $\Delta$ Smnbr1 strain, plasmid pHsnbr1Dsred was constructed expressing Dsred C-terminal of Hsnbrl gene under the control of the the S. macrospora Smnbrl promoter. First, the Hsnbrl cDNA was amplified from plasmid pOTB7 using primer pair Hsnbr1-f/dsred-Hsnbr1-r carrying 29-bp overhangs either to the Smnbrl promoter region or the Dsred (2868-bp fragment). The Smnbrl promoter region was amplified from gDNA with primer pair Nbr1-5f/Nbr1P-r and resulted in a 1020-bp fragment with 29-bp overhang to the pRSnat vector. The Dsred flanked with the $\operatorname{trpC}$ terminator at the 3'end (1497-bp fragment), was amplified from plasmid pRHN1 with primer pair Dsredf/pRS426GFPrev. All fragments were subcloned into the XhoI-linearized vector pRSnat by 
homologous recombination in yeast. Subsequently, the $\Delta$ Smnbr1 strain was transformed with the plasmid pHsnbr1-Dsred (2.2.2) and analyzed for complementation ability.

\subsubsection{Plasmid construction of EGFP-tagged ribosomal proteins}

To analyze ribosomal proteins in vivo SmRPL25 and SmRPS19 were C-terminal tagged with EGFP in the same way as described in 2.2.7.1. The native promoter region and ORF of the ribosomal subunit genes Smrpl25 and Smrps19 with 29-bp overhangs were amplified from S. macrospora wt gDNA with the primer combination Smrpl25-5f/Smrpl25-gfp-r (1734-bp fragment) and Smrps19-5f/Smrps19-gfp-3r (1641-bp fragment). The egfp together with trpC terminator from A. nidulans was amplified from plasmid p1783-1 using primer pair GFPf/pRS426GFPrev (1540-bp fragment). By homologous recombination in yeast the respective amplicons were cloned into the XhoI-linearized pRSnat vector and resulted in plasmids pSmrpl25-egfp and pSmrps19-egfp.

\subsubsection{Plasmid construction of EGFP-tagged peroxisomal proteins}

Plasmid pSmpex14-egfp was constructed by homologous recombination in yeast as described in 2.2.7.1. pSmpex 14-egfp encodes the peroxisomal membrane protein SmPEX14 C-terminally tagged with EGFP. The fusion gene is under the control of the native promoter and the trpC terminator of A. nidulans. The Smpex14 ORF together with its native promoter region was amplified using primers Smpex14-5f/GFPSmpex14-r with gDNA as template, which resulted in a 2102-bp fragment with 29-bp overhangs. The egfp fragment together with the $\operatorname{trpC}$ terminator (1540-bp fragment) was amplified using primer pair GFP-f/pRS426GFPrev and plasmid p1783-1 as template.

To generate a plasmid that expresses the prominent Woronin body protein SmHEX1 Nterminally tagged with DsRED under the control of the $\operatorname{trp} C$ promoter plasmid pDsred-Smhex 1 was generated. The $\operatorname{trp} C$ promoter region was amplified using primer pair hex $1-5 \mathrm{f} / \mathrm{h}$ ex $1-5 \mathrm{r}$ and plasmid p1783-1 as template which resulted in a fragment of 898 bp including 29-bp overhangs. Using pRHN1 as template, the Dsred gene was amplified with primers Dsred-f/Dsred-r. The amplicon has a size of $674 \mathrm{bp}$. The coding and $3^{\prime}$-flanking region of Smhexl was amplified from $S$. macrospora gDNA with primer pair hex1-f/hex-3r and results in a fragment of $1610 \mathrm{bp}$ incuding 29-bp overhangs.

All fragments were combined in the XhoI-linearized pRSnat vector by homologous recombination in yeast. 


\subsubsection{Light and fluorescence microscopic investigations}

For light and fluorescence microscopic analysis, S. macrospora strains were grown on solid medium on top of a piece of cellophane $(2 \times 2 \mathrm{~cm})$ in petri dishes at $27^{\circ} \mathrm{C}$ for the indicated hours. Subsequently, the cellophane sheet with the mycelium was put on an object slide, covered with water and a cover slip for microscopic analysis. Microscopic investigations were carried out using an "AxioImager M1 microscope" (Zeiss, Jena, Germany) and images were captured with a "Photometrix CoolSNAP HQ camera" (Roper Scientific, Photometrics, Tucson, USA) and processed with the programs MetaMorph (version 6.3.1; Universal Imaging), ImageJ (Image Processing and Analysis in Java) and Adobe Illustrator (CS2). For the detection of fluorescence the "X-cite 120 PC lamp" (EXFO) was utilized with different filter sets. The “chroma filter set 49002" (exciter ET470/40, emitter ET525/50 and beam splitter T4951pxr) was used to visualize EGFP fluorescence, detection of DsRED was achieved with the "chroma filter set 49005" (exciter ET545/30, emitter ET620/60, beam splitter T5701p) and the "chroma filter set 49003" (exciter ET500/20, emitter ET535/30 and beam splitter T515lp) was used for the detection of YFP. Staining of vacuolar membranes was achieved by applying 50-100 $\mu$ of a FM4-64 solution (Invitrogen, F34653; $1 \mu \mathrm{g} / \mathrm{ml}$ in A. dest.) directly on the mycelium and incubation for 15 min. FM4-64 fluorescence was recorded with a "chroma filter set 49005" (exciter ET545/30, emitter ET620/60, beam splitter T570lp). When indicated, vacuoles were stained with CMAC (7-amino-4-chloromethylcoumarin, CellTracker Blue; Invitrogen C-2110; stock solution $10 \mathrm{mM}$ in DMSO diluted to $\sim 10 \mu \mathrm{m}$ in A. dest.) and recorded with "chroma filter set 49000" (exciter D350/50, emitter ET560/50 and beam splitter T4001p). To stain nuclei 100$200 \mu \mathrm{l}$ of a DAPI (AppliChem, A1001) solution was applied to the mycelium and recorded with “chroma filter set 49000” (exciter D350/50, emitter ET560/50 and beam splitter T400lp). For this, DAPI was dissolved in A. dest. to a final concentration of $1 \mathrm{mg} / \mathrm{ml}$. This stock solution was then diluted with methanol to a concentration of $1 \mu \mathrm{g} / \mathrm{ml}$.

\subsubsection{Protein investigations}

\subsubsection{Protein preparation from S. macrospora}

To extract proteins from fungal mycelium the strains were inoculated in liquid BMM medium and grown for 3 days. The cell material was filtered with tissues, dried and grounded in a mortar with liquid nitrogen. The cells were lysed in the "Tissue Lyser" (Qiagen) by 30 Hrz for 3 min, with the help from $\sim 200 \mu$ glass beads $(\varnothing 0.25-0.5 \mathrm{~mm})$ and cold lysis buffer $(560 \mu \mathrm{l} / \mathrm{g}$ mycelium powder). The samples were separated from the cell debris at $13000 \mathrm{rpm}$ and $4{ }^{\circ} \mathrm{C}$ in 
a pre-cooled centrifuge for $15 \mathrm{~min}$ and were directly mixed with 5x SDS-PAGE loading dye to get a $1 \mathrm{x}$ dilution and heated at $95^{\circ} \mathrm{C}$ for $15 \mathrm{~min}$ for denaturation of the proteins (Laemmli, 1970). For the phosphorylation assay cell lysate was treated with phosphatase inhibitor cocktail as described by the manufacturer or $47 \mu \mathrm{l}$ cell lysate were treated with $1 \mu \mathrm{l}$ phosphatase puffer, $1 \mu \mathrm{MnCl} \mathrm{M}_{2}$ and $1 \mu \mathrm{l}$ lambda Phosphatase and incubated at $30^{\circ} \mathrm{C}$ for $1 \mathrm{~h}$.

\subsubsection{Co-immunoprecipitation (Co-IP)}

For Co-IP experiments the plasmids pSmnbr1-Dsred and pRS-egfp-Smatg8 were cotransformed into the $S$. macrospora wild type strain. Additionally, the complementation strain $\Delta$ Smnbr1::Smnbr1-Dsred ${ }^{\text {ect }}$ was constructed and used as control in this experiment as well as the already existing strain $\Delta$ Smatg8::egfp-Smatg8 ${ }^{\text {ect }}$ (Voigt and Pöggeler 2013a). For interaction studies of ribosomal proteins and SmNBR1 a wild type strain was used expressing SmNBR1-DsRED together with either SmRPL25-EGFP or SmRPS19-EGFP. As control, wild type strains expressing only SmRPL25-EGFP or SmRPS19-EGFP were used. The crude extracts were prepared as described in 2.2.8.1 and aliquots of $1 \mathrm{ml}$ were incubated at $4{ }^{\circ} \mathrm{C}$ for $1 \mathrm{~h}$ on a rotation device with $3 \mu \mathrm{l}$ monoclonal rat anti-EGFP (Chromotek 3H9 029762) or monoclonal rabbit anti-RFP (GeneScript A00682) antibody. The antigen-antibody complexes were recovered by adding protein A sepharose CL-4B beads (GE Healthcare Life Science, 170780-01) and incubated at $4{ }^{\circ} \mathrm{C}$ for an additional hour on a rotation device. Afterwards, the beads-lysate-mixtures were centrifuged for $2 \mathrm{~min}$ at $2000 \mathrm{rpm}$ and washed two times with dilution buffer. The samples were mixed with 5x SDS-PAGE loading dye to get a 1x dilution and boiled for $15 \mathrm{~min}$ at $95^{\circ} \mathrm{C}$. The preparation of SDS-PAGE, Western blotting and detection of the fusion proteins was done as described in 2.2.8.4 and 2.2.8.5.

\subsubsection{Protein preparation from S. cerevisiae}

To verify expression of proteins (AD- and BD-fusion proteins) of $S$. cerevisiae, yeast cell cultures were grown at $30^{\circ} \mathrm{C}$ to midlog phase $\left(\mathrm{OD}_{600} 0.8\right)$. Harvested cells were washed in 500 $\mu 1$ ice-cold buffer $b$ and lysed with glass beads $(\varnothing 0.25-0.5 \mathrm{~mm})$ in $500 \mu 1$ of buffer $b+$, and protein extracts were obtained via centrifugation at $13000 \mathrm{rpm}$ for $15 \mathrm{~min}$. Proteins were denatured in $5 \mathrm{x}$ SDS-PAGE loading dye at $95^{\circ} \mathrm{C}$ for $15 \mathrm{~min}$. The whole protein extracts were separated by SDS-PAGE using a $12 \%$ gel.

\subsubsection{Denaturing SDS-polyacrylamide gel electrophoresis (SDS-PAGE)}

12 or $15 \%$-SDS gels (12\% is representive shown in Table 6) were casted in a "MiniPROTEAN $^{\circledR}$ Tetra Cell” (Bio-Rad, Germany) and the chamber was filled with 1x SDS-PAGE 
running buffer. Once the samples were loaded the electric field of $80 \mathrm{~V}$ was applied and after the proteins passed the stacking gel, the voltage was increased to $140 \mathrm{~V}$. For every run a protein standard (Pageruler ${ }^{\mathrm{TM}}$ Prestained Protein Ladder) was used. After protein separation, the gel was further used for a Western blot on a nitrocellulose membrane.

Table 6: Composition of SDS-polyacrylamide gels.

\begin{tabular}{ccc}
\hline Components for one gel & $\begin{array}{c}12 \% \text { acrylamide } \\
\text { separation gel [ml] }\end{array}$ & $\begin{array}{c}5 \% \text { acrylamide } \\
\text { stacking gel [ml] }\end{array}$ \\
\hline Rotiphorese Gel 40 & 2.25 & 0.188 \\
1.5 M Tris/HCl pH 8.8 & 1.87 & - \\
0.5 M Tris/HCl pH 6.8 & - & 0.5 \\
$20 \%$ (w/v) SDS & 0.037 & 0.02 \\
A. dest. & 3.3 & 1.3 \\
TEMED & 0.006 & 0.0017 \\
$10 \%(w / v)$ APS & 0.05 & 0.013 \\
\hline
\end{tabular}

\subsubsection{Western blot, hybridization and detection reaction}

For Western blots, separated proteins were transferred from the SDS-PAGE gels onto nitrocellulose membranes using $1 \mathrm{x}$ transfer buffer and a Mini Trans-Blot ${ }^{\circledR}$ Cell device as described by the manufacturer (Biorad) (Towbin et al. 1979). After the transfer, the membrane was incubated for $1 \mathrm{~h}$ in $30 \mathrm{ml}$ skim milk blocking solution. The membrane was with the primary antibody solved in blocking solution incubated for at least $2 \mathrm{~h}$ at RT or overnight at $4^{\circ} \mathrm{C}$. Afterwards, the antibody was removed and the membrane was washed three times for 15 min with 1x TBST before the membrane was incubated with the secondary antibody diluted in blocking solution. After $1 \mathrm{~h}$ the secondary antibody was removed and the membrane underwent a second washing step.

Immune detection of the proteins containing an EGFP or DsRED tag was achieved using the following antibodies: monoclonal rat anti-EGFP antibody (1:4000, Chromotek 3H9 029762) and a secondary HRP-linked goat anti-rat antibody (1:5500, Invitrogen 62-9520); monoclonal rabbit anti-RFP (1:2000, GeneScipt A00682) and the appropriate secondary goat anti-rabbit antibody (1:2000, Life Technologies G21234). The immune detection of actin was performed with a monoclonal mouse anti-actin antibody (Novus, NB100-74340, 1:3000) and the secondary HRP-linked goat anti-mouse antibody (Dianova, 115-035-003, 1:10000). 
Immunodetection of aminopeptidase 1 (Ape1) was performed with a polyclonal rabbit antiApe1 antibody (kindly provided by M. Thumm, Göttingen, 1:2500) and as secondary antibody HRP-linked anti-rabbit antibody (1:2500, Invitrogen G21234) was used. Ubiquitin was detected with monoclonal mouse anti-ubiquitin (Millipore, ST1200, 1:1000) antibody and the secondary HRP-linked goat anti-mouse antibody (Dianova, 115-035-003, 1:10000).

SmATG12 and SmATG8 proteins fused to the Gal4-activation domain in the pGADT7 vector were detected using a monoclonal mouse anti-HA antibody (diluted 1:3000, Sigma-Aldrich A2095) and proteins fused to the Gal4-binding domain in the pGBKT7 vector were detected using a monoclonal mouse anti-myc antibody (1:5000, Cell Signaling Technology 2279). Blotting of proteins to the membrane was visualized by staining with ponceau red solution ( 3 $\%$ TCA, $0.2 \%$ ponceau S). The detection of the signals was performed with the Immobilon $^{\mathrm{TM}}$ Western HRP Substrate Kit and visualized on chemiluminescence films (Amersham Hyperfilm ${ }^{\mathrm{TM}}$ ECL) using an "Optimax X-ray film processor" (PROTEC GmbH \& Co. KG, Germany).

\subsubsection{GFP-Trap experiment and Coomassie staining}

The SmNBR1-EGFP fusion protein was used as bait for the GFP-Trap analysis. Protein extracts of the strains $\Delta$ Smnbr1::Smnbr1-egfp ${ }^{\text {ect }}$, containing the plasmid pSmnbr1-egfp expressing SmNBR1 C-terminally tagged with EGFP, and as control the wild type strain containing plasmid p1783-1 encoding the EGFP-tag under the control of the A. nidulans gpd promotor and $\operatorname{trp} C$ terminator, were prepared as described in 2.2.8.3 with an additional centrifugation step of $30 \mathrm{~min}$ for $13000 \mathrm{rpm}$ to get the protein lysate. $20 \mu \mathrm{l}$ GFP-Trap agarose beads were calibrated by washing them three times in cold $500 \mu \mathrm{l}$ dilution buffer at $3000 \mathrm{rpm}$ and $4^{\circ} \mathrm{C}$ for $2 \mathrm{~min} .1$ $\mathrm{ml}$ of the protein extracts was added to the beads and incubated at $4^{\circ} \mathrm{C}$ for $3 \mathrm{~h}$ in a spinning wheel to evoke end-over-end mixing. After centrifugation at $2000 \mathrm{rpm}$ and $4{ }^{\circ} \mathrm{C}$ for $2 \mathrm{~min}$, the supernatant was discarded and the pellet was washed and centrifuged two times with cold 500 $\mu l$ dilution buffer.

Trap beads were resuspended in $40 \mu 1$ 5x SDS-PAGE loading dye to get a $1 \mathrm{x}$ dilution and boiled at $95^{\circ} \mathrm{C}$ for $15 \mathrm{~min}$. After separation of the proteins by SDS-PAGE (2.2.8.4) the gel was stained with colloidal Coomassie follows (Kang et al. 2002). For Coomassie staining the gel was shaken in fixing solution for $1 \mathrm{~h}$ and subsequently washed twice with $\mathrm{H}_{2} \mathrm{O}$ for $10 \mathrm{~min}$. Afterwards, the gel was incubated overnight in Coomassie staining solution. To remove Coomassie particles after staining the gel was washed with $\mathrm{H}_{2} \mathrm{O}$. For destaining, the SDS-Gel was incubated in fixing solution and subsequently washed twice with $\mathrm{H}_{2} \mathrm{O}$. 


\subsubsection{Trypsin digest of proteins}

Protein digestion with trypsin was performed according to Shevchenko et al. (1996). Whole lanes of Coomassie stained gels were cut into 10 small pieces of approximately $2 \mathrm{~mm}$ and transferred into $1.5 \mathrm{ml}$ Protein LoBind Tubes (0030108116, Eppendorf, Hamburg, Germany). $30 \mu 1$ acetonitrile were added to the gel pieces. The volume was adjusted to the amount of gel, since the gel pieces should be fully covered with acetonitrile. The samples were incubated for 10 min under constant shaking before the acetonitrile was removed. The gel pieces were dried for $10 \mathrm{~min}$ in the SpeedVac concentrator (Eppendorf concentrator 5301). $150 \mu 110 \mathrm{mM}$ DTT were added and the samples were incubated at $56^{\circ} \mathrm{C}$ for $1 \mathrm{~h}$. The DTT solution was removed and the gel pieces were covered with $150 \mu \mathrm{l} 55 \mathrm{mM}$ iodoacetamide dissolved in $100 \mathrm{mM}$ $\mathrm{NH}_{4} \mathrm{HCO}_{3}$ followed by incubation for $45 \mathrm{~min}$ in the dark. The iodoacetamide was removed and the gel pieces were washed with $150 \mu 1100 \mathrm{mM} \mathrm{NH}_{4} \mathrm{HCO}_{3}$ for $10 \mathrm{~min}$ after removing $150 \mu \mathrm{l}$ acetonitrile were added and incubated for $10 \mathrm{~min}$. Both washing steps were performed under constant shaking and repeated twice. The gel pieces were dried in the SpeedVac concentrator for $10 \mathrm{~min}$ and subsequently covered with sufficient trypsin digestion buffer. The samples were incubated for $45 \mathrm{~min}$ on ice and excessive trypsin digestion buffer was removed. The gel pieces were covered with $25 \mathrm{mM} \mathrm{NH}_{4} \mathrm{HCO}_{3}$, and the samples were incubated overnight at $37^{\circ} \mathrm{C}$. The liquid was collected by centrifugation at $13000 \mathrm{rpm}$ and transferred to a fresh $1.5 \mathrm{ml}$ Protein LoBind Tube. Peptides were extracted from the gel by the sequential incubation with $20 \mathrm{mM}$ $\mathrm{NH}_{4} \mathrm{HCO}_{3}$ for $10 \mathrm{~min}$ and three times with a $50 \%$ acetonitrile $5 \%$ formic acid mixture for each $20 \mathrm{~min}$. Incubations were performed under constant shaking and the liquid was collected by centrifugation and transferred into the new LoBind Tube after each step. The collected supernatant was completely dried in the SpeedVac concentrator. The peptide pellet was resolved in $20 \mu \mathrm{l}$ sample buffer (2-5\% acetonitrile, $0.1 \%$ formic acid) for further purification. The purification with the $\mathrm{C} 18$ stop and go extraction (stage) tips was performed according to Rappsilber et al. (2003). Small disks were obtained from a C18 Solid phase extraction Disk and at least two of them were pushed into a 100-200 $\mu$ l pipette tip on top of each other. The C18 column material was equilibrated with $100 \mu 1$ methanol with $0.1 \%$ formic acid, followed by $100 \mu 170 \%$ acetonitrile with $0.1 \%$ formic acid and washed twice with $100 \mu 1 \mathrm{H}_{2} \mathrm{O}$ with $0.1 \%$ formic acid. After the addition of each solvent the stage tips were centrifuged with the help of an adaptor in $2 \mathrm{ml}$ reaction tubes at $13000 \mathrm{rpm}$ for $2 \mathrm{~min}$. The peptide samples were loaded and incubated on the stage tip for $5 \mathrm{~min}$, followed by centrifugation at $4000 \mathrm{rpm}$ for $5 \mathrm{~min}$. To increase the yield, the flow-through was loaded again and the stage tips were centrifuged at 
$4000 \mathrm{rpm}$ for 5 min once more. The $\mathrm{C} 18$ material was washed twice with $100 \mu 1 \mathrm{H}_{2} \mathrm{O}$ with $0.1 \%$ formic acid, each time followed by centrifugation at $10000 \mathrm{rpm}$ for $2 \mathrm{~min}$. For peptide elution $60 \mu 170 \%$ acetonitrile with $0.1 \%$ formic acid were applied onto the column, incubated on the stage tip for $5 \mathrm{~min}$, and centrifuged at $4000 \mathrm{rpm}$ for $5 \mathrm{~min}$. This peptide solution was dried completely in the SpeedVac concentrator and resolved in $20 \mu 1$ of the sample buffer for LC-MS analyses.

\subsubsection{Liquid chromatography-mass spectrometry analysis (LC/MS)}

LC/MS analysis for protein identification was performed with an "Orbitrap Velos Pro ${ }^{\mathrm{TM}}$ Hybrid Ion Trap-Orbitrap mass spectrometer". 1-5 $\mu$ l of peptide solutions were loaded and washed on an Acclaim ${ }^{\circledR}$ PepMAP 100 pre-column $(164564,100 \mu \mathrm{m}$ x $2 \mathrm{~cm}, \mathrm{C} 18,3 \mu \mathrm{m}, 100 \AA$, Thermo Fisher Scientific) with $100 \%$ loading solvent A $\left(98 \% \mathrm{H}_{2} \mathrm{O}, 2 \%\right.$ acetonitrile, $0.07 \%$ trifluoracetic acid (TFA)) at a flow rate of $25 \mu \mathrm{l} / \mathrm{min}$ for $6 \mathrm{~min}$. Peptides were separated by reverse phase chromatography on an Acclaim ${ }^{\circledR}$ PepMAP RSLC column $(75 \mu \mathrm{m}$ x $25 \mathrm{~cm}$, (164536) or $50 \mathrm{~cm}$ (164540), C18, $3 \mu \mathrm{m}, 100 \AA$, Thermo Fisher Scientific) with a gradient from $98 \%$ solvent $\mathrm{A}\left(\mathrm{H}_{2} \mathrm{O}, 0.1 \%\right.$ formic acid $)$ and $2 \%$ solvent $\mathrm{B}\left(80 \%\right.$ acetonitrile, $20 \% \mathrm{H}_{2} \mathrm{O}, 0.1$ $\%$ formic acid) to $42 \%$ solvent B for $95 \mathrm{~min}$ and to $65 \%$ solvent B for the following $26 \mathrm{~min}$ at a flow rate of $300 \mathrm{nl} / \mathrm{min}$. Peptides eluting from the chromatographic column were on-line ionized by nanoelectrospray at $2.4 \mathrm{kV}$ with the Nanospray Flex Ion Source (Thermo Fisher Scientific). Full scans of the ionized peptides were recorded within the Orbitrap FT analyzer of the mass spectrometer using a mass range of $300-1850 \mathrm{~m} / \mathrm{z}$ at a resolution of 30000 . Collision induced dissociation (CID) fragmentation of data-dependent top-ten peptides was performed with the "LTQ Velos Pro linear ion trap". For data acquisition and programming the XCalibur 2.2 software (Thermo Fisher Scientific) was used. Protein identification was performed with the MaxQuant 1.5.1.0 software (Cox et al. 2011) using a S. macrospora-specific peptide database (Nowrousian et al. 2010). The mass tolerance was $4.5 \mathrm{ppm}$ for precursor ions and 0.5 Da for fragment ions. The decoy mode was revert with a false discovery rate of 0.01 . Futher, data processing and statistical analysis were performed with the Perseus 1.5.1.0 software. A ttest was performed and only significant results were further considered. Database searches were performed with the SequestHT and Mascot search algorithms against a S. macrospora-specific protein database (Nowrousian et al. 2010). The digestion mode was set to trypsin and the maximum of missed cleavage sites to two. The decoy mode was revert with a false discovery rate of 0.01 . 


\subsubsection{Yeast-two hybrid (Y2H) interaction studies}

The $\mathrm{Y} 2 \mathrm{H}$ assay allows the investigation of the interaction of two proteins in yeast (Fields and Song 1989). Yeast cells were transformed with Y2H vectors that derived from pGADT7 and the pGBKT7 vectors (Clontech) which provided the LEU2 (yeast strain AH109) or TRP1 (yeast strain Y187) synthesis genes for the selection of transformed cells. Plasmids pGADT7 encoded the prey proteins, fused to the Gal4-activation domain, while pGBKT7 coded for the bait proteins, fused to the binding domain of the Gal4-transcription factor. The interaction of prey and bait protein activated the expression of reporter genes (ADE2, HIS3 and lacZ) controlled by the Gal4-transcription factor. For detection of the proteins in Western blot pGADT7 vector encodes for a HA-tag and pGBKT7 for a myc tag.

The prey plasmid pAD-Smnbr1 was constructed by ligation. For this the cDNA fragment of Smnbr1 was amplified using primer pair Nbr1-t1/Nbr1-t2, by this EcoRI-restriction sites were introduced at both ends. The resulting fragment was ligated into EcoRI-linearized vector pGADT7. To generate the bait vector pBD-Smnbr1, the same Smnbrl amplicon with EcoRI overhangs was cloned into the EcoRI-linearized vector pGBKT7. The different variants of Smnbrl were amplified with overhangs to this backbone vector as mentioned hereafter: fragment Smnbrl (1-843) with primers ADNbr1-(HR)-f/ADNbr1-LIR(HR)-r (2562 bp), Smnbrl (1-418) with primers ADNbr1-(HR)-f/ADNbr1-1-418(HR)-r (1315 bp) and Smnbrl (419-863) with primers ADNbr1-419-863(HR)-f/ADNbr1-(HR)-r. Further, the mutations Smnbrl ( $\left.\mathrm{T}^{843} \mathrm{~A}\right)$, Smnbrl ( $\left.\mathrm{Y}^{847} \mathrm{~A} \mathrm{~L}^{850} \mathrm{~A}\right)$, Smnbrl $\left(\mathrm{T}^{843} \mathrm{~A} \mathrm{Y}^{847} \mathrm{~A} \mathrm{~L}^{850} \mathrm{~A}\right)$ were introduced in the Smnbrl full-length fragment with primers Nbr1-T843A-f, Nbr1-Y847A-L850A-r (Smnbr1 $\left(\mathrm{T}^{843} \mathrm{~A}\right)$ served as template), and for Smnbrl $\left(\mathrm{S}^{793} \mathrm{~A}\right)$, Smnbrl $\left(\mathrm{S}^{793} \mathrm{~A} \mathrm{~S} \mathrm{~S}^{804} \mathrm{~A} \mathrm{~S}^{808} \mathrm{~A}\right)$ with primers Nbr1-SAcr-f2 and Nbr1-SAcr-f1, respectively, in a second PCR cycle. Afterwards, the fragments were cloned into EcoRI-hydrolyzed pGADT7 vector by homologous recombination in yeast (Colot et al. 2006) (2.2.7.1). Construction of plasmids pAD-Smatg8 and pBD-Smatg8 have been previously described in Voigt and Pöggeler (2013a).

The prey plasmid pAD-Smatg12 was constructed by cloning an amplified cDNA fragment of Smatg12 together with restriction sites NdeI and EcoRI using primer pair Atg12_Hf2/Atg12_Hr2, into NdeI/EcoRI-linearized vector pGADT7. To generate the bait vector pBD-Smatg12, the Smatg12 amplicon amplified with primer pair Atg12_Hf/Atg12_Hr with EcoRI and SalI overhangs was cloned into the EcoRI/SalI-linearized vector pGBKT7. Plasmids pAD-Smatg7 and pBD-Smatg7 were constructed by amplifying an Smatg7 cDNA fragment using primer pair Atg7_Hf/Atg7_Hr, which was ligated in EcoRI/HindIII-hydrolyzed 
pGADT7/pGBKT7 vectors, respectively. Plasmids pAD-Smatg3 and pBD-Smatg3 were obtained by amplifying Smatg3 with primer pair Atg3_Hf/Atg3_Hr from S. macrospora wt cDNA and inserting it as NdeI/EcoRI-fragment into appropriate hydrolyzed vectors pGADT7 and pGBKT7, respectively. All ligation reactions were conducted with T4-DNA ligase and the setup was as described in 2.2.4.2. Plasmids pAD-Smrpl25 and pBD-Smrpl25 were generated by homologous recombination in yeast. For this, Smrpl25 cDNA was amplified using primer pair AD-rpl25-f/AD-rpl25-r or BD-rpl25-f/BD-rp125-r to get a fragment of 612 bp including 29-bp overhangs, which was co-transformed with EcoRI-hydrolyzed pGADT7 and pGBKT7 vector into yeast, respectively. Plasmids pAD-Smrps19 and pBD-Smrps19 were constructed by amplifying a cDNA fragment of 470 bp using primer pair Smrps19-c5/Smrps 19-c3 for the pAD-Smrps19 and Smrps19-c5/Smrps19-c3-BamHI for the pBD-Smrps19 construct. The fragments were ligated in EcoRI/PstI-hydrolyzed pGADT7 or EcoRI/BamHI-hydrolyzed pGBKT7 vector.

S. cerevisiae strains AH109 and Y187 were used for yeast-two hybrid experiments. For yeasttwo hybrid analysis Mat $\alpha$ strain Y187 was transformed with bait plasmids pGBKT7, pBDSmatg8, pBD-Smnbr1, pBD-Smatg12, pBD-Smatg3, pBD-Smatg7, pBD-Smrpl25 or pBDSmrps 19 and transformants were selected for tryptophan prototrophy, whereas Mat a strain AH109 was transformed with prey plasmids pGADT7, pAD-ranBPM, pAD-Smatg8, pADSmatg12, pAD-Smatg3, pAD-Smatg7, pAD-Smrp125, pBD-Smrps19, pAD-Smnbr1, pADSmnbr1(1-418), pAD-Smnbr1(419-863), pAD-Smnbr1(1-843), pAD-Smnbr1( $\left.\mathrm{Y}^{847} A \mathrm{~L}^{850} \mathrm{~A}\right)$, pAD-Smnbr1( $\left.T^{843} A\right), \quad$ pAD-Smnbr1( $\left.T^{843} A \quad Y^{847} A \quad L^{850} A\right), \quad$ pAD-Smnbr1( $\left.{ }^{793} A\right), \quad$ pADSmnbr1( $\left.\mathrm{S}^{793} \mathrm{~A} \quad \mathrm{~S}^{804} \mathrm{~A} \quad \mathrm{~S}^{808} \mathrm{~A}\right)$ and transformants were selected for leucine prototrophy. Recombinant AH109 and Y187 strains were mated and selected on solid SD minimal medium lacking both, tryptophan and leucine. Alternatively, both plasmids were co-transformed into yeast strain AH109. Interaction of the bait and prey fusion constructs was confirmed by drop dilution assays and growth on selective SD minimal medium lacking histidine and adenine. Therefore, cells grown to log phase in liquid SD minimal medium without leucine and tryptophan were diluted to an optical density (OD) of 0.1 . From this main dilution, $20 \mu 1$ were spotted in a 1:10 dilution series onto selective SD plates, which were incubated at $30^{\circ} \mathrm{C}$ for five days. Yeast growth was visualized using a "Digital Microscope VHX-500F" (Keyence, Germany).

The PXG (pellet X-Gal) assay was performed and modified according to Möckli and Auerbach (2004). Yeast cultures were grown to $\mathrm{OD}_{600} 0.8$ and the cell pellet was solved in $100 \mu \mathrm{l}$ water and lyzed with glass beads ( $\varnothing 0.25-0.5 \mathrm{~mm}$ ) in a "Tissue Lyser" (Qiagen) by $30 \mathrm{Hrz}$ for 3 min. 
To $20 \mu \mathrm{l}$ of the cell pellet $100 \mu \mathrm{l}$ PBS buffer with $500 \mu \mathrm{g} / \mathrm{ml} \mathrm{X-Gal} \mathrm{and} 0.05 \%(\mathrm{v} / \mathrm{v}) \beta$ mercaptoethanol were added and incubated at RT for up to $12 \mathrm{~h}$. The $\beta$-galactosidase enzymatic activity was monitored by recording the color development with a 96-well microplate reader (Tecan Infinite 200).

\subsubsection{Yeast complementation and Ape1 maturation assay}

To analyze functional conservation of atg12 in S. macrospora and S. cerevisiae the aminopeptidase 1 (Ape1) maturation assay was carried out as described by Harding et al. (1995) and Voigt and Pöggeler (2013a). To express the Smatg12 cDNA under the control of the yeast MET25 promoter, a MET25-Smatg12 fusion construct was generated in pRS-met25-Smatg12. cDNA of S. macrospora wild type was used as template to amplify Smatg12 with primer pair Atg12_Cf/Atg12_Cr having SpeI- and SalI-restriction sites, respectively. The resulting 480-bp Smatg12 fragment was cloned into the SpeI/SalI-hydrolyzed plasmid pRS426-met25 (Mumberg et al. 1994). Plasmid pRS-met25-Scatg12 was generated by cloning a 561-bp Scatg 12 fragment amplified with primer pair Scatg12-f/Scatg12-r having SpeI- and EcoRI-restriction sites from S. cerevisiae BY4741 wt gDNA into the EcoRI/SpeI-hydrolyzed plasmid pRS426-met25.

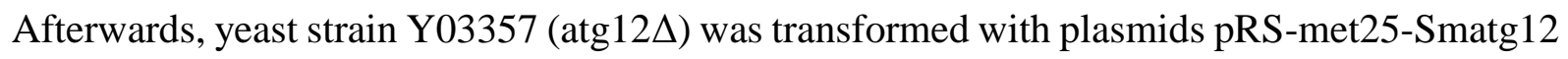
and pRS-met25-Scatg12. As negative controls, the S. cerevisiae wt strain BY4741 as well as the yeast atg12 $\Delta$ deletion strain, were transformed with the empty vector pRS426-met25. To test yeast transformants for their ability to rescue the Ape1 processing in the atg $12 \Delta$ mutant, cells were grown overnight in SD minimal medium and adjusted to $\mathrm{OD}_{600}=1$. One set of cells was used for protein extraction, while another set was grown for four hours in SD minimal medium lacking nitrogen (SD-N) to induce amino-acid starvation and, in turn, autophagy in S. cerevisiae (Harding et al. 1995). For protein extraction yeast cells were centrifuged (4000 $\mathrm{rpm}, 10 \mathrm{~min}, 4^{\circ} \mathrm{C}$ ) and washed with cold water and resuspended in $1 \mathrm{ml} \mathrm{A.} \mathrm{dest..} \mathrm{Subsequently,}$ cell suspension was mixed with $150 \mu \mathrm{l}$ lysis buffer (S. cerevisiae) and incubated for $10 \mathrm{~min}$ on ice. $50 \%$ TCA was added and the suspension was incubated for additional $10 \mathrm{~min}$ on ice. After centrifugation $\left(4000 \mathrm{rpm}, 10 \mathrm{~min}, 4^{\circ} \mathrm{C}\right)$, the cell pellet was washed twice with acetone $\left(-20^{\circ} \mathrm{C}\right)$ and resuspended in $100 \mu 15 x$ SDS-PAGE loading dye (Laemmli 1970) and incubated at $95^{\circ} \mathrm{C}$ for $10 \mathrm{~min}$. Samples were centrifuged at $4000 \mathrm{rpm}$ for $10 \mathrm{~min}$ at $4^{\circ} \mathrm{C}$ before separating $10-15$ $\mu 1$ of the protein solution by $12 \%$ SDS-PAGE, followed by blotting onto a nitrocellulose membrane as described in 2.2.8.4. Immunodetection of aminopeptidase 1 (Ape1) was performed with a polyclonal rabbit anti-Ape1 antibody (2.2.8.5). 


\subsection{Measures of safety}

Genetic engineering experiments of security level 1 have been conducted according to the guide lines of the genetic engineering law (GenTG) stated on 16.12.1993 (recently altered by Art. 12 G v. 29.7.2009 I 2542). 


\section{Results}

\subsection{Characterization of the autophagy-associated protein SmATG12 in}

\section{S. macrospora}

\subsubsection{Identification of a S. macrospora ATG12 homolog as an interaction partner of SmATG7 and SmATG3}

The ubiquitin-like gene Smatg 12 is one of the conserved core autophagy genes. Its function was not yet analyzed in S. macrospora. A BLASTP search of predicted S. macrospora proteins (Nowrousian et al. 2010) using S. cerevisiae ATG12 (P38316) as a query sequence identified a protein of 215 amino acids encoded by the ORF SMAC_06998 as the top hit $\left(3 \mathrm{e}^{-13}\right)$. Compared with ATG12 proteins from other organisms, the protein SMAC_06998 has an extended $\mathrm{C}$-terminal region but no glycine residue at the $\mathrm{C}$-terminus. Automatic annotation predicted two introns in SMAC_06998 (data not shown); however, RT-PCR amplification with primer pair 06998_f/06998_r and sequencing confirmed only splicing of the first intron, which leads to an earlier stop codon. The amplified cDNA encodes a protein of 159 amino acids with a conserved C-terminal glycine that shares a significant level of sequence similarity with ATG12 proteins of plants and animals that also have a conserved glycine at the C-terminus (Figure 8). The S. macrospora ATG12 homolog shares $81 \%$ and $50 \%$ identity to the previously described ATG12 proteins from N. crassa and Magnaporthe oryzae (Kershaw and Talbot 2009; Chinnici et al. 2014), but only about $20 \%$ identity with homologs from $S$. cerevisiae, plants and animals. However, most of the residues involved in non-covalent interactions between ATG12 and ATG5 (Supplement 1), binding and conjugation of ATG3 (Supplement 2), or binding of ATG8 in the S. cerevisiae and the human ATG12 homologs are conserved in the S. macrospora ATG12 (Radoshevich et al. 2010; Metlagel et al. 2013; Noda et al. 2013; Otomo et al. 2013; Kaufmann et al. 2014). Several residues within the BH3 domain of ATG12 homologs from mammals and non-mammalian vertebrates are also conserved. Similar to the S. cerevisiae and other fungal ATG12 homologs, a conserved aspartic acid residue (equivalent to D64 in human ATG12) that is required for binding of anti-apoptotic proteins, is replaced by a serine residue at position 82 in the S. macrospora protein (Figure 8) (Rubinstein et al. 2011). 

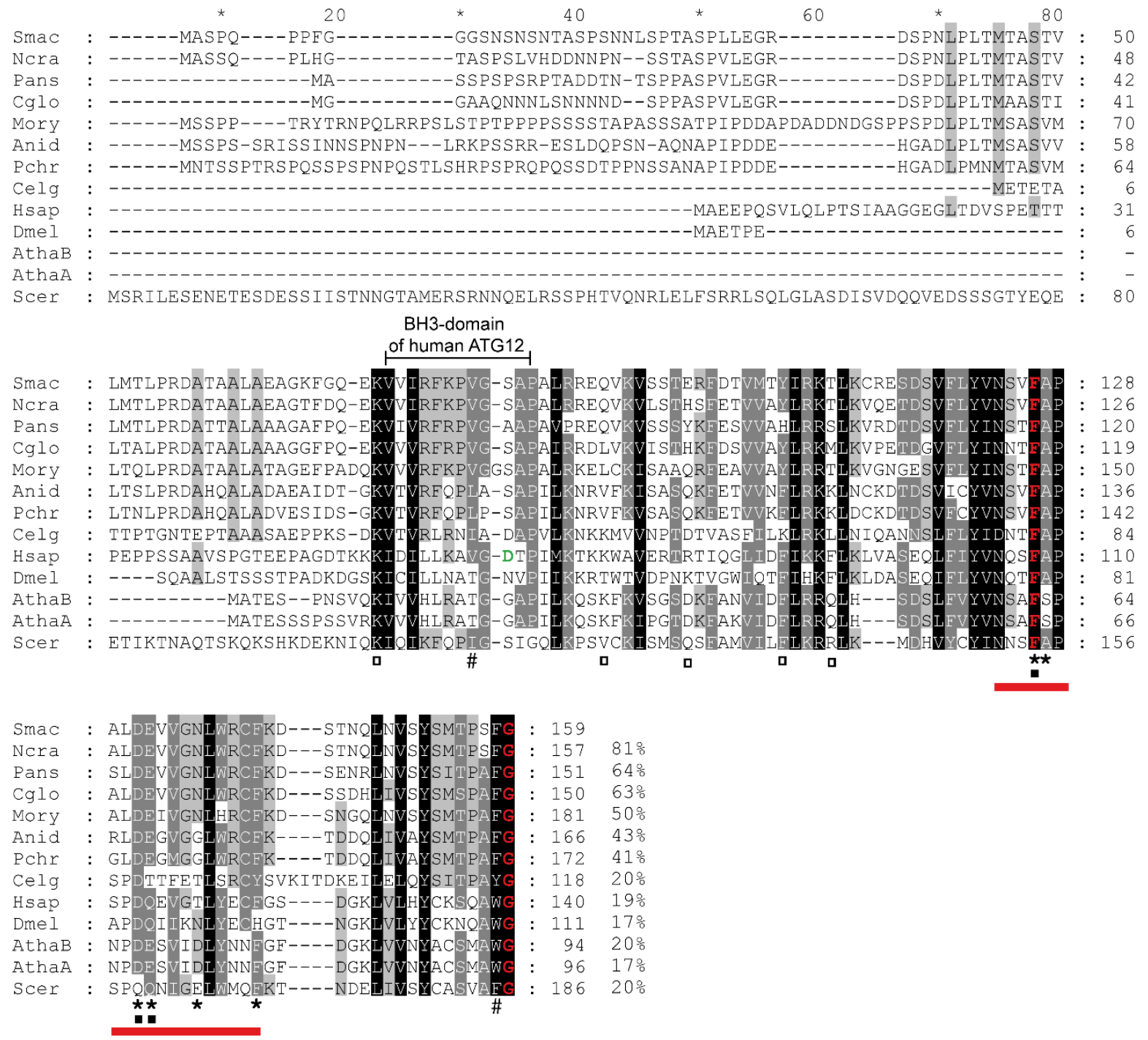

Figure 8: Multiple-sequence alignment of ATG12 orthologs from fungi, plants and animals.

ClustalX alignment was created using the following sequences: Smac, Sordaria macrospora (XP_003349162.1), excluding 56 C-terminal amino acids; Ncra, Neurospora crassa (Q7S083.1); Pans, Podospora anserina, (XP_001906089.1); Cglo, Chaetomium globosum (Q2GSG9.2); Mory, Magnaporthe oryzae (XP_368646.1); Anid, Aspergillus nidulans (Q5BCH0.2); Pchr, Penicillium chrysogenum (XP_002557636.1); Celg, Caenorhabditis elegans (CCD61524.1); Hsap, Homo sapiens (NP_004698.3); Dmel, Drosophila melanogaster (NP_648551.39); AthaB, Arabidopsis thaliana (Q9LVK3.1); AthaA, A. thaliana (Q8S924.1); Scer, Saccharomyces cerevisiae (P38316). Identical amino acids, which are conserved in all proteins, are shaded in black; residues conserved in at least 10 of 13 sequences are shaded in dark grey and residues conserved in at least eight sequences are shaded in light grey. The conserved C-terminal glycine residue for the covalent linkage to ATG5 and the conserved phenylalanine residue corresponding to Phe154 in the S. cerevisiae Atg12 is labeled in red (Hanada and Ohsumi 2005), amino acids important for non-covalent interactions between ATG12 and ATG5 in S. cerevisiae according to Noda et al. (2013) are marked by asterisks. Non-covalent contacts between ATG12 and ATG5 identified in the human homologs according to Otomo et al. (2013) are marked by black squares. The red bar represents the turn-loop-alpha helix 2 segment (Asn105-Phe123 of the human ATG12) which is associated with the interaction surface of ATG5 (Otomo et al. 2013). White squares mark residues of the human ATG12, which are important for binding of ATG3 (Metlagel et al. 2013); \#, indicates residues of the non-canonical AIM of ATG12 involved in interaction with ATG8 (Kaufmann et al. 2014). The region of the BH3 domain identified in the human ATG12 homolog is indicated and the conserved aspartic acid residue is indicated in green. Aminoacid identity in $\%$ is given at the right margin. 
In S. cerevisiae, direct protein-protein interactions between the UBL protein Atg12 and both E1/E2-like enzymes Atg7 and Atg3 have been reported (Yamazaki-Sato et al. 2003; Metlagel et al. 2013; Otomo et al. 2013). To confirm that SmATG12 can interact with SmATG7 (SMAC_06539) and SmATG3, yeast-two hybrid analyses were performed after cloning of Smatg12, Smatg7 and Smatg3 (SMAC_05399) cDNAs into prey and bait vectors pGBKT7 and pGADT7. The resulting bait and prey plasmids were transformed into yeast strains Y187 and AH109, respectively, which were then mated, or both plasmids were co-transformed into strain AH109. Transactivation of pBD-derivatives was tested by mating with the AH109 strain carrying the empty vector pGADT7 (data not shown). As a positive control and to confirm expression of the proteins encoded by the bait plasmids, strains were mated with yeast strain AH109 containing pAD-ranBPM. RanBPM directly interacts with the Gal4-binding domain, which provides a method for confirming that the gene cloned into the bait vector is expressed appropriately (Tucker et al. 2009). The two-hybrid experiment clearly demonstrated an interaction between SmATG12 and both SmATG3 and SmATG7 (Figure 9A+B).

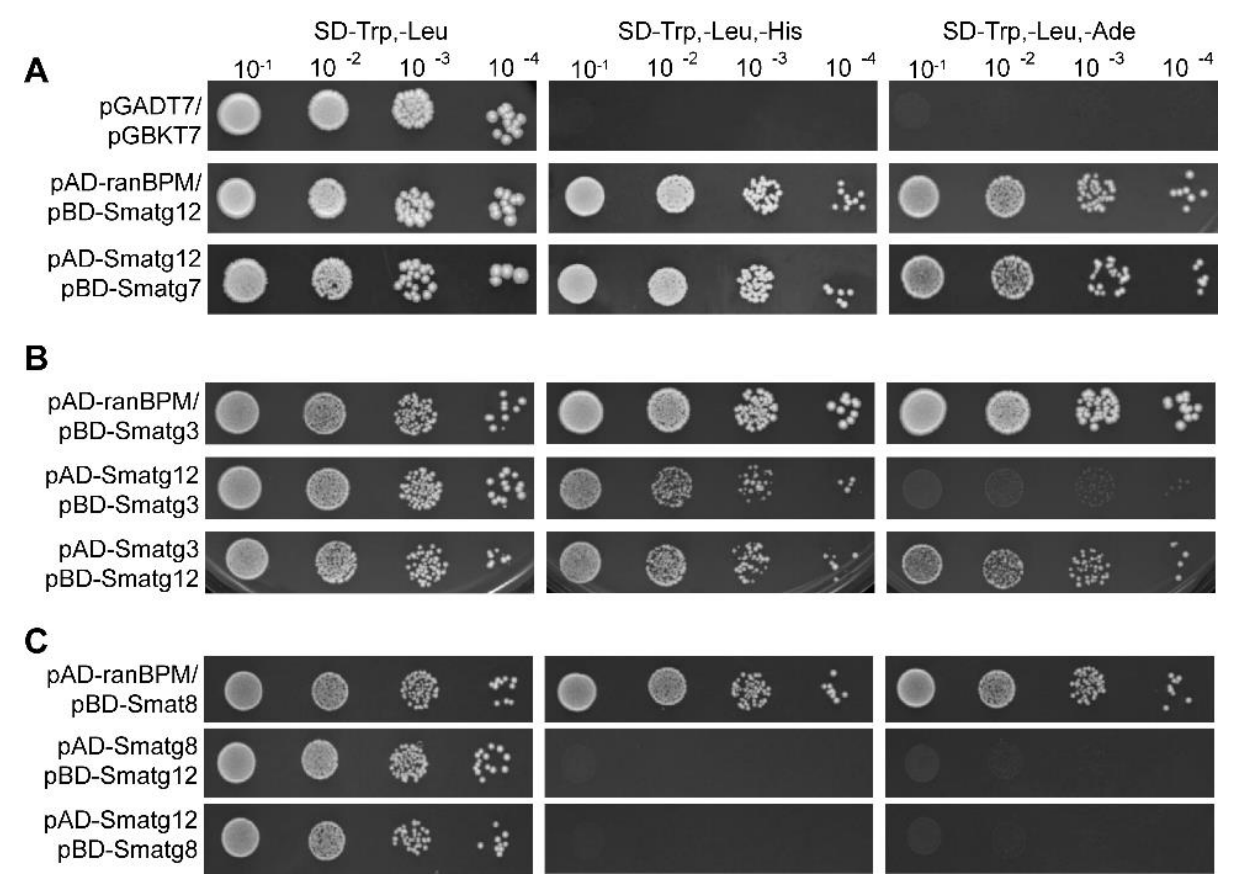

Figure 9: Yeast-two hybrid interaction of SmATG12 with SmATG7, SmATG3 and SmATG8.

Full-length cDNAs of Smatg12, Smatg7 and Smatg3 were used to generate Gal4-DNA binding domain (BD) and activation domain (AD) plasmids. Smatg8 two-hybrid vectors were previously described in Voigt and Pöggeler (2013a). To select for the presence of both plasmids $20 \mu \mathrm{l}$ of cells were spotted in serial dilutions on SD medium lacking tryptophan and leucine (SD -Trp, -Leu) or to verify the interactions of the proteins on medium lacking additionally histidine or adenine (SD -Trp, -Leu, -His/-Ade). (A) SmATG12 and SmATG7 interacted only when SmATG7 was expressed as Gal4-BD fusion protein. (B) SmATG12 and SmATG3 interacted with each other as bait and prey proteins, respectively. (C) SmATG8 did not interact with SmATG12. Transformants carrying a bait plasmid and pAD-ranBPM (Tucker et al. 2009) were used to confirm the appropriate expression of the bait proteins. Strains carrying empty plasmids pGADT7 and pGBKT7 served as negative control (A). Plates were incubated for $3-5$ days at $30^{\circ} \mathrm{C}$. 
Nevertheless, SmATG12 and SmATG7 interacted only when SmATG7 was expressed as a Gal4-BD fusion protein (Figure 9A). Recently, Kaufmann et al. (2014) demonstrated that the yeast Atg8-PE can directly interact with Atg12 via a non-canonical AIM in Atg12. However, interaction of SmATG12 and SmATG8 could not be demonstrated in the yeast-two hybrid system (Figure 9C), although expression of SmATG8 and SmATG12 in yeast was verified by Western-blot analysis (Figure 10).
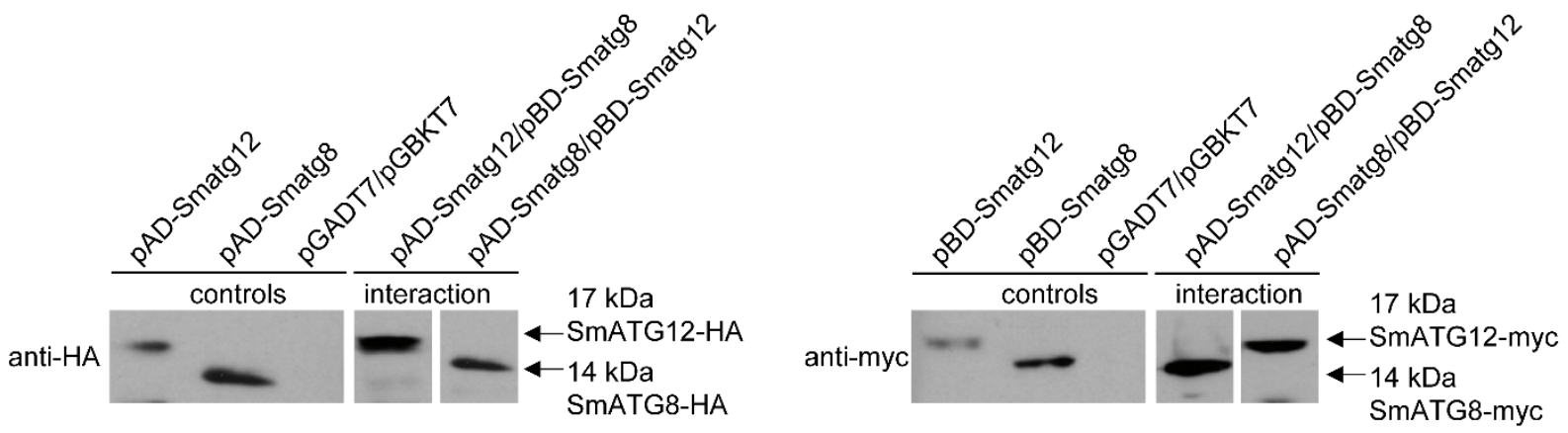

Figure 10: Verification of the protein expression of SmATG12 and SmATG8 in yeast-two hybrid transformants.

The proteins SmATG12 and SmATG8 fused to the Gal4-activation domain (AD) were detected with an anti-HA antibody and those fused to the Gal4-binding domain (BD) with an anti-myc antibody. Transformants carrying either a single vector or both vectors were used as positive and negative controls, respectively.

Furthermore, in this study we tested the functional conservation of SmATG12 and its yeast counterpart by a yeast complementation assay with the Smatg12 cDNA expressed under the control of a MET25 promoter in an S. cerevisiae atg12 $\Delta$ null mutant. Rescue of autophagy in the S. cerevisiae mutant was monitored using an aminopeptidase I (Ape1) maturation assay based on the autophagy-dependent maturation of the precursor proaminopetidase I (prApe1) to the mature enzyme (mApe1) (Harding et al. 1995). In S. cerevisiae, Ape1 maturation relies on an intact UBL protein Atg12. However, the Smatg12 gene was unable to complement the yeast $\operatorname{atg} 12 \Delta$ mutant (Figure 11).

In S. cerevisiae, Ape1 is delivered by the cytoplasm-to-vacuole targeting pathway to the vacuole. So far, it is not clear if this pathway exists in S. macrospora. In addition, we therefore monitored autophagy in the complemented mutant atg12 $\Delta$ by the GFP-ScAtg8 proteolysis assay. When GFP-ScAtg8 is delivered to the lumen of the vacuole, the Atg8 part of the fusion protein is sensitive to degradation, whereas the GFP moiety is relative resistant to hydrolysis. 


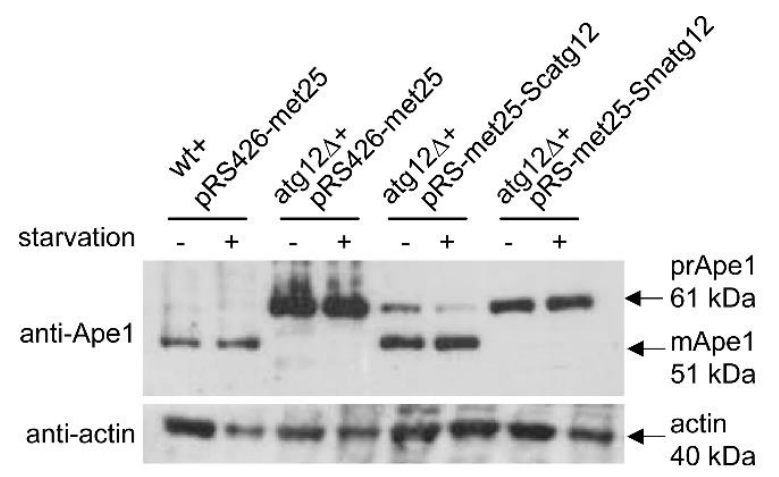

Figure 11: Complementation of the $S$. cerevisiae atg12 $\Delta$ autophagy mutant with the $S$. macrospora Smatg12 gene.

Complementation of the yeast strain atg12 $\Delta$ with Smatg12 under the control of the yeast MET25 promoter (pRSmet25-Smatg12) was analyzed using an aminopeptidase 1 (Ape1) maturation assay (Harding et al. 1995). As positive controls, the $S$. cerevisiae wt strain BY4741 was transformed with the empty plasmid pRS426-met25 or atg12 $\Delta$ was transformed with the yeast plasmid pRS-met25-Scatg12 expressing the endogenous Scatg12 gene. $S$. cerevisiae atg $12 \Delta$ transformed with the empty vector pRS426-met 25 served as negative control. Total cell extracts of an equal amount of yeast cells of a growing culture under non-starvation conditions (-) or 4h nitrogen starved cells (+) were separated on $15 \%$ SDS-PAGE. After blotting, nitrocellulose membranes were probed with an anti-Ape1 antibody. Anti-actin antibody was used as loading control. prApe1, Ape1 precursor; mApe1, mature Ape1.

In a Western blot with an anti-GFP antibody the appearance of free GFP can be used to monitor delivery of autophagosomal membranes to the vacuole (Klionsky et al. 2012). The assay revealed that also no processing of GFP-ScAtg8 occurred in the atg12 $\Delta$ yeast strain complemented with the Smatg12 gene (Figure 12).

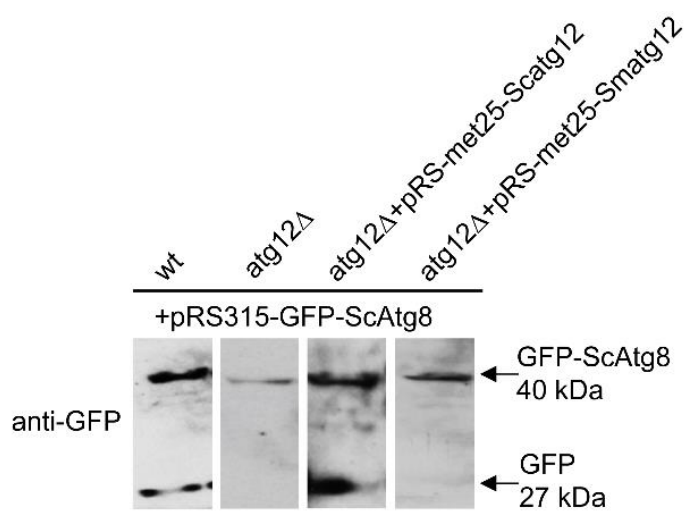

Figure 12: Proteolytic cleavage of GFP-ScATG8.

The wt (BY4741), atg12 $\Delta$ and the putative complementation strains atg12 $\Delta+$ pRS-met25-Scatg12 and $\operatorname{atg} 12 \Delta+$ pRS-met25-Smatg12 were transformed with plasmid pRS315-GFP-ScAtg8. The GFP-ScAtg8 protein was detected with an anti-GFP antibody and has an expected size of $40 \mathrm{kDa}$. The proteolytic cleavage of the GFPScAtg8 fusion protein can be detected by the free GFP signal at $27 \mathrm{kDa}$. 


\subsubsection{Deletion of Smatg12 impairs vegetative growth and fruiting-body development}

To analyze the effect of Smatg12 on autophagy, vegetative growth and sexual development of $S$. macrospora, a $\Delta$ Smatg 12 knockout mutant was generated for phenotypic characterization (Figure 13). The Smatg12 knockout strain was constructed as described in 2.2.6.2. The EGFPSmATG8 proteolysis assay in the $S$. macrospora $\Delta$ Smatg12 mutant revealed that vacuolar degradation of the fusion protein is inhibited, whereas in the complemented $\Delta$ Smatg8 strain $\left(\Delta\right.$ Smatg8::egfp-Smatg8 ${ }^{\text {ect }}$ ) proper vacuolar proteolysis of the fusion protein can be observed (Figure 14).

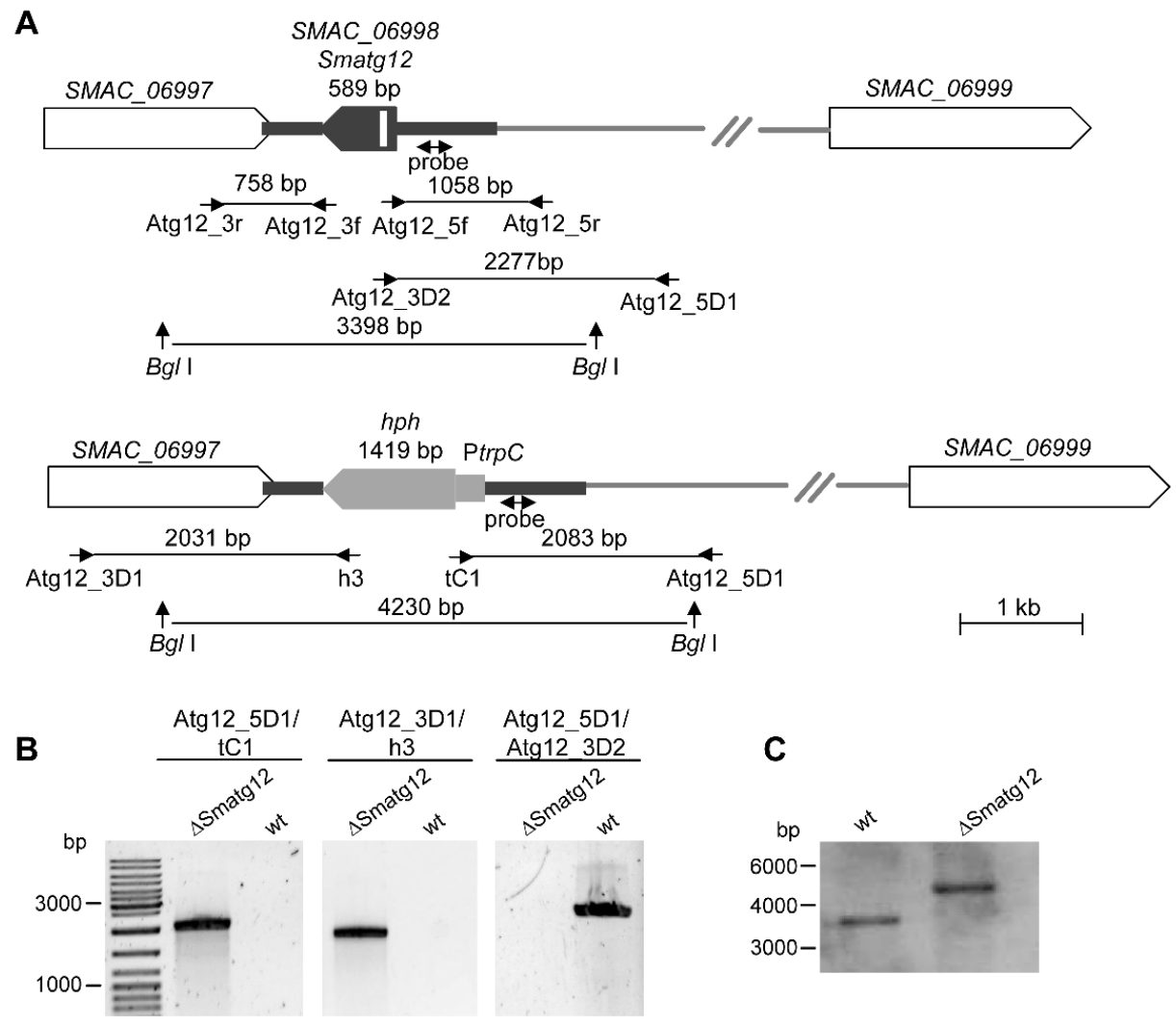

Figure 13: Construction of a $\Delta$ Smatg12 mutant.

(A) Schematic illustration of the S. macrospora Smatg12 locus SMAC_06998 (black arrow) with neighboring genes SMAC_06997 and SMAC_06999 and the derived $\triangle$ Smatg12 knockout locus. Gene replacement was reached by integration of an $h p h$-deletion cassette at the respective gene locus (grey arrow) by homologous recombination. The intron is indicated as white box. Positions of primers used for knockout plasmid construction and verification of the successful homologous integration at the Smatg12 locus are marked with small arrows. Sizes of the corresponding PCR fragments are indicated. $h p h$, hygromycin resistance; PtrpC, A. nidulans trpC promoter. (B) PCR analysis for verification of the integration of the $h p h$-deletion cassette at the desired Smatg 12 gene locus in comparison to the wt. The positions of primers and expected fragment sizes indicated in (A) could be detected. (C) Southern hybridization for verification of the $\Delta$ Smatg12 strain. Genomic DNA from S. macrospora wt and the $\Delta$ Smatg 12 mutant was digested with $B g I I$ and hybridized with the 300-bp probe indicated in (A), which covered the promoter region of Smatg 12. The expected fragments of $3398 \mathrm{bp}$ for the wt and $4230 \mathrm{bp}$ for the $\Delta$ Smatg12 strain could be detected. The scale bar indicates $1 \mathrm{~kb}$. 
Although, Smatg 12 cannot complement the $S$. cerevisiae atg $12 \Delta$ strain, this result suggests, that the isolated gene is an ortholog of the yeast Scatg12.

In contrast to the wild type strain and a complemented mutant strain ( $\Delta$ Smatg12::egfpSmatg12 ect), the mutant $\Delta$ Smatg12 did not form mature fruiting bodies when grown on solid SWG fructification medium or under histidine-starvation conditions imposed using the drug 3aminotriazole (3-AT) (Figure 15A). Prevention of autophagy is known to lead to the impairment of the foraging capability of filamentous ascomycetes (Josefsen et al. 2012), which describes the growth of filamentous fungi over a non-nutritious surface in order to reach nutrient-rich regions. The required nutrients are thought to be provided by autophagy taking place in the basal hyphae of mycelia (Shoji and Craven 2011; Voigt and Pöggeler 2013b; Chinnici et al. 2014).

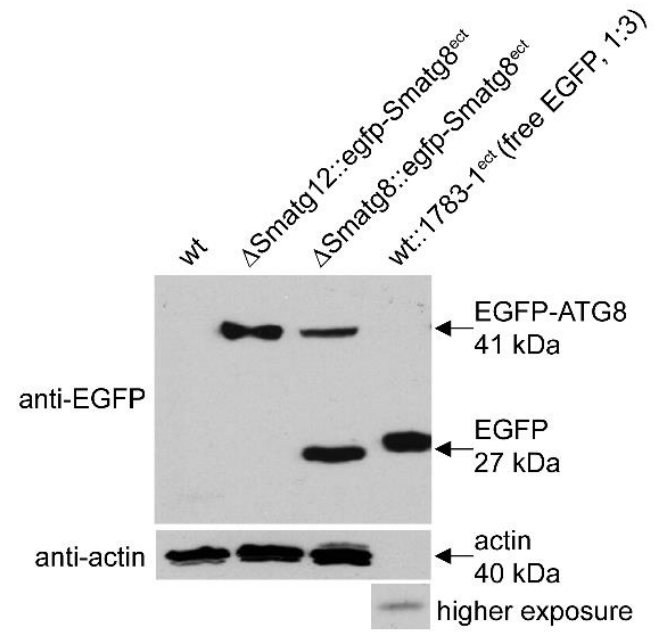

Figure 14: EGFP-SmATG8 protein degradation in the
$\Delta$ Smatg12 strain compared to the corresponding
complemented $\Delta$ Smatg8::egfp-Smatg8 ${ }^{\text {ect }}$ strain.
Protein crude extracts of $S$. macrospora wt, wt::1783- $1^{\text {ect }}$,
$\Delta$ Smatg12::egfp-Smatg8 ${ }^{\text {ect }}$ and $\Delta$ Smatg8::egfp-Smatg8 $8^{\text {ect }}$
strains expressing EGFP or EGFP-SmATG8 were separated
on a $12 \%$ SDS-PAGE gel. The Western blot hybridization
using an anti-EGFP antibody verified the degradation of the
EGFP-SmATG8 fusion protein in the complemented
$\Delta$ Smatg8 strain by accumulation of free EGFP, whereas in
the $\Delta$ Smatg12 mutant the EGFP-SmATG8 fusion protein
accumulated. Wt and wt::1783- ${ }^{\text {ect }}$ were used as controls.
Actin was detected as protein loading control.

To analyze the foraging capability of $\Delta \operatorname{Smatg} 12$, wild type and the complemented strain ( $\Delta$ Smatg12::egfp-Smatg12 ${ }^{\text {ect }}$ ), agar plugs were transferred into an empty cell-culture plate and incubated for 5 days at $27^{\circ} \mathrm{C}$. While the wild type and the complemented strain underwent extensive mycelial growth, $\Delta$ Smatg12 was unable to grow over the inert plastic surface (Figure 15B). In addition, the deletion mutant displayed a significant decrease in growth rate under histidine-starvation conditions imposed by 3-AT (Figure 15C). In the complemented strain the growth defect was only partially complemented in comparison to the wild type, probably due to an ectopic integration of the egfp-tagged Smatg12. Similarly, a slight growth defect of the complemented mutant could be observed under non-starvation conditions (SWG fructification medium), which might be also explained by ectopic integration of the egfp-tagged Smatg12 wild type copy. 
A

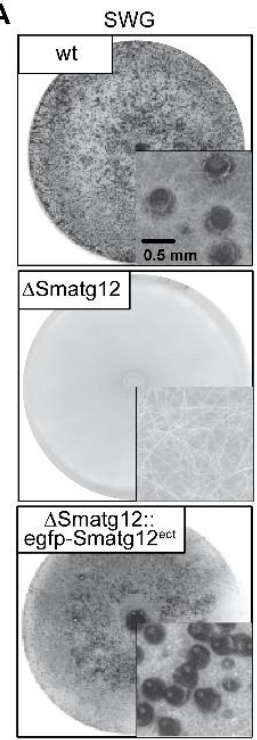

SWG +2.5 mM 3-AT

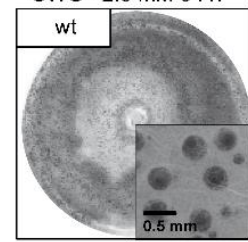

$\Delta$ Smatg12

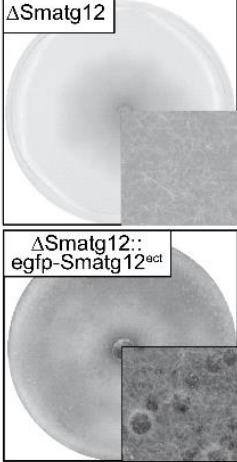

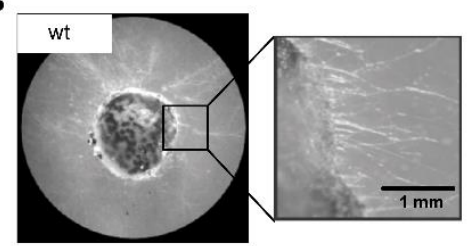
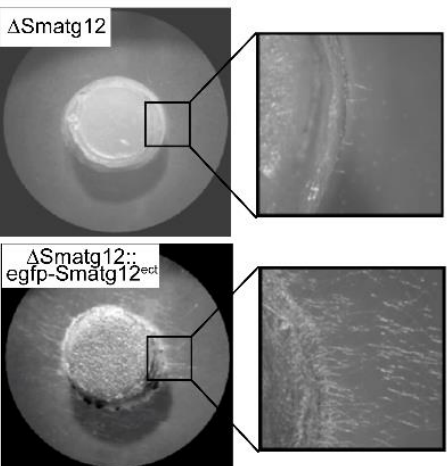

C

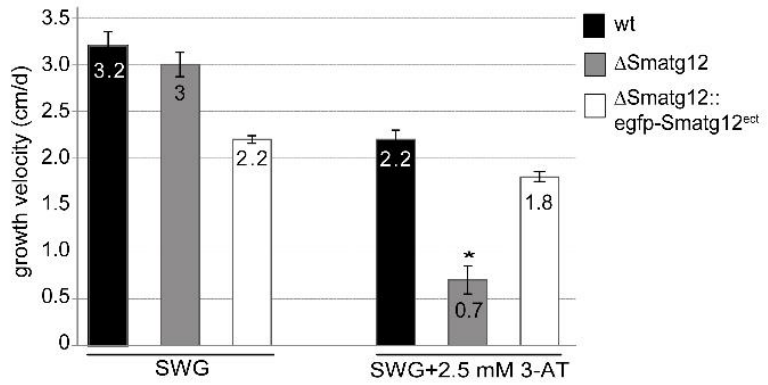

D $\begin{array}{lll}3 \mathrm{~d} \text { ascogonium } & 4 \mathrm{~d} & 5 \mathrm{~d} \text { pigmented } \\ \text { protoperithecium } & \text { protoperithecium }\end{array}$

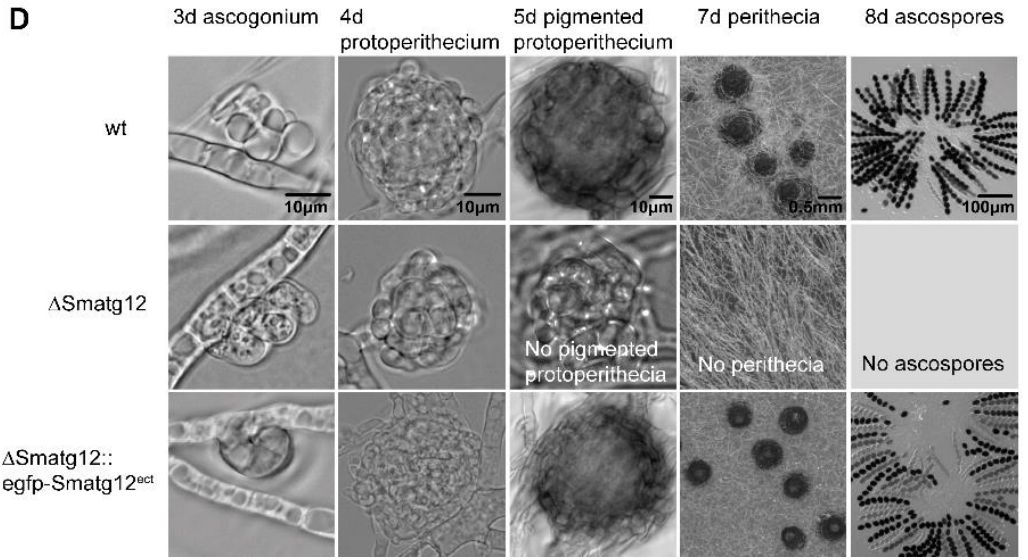

Figure 15: Phenotypic characterization of $S$. macrospora wt, Smatg12 deletion and complementation strain. (A) Phenotype of wt, $\Delta$ Smatg12 and rescued strain $\Delta$ Smatg12::egfp-Smatg $12^{\text {ect }}$ grown on SWG or SWG medium supplemented with $2.5 \mathrm{mM} 3$-AT in petri dishes. Insets show a detailed view of perithecia or sterile mycelium. The images were taken seven days post inoculation. (B) Foraging capacity of indicated strains. Agar plugs of 0.5$\mathrm{cm}$ diameter were transferred into empty cell-culture plates $(6$ well, $17.2 \mathrm{ml})$ and incubated for $5 \mathrm{~d}$ at $27^{\circ} \mathrm{C}$ in a damp chamber before photographed. (C) The growth velocity of wt, $\Delta$ Smatg 12 and the complemented strain was analyzed by measuring the growth velocity in $\mathrm{cm} / \mathrm{day}$ in $30-\mathrm{cm}$ race tubes. Growth rates on SWG medium shown are averages from 7 independent measurements of three independent experiments $(n=21)$, standard deviations are indicated by error bars. Asterisk indicates significant difference to wt according to Student's t-test $(\mathrm{p}<0.0000001)$. (D) Microscopic investigation of sexual development of $\Delta$ Smatg12 compared to wt and the complemented strain. Strains were grown on SWG medium. Expression of the EGFP-SmATG12 fusion construct complemented the sterile phenotype of $\Delta \operatorname{Smatg} 12$. The wt and the complemented strain $\Delta$ Smatg12::egfp- Smatg12 ${ }^{\text {ect }}$ form ascogonia at day 3 , and protoperithecia at day 4 post inoculation. These develop to pigmented protoperithecia at day 5 and to mature perithecia at day 7 . Sexual development of the mutant $\Delta$ Smatg12 is blocked at the stage of protoperithecia formation. $\Delta$ Smatg 12 neither forms pigmented protoperithecia nor perithecia and ascospores. Scale bars as depicted. 
Microscopic investigation of the fruiting-body development revealed that the mutant was arrested at the protoperithecia-developmental stage and was unable to form mature fruiting bodies or ascospores (Figure 15A+D). Altogether, five independent homokaryotic deletion mutants and two independent complemented mutants which displayed homogenous phenotypes were analyzed. Thus, the observed phenotypes are associated with the deletion of the Smatg12 gene.

\subsubsection{EGFP-SmATG12 localizes to phagophore assembly sites}

To investigate the in vivo localization of SmATG12, the complementation strain $\Delta$ Smatg12::egfp-Smatg12 ${ }^{\text {ect }}$ was used. The ectopically integrated egfp-Smatg12 fusion construct, under the control of the endogenous promoter, was able to complement the sterile phenotype of $\Delta$ Smatg12 (Figure 15). Fluorescence microscopy of the functionally expressed EGFP-SmATG12 fusion protein in the proliferating mycelium ( $24 \mathrm{~h}$ after inoculation) revealed localization in the cytoplasm as small discrete dots or cup-shaped structures that were presumed to be phagophore assembly sites or growing phagophores (Figure 16A). In the $\Delta$ Smatg8 mutant, the EGFP-SmATG12 fluorescence signals appeared larger and more distinct than that of the complemented $\Delta$ Smatg12 mutant (Figure 16B). To investigate the role of SmATG12 in autophagy, the autophagy-mediated engulfment of EGFP-labeled SmATG8 into the vacuole was examined. Transformation of pRS-egfp-Smatg8 (Voigt and Pöggeler 2013a) into $\Delta$ Smatg12 allowed us to follow the fluorescence signal of EGFP-labeled autophagosomes. In contrast to the complemented $\Delta$ Smatg8 mutant, in the $\Delta$ Smatg12 mutant EGFP-SmATG8 fluorescence was diffused throughout the cytoplasm, and large cytoplasmic aggregates were excluded from vacuoles, even in basal hyphae (Figure 16D). 
A

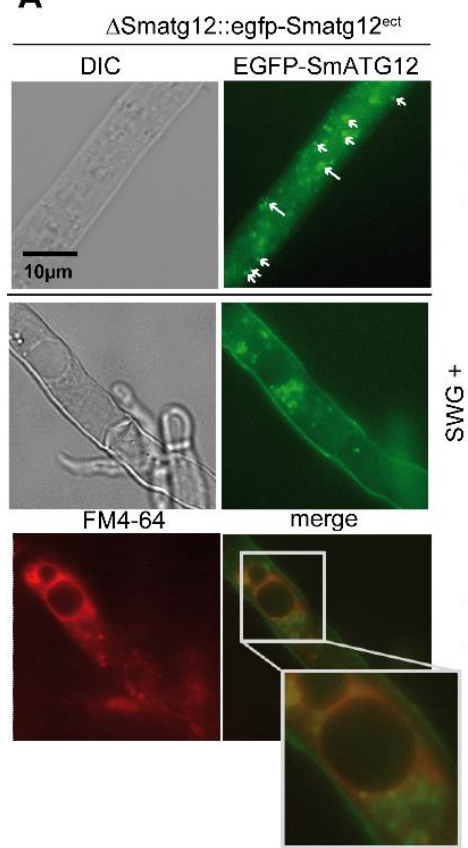

B

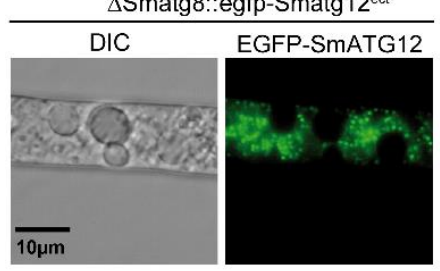

C

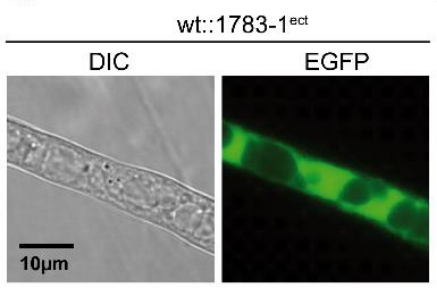

D

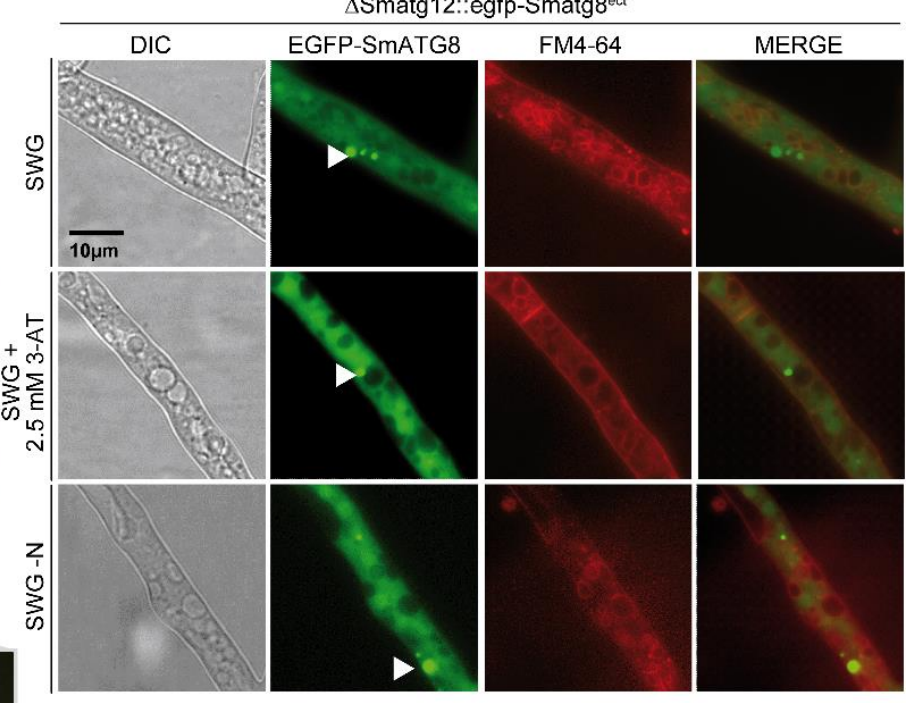

E

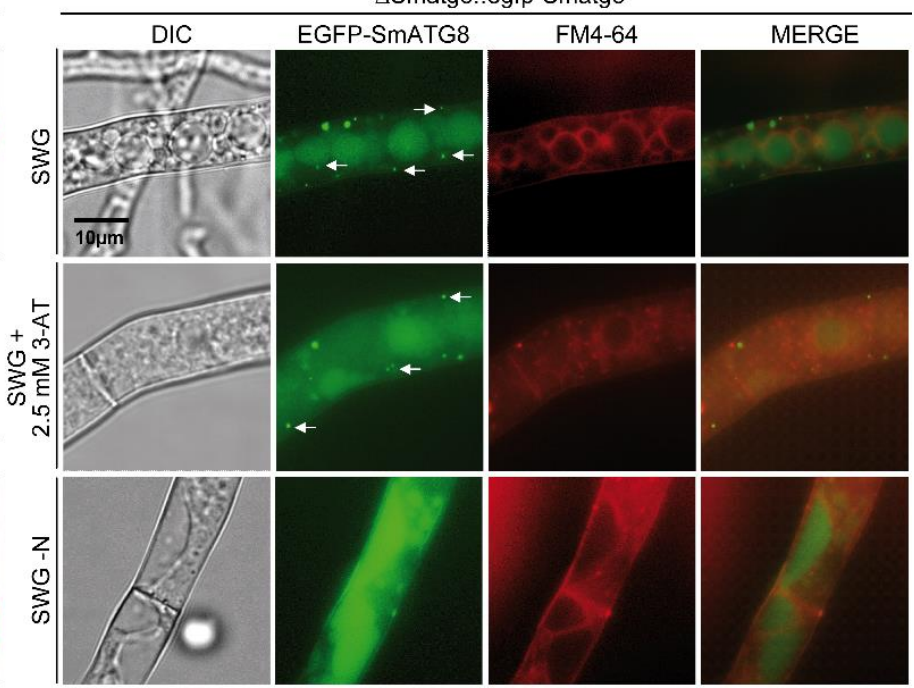

Figure 16: Fluorescence microscopic localization of EGFP-SmATG12 and EGFP-SmATG8.

(A) Mutant $\Delta$ Smatg12 was transformed with plasmid pegfp-Smatg12. Expression of the EGFP-SmATG12 fusion construct complemented the sterile phenotype of $\Delta$ Smatg12. EGFP-SmATG12 localizes to the cytoplasm and at phagophore assembly sites indicated by small arrows and cup-shaped phagophores indicated by long arrows. Vacuolar membranes were stained using FM4-64. The merged picture shows a close up. (B) Localization of EGFPSmATG12 in the $\Delta$ Smatg8 mutant (Voigt and Pöggeler 2013a). The fluorescence signals are larger and more distinct than signals in the complemented mutant $\Delta$ Smatg8::egfp-Smatg8 $8^{\text {ect }}$ (compare to E). (C) The fluorescence signal of free EGFP in the wt strain transformed with plasmid p1783-1 served as control. (D) When the mutant strain $\Delta$ Smatg12 expressed EGFP-SmATG8 (plasmid pRS-egfp-Smatg8 (Voigt and Pöggeler 2013a)), the fluorescence protein displays an equal diffused signal in the cytoplasm with large accumulating spots (arrow head) which are excluded from the vacuole. Vacuolar membranes were co-stained with FM4-64 and pictures were merged. (E) The previously constructed strain $\Delta$ Smatg8::egfp-Smatg8 ${ }^{\text {ect }}$ (Voigt and Pöggeler 2013a) was used to compare the localization of EGFP-SmATG8 in the $\triangle$ Smatg12 mutant. Autophagosomes are indicated by arrows. DIC, differential interference contrast; EGFP, enhanced green fluorescence protein. Autophagy was induced by the addition of $2.5 \mathrm{mM} 3$-AT to the SWG medium or by nitrogen starvation conditions (SWG-N, without $\mathrm{KNO}_{3}$ and arginine). Scale bars as depicted. 
These microscopic observations correlate with the EGFP-SmATG8 degradation analysis by Western blotting (Figure 14). Vacuolar membranes were stained with the membrane dyes FM464. The localization did not change when histidine starvation was induced by 3-AT (Voigt and Pöggeler 2013a) or when strain grew under nitrogen-limiting conditions. Fluorescence microscopy revealed the expected localization of EGFP-SmATG8 in vacuoles and in autophagosome-like structures (distinct spots in the cytoplasm) as well as in basal hyphal compartments in the lumen of the vacuoles in the complemented $\Delta$ Smatg8 (Figure 16E) (Voigt and Pöggeler 2013a). These results indicated that macroautophagy was disrupted in the $\Delta$ Smatg12 mutant. 


\subsection{Identification of the selective autophagy cargo receptor SmNBR1}

\subsubsection{The cargo receptor SmNBR1 of $S$. macrospora is conserved in higher eukaryotes and displays a similar domain architecture}

In a study, to identify interacting proteins of the ubiquitin-like autophagy protein SmATG8 a GFP-Trap analysis was used followed by LC/MS, and identified among other proteins a protein that had sequence similarity to the mammalian selective autophagy cargo receptor NBR1 (Voigt 2012). The identified protein is encoded by the 2681-bp ORF SMAC_07844 and has 863 amino acids (aa) with a predicted molecular weight of $96 \mathrm{kDa}$. The splicing of a predicted single intron (92 bp) within the ORF was confirmed by sequencing the cDNA. A phylogenetic analysis was performed based on 20 NBR1 homologs of different species including filamentous ascomycetes, basidiomycetes, $H$. sapiens, A. thaliana and $S$. pompe. The unrooted neighbor joining tree demonstrates that NBR1 homologs from filamentous ascomycetes form a distinct clade (Figure 17).

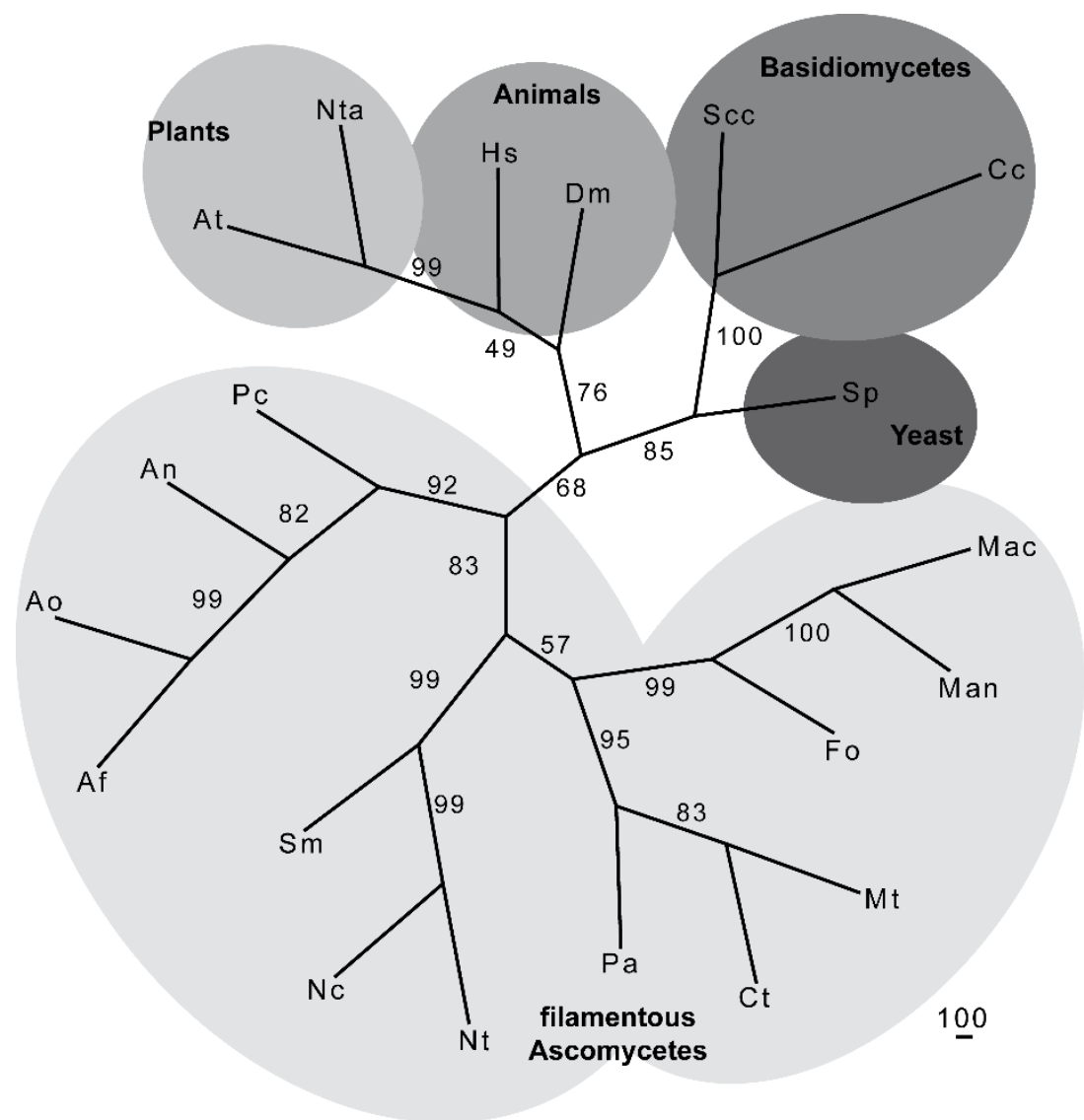

Figure 17: Unrooted phylogenetic neighbor-joining tree of NBR1 homologs from 20 species.

The phylogenetic relationship based on the alignment of the full-length NBR1 homologs. The number at the nodes indicate the percentage of bootstrap support based on 1000 bootstrap replications. Abbreviations and accession numbers of proteins are as follows: filamentous Ascomycetes: Sm, Sordaria macrospora (XP_003346367); Nc, Neurospora crassa (XP_011394647); Nt, Neurospora tetrasperma (XP_009847604); Pa, Podospora anserina (XP_001912058); Mt, Myceliophthora thermophile (MYCTH_2302632); Ct, Chaetomium thermophilum 
(XP_006696593); Man, Metarhizium anisopliae (EFY98453); Mac, Metarhizium acridum (EFY92904); Fo, Fusarium oxysporum (EMT63555); Af, Aspergillus fumigatus (XP_755022); Ao, Aspergillus oryzae (XP_001824430); An, Aspergillus nidulans (XP_661032); Pc, Penicillium chrysogenum (XP_002563338); Basidiomycetes: Scc, Schizophyllum commune (XP_003038660); Cc, Coprinopsis cinerea (XP_001829200); Plants: At, Arabidopsis thaliana (NP_194200); Nta, Nicotiana tabacum (NP_001312077); Animals: Hs, Homo sapiens (Q14596); Dm, Drosophila melanogaster (NP_648826); Yeast: Sp, Schizosaccharomyces pombe (Q9P792).

The identified ORF of $S$. macrospora revealed a conserved domain architecture, including an N-terminal PB1 domain followed by CC domains, four ZZ-type zinc-finger domains, a NBR1 box domain and an ATG8-interacting motif (LIR) (Figure 18, Supplement 3). Similar to other fungal NBR1 homologs, the S. macrospora protein SmNBR1 lacks the C-terminal UBA domain, which is found in the plant and mammalian homologs.

A
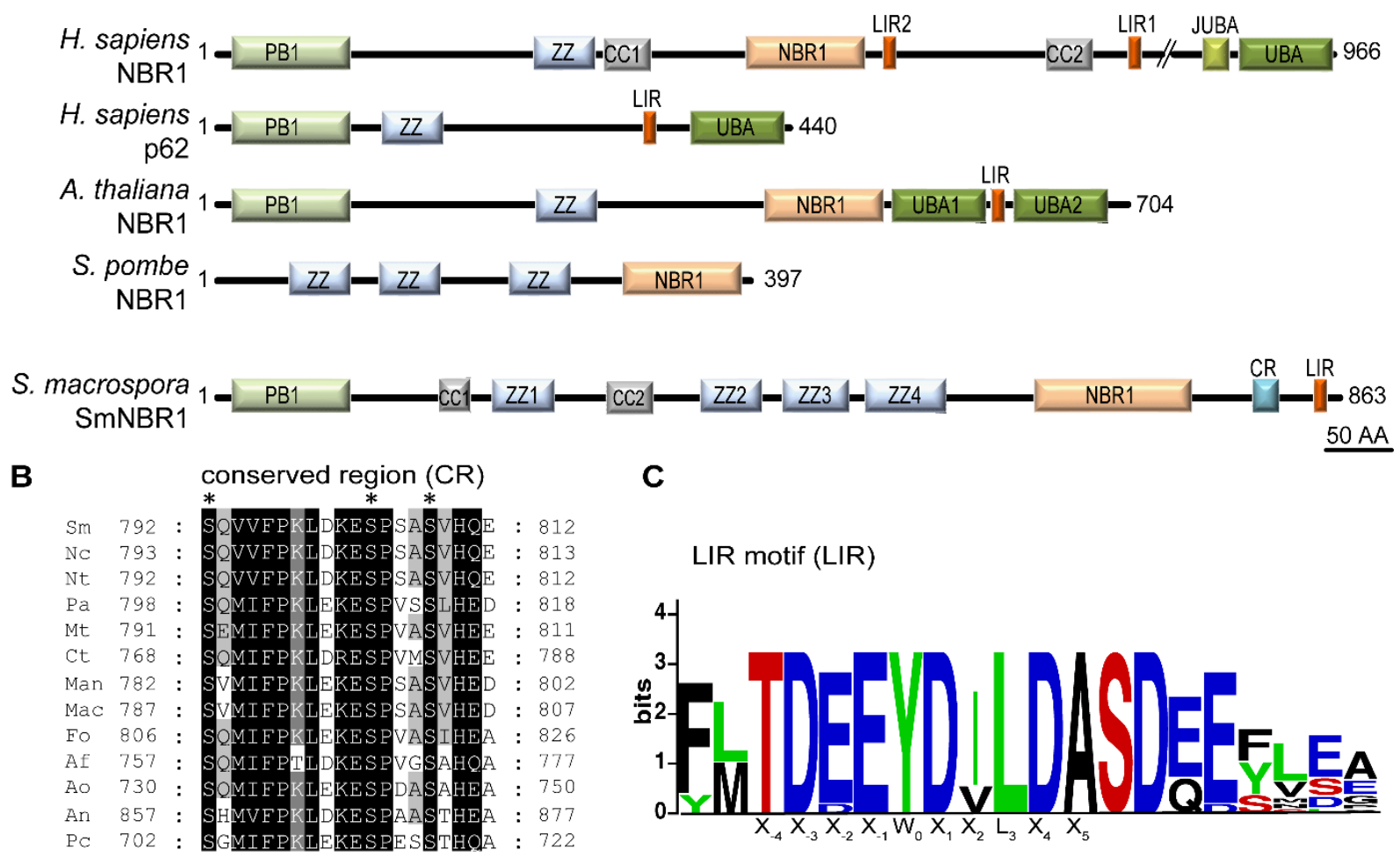

C

LIR motif (LIR)

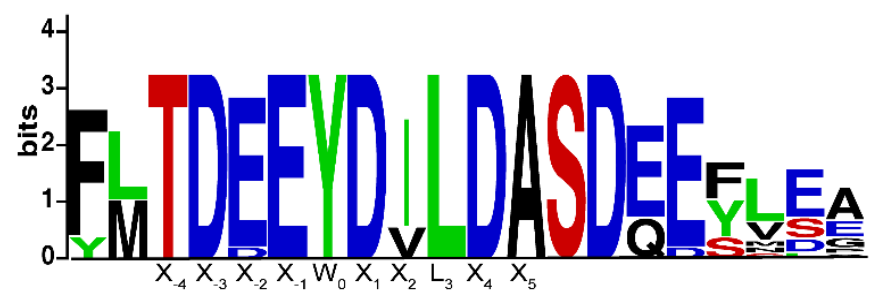

D

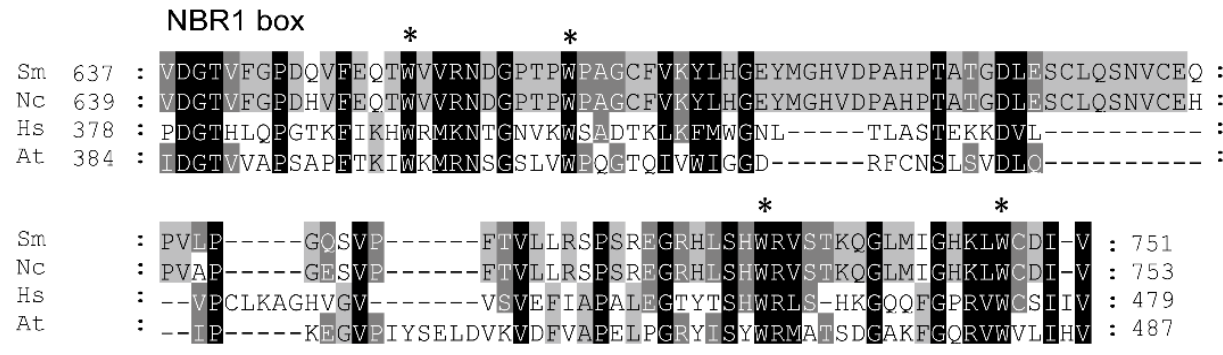

Figure 18: Schematic representation of cargo receptor homologs and specific domains.

(A) Protein architecture of NBR1 homologs of H. sapiens, A. thaliana, S. pombe and S. macrospora: PB1, Phox and $\underline{B} e m 1$ domain; ZZ, ZZ-type zinc-finger domain; CC, coiled-coil domain; NBR1-domain, LIR, LC3-interacting region; JUBA, juxta- ubiquitin-asssociated domain; UBA, ubiquitin-asssociated domain; CR, conserved region. Scale bar is indicated. (B) Alignment of 13 NBR1 homologs of filamentous ascomycetes revealed a fungal specific conserved region at the C-terminus. Sm, Sordaria macrospora (XP_003346367); Nc, Neurospora crassa 
(XP_011394647); Nt, Neurospora tetrasperma (XP_009847604); Pa, Podospora anserina (XP_001912058); Mt, Myceliophthora thermophile (MYCTH_2302632); Ct, Chaetomium thermophilum (XP_006696593); Man, Metarhizium anisopliae (EFY98453); Mac, Metarhizium acridum (EFY92904); Fo, Fusarium oxysporum (EMT63555); Af, Aspergillus fumigatus (XP_755022); Ao, Aspergillus oryzae (XP_001824430); An, Aspergillus nidulans (XP_661032); Pc, Penicillium chrysogenum (XP_002563338). Asterisks label the three conserved serine (S) residues. (C) Sequence logo was made using WebLogo 3 of the fungal NBR1 LIR motifs. Species as listed in B. (D) Alignment of the NBR1 box of the following species: Sm, Sordaria macrospora (XP_003346367); Nc, Neurospora crassa (XP_011394647); At, Arabidopsis thaliana (NP_194200); Hs, Homo sapiens (Q14596). Asterisks label the four conserved tryptophan $(\mathrm{W})$ residues typical for this domain.

Furthermore, in the alignment of NBR1 homologs a conserved region (CR) was identified near the NBR1 domain, which is characteristic for filamentous ascomycetes and less conserved in basidiomycetes (Figure 18B, Supplement 3A, including basidiomycetes), but not present in mammalian, plant or the fission yeast NBR1 receptors. Within this specific region, three serine (S) residues are conserved (Figure 18B). The amino-acid alignment from the filamentous ascomycetes identified also a sequence corresponding to the LIR consensus sequence $\left(\mathrm{X}_{-3}-\mathrm{X}_{-2}-\mathrm{X}_{-1}-\left(\mathrm{W} / \mathrm{Y} / \mathrm{F}_{0}\right)-\mathrm{X}_{1}-\mathrm{X}_{2}-\left(\mathrm{L} / \mathrm{I} / \mathrm{V}_{3}\right)-\mathrm{X}_{4}-\mathrm{X}_{5}\right)$ (Alemu et al. 2012) at the $\mathrm{C}$-terminus of SmNBR1 and fungal NBR1 homologs. All of them have an aromatic (Y) and a hydrophobic (L) residue at position $\mathrm{W}_{0}$ and $\mathrm{L}_{3}$, respectively. Within this region $\mathrm{X}_{-1}$ to $\mathrm{X}_{-3}$ amino acids are acidic residues (E, D) and $\mathrm{X}_{-4}$ is a conserved threonine (T) (Figure 18C, Supplement 3B, including basidiomycetes). The SmNBR1 protein harbors a NBR1 box with four conserved tryptophan residues (Figure 18D). Despite these conserved features, fungal NBR1 homologs lack the UBA-domain. A carefull inspection of the protein sequence did not identify a UBA domain.

\subsubsection{The Smnbr1 deletion mutant displays developmental defects}

To elucidate the function of SmNBR1, a S. macrospora Smnbrl deletion mutant was generated by homologous recombination with a $h p h$-deletion cassette flanked by upstream and downstream sequences of the Smnbrl ORF (2.2.6.1). The deletion of Smnbrl was confirmed by PCR and Southern blot analysis (Figure 19). Macroscopic and microscopic investigations of the $\Delta$ Smnbr1 strain revealed several developmental defects in comparison to the wild type and to a complemented strain expressing a Smnbrl-egfp fusion gene ectopically ( $\Delta$ Smnbr1::Smnbr1-egfp ${ }^{\text {ect }}$ ) (Figure 20). S. macrospora wild type and the complementation strain completed the life cycle within seven days under laboratory conditions. It starts with a germinating ascospore developing into a haploid vegetative mycelium. After three days, sexual development begins with the formation of female gametangia (ascogonia) which are protected by sterile hyphae that develop into a spherical fruiting-body precursor (protoperithecia). Cell 
differentiation leads to pigmentation after 5 days followed by development of the mature fruiting bodies (perithecium). During perithecia development after karyogamy, meiosis and a postmeiotic mitosis takes place, giving rise to eight linearly-ordered black ascospores per ascus (approximately 200 asci per perithecium). The ascospores are discharged through an apical pore (ostiole) at the neck of the fruiting body (Teichert et al. 2014). Microscopic analysis of the sexual reproductive structures of the $\Delta S$ Smbr1 strain showed defects at late stages of fruitingbody development. The mutant formed ascogonia, protoperithecia and perithecia but in a reduced number and after a prolonged time (Figure 20A+B).

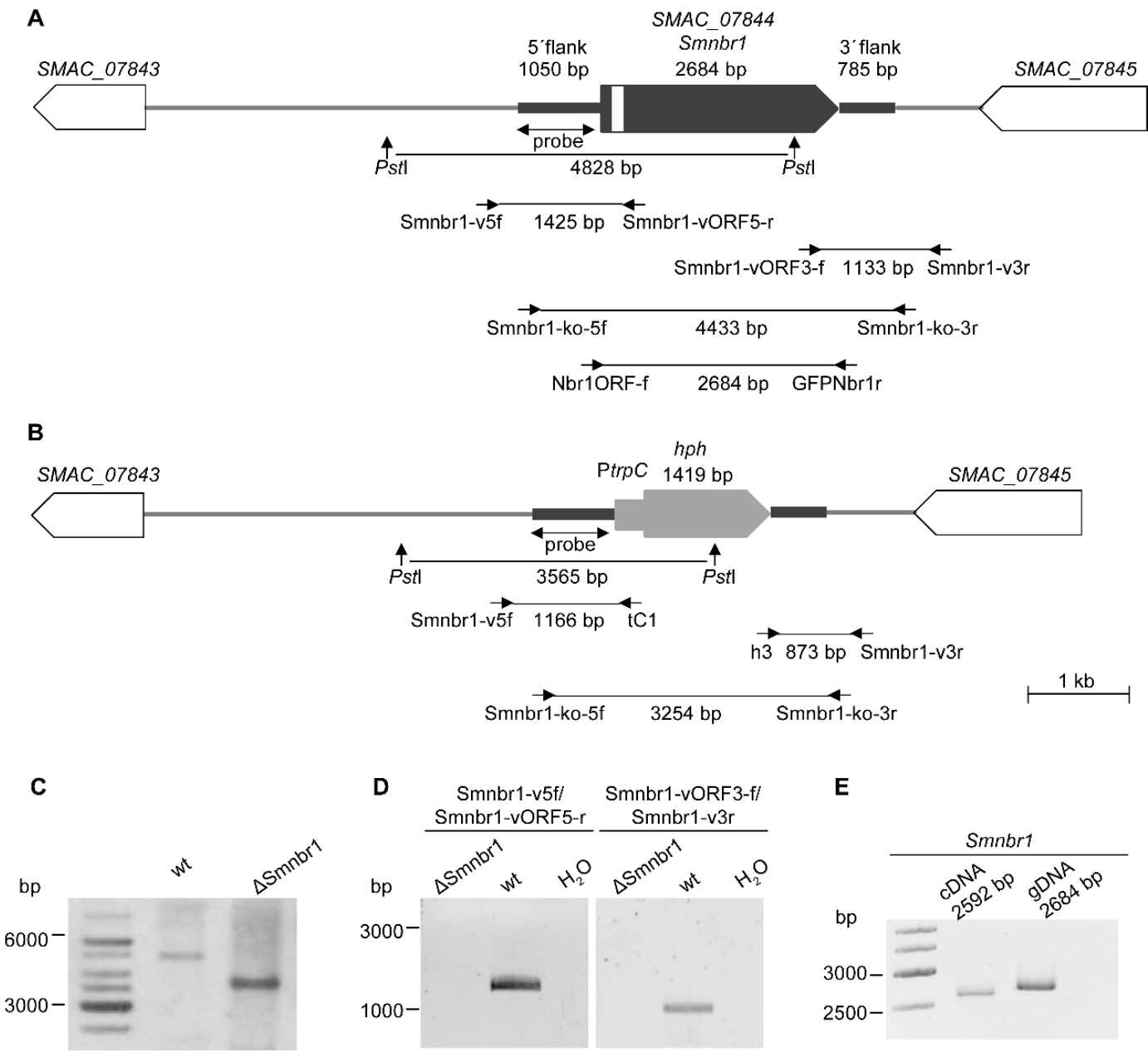

Figure 19: Generation and analysis of a $\triangle$ Smnbr1 knockout strain.

(A) Schematic representation of the Smnbrl (SMAC_07844, dark grey arrow) genomic region and its flanking regions including adjacent genes SMAC_07843 and SMAC_07845 (white arrows). The verified intron is indicated with a white bar. (B) Gene locus after homologous recombination of the $h p h$-resistance gene under the control of the $\operatorname{trpC}$ promoter of A. nidulans (PtrpC and $h p h$, grey arrow). The positions of primers to construct and verify homologous recombination at the Smnbrl locus are indicated as small black arrows. PstI restriction sites are labeled. (C) Southern blot analysis of wt and $\Delta$ Smnbr1 strain. Genomic DNA was digested with PstI and hybridized with the probe indicated in A and B. Hybridization resulted in a 4828-bp band for the wt and a 3565bp band for the knockout strain. (D) PCR analysis with primer pairs Smnbr1-v5f/Smnbr1-vORF5-r (1425 bp) and Smnbr1-vORF3-f/Smnbr1-v3r (1133 bp) to verify the absence of the Smnbrl gene in $\Delta$ Smnbr1 and the presence in wt. The presence of the $h p h$-resistance cassette at the desired gene locus was verified with primer pairs Smnbr1v5f/tC1 (1166 bp) and h3/Smnbr1-v3r (873 bp) (PCR not shown). Additionally, the primer pair Smnbr1-ko$5 \mathrm{f} / \mathrm{Smnbr} 1-\mathrm{ko}-3 \mathrm{r}$ was used to verify the presence of the $h p h$ gene (3254 bp) in comparison to wt (4433 bp) (PCR 
not shown). Position of the primers are indicated in (A) and (B). (E) PCR analysis of the cDNA (2592 bp) and gDNA (2684 bp) of Smnbrl which showed a bandshift induced by the splicing of the 92-bp intron. The primer pair Nbr1ORF-f/GFPNbr1r was used indicated in (A).

The ascus rosettes of the mutant harbored incomplete asci with few mature, melanized ascospores (Figure 20A,C,D). In the complemented strain these defects are partially rescued. Quantitative analysis of fruiting bodies showed a $97.5 \%$ reduction of mature perithecia per $\mathrm{cm}^{2}$ in the knockout strain in comparison to the wild type (Figure 20B). Consequently, the number of discharged mature ascospores, which are essential for reproduction, was also drastically reduced (99\% reduction in comparison to wild type) (Figure 20C). 

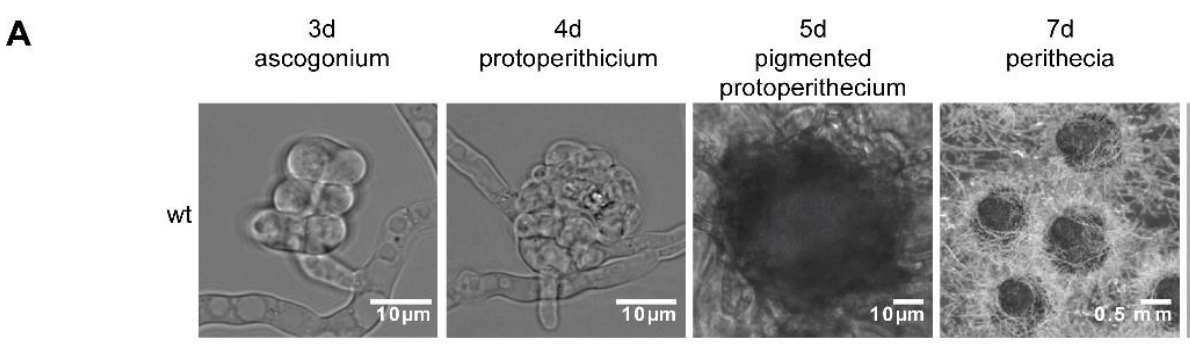

$8 d$

ascospores

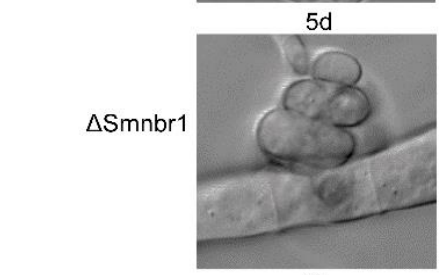

$6 d$

$7 d$

$9 d$

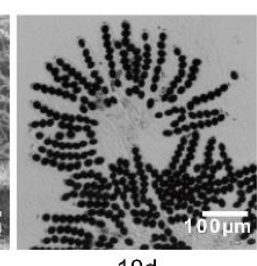

$10 \mathrm{~d}$
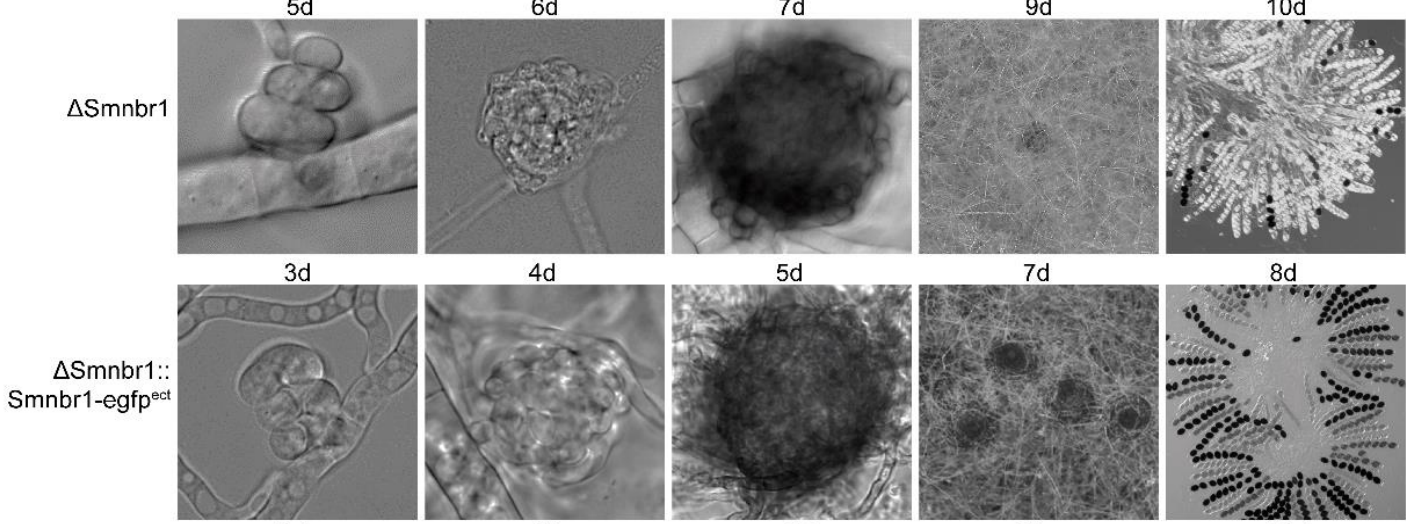

$4 d$
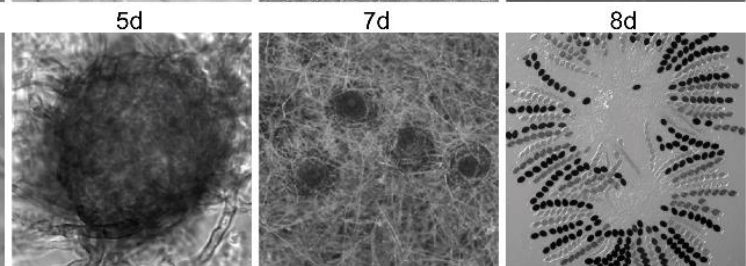

$3 d$

$4 d$

$5 d$
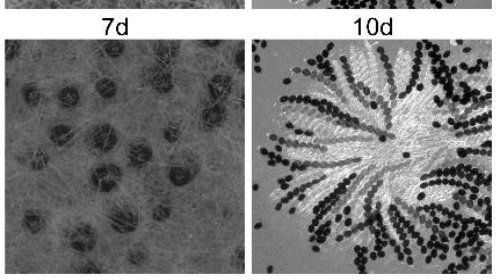

$\triangle S m n b r 1$. Hsnbr1-Dsredec
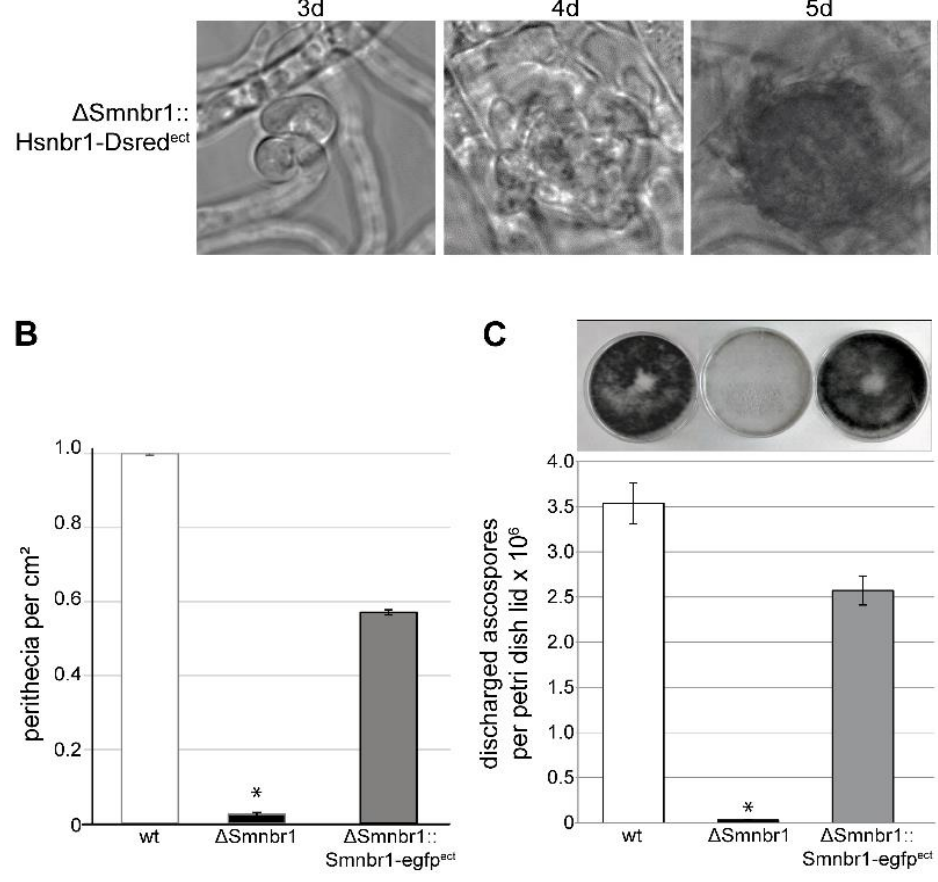

C

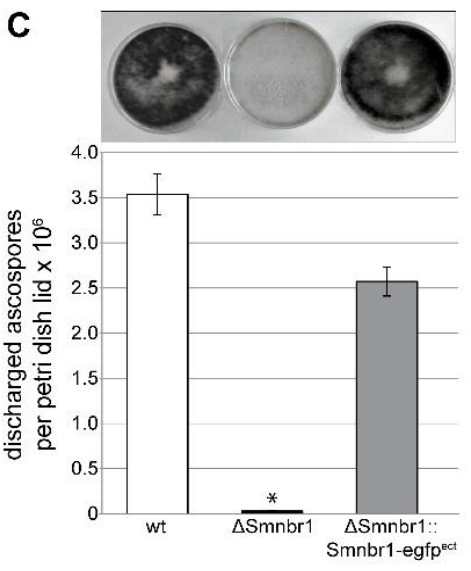

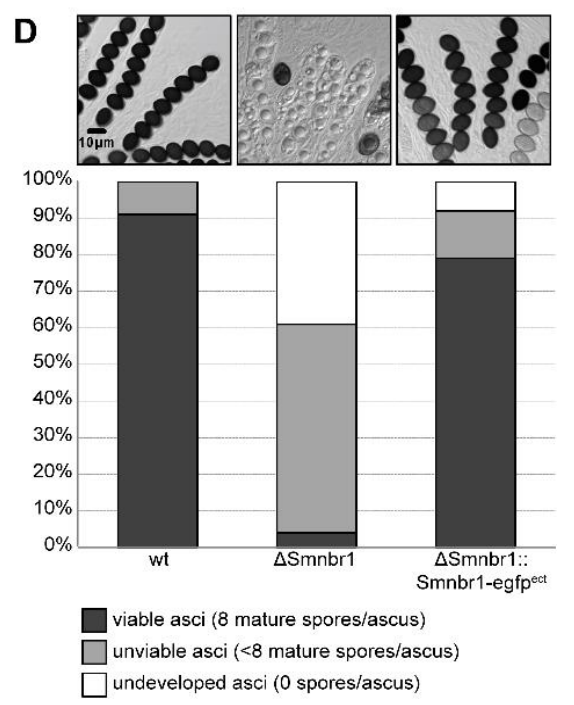

Figure 20: Phenotypic analysis of $S$. macrospora $w t, \Delta$ Smnbr1 mutant strain and complementation strains expressing $S$. macrospora or $H$. sapiens NBR1 proteins.

(A) Microscopic investigation of sexual development of $\Delta \mathrm{Smnbr} 1$ compared to wt and the complemented strains

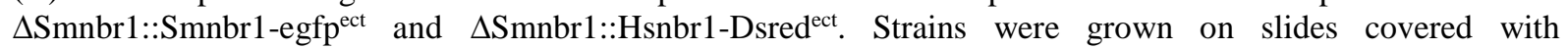
fructification medium (SWG), and photographs were taken at indicated days. (B) The perithecia were counted per $\mathrm{cm}^{2}$ after eight days (wt, $\left.\Delta \mathrm{Smnbr} 1:: \mathrm{Smnbr1-egfp}{ }^{\text {ect }}\right)$ or ten days $(\Delta \mathrm{Smnbr} 1)$. Number of perithecia shown are averages from nine independent measurements of three independent experiments $(n=27)$. (C) Discharged ascospores, after ten days of growth, from the lid of a petri dish $\left(56.7 \mathrm{~cm}^{2}\right)$ for the different strains. (D) Ascus rosettes were analyzed at the same time point as in (B). Microscopic pictures represent a part of representive ascus rosettes. All strains were grown on fructification medium (SWG). Wt was set to 1. Results of (C) and (D) are averages from 25 independent measurements of four independent experiments $(\mathrm{n}=100)$. Standard deviations are indicated by error bars (B) and (C) and scale bars are shown in the images. (*) Asterisks indicate significant differences in the deletion mutant in comparison to the wt according to Student's t-test ( $\mathrm{p}<0.0000001)$. 
Microscopic analysis showed that the mature perithecia of the knockout strain contained a high number of undeveloped asci and unviable ascospores in comparison to the wild type (Figure 20D). Thus, we showed that the deletion of Smnbrl decreased the production of mature perithecia, with an increased number of defective asci and ascospores.

The SmNBR1 homolog shares only $22 \%$ identity to the NBR1 protein from H. sapiens. However, many domains of the H. sapiens NBR1 protein (HsNBR1) are conserved in SmNBR1. Therefore, we analyzed whether the HsNBR1 protein can complement the $\Delta$ Smnbr1 phenotype by expressing the protein in the $\Delta$ Smnbr1 strain. For this, $\Delta$ Smnbr1 was transformed with the plasmid pHsnbr1-Dsred. Subsequently, $20 \Delta$ Smnbr1::Hsnbr1-Dsred ${ }^{\text {ect }}$ transformants were phenotypically analyzed. After ten days growth on SWG medium 14 of 20 transformants show complementation by producing mature perithecia and ascospores as the $S$. macrospora complemenation strain. The six other transformants were not able to rescue the phenotypical defects of $\Delta$ Smnbr1 perhaps due to the ectopic integration of the heterologous gene (data not shown). Three of the 14 complementation strains expressing the HsNBR1 protein were tested phenotypically in more detail. Strains were able to form ascogonia, protoperithecia and perithecia. The maturation of the late developmental stages are more delayed in comparison to the wild type because the pigmented protoperithecia and perithecia looked smaller. In contrast to the mutant $\Delta$ Smnbr1, the $H$. sapiens complementation strain shows more mature ascospores in ascus rosettes after ten days of growth. In summary, the developmental defects of the mutant $\Delta$ Smnbr1 can be partially rescued by the human NBR1 homolog (Figure 20A). The heterologous production of HsNBR1-DsRED was verified by a Western-blot experiment (Supplement 4).

Due to the fact that SmNBR1 lacks an obvious UBA domain we tested the enrichment of ubiquitinated proteins in total protein extracts of wild type, $\Delta$ Smnbrl (two independent mutants) and a control strain $\Delta$ Smubp3 (Herzog pers. comm.). The deubiquitination enzyme SmUBP3 is able to bind ubiquitinated proteins to remove the ubiquitin tag for their autophagosomal degradation (Kraft et al. 2008). The Western-blot analysis showed that the $\Delta$ Smnbr1 strains behave like the wild type strain whereas the $\Delta$ Smubp3 strain showed a high accumulation of ubiquitinated proteins, however several bands seem to be more intensive in the $\Delta$ Smnbr1 strain as in the wild type (Figure 21 ). 


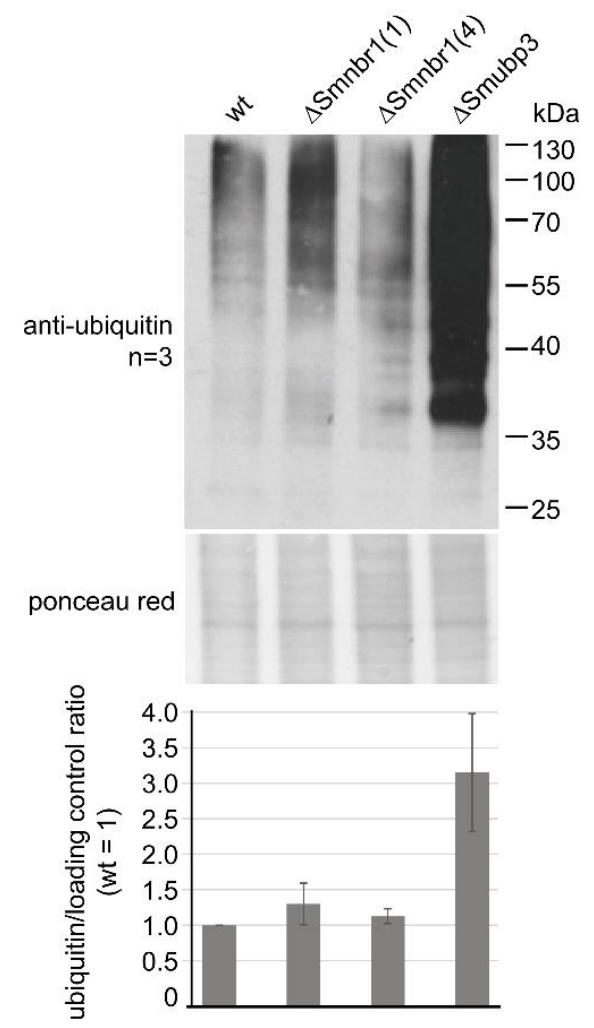

Figure 21: Western blot using anti-ubiquitin to detect ubiquitinated proteins.

Western blot using equal amounts of protein extracts of wt, $\Delta \mathrm{Smnbr} 1$ and $\Delta \mathrm{Smubp} 3$ (control) strain. Ubiquitinated proteins were detected with an ubiquitin antibody. Detection of actin was used as loading control to show the protein levels. One of three Western-blot experiments is represented. Quantification of Western blots was done using the program ImageJ. For this the ubiquitin protein amount was quantified to the actin loading control. Wt was set to $100 \%$. Data in this diagram are represented for three independent experiments. Error bars are indicated.

\subsubsection{Stress conditions enhance developmental defects of $\Delta$ Smnbr1}

Autophagy enables cells to adapt to new conditions or to overcome starvation or environmental stress conditions. Therefore, we tested whether deletion of Smnbrl leads to growth and adaptation defects under different stress conditions. For this the growth rate was measured using race tubes (2.2.1). The vegetative growth rate of the deletion strain was only slightly reduced to $2.6 \mathrm{~cm} / \mathrm{d}$ compared to $3.1 \mathrm{~cm} / \mathrm{d}$ of the wild type strain under normal growth conditions (SWG medium). In contrast, under histidine-starvation conditions induced by the drug 3-amino-1,2,4triazole (3-AT) or nitrogen starvation by omitting $\mathrm{KNO}_{3}$ /arginine in the $\mathrm{SWG}$ medium the $\Delta$ Smnbr1 mutant showed a significant reduction of the growth rate in comparison to the wild type (Figure 22A). To test the sensitivity of the $\Delta$ Smnbr1 strain to other stress conditions we induced different kinds of stresses, such as amino-acid starvation, osmotic-, oxidative-, or cell wall stress conditions by adding to the SWG medium $2.5 \mathrm{mM}$ 3-AT, $0.5 \mathrm{M}$ sorbitol, $0.25 \mathrm{mM}$ $\mathrm{NaCl}, 0.02 \% \mathrm{H}_{2} \mathrm{O}_{2}$ or $0.005 \%$ SDS, respectively. Additionally, nitrogen starvation was performed by limiting the nitrogen sources $\mathrm{KNO}_{3}$, arginine or biotin and temperature stress was induced by cultivating the strains at $20^{\circ} \mathrm{C}$ or $32^{\circ} \mathrm{C}$ (Figure 22B). 


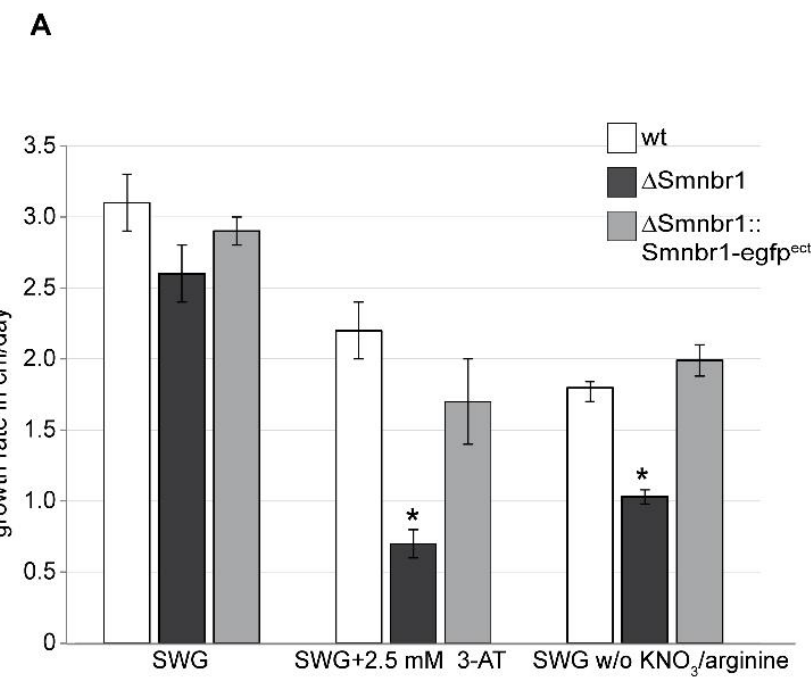

Figure 22: Vegetative growth and development analysis of $S$. macrospora wt, $\Delta S \operatorname{Smbr} 1$ and the complemented mutant on different medium.

(A) The growth rate of wt, $\Delta$ Smnbr1 and complemented strain was analyzed by measuring the growth rate in $\mathrm{cm} /$ day. Growth-rate analysis was conducted in $30-\mathrm{cm}$ race tubes filled with SWG, SWG+ $2.5 \mathrm{mM}$ 3-AT or SWG without (w/o) $\mathrm{KNO}_{3} /$ arginine. Results are averages of three biological replicates performed of seven independent samples $(\mathrm{n}=21)$ and standard deviations are indicated by error bars. Values labeled with an asterisk are significantly different from wt growth velocity according to Student's t-test $(\mathrm{p}<0.0000001)$. (B) Strains from (A) were grown in presence of different stress conditions, such as amino-acid starvation (2.5 $\mathrm{mM} 3$-AT), osmotic- (0.5 M sorbitol, $0.25 \mathrm{mM} \mathrm{NaCl})$, oxidative- $\left(0.02 \% \mathrm{H}_{2} \mathrm{O}_{2}\right)$ or cell wall stress $(0.005 \%$ SDS) by adding the components to SWG medium. Limitation of nitrogen sources was performed by omitting $\mathrm{KNO}_{3}$, arginine or biotin and temperature stress was performed at $20^{\circ} \mathrm{C}$ or $32^{\circ} \mathrm{C}$. Pictures of cultivated agar plates were taken after $8 \mathrm{~d}$ as well as the microscopic sections. Scale bars are indicated.

B

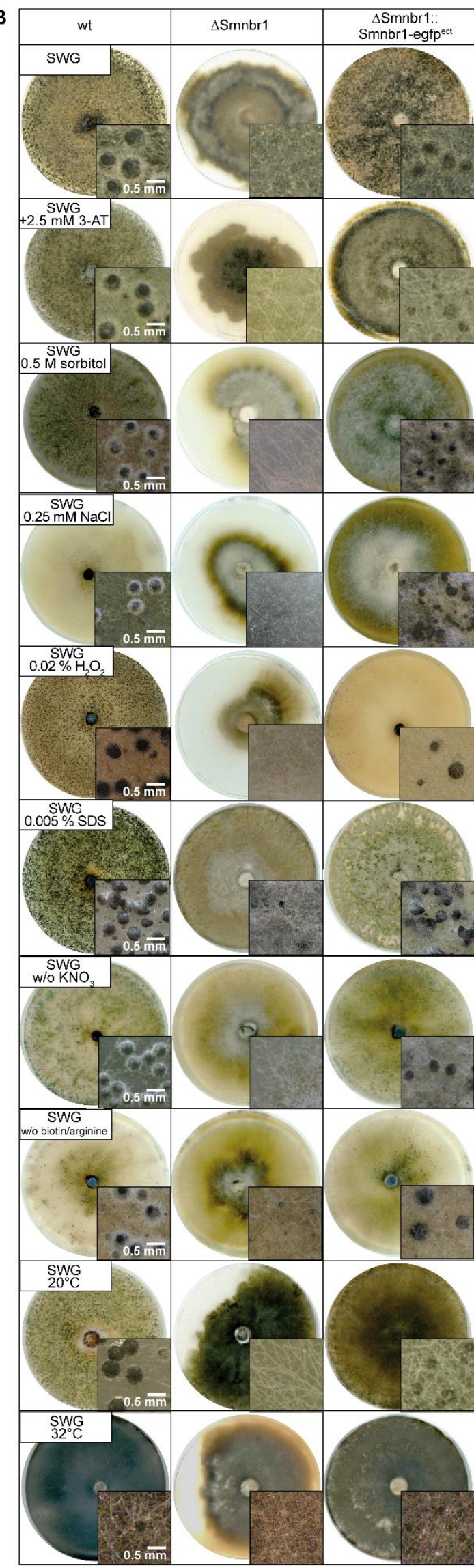


While the wild type and the complemented strain displayed normal vegetative growth under all conditions tested, the $\Delta$ Smnbrl knockout strain showed defects under different stress conditions. Mostly, the mutant was unable to cover the whole petri dish and often it terminates the growth, such as observed under osmotic stress (sorbitol, $\mathrm{NaCl}$ ), starvation conditions (3AT) and temperature stress. Nitrogen starvation and oxidative stress inhibited fruiting-body development of the knockout strain characterized by production of little or no protoperithecia or perithecia.

The fact that developmental defects of the $\Delta$ Smnbrl can be almost completely rescued by complementation of the deletion strain with the wild type Smnbr1 gene ( $\Delta$ Smnbr1::Smnbr1$\operatorname{egfp}^{\text {ect }}$ ), verifies that the developmental phenotype results from the lack of the SmNBR1 function (Figure 22). Taken together, phenotypical characterization under stress conditions revealed that SmNBR1 is necessary for proper fruiting-body development and vegetative growth and required to overcome nutrient limiting and stress conditions.

\subsubsection{SmATG8 interacts directly with SmNBR1}

In mammals and plants it is known that NBR1 and ATG8 interact with each other in a LIRdependent manner (Johansen and Lamark 2011). Co-immunoprecipitation (Co-IP) experiments with $S$. macrospora wild type strain expressing a DsRED-tagged version of SmNBR1 together with an EGFP-tagged version of SmATG8 confirmed the interaction between both proteins in vivo (Figure 23A).

To validate the interaction of SmATG8 and SmNBR1, and to specify the interacting domains, we performed a yeast-two hybrid $(\mathrm{Y} 2 \mathrm{H})$ analysis with the full-length protein and different truncated or mutated versions of SmNBR1. For this purpose, Smnbrl and Smatg8 cDNAs were cloned into the two-hybrid vectors pGBKT7 (bait) and pGADT7 (prey), respectively, either harbouring the Gal4-binding or Gal4-activation domain. We observed that the full-length protein SmNBR1 can interact with SmATG8 when SmNBR1 is fused to the Gal4-activation domain and SmATG8 is fused to the Gal4-binding domain. No interaction was observed when SmNBR1 was fused to the binding domain and SmATG8 to the activation domain while the positive control pAD-ranBPM in combination with pBD-Smnbr1 was functional. All controls for self-activation were tested negatively (data not shown). Therefore, we coupled all the different versions of SmNBR1 to the Gal4-activation domain (Figure 23B,C). 
A

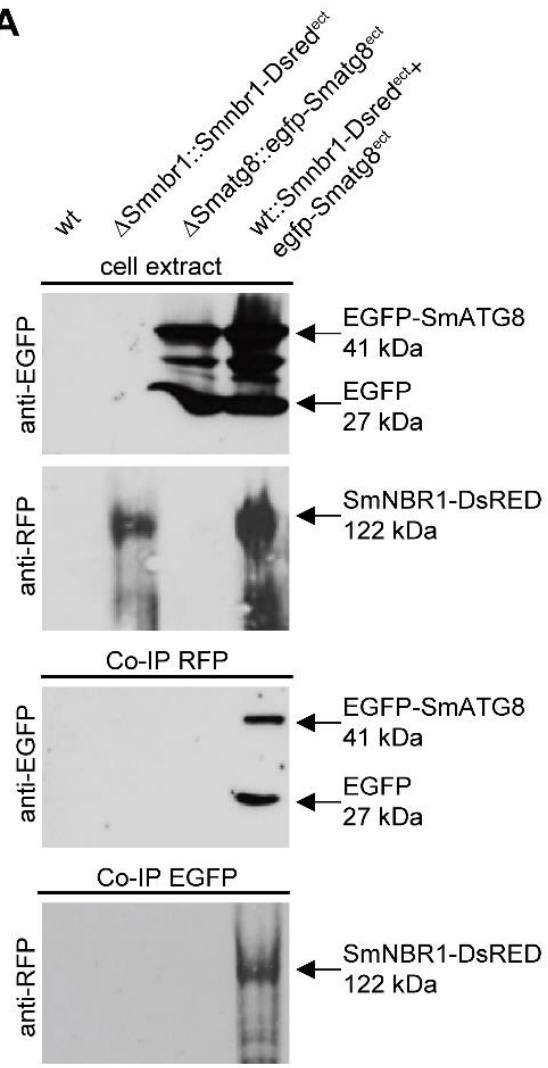

B schematic representation of AD-Smnbr1 versions

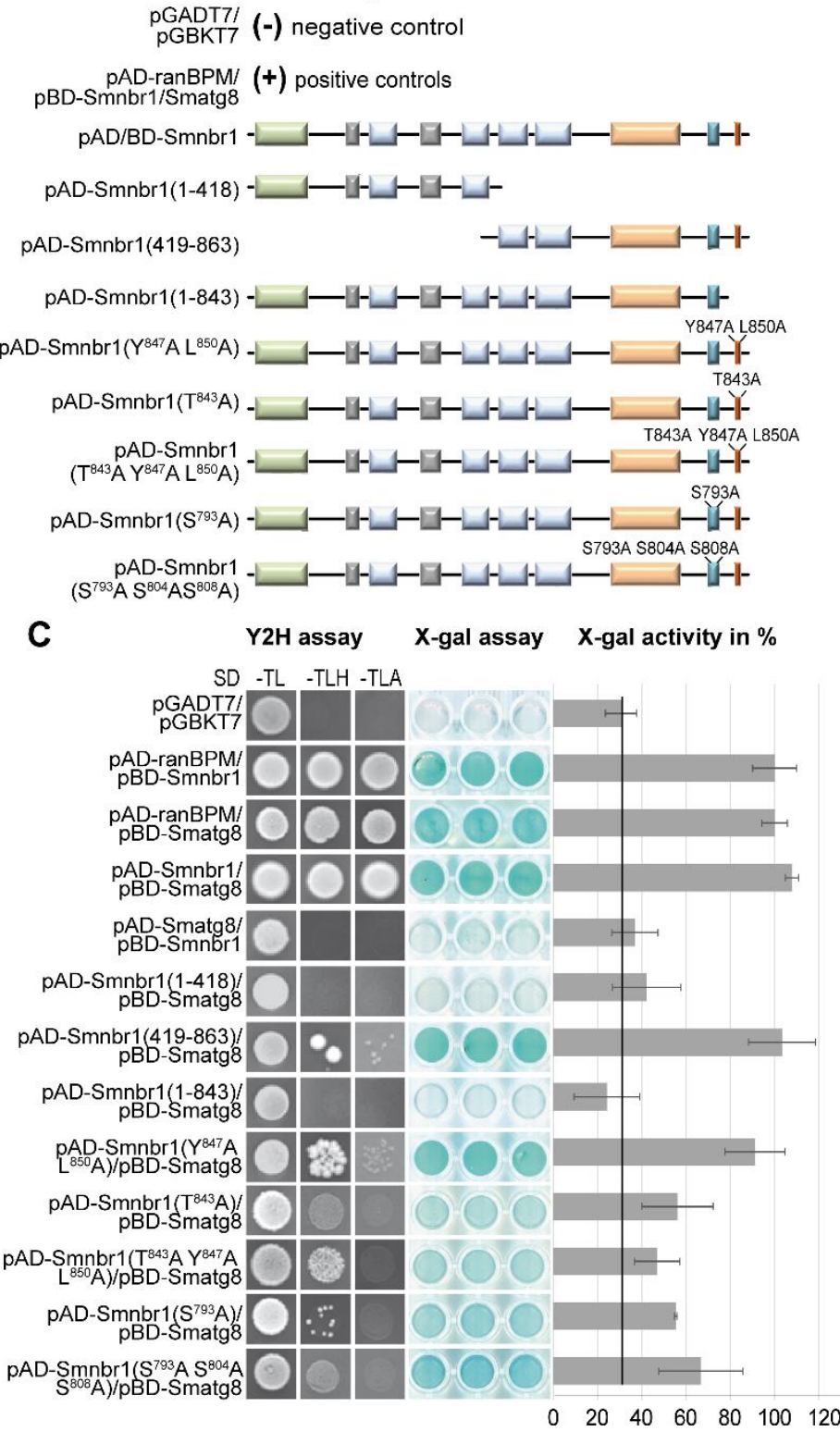

Figure 23: Interaction studies with SmNBR1 and SmATG8.

(A) Co-IP of SmNBR1 and SmATG8 and subsequent Western-blot analysis with anti-EGFP and anti-RFP antibodies from indicated strains. Expression of an EGFP-SmATG8 and SmNBR1-DsRED fusion construct in the wt strain. The respective complementation strains ( $\Delta$ Smatg8::egfp-Smatg8 ${ }^{\text {ect }}, \Delta$ Smnbr1::Smnbr1-Dsred $\left.{ }^{\text {ect }}\right)$ and wt strains were used as controls. (B) Schematic display of the domain architecture of different yeast-two hybrid Smnbrl constructs. (C) Yeast-two hybrid analysis: yeast strains expressing the fusion constructs AD-SmATG8 (activation domain of the Gal4-transcription factor encoded by pGADT7) and BD-SmNBR1 (binding domain of the Gal4-transcription factor encoded by pGBKT7) are not able to interact in contrast to the fusion of AD-SmNBR1 and BD-SmATG8. All mutated versions of AD-SmNBR1 and the C-terminal region (419-863) show a reduced interaction with BD-SmATG8. The other C-terminally truncated versions of SmNBR1 (1-418; 1-843) fused to the activation domain, do not interact with BD-SmATG8. The mated yeast cells were grown on SD minimal mediumtrp and -leu (SD-TL) for selection of the plasmids. SD media lacking -trp, -leu, -his/-ade (SD-TLH/A) were used for interaction studies. Each drop assay was performed in 10-fold serial dilutions $\left(10^{-1}-10^{-4}\right)$. Here, the growth of the first dilution is shown. As a negative control, the query vectors pGADT7/pGBKT7, and for positive control the $\mathrm{pAD}$-ranBpm/pBD-Smnbr1 and pBD-Smatg8 were used. RanBpm can used as positive control because it is able interact with the Gal4-BD (Tucker et al. 2009). To quantify the interaction of SmNBR1 and SmATG8 with the lacZ-reporter gene, the X-Gal activity was measured based on a PXG assay. The results are also represented in a bar diagram based on averages from 3-6 independent experiments of four identical measurements. The black line depicts the threshold based on the negative control. Error bars are indicated in the diagram. 
The full-length protein SmNBR1 is able to interact with SmATG8 confirming the results of the Co-IP analysis. To define the interaction domain of both proteins more closely, truncated versions of SmNBR1 were constructed consisting of either the N-terminal part (aa 1-418), the C-terminal part (aa 419-863) or a truncated version without the LIR motif (SmNBR1 1-843) and tested for interaction with SmATG8. Interestingly, only the C-terminal part of SmNBR1 containing the LIR and the CR motif was able to interact with SmATG8 (Figure 23B,C).

Additionally, mutated SmNBR1 variants were analyzed in which Tyr 843, Thr 847 and/or Leu 850 residues in the conserved TxxxYxxL LIR motif and Ser 793, 804, 808 in the CR motif were exchanged by Ala. When the LIR motif was deleted or mutated ( $\triangle$ aa $842-863$ of SmNBR1; $\mathrm{Y}^{847} \mathrm{~A} \mathrm{~L}{ }^{850} \mathrm{~A} ; \mathrm{T}^{843} \mathrm{~A} ; \mathrm{T}^{843} \mathrm{~A} \mathrm{Y}^{847} \mathrm{~A} \mathrm{~L}^{850} \mathrm{~A}$ ) interaction was abolished or impaired, as was the case for truncated versions without LIR motif. This indicates that the LIR motif is required for interaction with SmATG8. We tested also the impact of the CR motif for interaction with SmATG8. Mutation of the $\mathrm{S}^{793} \mathrm{~A} \mathrm{~S}^{804} \mathrm{~A} \mathrm{~S}^{808} \mathrm{~A}$ revealed also a reduced interaction ability.

\subsubsection{SmNBR1 localizes in autophagosome-like structures and vacuoles}

To determine the cellular localization of SmNBR1, we performed fluorescence microscopy using functionally expressed SmNBR1-DsRED or SmNBR1-EGFP. For this purpose, we used the complementation strains $\Delta$ Smnbr1::Smnbr1-Dsred ${ }^{\text {ect }}$ and $\Delta$ Smnbr1::Smnbr1-egfp ${ }^{\text {ect }}$ harboring the plasmids pSmnbr1-Dsred or pSmnbr1-egfp encoding either C-terminally tagged Smnbrl-Dsred or Smnbrl-egfp fusion genes under the control of the native promoter (SmNBR1-EGFP is shown in Supplement 5). As a localization control, fluorescence microscopy was performed with wild type transformants expressing only DsRED from plasmid pRHN1, and EGFP from plasmid p1783-1. When DsRED or EGFP were expressed individually, both proteins were found to be located in the cytoplasm in an even distribution (Figure 24B). SmNBR1 tagged with either EGFP or DsRED is localized as distinct spots in the cytoplasm, presumably representing autophagosomes, and is transported to the vacuole, indicating vacuolar degradation of the tagged protein. Additionally, the wild type strain was cotransformed with plasmids pSmnbr1-Dsred and pRS-egfp-Smatg8 which encodes EGFP fused N-terminally to SmATG8 under the control of its native promoter. The merged images show co-localization of EGFP-SmATG8 and SmNBR1-DsRED in cytosolic autophagosome-like structure and in vacuoles of young and old hyphae (Figure 24A). 
A

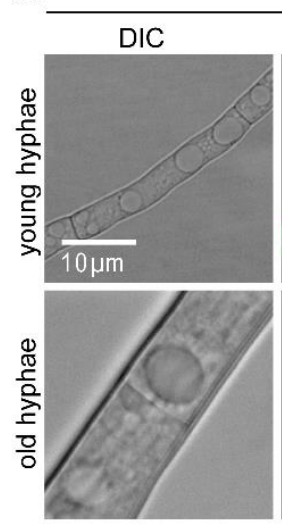

B

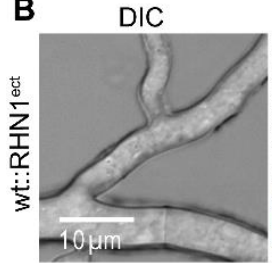

DIC

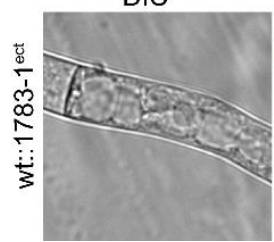

wt::Smnbr1-Dsred ${ }^{\text {ect }}+$ egfp-Smatg $8^{\text {ect }}$
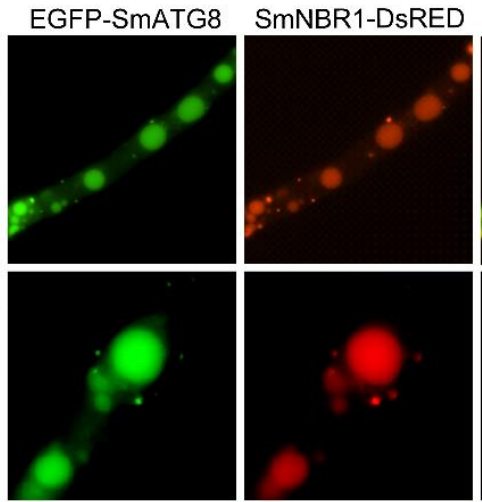

DSRED

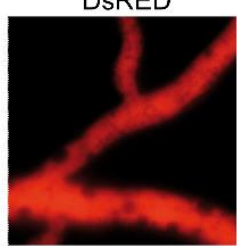

EGFP

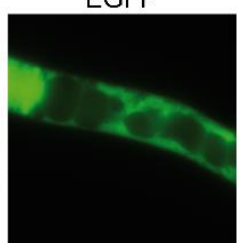

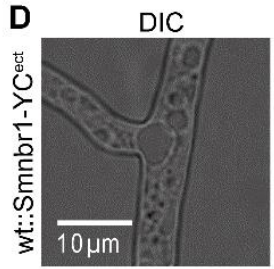

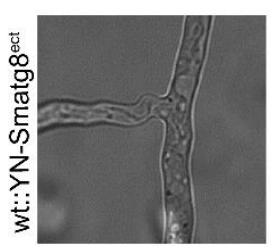

MERGE

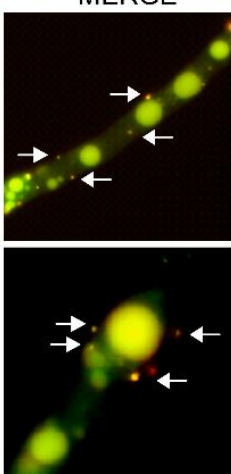

YFP
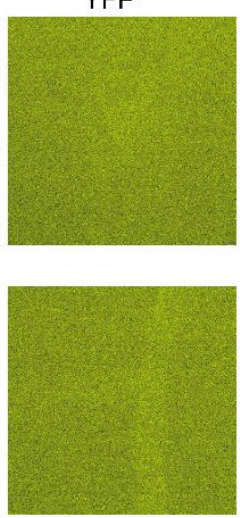

C wt::Smnbr1-YC ${ }^{\text {ect }}+Y N-S m a t g 8^{\text {ect }}$
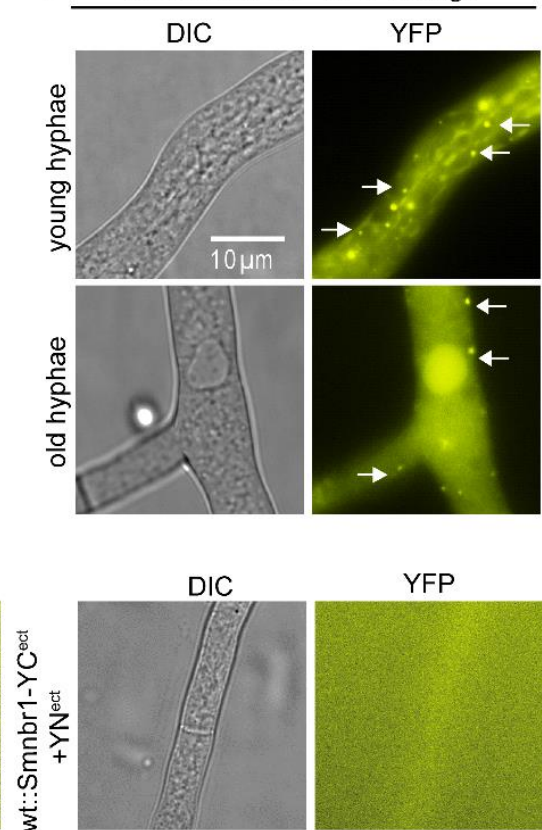

YFP
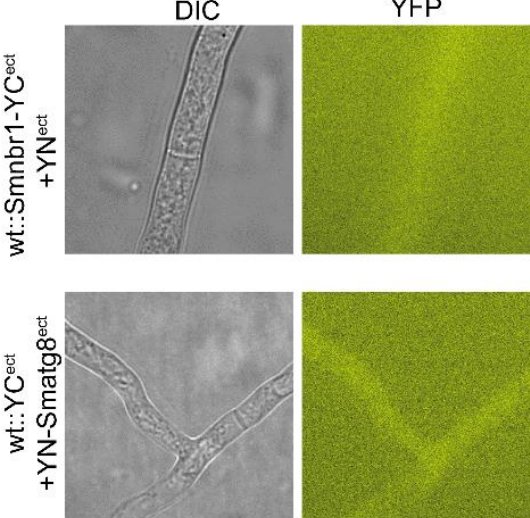

Figure 24: Localization of the autophagy proteins SmNBR1 and SmATG8.

(A) Fluorescence microscopic analysis of an S. macrospora wt strain carrying the plasmids pSmnbr1-Dsred and pRS-egfp-Smatg8 expressing a SmNBR1-DsRED and EGFP-SmATG8 fusion construct, respectively. At the hyphal tips EGFP-SmATG8 fluorescence is distributed in punctured autophagosome-like structures and larger aggregates in direct contact with SmNBR1-DsRED. In older basal hyphae, both proteins are concentrated in the vacuoles. Co-localization of SmNBR1 and SmATG8 is shown in yellow in the merged pictures. Arrows point to autophagosomes. (B) Fluorescence microscopy of wt strains encoding EGFP or DsRED (wt::1783-1 $\left.w t:: R H N 1^{\text {ett }}\right)$ to analyze the fluorescence signal of free EGFP and DsRED. Both signals are distributed in the cytoplasm. (C) BiFC experiment using a wt strain harboring the plasmids pSmnbr1-YC and pYN-Smatg8. The interaction of SmNBR1-YC and YN-SmATG8 is indicated by a yellow fluorescence signal (YFP). Arrows point to autophagosomes. (D) Fluorescence control strains for the BiFC assay. Wt transformed with vectors pSmnbr1YC and pYN-Smatg8 in single or co-transformed with the empty vectors pRS-YC and pRS-YN. All controls cannot emit the excitation light coming from YN or YC. DIC, differential interference contrast. Scale bars are indicated.

In order to verify the interaction of SmNBR1 and SmATG8 by another independent method, we performed a bimolecular fluorescence complementation (BiFC) assay, which is based on the split yellow fluorescent protein (YFP) (Hu and Kerppola 2003). YFP is divided in an Nterminal and a C-terminal part and both non-fluorescent fragments (YN and YC) were fused to putative interacting proteins SmATG8 and SmNBR1, respectively. Plasmids were transformed in the $S$. macrospora wild type strain. The association of the interaction partners allows the formation of a bimolecular fluorescent complex, which results in a YFP signal. The yellow fluorescence signal appeared in vacuoles and also in autophagosome-like structure (Figure 24C, arrows), further confirming the interaction of both proteins and their localization to vacuoles 
and autophagosomes. As control, fluorescence microscopy was also performed with wild type transformants expressing only SmNBR1-YC or YN-SmATG8, or in combination with the respective empty vector to exclude self-activation (Figure 24D).

SmATG8 is a main component for the formation of the autophagosomes (Voigt and Pöggeler 2013a). Beside SmATG8, the ubiquitin-like autophagy protein SmATG12 was also analyzed to be essential for autophagy in S. macrospora (see 3.1) (Werner et al. 2016). To determine the dependency of SmNBR1 and the core autophagy proteins SmATG12 and SmATG8 during autophagy we observed distribution of fluorescent SmATG8 and SmNBR1 in these deletion mutants. Interestingly, in contrast to the pattern observed in the control strain (complementation strain), the fluorescence signal of the SmNBR1-DsRED fusion protein was detected as aggregates in the cytoplasm but was absent from the vacuoles in the autophagy mutants $\Delta$ Smatg8 and $\Delta$ Smatg12. Furthermore, the dot-like structures were larger and fewer in number than those observed in the control strain. Thus, SmNBR1 was not translocated to the vacuoles, neither in the $\Delta$ Smatg8 nor in the $\Delta \operatorname{Smatg} 12$ knockout mutant, indicating the dependency of the vacuolar localization of SmNBR1 on the core autophagy machinery (Figure 25A). We also analyzed the localization of EGFP-SmATG8 in a $\Delta$ Smnbr1 knockout strain to determine whether the localization of SmATG8 to the vacuole is in turn dependent on SmNBR1 (Figure 25B). However, the EGFP-SmATG8 fusion protein localized to vacuoles and autophagosomes in $\Delta$ Smnbr1, similar to the the complemented strain $\Delta$ Smatg8::egfp-Smatg $8^{\text {ect }}$ (Figure 25B). 

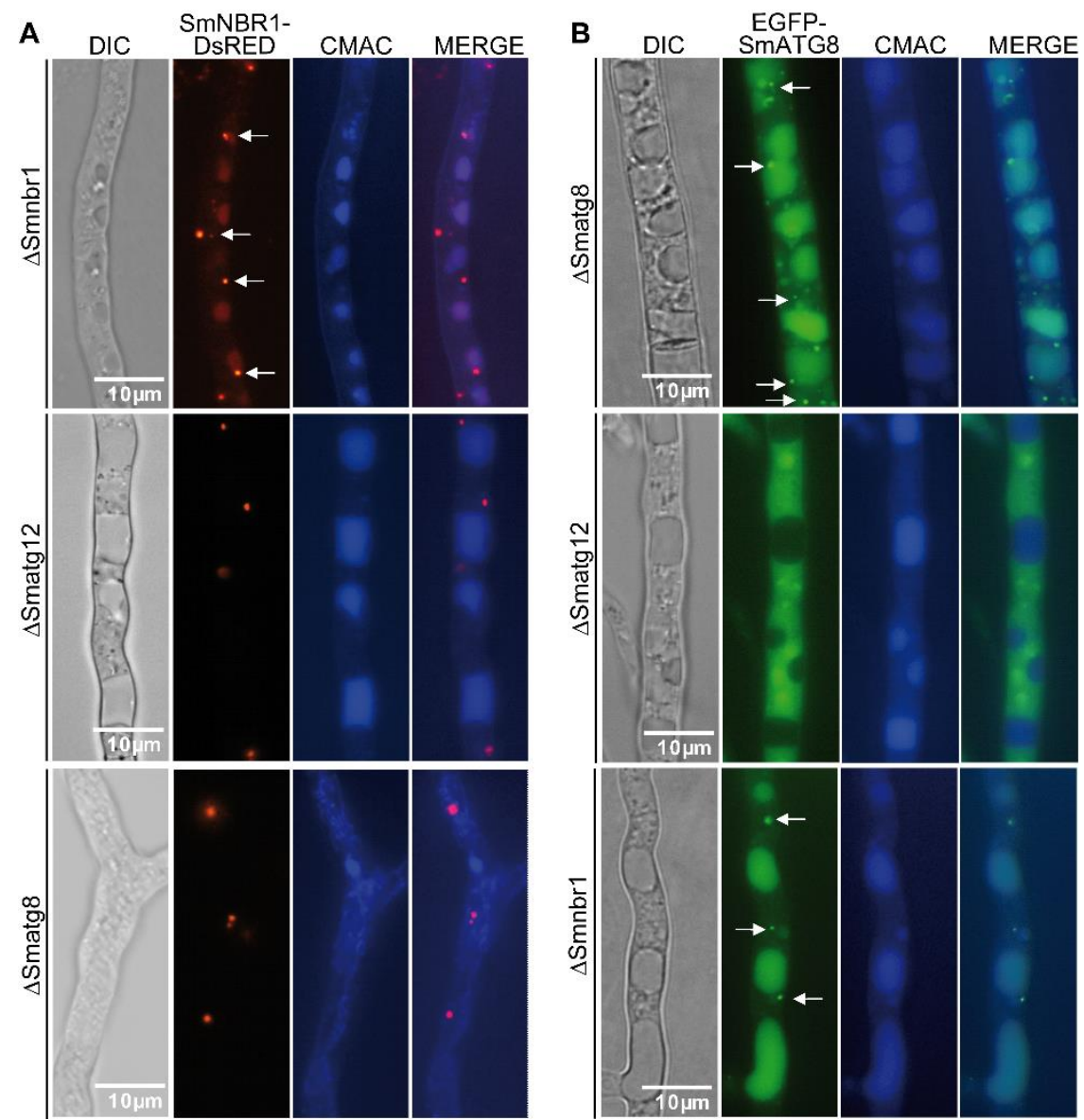

Figure 25: Fluorescence microscopic analysis of S. macrospora SmNBR1 and SmATG8 in different deletion mutants.

(A) The deletion strains $\Delta$ Smnbr1, $\Delta$ Smatg12, $\Delta$ Smatg8 were transformed with $\mathrm{pSmnbr1-Dsred,} \mathrm{expressing} \mathrm{a}$ SmNBR1-DsRED fusion construct. SmNBR1 is localized in the cytoplasm as large aggregates and is excluded from the vacuoles in $\Delta$ Smatg 12 and $\Delta$ Smatg8. Arrows point to autophagosomes. (B) Analysis of EGFP-SmATG8 localization in the $\Delta$ Smnbr1 and $\Delta$ Smatg12 knockout strains and the control strain $\Delta$ Smatg8 each carrying the plasmid pRS-egfp-Smatg8. EGFP-SmATG8 localized in $\Delta$ Smnbrl and $\Delta$ Smatg8 strains to vacuoles and autophagosomes which are indicated by arrows. Vacuoles were stained with CMAC. DIC, differential interference contrast. Scale bars are indicated.

This could be also verified by the quantitative determination of the ratio between the intact autophagy marker protein EGFP-SmATG8 and free EGFP. The EGFP-SmATG8 protein is rapidly degraded in the vacuole whereas the cleaved EGFP moiety is stable in the vacuole (Klionsky et al. 2016). EGFP-SmATG8 is not or slightly degraded in $\Delta$ Smatg4 and in $\Delta$ Smatg12 autophagy mutants. In contrast, vacuolar cleavage of EGFP could be detected in the complementation strain $\Delta$ Smatg8::egfp-Smatg8 $8^{\text {ect }}$ as well as in the $\Delta$ Smnbr1::egfp-Smatg8 ${ }^{\text {ect }}$ strain (Figure 26A). This effect was enhanced by cultivating the strains under autophagyinducing conditions (Supplement 6, BMM+2.5 mM 3-AT). Expression analysis of SmNBR1DsRED in a $\triangle$ Smatg8 or $\triangle$ Smatg12 strain revealed that the SmNBR1-DsRED fusion protein is not degraded (Figure 26B). Hence, vacuolar targeting of SmNBR1 depends on autophagy, but the general autophagy process is not affected in the absence of SmNBR1. 

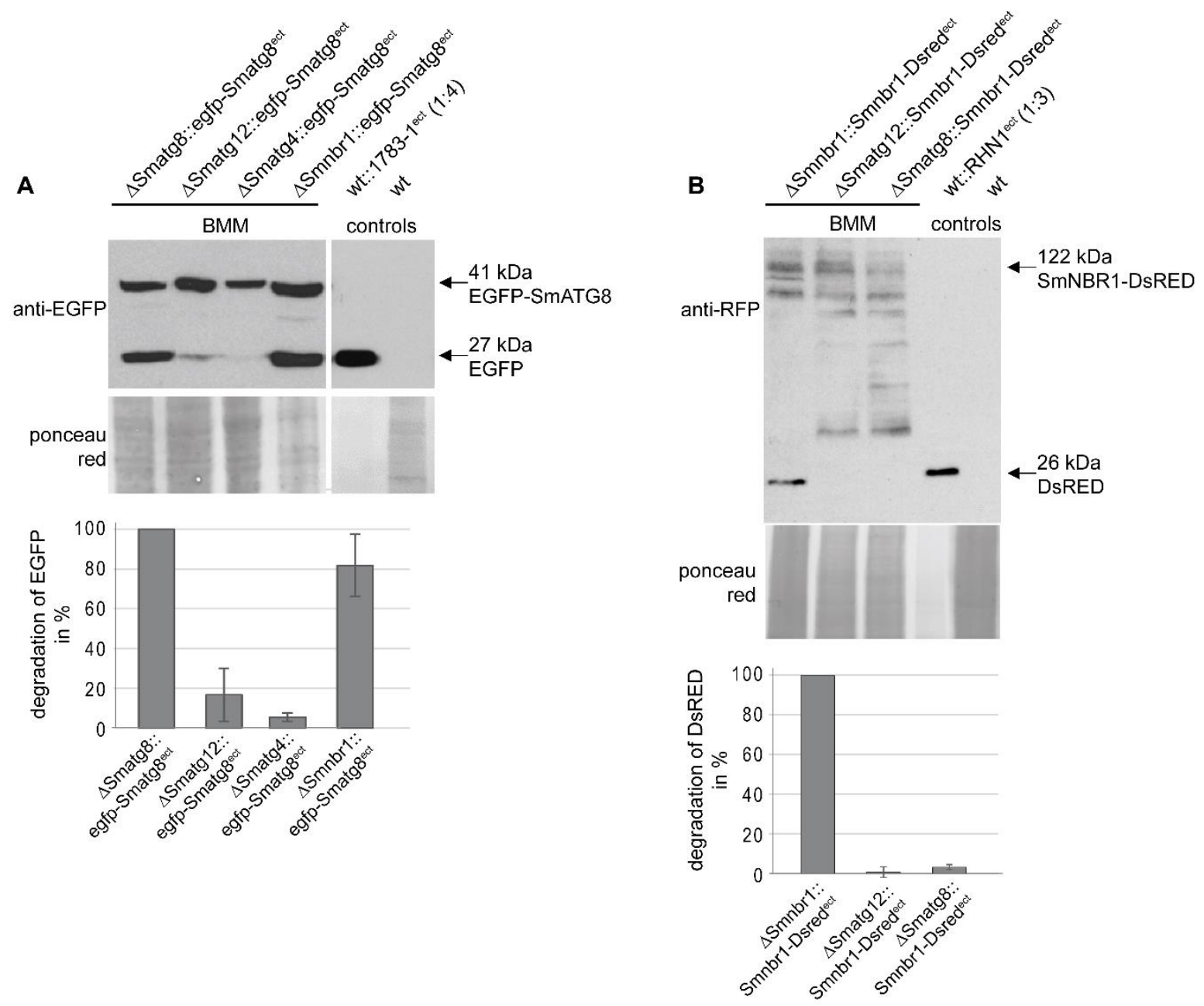

Figure 26: Quantitative Western experiments of the vacuolar degradation of EGFP-SmATG8 or SmNBR1DsRED in different autophagy mutants.

(A) Expression of an EGFP-SmATG8 fusion construct in different autophagy mutants was determined by Western blotting using a monoclonal anti-EGFP antibody. Wt and a strain expressing free EGFP served as controls. The strains were grown for $3 \mathrm{~d}$ in complex media (BMM). The crude protein extracts analyzed were prepared from strains $\quad \Delta$ Smatg8::egfp-Smatg8 $8^{\text {ect }}, \quad \Delta$ Smatg12::egfp-Smatg8 $8^{\text {ect }}, \quad \Delta$ Smatg4::egfp-Smatg8 $8^{\text {ect }}, \quad \Delta$ Smnbr1::egfpSmatg $8^{\text {ect }}$, wt:: $1783-1^{\text {ect }}$ and the untransformed wt strain. Ponceau staining served as loading control to check the protein levels. The program ImageJ was used to quantify the ratio between cleaved EGFP and the EGFP-SmATG8 fusion protein. The cleavage of EGFP in the complemented strain $\Delta$ Smatg8::egfp-Smatg8 ${ }^{\text {ect }}$ was set to $100 \%$. One of three independent experiments is shown. (B) Anti-RFP Western blot of $\Delta$ Smnbr1, $\Delta$ Smatg 12 and $\Delta$ Smatg8 transformed with plasmid pSmnbr1-Dsred. Wt strain and a wt strain expressing a gene coding for the DsRED-tag without any fused protein served as controls. SmNBR1-DsRED shows several bands around $100 \mathrm{kDa}$. The membrane was stained with ponceau red to show the total protein amount. The blot was quantitative analyzed as described in (A). One Western blot is representatively shown. The diagram shows a quantification of the Western blots of the cleaved DsRED and the SmNBR1-DsRED fusion protein. It is an average of three independent experiments. Error bars are indicated. 


\subsubsection{SmNBR1 displays many putative phosphorylation sites}

Since Western blots of SmNBR1 are often associated with many background signals a phosphorylation-site prediction was performed using the software NetPhos3.1 (www.cbs.dtu.dk/services/NetPhos/). The program predicted 113 potential phosphorylation sites comprising 56 serine, 45 threonine and 12 tyrosine residues. Two of the predicted phosphorylation sites are $\mathrm{T}^{843}$ and $\mathrm{Y}^{847}$ of the LIR motif were forecasted to be potential substrates of the common kinases $\mathrm{CK} 2$ and the sarcoma (SRC) kinase, respectively. Additionally, the residues $S^{793 / 804 / 808}$ could be also identified and were potential substrates of the DNA-dependent protein kinase (DNAPK, phosphatidylinositol-3-kinase related kinase) and glycogen synthase kinase 3 (GSK3). To test for phosphorylation of SmNBR1 in vivo we treated total protein extracts of $\Delta$ Smnbr1::Smnbr1-Dsred ${ }^{\text {ect }}$ with phosphatase inhibitor or with lambda phosphatase. The treatment of the protein extracts with phosphatase inhibitor and lambda phosphatase revealed more and less signals as without enzymes, respectively (Figure 27). This strongly suggests that SmNBR1 is indeed a substrate for kinases.
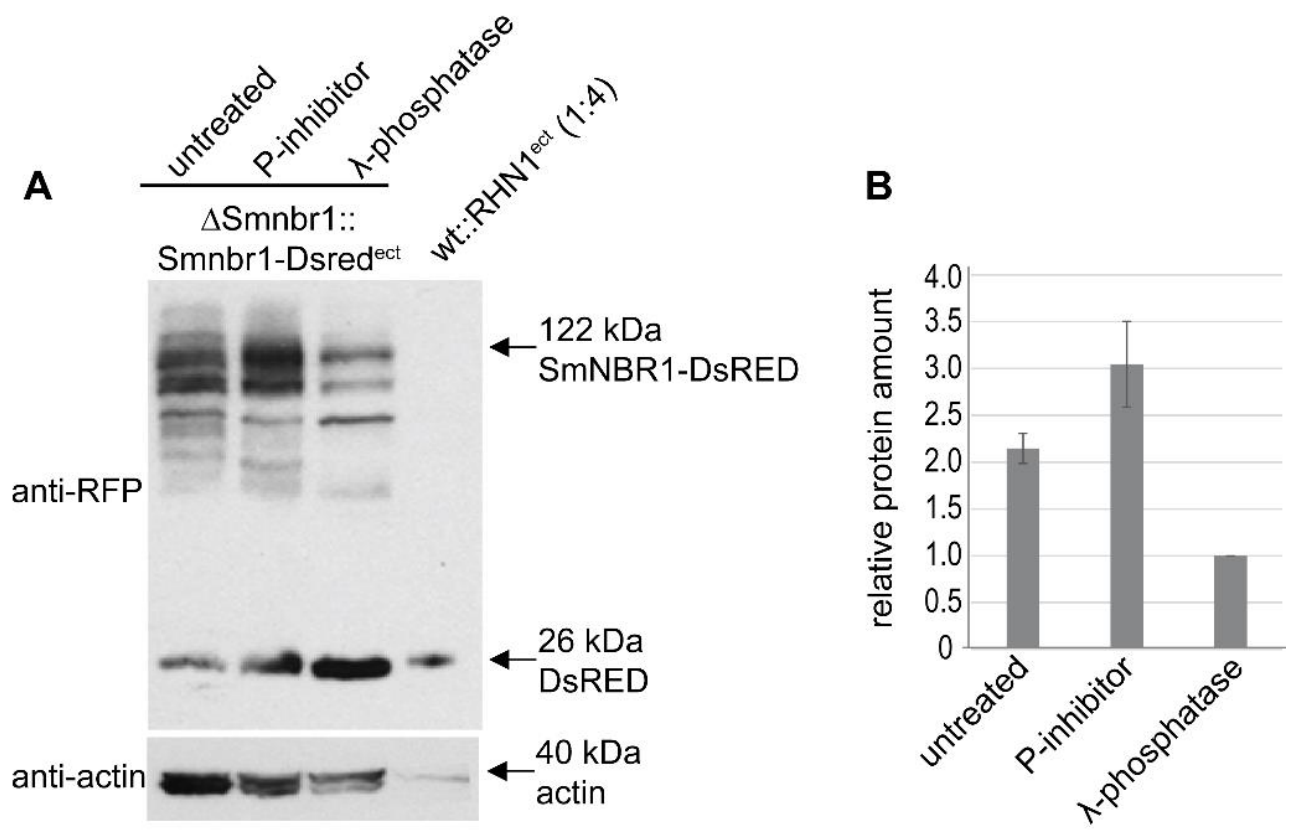

Figure 27: SmNBR1-protein phosphorylation assay.

(A) Western blot showing the treatment of protein extracts of strain $\Delta$ Smnbr1::Smnbr1-Dsred ${ }^{\text {ect }}$ without enzymes (untreated), with phosphatase inhibitor (P-inhibitor) or lambda phosphatase ( $\Lambda$-phosphatase). SmNBR1-DsRED was detected by using anti-RFP. Detection of actin is used to show the protein levels loaded. One of three Westernblot experiments is represented. Protein extract of a wt strain expressing free DsRED was used as control. (B) Quantification of Western blots using the program ImageJ. Data in this diagram are represented for three independent experiments. Error bars are indicated. 


\subsubsection{Overexpression of SmNBR1 has no phenotypical effects, but modifications of SmNBR1 prevent complementation of $\Delta$ Smnbr1 and lead to mislocalization}

For analyzing how SmNBR1 overproduction affects the growth of S. macrospora, a ccg1Smnbrl-egfp overexpression construct was transformed in $\Delta$ Smnbr1. The resulting strain $\Delta$ Smnbr1::ccg1-Smnbr1-egfp ${ }^{\text {ect }}$ behaved like the Smnbrl-egfp complementation strain (compare to Figure 22) and no apparent differences were observed under stress-inducing conditions (Figure 28A) as well as in fluorescence microscopic localization studies (Figure 28B).

The two-hybrid studies suggested that the LIR and the CR motif of SmNBR1 are required for interaction of SmNBR1 with SmATG8. To determine which domains of SmNBR1 are functionally important in vivo, we performed genetic complementation analysis of $\Delta$ Smnbr1 with different Smnbrl variants the same as used for yeast-two hybrid study (3.2.4). All modified Smnbrl versions were tagged with an egfp and expressed under the control of the native promoter and therefore could be localized in vivo by fluorescence microscopy (Figure 28B). Interestingly, all truncated SmNBR1 variants were unable to fully complement the $\Delta$ Smnbr1 phenotype, characterized by less or no production of fruiting bodies and ascospores under normal growth conditions (SWG $27^{\circ} \mathrm{C}, 8 \mathrm{~d}$ ) as well as under stress-inducing conditions (3-AT, $20^{\circ} \mathrm{C}$ ). Also the vegetative growth is inhibited under stress because the strains were unable to cover the plates with mycelium within $8 \mathrm{~d}$ (Figure 28A). These findings correlate with the data of the fluorescence microscopy. Truncated SmNBR1 versions were absent from the vacuole and accumulated in the cytoplasm (Figure 28B).

The double- and single-point LIR motif mutants (Smnbrl-Y ${ }^{847}$ A L ${ }^{850} \mathrm{~A}, \operatorname{Smnbr} 1-\mathrm{T}^{843} \mathrm{~A}$ ) were able to form perithecia and discharge mature ascospores in a reduced number under all conditions. In contrast, the triple-point LIR motif mutant (Smnbr1-T $\left.{ }^{843} \mathrm{~A} \quad \mathrm{Y}^{847} \mathrm{~A} \mathrm{~L}^{850} \mathrm{~A}\right)$ displayed a drastically reduced vegetative growth, number of fruiting bodies and mature ascospores. All LIR-motif mutant proteins were located in the vacuole but they show no or less autophagosomes in the cytoplasm.

Transgenic $\Delta$ Smnbr1 expressing the CR-motif mutant gene Smnbrl $\mathrm{S}^{793} \mathrm{~A} \quad \mathrm{~S}^{804} \mathrm{~A} \quad \mathrm{~S}^{808} \mathrm{~A}$ produced perithecia and ascospores and showed less defects under stress-inducing conditions than LIR-motif mutants. In this strain no apparent differences were observed in the fluorescent localization study in comparison to the complemented strain (Figure 28). 


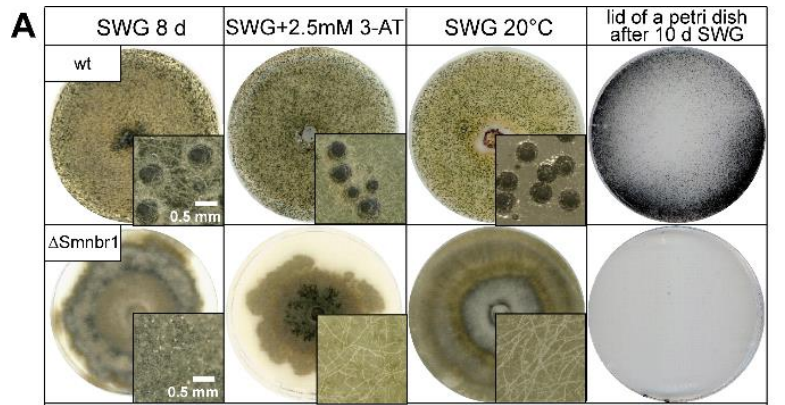

B
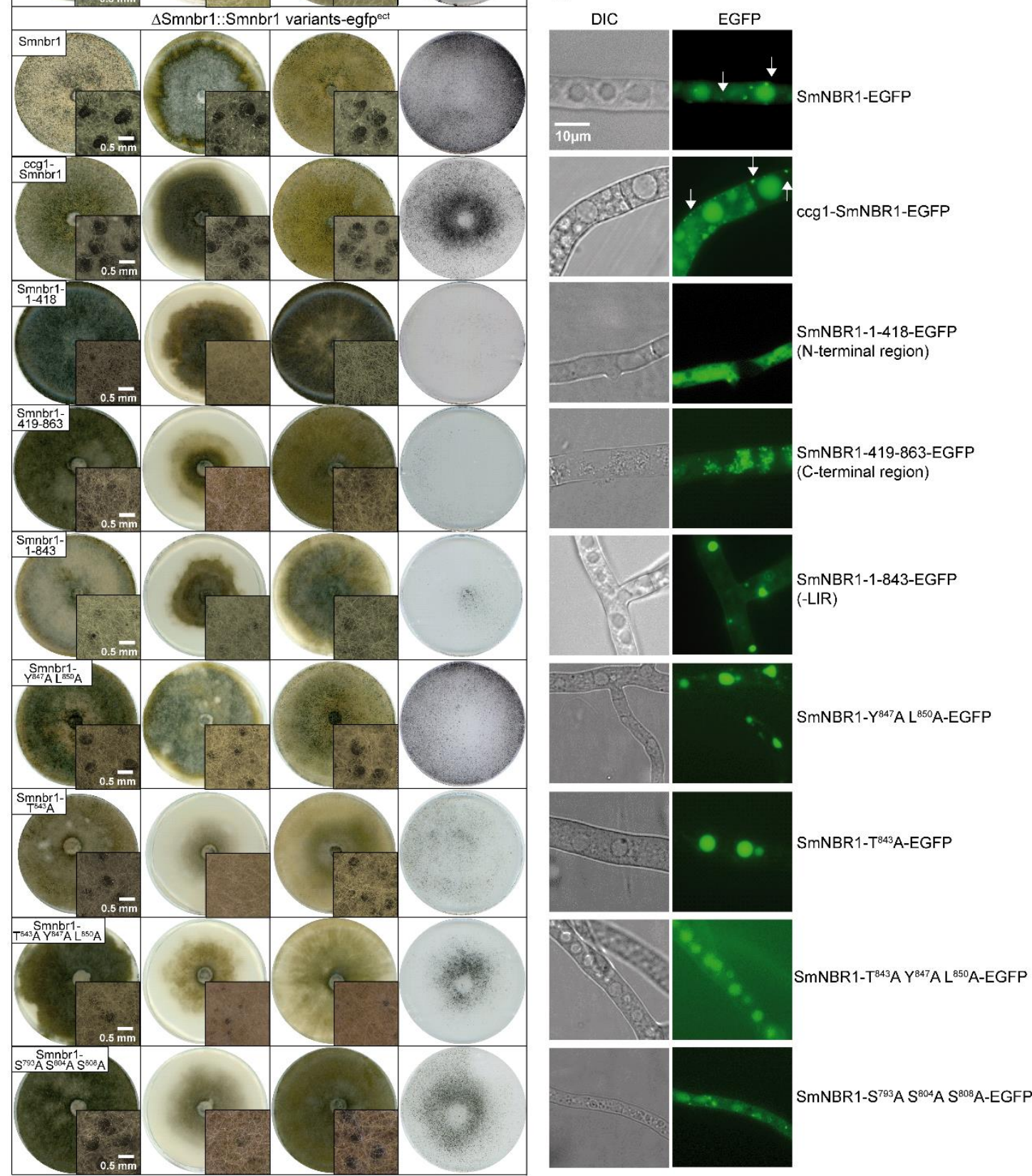

Figure 28: Phenotypical analysis and fluorescence localization studies of $S$. macrospora $\Delta$ Smnbr1 strain transformed with Smnbrl variants under normal, low temperature and autophagy-inducing conditions.

(A) All strains were analyzed under the same conditions as indicated above the panels and compared to the wt control and the $\Delta$ Smnbr1 knockout strain. Images of agar plates as well as the microscopic images were taken after $8 \mathrm{~d}$ of inoculation and after $10 \mathrm{~d}$ the lid of petri dishes with the discharged ascospores were documented. The SmNBR1 protein variants contain an EGFP tag. The analysis was done with three biological replicates in three repetitions. (B) Fluorescence microscopic analysis of S. macrospora $\Delta$ Smnbr1 strains carrying plasmids expressing SmNBR1-EGFP variant fusion constructs. All proteins were detected under the same conditions, cultivated for $24 \mathrm{~h}$ on cellophane on complex media. Scale bars are indicated. Arrows indicate autophagosomes. 


\subsection{SmNBR1-dependent pexophagy in S. macrospora}

In mammals, NBR1 is necessary and sufficient for the turnover of endogenous peroxisomes and acts synergistically with p62 in the pexophagy process (Deosaran et al. 2013; Katarzyna and Suresh 2016). In plants, the NBR1 homolog is involved in aggrephagy and it was speculated that NBR1 may also play a role in pexophagy during heat stress because an over-accumulation of catalases in aggregates of an Atnbrl mutant could be observed (Zhou et al. 2014b; Young and Bartel 2016). However, so far the involvement of plant NBR1 in pexophagy was not experimentally verified.

\subsection{1 $\Delta$ Smnbr1 fails to grow on fatty-acid and $\mathrm{H}_{2} \mathrm{O}_{2}$-containing medium}

In yeasts and filamentous fungi it is known that all steps of $\beta$-oxidation are carried out in peroxisomes, leading to the breakdown of long-chain fatty acids to acetyl-CoA, which is than utilized via the glyoxylate cycle (Hiltunen et al. 2003; Hynes et al. 2008). Peroxisomes contain also various antioxidant enzymes and therefore have an active role in regulating ROS in the cell (Wanders and Waterham 2006).

Using a peroxisomal marker protein we tested selective autophagy in S. macrospora regarding SmNBR1. For this, the wild type, $\Delta$ Smnbr1 and the corresponding complementation strain $\Delta$ Smnbr1::Smnbr1-egfp ${ }^{\text {ect }}$ were grown on SWG medium and the strains were able to utilize glucose as a carbon source. Under this growth condition $\Delta$ Smnbr1 is able to form fruiting bodies and ascospores but in a delayed time and in a drastically reduced number (Figure 29A, Figure 20). In order to analyze the role of SmNBR1 in pexophagy we tested the growth of the $\Delta$ Smnbr 1 mutant on medium containing long-chain fatty acids as carbon source or by inducing oxidative stress by adding $\mathrm{H}_{2} \mathrm{O}_{2}$ to the growth medium. Wild type, $\Delta \mathrm{Smnbr} 1$ and complementation strain were inoculated on SWG medium with reduced $(-75 \%)$ or without glucose together with 0.15 $\%$ oleic acid (C18) as carbon source (Figure 29B) or on SWG medium containing $0.01 \%$ or $0.05 \% \mathrm{H}_{2} \mathrm{O}_{2}$ (Figure 29C). Under these conditions the growth rate of the $\Delta \mathrm{Smnbr} 1$ strain was drastically reduced compared to the wild type and the complemented strain. In addition, no perithecia and consequently no ascospores were formed in the $\Delta$ Smnbr1 strain (Figure 29). These results show that fatty-acid metabolism and oxidative-stress resistance is impaired in the $\Delta$ Smnbr1 strain. Therefore, we tested whether pexophagy is disturbed in $\Delta$ Smnbr1. 
A

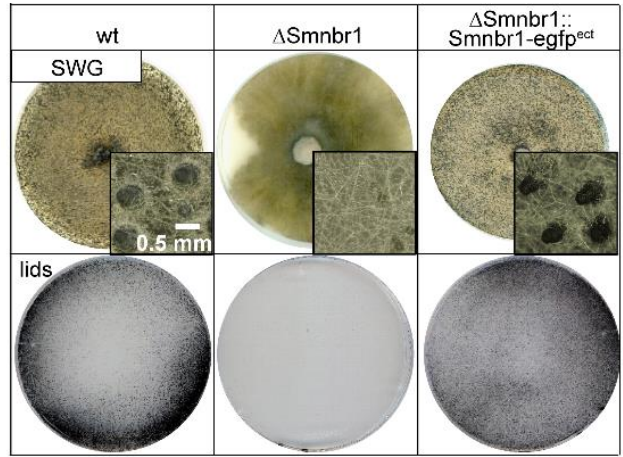

C

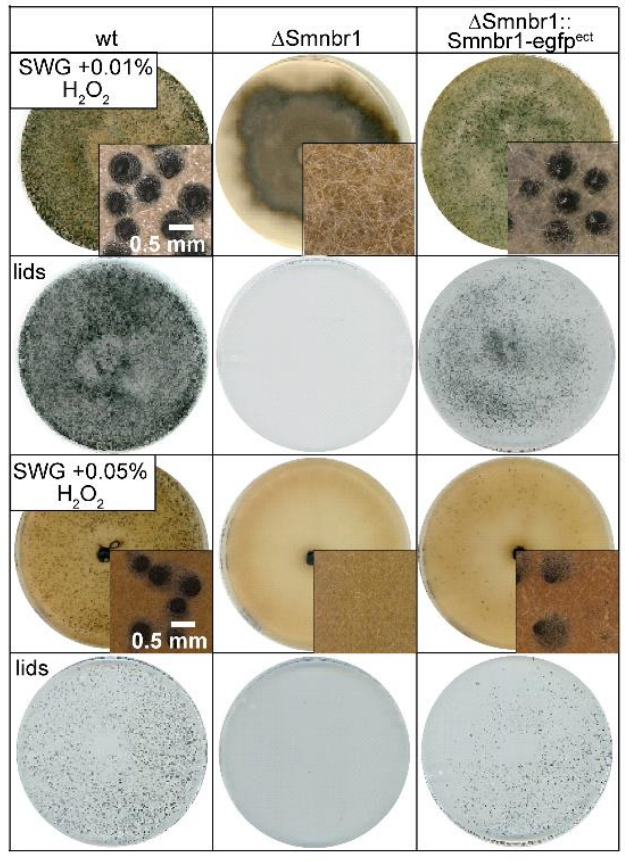

B
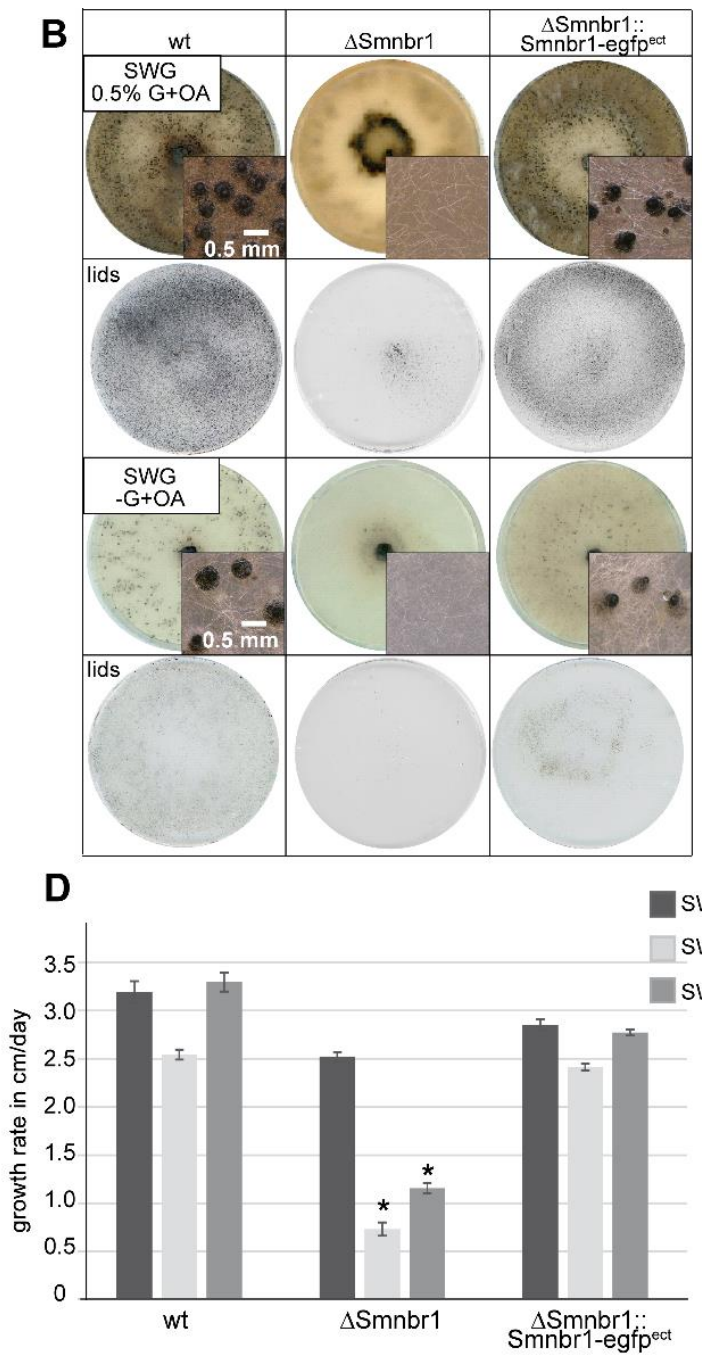

SWG

SWG $0.5 \% \mathrm{G}+\mathrm{OA}$ SWG $+0.01 \% \mathrm{H}_{2} \mathrm{O}_{2}$

Figure 29: Growth analysis of $\mathrm{wt}, \Delta \mathrm{Smnbr} 1$ and complementation strain under peroxisome-proliferation or oxidative-stress conditions.

Phenotypic analysis of wt, $\Delta$ Smnbr1 and $\Delta$ Smnbr1::Smnbr1-egfp ${ }^{\text {ect }}$ grown on SWG medium, oleate medium or under oxidative-stress conditions. Images were taken $8 \mathrm{~d}$ after inoculation. Lids of petri dishes with discharged ascospores were captured after $10 \mathrm{~d}$. Inlets show a magnification of the petri dish. Scale bars are indicated. (A) SWG fructification medium contains glucose as sole carbon source. (B) SWG $0.5 \% \mathrm{G}+\mathrm{OA}$ or without glucose (SWG-G+OA) was supplemented with $0.15 \%$ oleic acid as carbon source. (C) SWG medium was supplemented with $0.01 \%$ or $0.05 \% \mathrm{H}_{2} \mathrm{O}_{2}$. (D) For the same strains, the growth velocity was analyzed using SWG medium (dark grey bars), SWG $0.5 \% \mathrm{G}+\mathrm{OA}$ (light grey bars) or $\mathrm{SWG}$ supplemented with $0.01 \% \mathrm{H}_{2} \mathrm{O}_{2}$ (grey bars). Strains were grown in race tubes for 7 days. Data are means with standard deviations for three biological replicates and three independent experiments. Asterisks show significance analyzed in comparison to wt by students t-test $(\mathrm{p}<0.0000001)$.

\subsubsection{Autophagic degradation of the peroxisomal reporter protein DsRED-SKL arrests in $\triangle$ Smnbr1}

In fungi, peroxisomes are degraded in an autophagy-dependent manner as shown for A. oryzae, P. chrysogenum and S. macrospora (Shoji et al. 2010; Bartoszewska et al. 2011; Voigt and Pöggeler 2013a). Proteins containing a PTS1 or PTS2 sequence can be imported into peroxisomes. The PTS1 sequence is characterized by the amino-acid residues SKL. In S. macrospora wild type, the peroxisomal marker protein DsRED-SKL is localized to punctate 
dots in the cytoplasm and in the vacuole (Elleuche and Pöggeler 2008). However, the vacuolar localization was lost in $\Delta$ Smatg8 and $\Delta$ Smatg4 strains, consistent with the predicted roles of these genes in non-selective pexophagy (Voigt and Pöggeler 2013a). In order to analyze the function of SmNBR1 in pexophagy, localization of peroxisomes was investigated by the use of the DsRED-tagged SKL in wild type and the $\Delta$ Smnbr1 strain. First, we enriched the number of peroxisomes by inoculating the strains on SWG-G+OA medium containing $0.15 \%$ oleic acid (OA) as carbon source. After shifting the strains back to normal growth conditions (SWG) the surplus peroxisomes were degraded in the vacuoles (Figure 30A). The degradation of DsREDSKL in the wild type strain occurs in the vacuoles, which were co-stained with vacuolar dye CMAC blue.

A
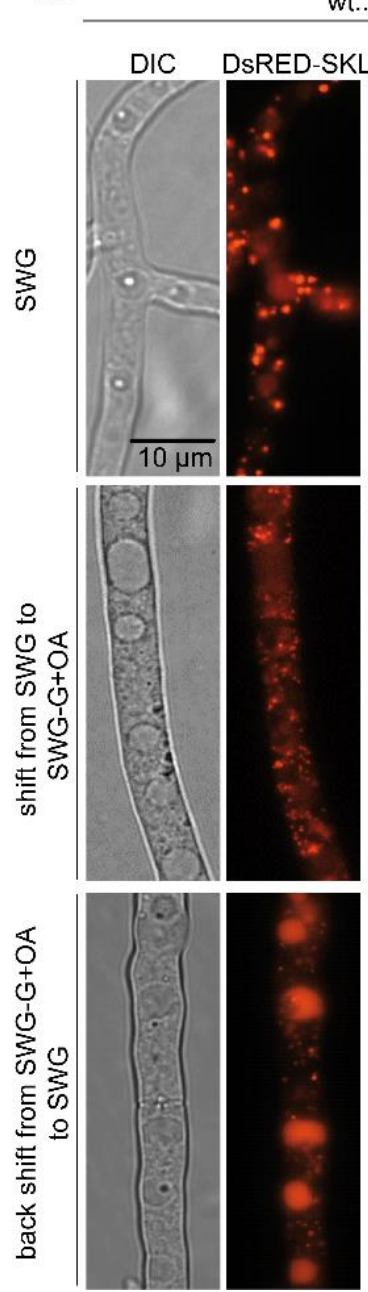

wt::Dsred-SKL ${ }^{\text {ect }}+$ Smnbr1-egfp ${ }^{\text {ect }}$
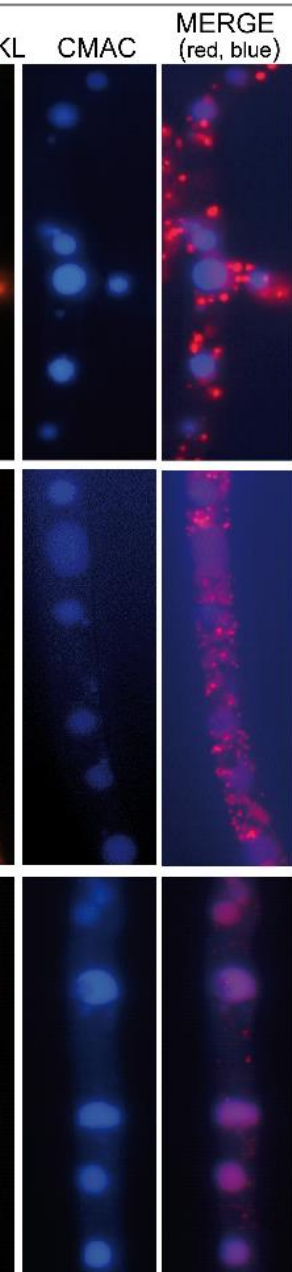

B

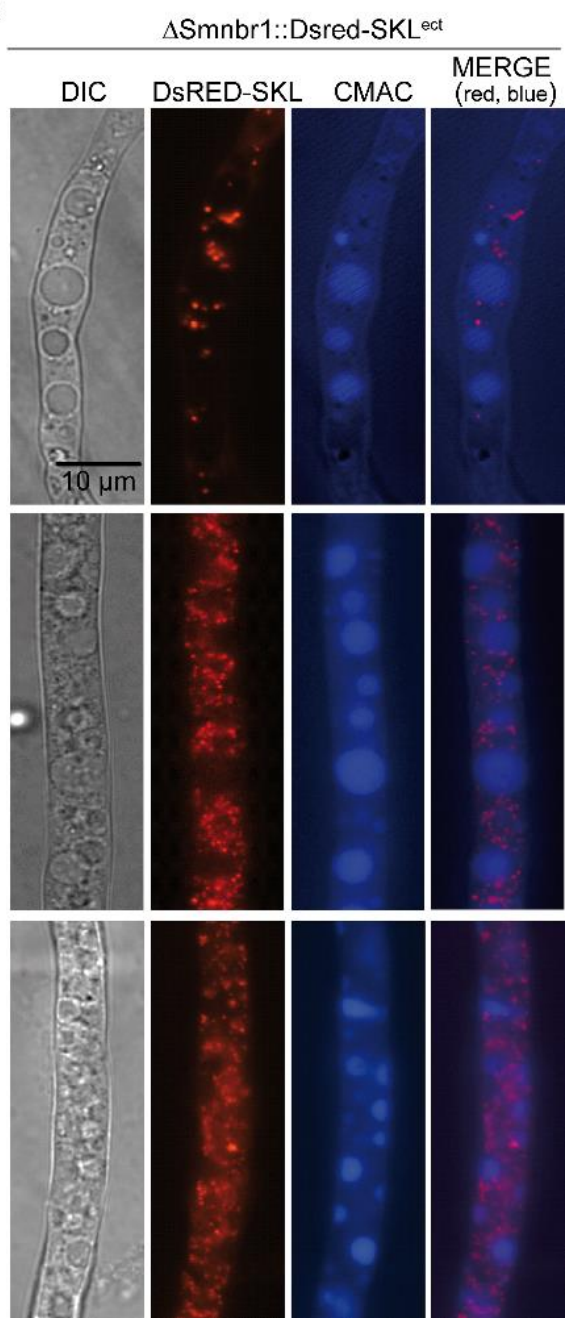

Figure 30: Localization studies of the peroxisomal reporter protein DsRED-SKL in wt and $\triangle$ Smnbr1.

(A) Fluorescence microscopic investigations of wt strain expressing DsRED-SKL and SmNBR1-EGFP for colocalization of both proteins. (B) $\Delta$ Smnbr1 strain expressing the DsRED-SKL fusion protein for visualization of peroxisomes. Strains were grown for $24 \mathrm{~h}$ on SWG, shifted from SWG to SWG-G+OA medium or after shifting back from SWG-G+OA to SWG. The vacuoles were visualized by co-staining the hyphae with the vacuolar dye CMAC. The corresponding DIC and merged images are shown. Scale bars are indicated. DIC, Differential interference contrast. 
DsRED-SKL also co-localizes together with SmNBR1-EGFP in the wild type strain. Both proteins were targeted to the vacuoles for their degradation. In $\triangle$ Smnbr1 DsRED-SKL was only observed in dot-like structures in the cytoplasm but was always absent from vacuoles after shifting the strain from oleate medium to glucose containing medium (Figure 30B).

In conclusion, $\Delta \mathrm{Smnbr} 1$ showed proliferation of peroxisomes on oleic acid medium and the mutant was not able to degrade excess peroxisomes in the vacuole, indicating that pexophagy is impaired in $\Delta$ Smnbr1.

\subsubsection{Peroxisomes change in size, number and morphology in $\Delta$ Smnbr1 and $\Delta$ Smatg8 mutants}

In order to confirm the pexophagy defect of $\Delta$ Smnbrl we analyzed the degradation of the peroxisomal membrane protein SmPEX14.

PEX14 is a peroxisomal membrane protein and an essential component of the peroxisomal import machinery (Kiel et al. 2006). PEX14 of the filamentous fungus $N$. crassa interacted with both targeting signal receptors and was shown to be localized to glyoxysomes (Managadze et al. 2007; Opalinski et al. 2010). To study distribution and morphology of peroxisomes in the $\Delta$ Smnbr1 and a further autophagy mutant, plasmid pSmpex14-egfp expressing SmPEX14EGFP was transformed in wild type, $\Delta$ Smnbr1 and $\Delta$ Smatg8 strains. When a wild type strain expressing SmPEX14-EGFP was grown on glucose and transferred to media containing fatty acids instead of glucose (SWG-G+OA) as sole carbon source, an increased number of peroxisomes was visible after $24 \mathrm{~h}$ and the peroxisomes were degraded in the vacuole after back shifting the strain to normal glucose conditions (Figure 31A). After $24 \mathrm{~h}$ the increased number of peroxisomes was reduced to the nearly optimal level. In contrast, peroxisomes show a different morphology, size and number in $\Delta$ Smnbr1 and the autophagy defective strain $\Delta$ Smatg8. The fluorescence intensity of SmPEX14-EGFP was increased in both strains at all conditions. Additionally, the signals were more punctured and more evenly distributed in the cytoplasm of the mutants than in wild type (Figure 31A). To quantify these observations, the fluorescence signals of SmPEX14-EGFP were analyzed using ImageJ. For this, the fluorescence signals were counted for each strain under different conditions (Figure 31B). The ImageJ quantification correlates with representative fluorescence images. In wild type, an increase of the peroxisomal fluorescence signal under oleate containing medium is followed by a reduction on glucose containing medium. In contrast, $\Delta$ Smnbr1 as well as $\Delta$ Smatg8 displayed an unaltered fluorescence intensity under all conditions. 

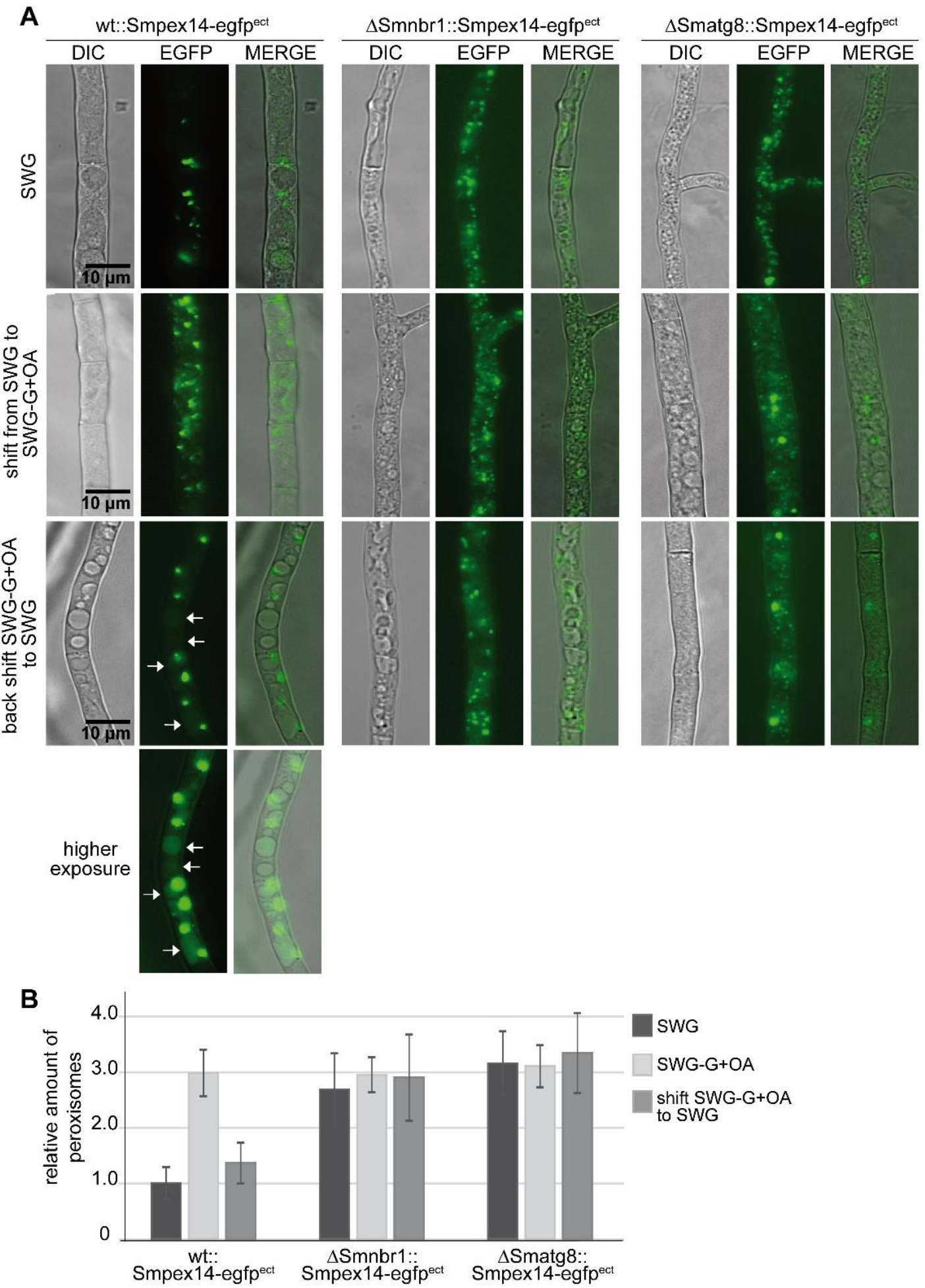

Figure 31: Microscopic and quantitative investigations of proliferation of SmPEX14 EGFP-labeled peroxisomes.

(A) The microscopic images illustrate the fluorescence as well as the corresponding DIC and merged images of SmPEX14-EGFP expressed in wt, $\Delta$ Smnbr 1 and $\Delta$ Smatg8 strains. All strains were analyzed after $24 \mathrm{~h}$ growth on SWG, subsequent growth for $24 \mathrm{~h}$ on SWG-G+OA or $24 \mathrm{~h}$ after shifting the strains from the oleic acid containing media to SWG. For the wt strain, pictures with a higher exposure are shown and filled vacuoles are labeled with a white arrow. These pictures are representative examples for the quantitative analysis shown in (B). Scale bars are indicated. DIC, Differential interference contrast (B) For the quantification of SmPEX14-EGFP fluorescence signals, a defined area in a hyphae (length of $80 \mu \mathrm{m}$ ) was analyzed and counted for EGFP signals with ImageJ. The diagram shows an average of 100 hyphal areas $(n=100)$ for each condition and strain. The standard deviations are indicated. The wt under SWG condition was set to 1 . 
Fluorescence microscopic pictures of wild type strain co-expressing SmPEX14-EGFP and SmNBR1-DsRED revealed an increased number of peroxisome clusters after induction of peroxisome proliferation on fatty-acid containing medium and the back shift to glucose containing medium. Whereas during the starting conditions on SWG medium, more single peroxisomes can be observed. Interestingly, after the medium shift multiple and also single peroxisomes were often decorated with SmNBR1-DsRED (Figure 32). In summary, in S. macrospora the degradation of surplus peroxisomes takes place in a SmNBR1-dependent manner.

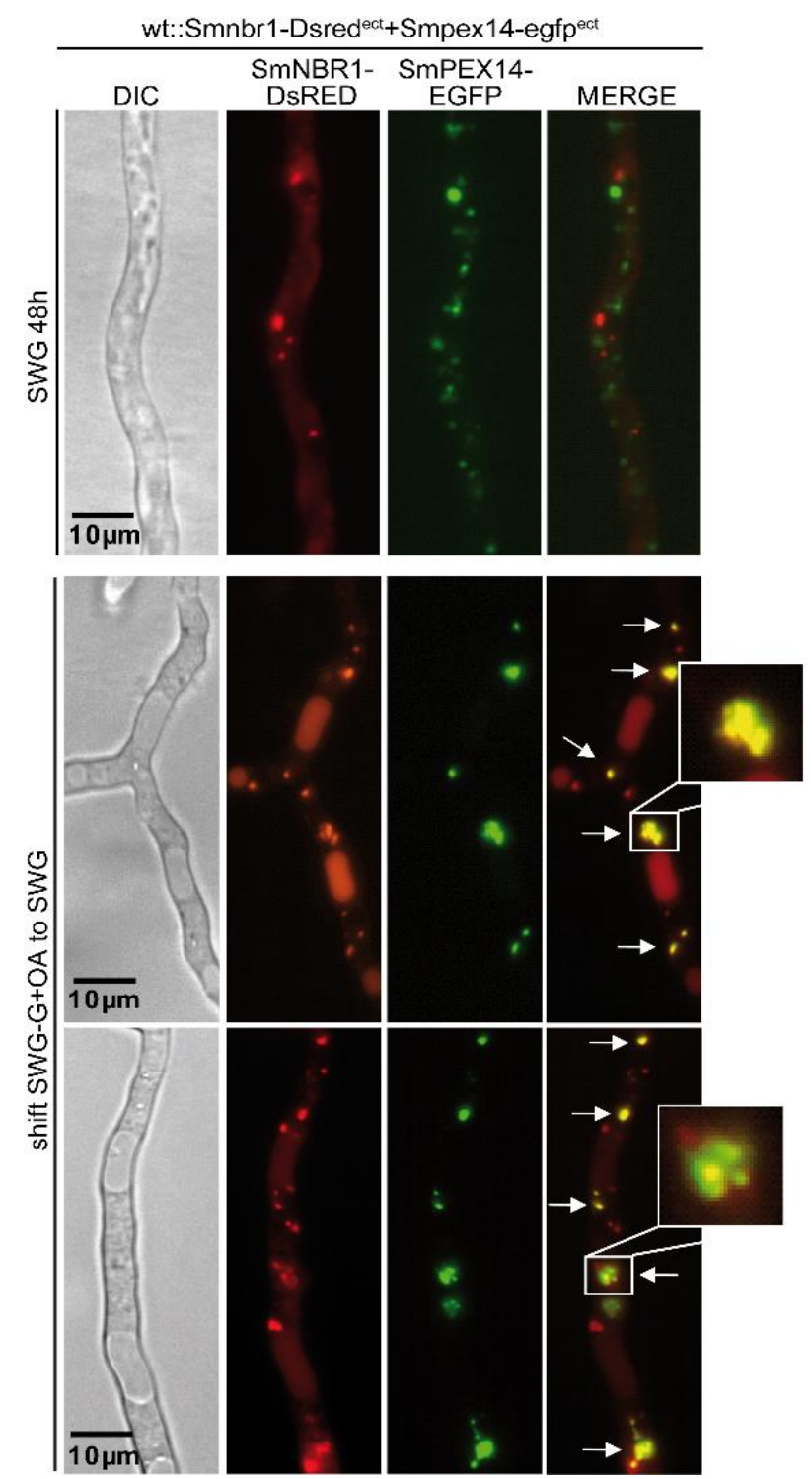

Figure 32: Co-localization studies of SmPEX14-EGFP clusters and SmNBR1-DsRED.

The wt strain expressing SmPEX14-EGFP and SmNBR1-DsRED was analyzed by fluorescence microscopy after $48 \mathrm{~h}$ on SWG or after $24 \mathrm{~h}$ shifting the strains from SWG-G+OA medium w/o glucose instead containing $0.15 \%$ oleic acid (OA) (growth for $24 \mathrm{~h}$ ) to glucose containing medium (SWG). Proliferation of peroxisomes by the formation of cluster structures in response to fatty acids was visualized by peroxisomal membrane labeling with SmPEX14-EGFP. After shifting, the strain single and multiple peroxisomes were decorated with SmNBR1DsRED indicated by arrows. Enlargements show representative co-localization of both proteins. Scale bars are indicated. 


\subsubsection{Functional analysis of Woronin bodies}

Since peroxisomal degradation and turnover is associated with autophagy in S. macrospora, the absence of the autophagy marker protein SmATG8 or the selective cargo receptor SmNBR1 might also affect the function of Woronin bodies. The most abundant protein in this organelle is the matrix protein HEX1. To address whether the autophagy proteins SmNBR1 and SmATG8 are required for Woronin body degradation in S. macrospora, we examined SmHEX1localization in $\Delta$ Smnbr1 and $\Delta$ Smatg8 mutants. For this, a plasmid was constructed which expresses an N-terminal Dsred-tagged version of Smhexl under control of the $\operatorname{trpC}$ promoter of $A$. nidulans. Wild type, $\Delta$ Smatg8 and $\Delta \operatorname{Smnbr} 1 S$. macrospora strains were transformed with the plasmid pDsred-Smhex1. DsRED-SmHEX1 was localized to Woronin-bodies at septal pores and cytoplasm. To investigate the function of Woronin bodies in membrane sealing, hyphae were cut with a scalpel and analyzed 10 min after injury by fluorescence microscopy. In wild type hyphae, the Woronin bodies rapidly sealed the septal pore and thereby prevented the leakage of cytoplasm. After cutting, large puddles of cytoplasm leaked through the hyphal wound which is visible in DIC images (Figure 33). Under the same conditions the localization of DsRED-SmHEX1 in mutant strains $\Delta$ Smnbr1 and $\triangle$ Smatg8 was investigated. As in the wild type, the Woronin bodies were also able to seal the hyphae by plugging the septal pores in the mutant strains.

As control, we analyzed all these strains for the presence of DsRED-SmHEX1. For this, equal amounts of protein crude extracts were analyzed by SDS-PAGE and immunoblotting using an RFP antibody. The S. macrospora DsRED-SmHEX1 protein was detectable in wild type, $\Delta$ Smatg8 and $\Delta$ Smnbr1 strain (Figure 33B). Deletion of Smnbrl and Smatg8 did not seem to disturb localization and functionality of Woronin bodies. 
$\underline{A}$ wt::Dsred-Smhex $1^{\text {ect }}$

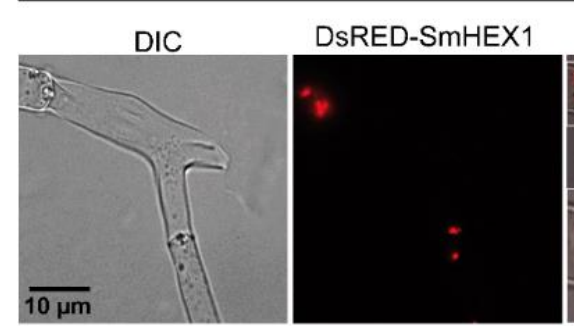

$\Delta$ Smnbr1::Dsred-Smhex $1^{\text {ect }}$ DIC
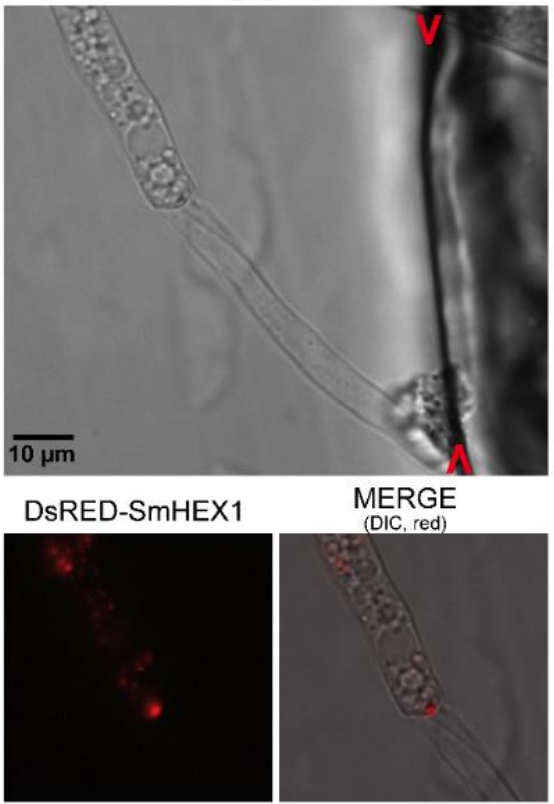

MERGE
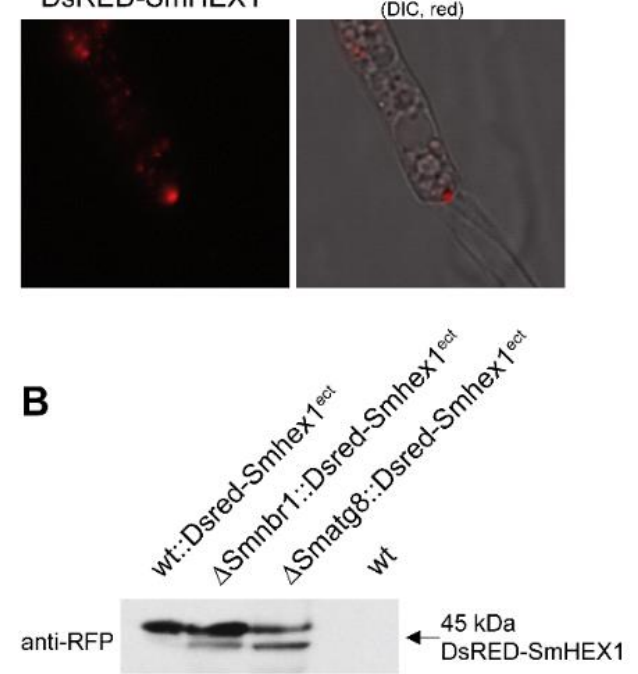

calcufluor white
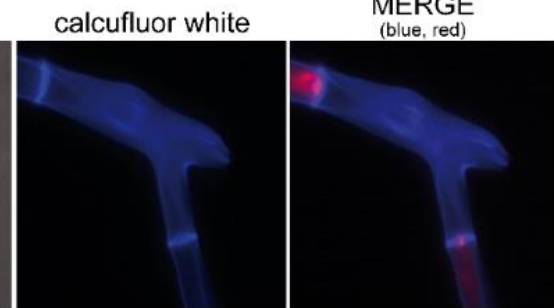

$\Delta$ Smatg8::Dsred-Smhex $1^{\text {ec }}$

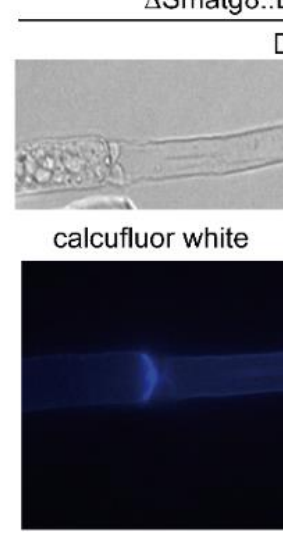

DIC
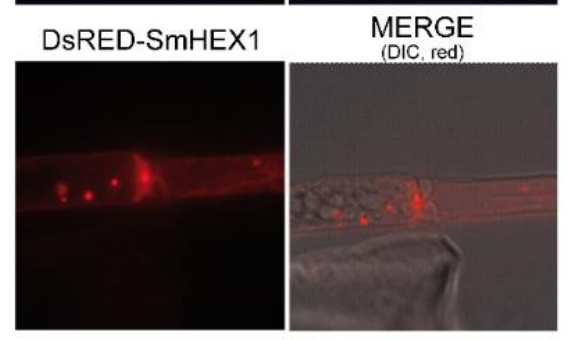

Figure 33: Localization studies of DsRED-SmHEX1 in wt and autophagy mutants.

(A) Fluorescence microscopy of DsRED-tagged Woronin bodies (SmHEX1) in wt, $\Delta$ Smnbr1 and $\Delta$ Smatg8 strains show localization at septal pores and in the cytoplasm. After growing of the strains for $24 \mathrm{~h}$ on covered slides, hyphae were cutted and observed under the microscope after $10 \mathrm{~min}$. Some of the cutting fronts are exemplary labeled with a red open arrows. Septae were stained with calcofluor white. Merged images were assembled from either the fluorescence (red, blue) or the fluorescence and the differential interference contrast (DIC, red) micrographs. Scale bars are indicated. (B) Expression controls of DsRED-SmHEX1 in the same strains by Western-blot analysis of equal amounts of crude extracts using RFP antibody. 


\subsection{SmNBR1-dependent ribophagy in S. macrospora}

In mammals and plants NBR1 acts as an autophagy receptor to target ubiquitinated substrates to the vacuole (Kraft et al. 2010). Since SmNBR1 does not contain an obvious UBA domain we aimed to identify interaction partners of SmNBR1.

\subsubsection{SmNBR1 interacts with ribosomal proteins}

To detect possible interaction partners of SmNBR1 a GFP-Trap analysis coupled to liquid chromatography mass spectrometry (LC/MS) was performed with a strain expressing functional SmNBR1-EGFP as bait protein. The LC/MS data set was analyzed with Proteome Discoverer $^{\mathrm{TM}} 1.4$ (Thermo Scientific) software using the SequestHT search engine (Thermo Scientific). Only high confident proteins, with a threshold of minimum 2 peptides with a false discovery rate less than 0.01 were considered. To identify stringent interactors from the LC/MS raw data set, we included only those proteins that were trapped in two biological replicates and were absent from the control data set obtained from a GFP-Trap of an EGFP-expressing strain. Additionally, a label free quantification (LFQ) was performed with Perseus 1.5.1.0 software and only proteins were chosen which exhibit a t-test significance.

Interestingly, SmNBR1 GFP-Trap analysis revealed two proteins of the small and one large ribosomal subunit as well as the ribosomal associated protein STM1 (suppressor of tom1) within the first 8 best hits of the protein set (Table 7) leading to the assumption that SmNBR1 might be somehow involved in ribosome homeostasis.

Table 7: Results of GFP-Trap and LC/MS analysis using SmNBR1-EGFP as bait protein.

\begin{tabular}{|c|c|c|c|c|}
\hline $\begin{array}{c}\text { No. best } \\
\text { hit }\end{array}$ & SMAC & name & function & $\begin{array}{c}\text { significance } \\
\text { t-test value }\end{array}$ \\
\hline $\mathbf{1}$ & SMAC_07844 & SmNBR1 & selective cargo receptor & $9.25 \mathrm{e}^{+08}$ \\
\hline $\mathbf{2}$ & SMAC_01302 & SmRPS27 & small ribosomal protein 27 & $6.92 \mathrm{e}^{+08}$ \\
\hline $\mathbf{3}$ & SMAC_04779 & $\begin{array}{c}\text { acetyl-CoA- } \\
\text { acetyltransferase }\end{array}$ & fatty acid degradation enzyme & $5.02 \mathrm{e}^{+08}$ \\
\hline $\mathbf{4}$ & SMAC_02849 & $\begin{array}{c}\text { saccharopine } \\
\text { dehydrogenase }\end{array}$ & amino-acid biosynthesis & $2.20 \mathrm{e}^{+08}$ \\
\hline $\mathbf{5}$ & SMAC_00402 & $\begin{array}{c}\text { pseudouridylate } \\
\text { synthase }\end{array}$ & pyrimidine metabolism & $2.13 \mathrm{e}^{+08}$ \\
\hline $\mathbf{6}$ & SMAC_07107 & SmRPL28 & large ribosomal protein 28 & $1.98 \mathrm{e}^{+08}$ \\
\hline $\mathbf{7}$ & SMAC_04909 & SmRPS21 & small ribosomal protein 21 & $1.94 \mathrm{e}^{+08}$ \\
\hline $\mathbf{8}$ & SMAC_05296 & SmSTM1 & $\begin{array}{c}\text { associated with ribosomal proteins } \\
\text { to stabilize these during starvation }\end{array}$ & $1.89 \mathrm{e}^{+08}$ \\
\hline
\end{tabular}


Therefore, ribosomal degradation was monitored in vivo using EGFP-tagged protein SmRPL25 for the large ribosomal subunit and protein SmRPS19 for the small ribosomal subunit. First, the two ribosomal EGFP-fusion proteins were analyzed by fluorescence microscopy in wild type, $\Delta$ Smnbr1 and the autophagy defective $\Delta$ Smatg8 strain.

Under rich conditions (BMM) SmRPL25-EGFP was concentrated in the nucleus (Supplement 7A) of young hyphae in the wild type strain but the EGFP signal accumulated in the cytoplasm and later in the vacuoles in older hyphae indicating that degradation takes place in an autophagy-dependent manner (Figure 34A). In the wild type, vacuolar degradation of SmRPL25-EGFP occured faster under autophagy-inducing conditions (BMM + $2.5 \mathrm{mM} 3-\mathrm{AT})$. In contrast, SmRPL25-EGFP was more stable over a time period in $\Delta$ Smnbr1 and the EGFP signal appeared to be concentrated in the nucleus for a longer period under rich as well as under autophagy-inducing conditions. As expected, SmRPL25-EGFP was very stable and no vacuolar degradation was visible in the autophagy defective mutant $\Delta$ Smatg8. Thus leading to the assumption that the large ribosomal subunit is degraded in a SmATG8 as well as SmNBR1 dependent manner (Figure 34C). 

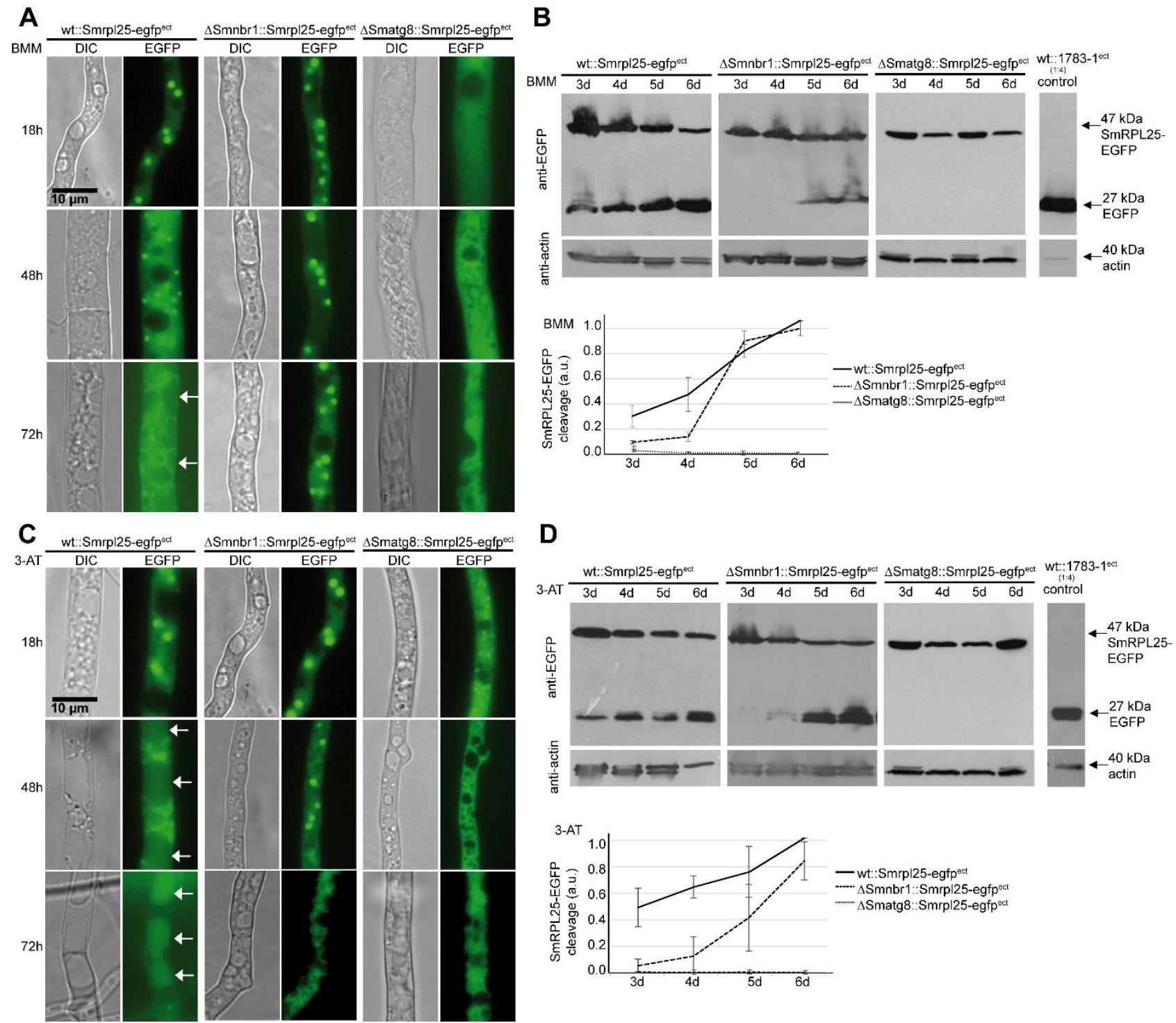

Figure 34: Degradation of SmRPL25-EGFP under normal and starvation conditions.

Wt, $\Delta$ Smnbr1 and $\Delta$ Smatg8 strains expressing SmRPL25-EGFP under the native promoter were grown under normal conditions (BMM) or autophagy-inducing conditions (BMM $+2.5 \mathrm{mM} 3-\mathrm{AT})$. (A) Fluorescence microscopy of SmRPL25-EGFP in the wt and mutant strains after 18, 48 and $72 \mathrm{~h}$ of growth on rich medium. Arrows label filled vacuoles. Scale bar is indicated. (B) Quantitative Western-blot analysis of SmRPL25-EGFP in the same strains as in (A) grown in BMM rich medium for three to six days. Vacuolar EGFP cleavage was analyzed by Western blotting with an EGFP-antibody. ImageJ software was used to quantify the ratio between cleaved EGFP and the full-length protein. The last time point $(6 \mathrm{~d})$ of wt was set to 1 . In the diagram the average of three independent experiments is shown and the standard deviations are displayed. As protein loading control the actin protein was detected using an actin-antibody. A wt strain expressing free EGFP was used as control for EGFP. (C) Strains were grown under autophagy-inducing conditions on BMM supplemented with $2.5 \mathrm{mM} 3$-AT and analyzed by fluorescence microscopy at the indicated time points. Arrows label filled vacuoles. Scale bar is indicated. (D) Quantitative Western-blot analysis of the ribosomal protein SmRPL25-EGFP in the same way as described in (B) under autophagy inducing conditions (BMM +2.5 mM 3-AT). DIC, Differential interference contrast. a.u., arbitrary unit. Scale bars are indicated.

The localization study of SmRPS19-EGFP by fluorescence microscopy revealed the same as for SmRPL25-EGFP, degradation took place in the wild type whereas a stabilization of SmRPS19-EGFP is visible in $\Delta$ Smnbr1 and the autophagy defective mutant $\Delta$ Smatg8 (Figure $35 \mathrm{~A}+\mathrm{C})$. The localization of SmRPS19-EGFP in wild type strain to the nucleus is shown by 
DAPI staining after $24 \mathrm{~h}$ growth. Additionally, after $48 \mathrm{~h}$ growth SmRPS19-EGFP and SmNBR1-DsRED were co-localized in the vacuole shown in the wild type strain expressing both proteins (Supplement 7B,C).

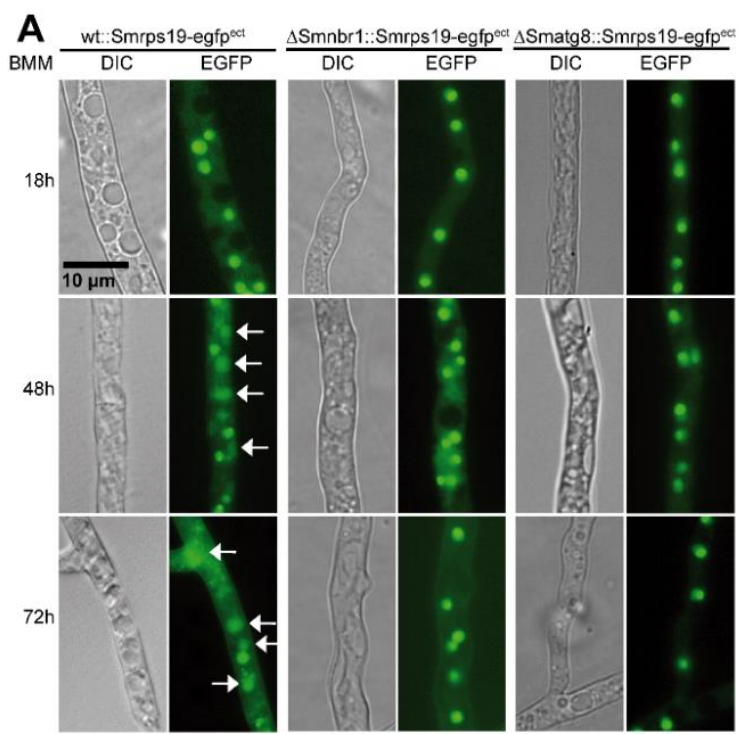

\section{B}

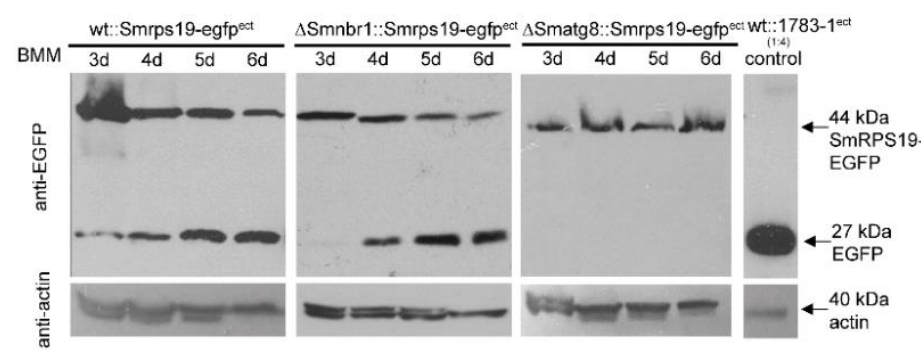

C wt.:Smrps19-egfpet $^{e s} \Delta$ Smnbr1::Smrps19-egfpect $\Delta$ Smatg8::Smrps19-egfpect
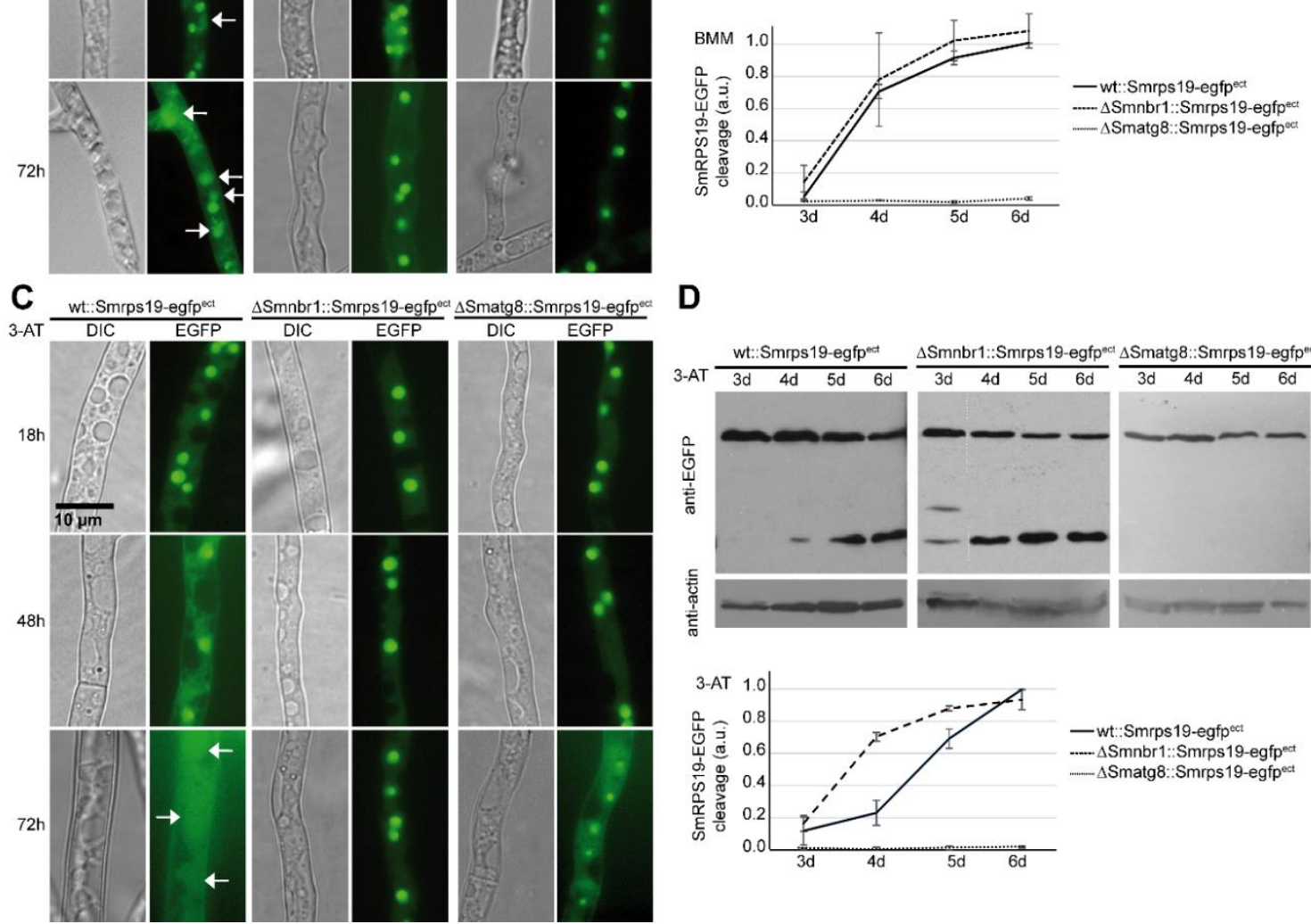

D
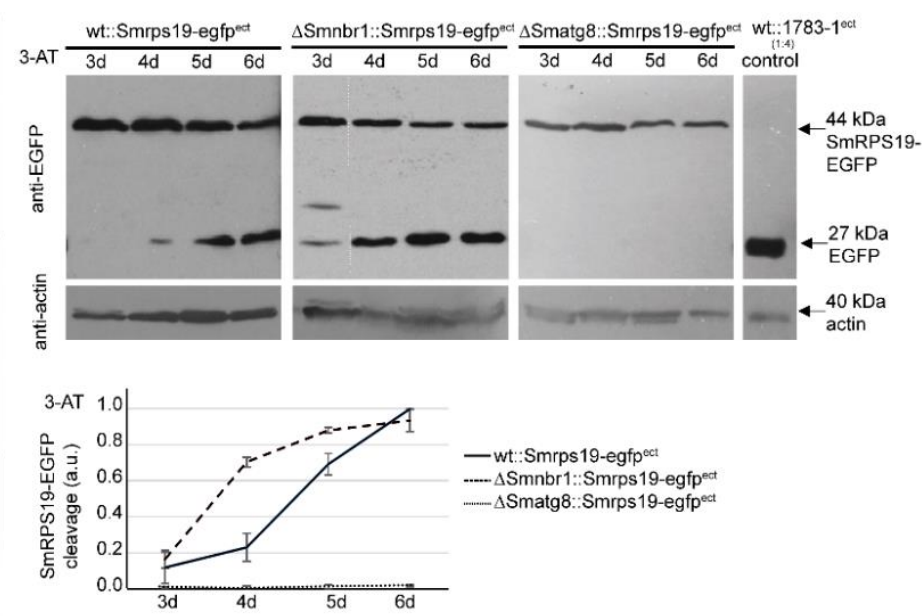

Figure 35: Degradation of SmRPS19-EGFP under normal and starvation conditions.

Wt, $\Delta$ Smnbr1 and $\Delta$ Smatg8 strains expressing SmRPS19-EGFP under the native promoter were grown under normal conditions (BMM) or autophagy-inducing (BMM $+2.5 \mathrm{mM} 3-\mathrm{AT}$ ) conditions. (A) Fluorescence microscopy of SmRPS19-EGFP in the wt and mutant strains after 18, 48 and 72h of growth on rich medium. Arrows label filled vacuoles. Scale bar is indicated. (B) Quantitative Western-blot analysis of SmRPS19-EGFP in the same strains on BMM rich medium for three to six days. Vacuolar EGFP cleavage was analyzed by Western blotting with an EGFP-antibody. ImageJ software was used to quantify the ratio between cleaved EGFP and full length protein. The last time point $(6 \mathrm{~d})$ of wt was set to 1 . In the diagram the average of three independent experiments is shown and the standard deviation is displayed. As protein loading control the actin protein was detected using an actin-antibody. A wt strain expressing free EGFP was used as control for EGFP. (C) Strains were grown under autophagy-inducing conditions on BMM supplemented with $2.5 \mathrm{mM} 3$-AT and analyzed by fluorescence microscopy at the indicated time points. Arrows label filled vacuoles. Scale bar is indicated. (D) Quantitative Western-blot analysis of the ribosomal protein SmRP19-EGFP in the same way as described in (B) under autophagy inducing conditions (BMM +2.5 mM 3-AT). DIC, Differential interference contrast. a.u., arbitrary unit. Scale bars are indicated. 
As control, the localization of free EGFP was analyzed in wild type and $\Delta$ Smnbrl and showed an evenly distributed signal in the cytoplasm. Over the time, this signal was localized to the vacuole (Figure 36).

For the specific relocalization of the ribosomal proteins into the vacuole a functional autophagy machinery is required and it seems that selective autophagy components like SmNBR1 are necessary.

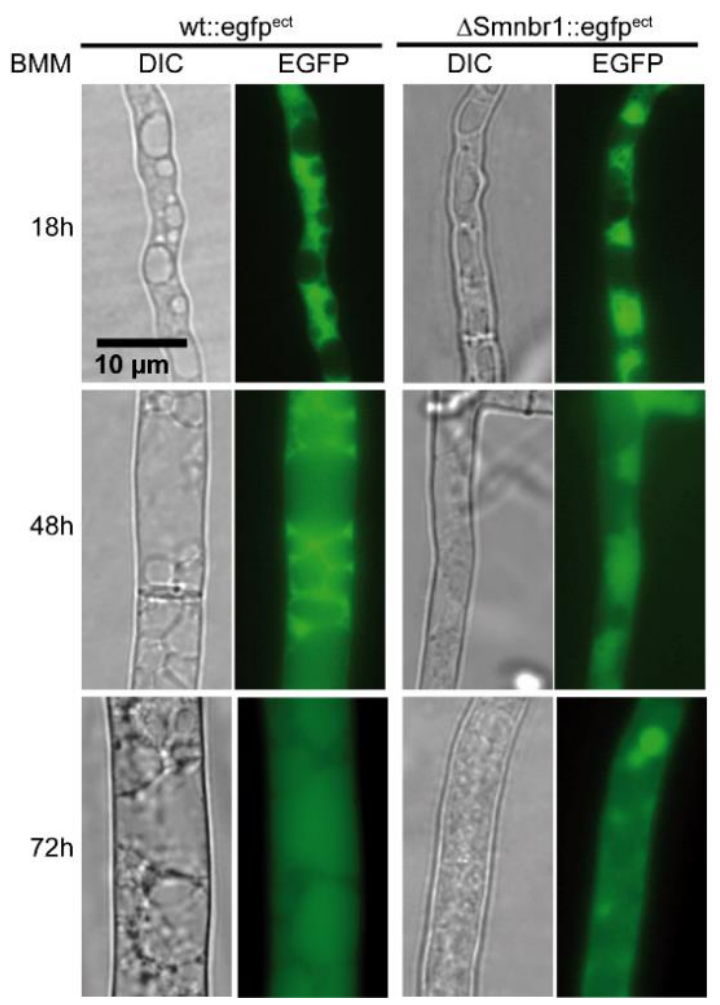

Figure 36: EGFP fluorescence localization in wt and $\Delta$ Smnbr1.

$\mathrm{Wt}$ and $\Delta$ Smnbrl strains expressing EGFP were analyzed by fluorescence microscopy after growth for 18,48 and $72 \mathrm{~h}$ on rich medium. Wt strain was transformed with plasmid p1783-1 and $\Delta$ Smnbr1 with plasmid pDS23. DIC, Differential interference contrast. Scale bars are indicated.

To verify these fluorescence microscopic observations the transport of EGFP-tagged ribosomal proteins from the cytoplasm to the vacuole, was assayed by Western blot analyses of the degradation of the tagged protein and vacuolar accumulation of free EGFP. During vacuolar degradation the EGFP moiety is highly stable and can be monitored as free EGFP by Western blotting of total cell extracts (Kraft et al. 2008). Deletion of Smnbrl led to delayed cleavage of EGFP from the reporter protein SmRPL25-EGFP under normal growth conditions and is even much more delayed after induction of autophagy compared to wild type (Figure 34B,D).

In the autophagy mutant $\Delta$ Smatg8 autophagic degradation of ribosomes is arrested. Therefore, a stable EGFP signal of the fusion protein and no free EGFP signal was detected in the Western blot. This indicated that the cargo receptor SmNBR1 is required for optimal degradation rates of the large ribosomal subunit.

In contrast to this, the Western blot analyses of SmRPS19-EGFP revealed no significant degradation differences in the wild type and $\Delta$ Smnbr1 strain under rich and starvation 
conditions (Figure 35B,D). Similar to SmRPL25, SmRPS19-EGFP was stabilized in the autophagy-defective mutant $\Delta$ Smatg8, which indicated that also the small ribosomal subunit was degraded in an autophagy dependent manner. However, it seems that SmRPS19 was not a substrate for the selective cargo receptor SmNBR1.

To control the function of the bulk autophagy mechanism, the degradation of a cytosolic control protein EGFP-SmATG8 was also analyzed by Westen blot experiments. For this the respective complementation strain $\Delta$ Smatg8::egfp-Smatg8 ${ }^{\text {ect }}$ and the $\Delta$ Smnbr1::egfp-Smatg $8^{\text {ect }}$ strain were used. Both strains had nearly the same degradation ratio of EGFP-SmATG8 (Figure 37).

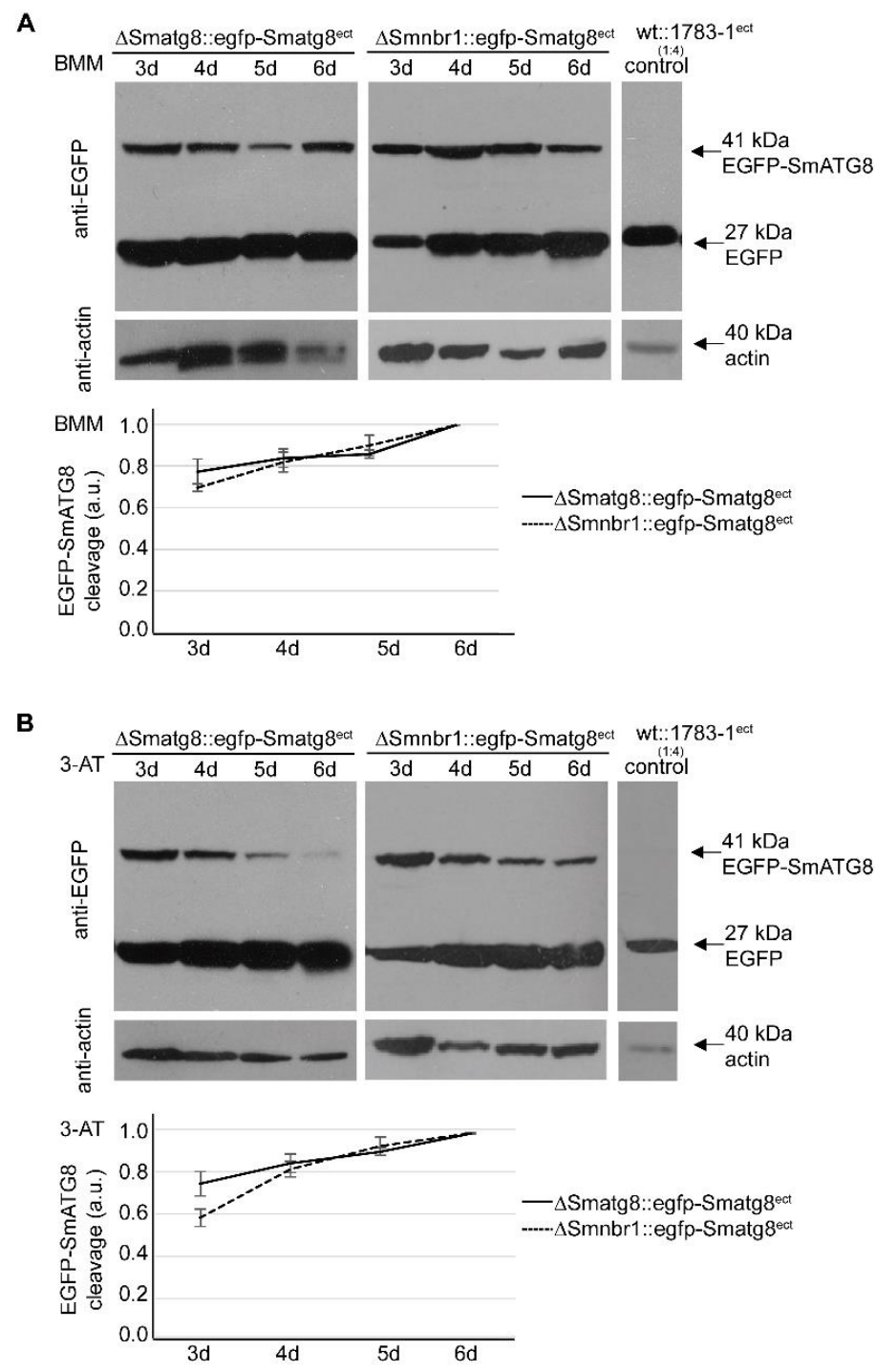

Figure 37: Quantitative Western-blot analysis of the autophagosomal reporter protein EGFP-SmATG8. (A) $\Delta$ Smatg8 and $\Delta$ Smnbr1 strains expressing EGFP-SmATG8 were analyzed under normal growth conditions (BMM) using anti-EGFP antibody. Total protein samples were taken at the indicated time points. One of three representive blots is shown. Anti-actin was used for an equal protein loading. A wt strain expressing free EGFP was used as control for EGFP. The diagram shows the average of three independent experiments with standard deviations. (B) $\Delta$ Smatg8 and $\Delta$ Smnbr1 strains expressing the same control protein were analyzed under autophagy inducing conditions (BMM + 2.5 mM 3-AT) in the same way as described in (A). a.u., arbitrary unit. 
The localization of EGFP-SmATG8 was also analyzed by fluorescence microscopy in the same strains. SmATG8 localized in autophagosomes and in vacuoles. Thus, the protein behaved functional in both strains (Figure 25B) and general autophagy appeared to be not affected in the $\Delta$ Smnbr1 strain.

To analyze whether the cargo receptor SmNBR1 can interact with SmRPL25 or SmRPS19 in vivo, we performed immunoprecipitation (IP) experiments in wild type strains expressing DsRED-tagged SmNBR1 together with either SmRPL25 or SmRPS19 tagged with EGFP. SmRPL25-EGFP could be immunoprecipitated together with SmNBR1-DsRED supporting the hyphothesis for a direct or indirect interaction of both proteins (Figure 38A). In contrast, the SmRPS19-EGFP could not be purified together with SmNBR1-DsRED in the IP experiment (Figure 38B).
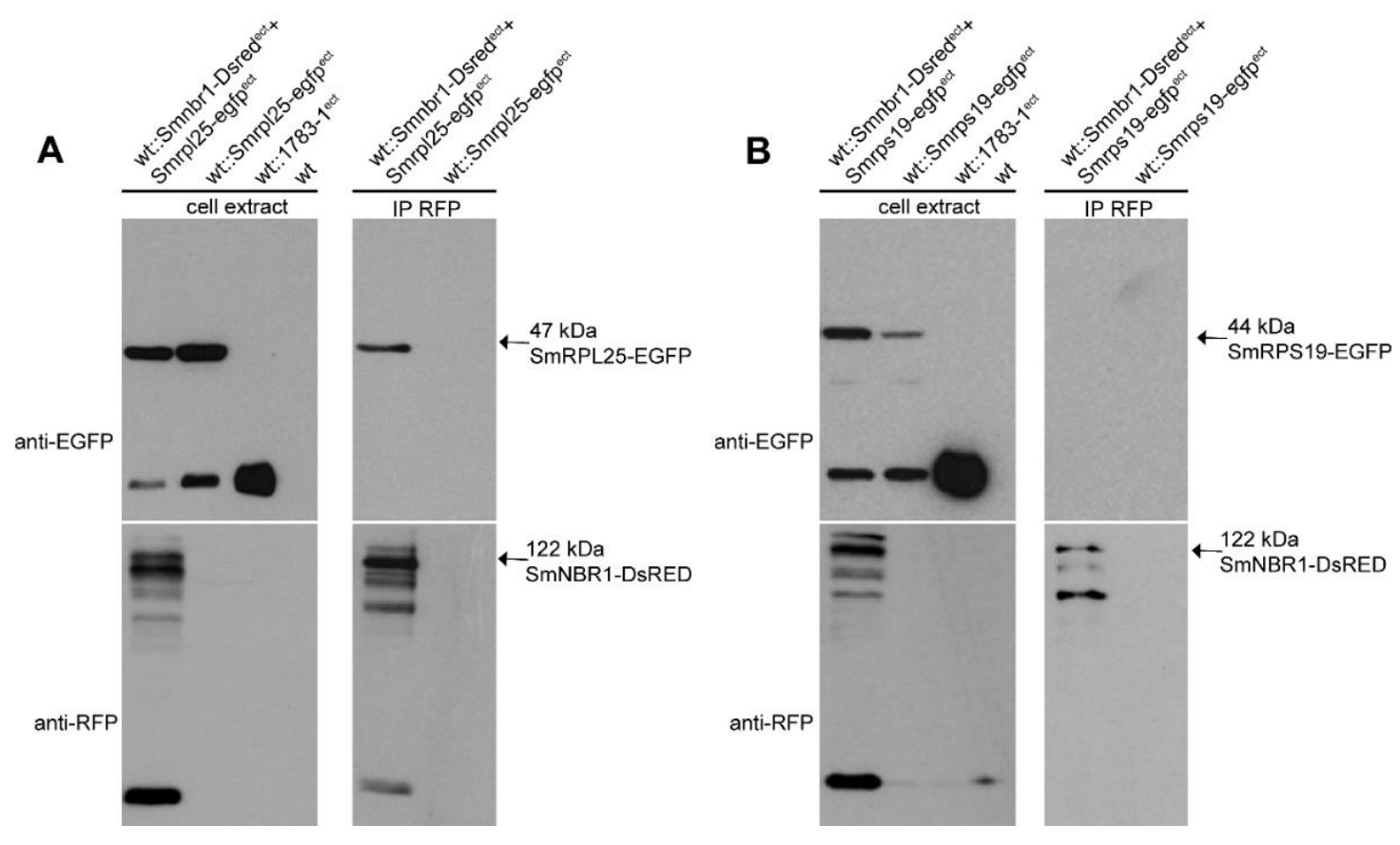

Figure 38: Immuniprecipitation of SmNBR1 together with ribosomal proteins.

(A) Immunoprecipitation using a wt strain expressing SmNBR1-DsRED and SmRPL25-EGFP. After purification of SmNBR1-DsRED the protein SmRPL25-EGFP could be detected using anti-EGFP in the Western-blot analysis. (B) IP as described in (A) but using a wt strain expressing SmNBR1-DsRED and SmRPS19-EGFP. SmRPS19EGFP does not interact with SmNBR1. Untransformed wt strain, a wt strain expressing free EGFP and the respective wt strains expressing SmRPL25-EGFP and SmRPS19-EGFP, respectively, served as controls. The strains were grown for $3 \mathrm{~d}$ in rich medium (BMM). The crude protein extracts were prepared and analyzed using anti-GFP and anti-RFP antibodies. 
This result together with the quantitative Western-blot experiments suggested that SmRPS19 and maybe 40S ribosomal subunits in general are no direct cargos of SmNBR1.

Additionally, SmRPL25 and SmRPS19 were tested in a yeast-two hybrid assay for direct interaction with the cargo receptor SmNBR1. Neither SmRPL25 nor SmRPS19 showed an interaction with SmNBR1 leading to the assumption that both proteins are no direct substrates of the cargo receptor (Supplement 8). 


\section{Discussion}

\subsection{SmATG12, a core autophagy protein}

In contrast to ATG8 homologs, the function of the second UBL autophagy protein ATG12 has received much less attention in filamentous fungi. We previously have shown that the E1-like enzyme SmATG7, which presumably activates SmATG8 and SmATG12, is essential for viability in S. macrospora, whereas the UBL protein SmATG8 and its protease SmATG4 are involved in vegetative growth and sexual development (Nolting et al. 2009; Voigt et al. 2014). We therefore investigated the role of the second UBL protein SmATG12 in sexual development and perithecia formation in this filamentous ascomycete.

\subsubsection{The conserved autophagy-associated protein SmATG12 is unable to rescue the $S$. cerevisiae atg12 $\Delta$ mutant}

Sequencing of the Smatg12 cDNA verified the presence of a C-terminal glycine in the reduced amino-acid sequence of SmATG12 that is essential for the formation of an isopeptide bond with ATG5 (Mizushima et al. 1998) and was only reported to be absent in the Plasmodium falciparum ATG12 ortholog (Kitamura et al. 2012). In addition, the carboxy-terminal region (aa 72-159) of SmATG12 revealed a high degree of sequence identity when compared with ATG12 homologs from S. cerevisiae, plants and animals (Figure 8). This region of SmATG12 was predicted to form an ATG12 ubiquitin-like domain (APG12_C, cd00196) by both the NCBI Conserved Domain Search tool (Marchler-Bauer et al. 2015) and InterProScan (PF04110) (Jones et al. 2014). Within this domain, several amino acids and a turn-loop-alpha helix 2 segment, previously shown in S. cerevisiae and H. sapiens ATG12 to be important for noncovalent interactions with ATG5, were found to be conserved in SmATG12 (Noda et al. 2013; Otomo et al. 2013). In S. macrospora, SMAC_08343 encodes a 366 aa ATG5 ortholog that is larger than human or yeast ATG5 due to insertions, but it contains a conserved lysine residue (K218) that may form a conjugate with the C-terminal SmATG12 glycine residue (Supplement 1). Yeast-two hybrid analysis revealed that SmATG12 can interact with SmATG7, which was previously shown to display characteristic features of E1-like enzymes (Nolting et al. 2009) (Figure 9A). In yeast and mammals, ATG7 activates ATG12 via C-terminal adenylation, and subsequently a conserved cysteine residue is covalently linked via a thioester bond to the $\mathrm{C}$ terminal glycine residue. A similar mechanism may therefore operate in S. macrospora. 
After conjugation to ATG5, the ATG12 ATG5 conjugate acts in a complex with the coil-coiled protein ATG16 as an E3 ligase to conjugate ATG8 and PE (Figure 3). The ATG12 ATG5ATG16 complex stimulates the transfer of ATG8 from the E2-like enzyme ATG3 to PE (Hanada and Ohsumi 2005; Noda et al. 2013; Sakoh-Nakatogawa et al. 2013). Analysis of the human ATG12 protein structure showed that it binds a short peptide region of ATG3 (Metlagel et al. 2013). Only some of these residues involved in this interaction are conserved in SmATG12. However, yeast-two hybrid analysis confirmed the interaction of SmATG12 and SmATG3 (Figure 9B). In humans, a fraction of ATG3 was found to be conjugated to ATG12 via lysine 243 (K243) (Radoshevich et al. 2010), which is also conserved in SmATG3 (Supplement 2). Therefore, conjugation of SmATG3 and SmATG12 may also be of physiological relevance in S. macrospora.

Two residues responsible for mimicing a non-canonical LIR in ATG12 are conserved in SmATG12 (Figure 8) (Kaufmann et al. 2014). However, an interaction of SmATG12 and SmATG8 could not be demonstrated in the yeast-two hybrid analysis (Figure 9C). This might be explained by non-efficient lipidation of SmATG8 in yeast.

Even though $S$. macrospora SmATG12 has many conserved features, the Smatg12 cDNA was unable to complement the $S$. cerevisiae atg12 2 mutant. Interestingly, this was also the case when A. thaliana ATG12 orthologs were tested in S. cerevisiae (Hanaoka et al. 2002). We previously showed that Smatg7, Smatg8 and Smatg4 all complemented the autophagy defects of their corresponding yeast mutants (Nolting et al. 2009; Voigt and Pöggeler 2013a). The amino-acid sequence identity shared between SmATG7, SmATG8 and SmATG4 and their S. cerevisiae homologs is $53 \%, 76 \%$ and $43 \%$, respectively. In contrast, the sequence identity of SmATG12 and S. cerevisiae Atg12 is only $20 \%$. In particular, the N-terminal region of SmATG12 is conserved only among filamentous ascomycetes. Therefore, structural differences might prevent functional complementation of the yeast mutant.

\subsubsection{SmATG12 is required for sexual reproduction and normal vegetative growth}

Similar to the autophagic core components SmATG8 and SmATG4, deficiency of SmATG12 led to a reduced vegetative-growth rate and insufficient foraging under nutrient-limiting conditions, as well as the arrest of fruiting-body development at the early protoperithecium stage under nutrient-rich conditions (Figure 15) (Voigt and Pöggeler 2013a). In contrast to $\Delta$ Smatg8 and $\Delta$ Smatg4, a significant decrease in growth rate occurred only when starvation 
was induced by 3-AT (Figure 15C). Similarly, budding yeast S. cerevisiae and plant atg12 mutants exhibited reduced viability only under starvation conditions (Tsukada and Ohsumi 1993; Chung et al. 2010; Li et al. 2015).

Although, deletion of Smatg12 abolished fruiting-body formation and ascosporogenesis even under nutrient-rich conditions, we were able to isolate a homokaryotic $\Delta$ Smatg12 knockout strain from the heterokaryotic primary transformant. This demonstrates that in contrast to the E1-like enzyme SmATG7 loss of SmATG12 is not detrimental to viability or essential for ascospore germination (Nolting et al. 2009). In S. cerevisiae and the fission yeast S. pombe, atg12 deletion mutants also displayed disrupted ascosporogenesis, but only during nitrogen starvation (Tsukada and Ohsumi 1993; Mukaiyama et al. 2009). Gene expression in S. macrospora undergoes significant differences during fruiting-body development (Teichert et al. 2014), suggesting extensive de novo protein synthesis is required for fruiting-body maturation and ascospore formation. Defects in autophagy may result in a shortage of recycled amino acids and hence proteins that are critical for sexual development. Deletion of atg 12 genes in a filamentous fungus have only been reported for $N$. crassa and the plant pathogen $M$. oryzae (Kershaw and Talbot 2009; Chinnici et al. 2014). The N. crassa $\Delta$ Ncatg 12 mutant was able to initiate female development by producing ascogonia, and few small protoperithecia. Protoperithecia grafting experiments demonstrated that autophagy is required within the vegetative mycelium, because fertilized protoperithecia of $N$. crassa $\Delta$ Ncatg 12 mutants grafted onto a wild type mycelium were able to complete fruiting-body development and produced ascospores (Chinnici et al. 2014). The M. oryzae $\Delta$ Moatg12 strain was nonpathogenic and defective in appressorium formation, but effects on fruiting-body development were not reported (Kershaw and Talbot 2009). ATG12-defective plants developed normally but displayed premature senescence, produced less seeds under nutrient-rich conditions, and were hypersensitive to carbon and nitrogen starvation (Chung et al. 2010; Li et al. 2015). Similarly, knockdown of atg12 in the protozoan parasite Acanthamoeba castellanii resulted in the inhibition of cyst formation (Kim et al. 2015). Together, these results suggest that Smatg12dependent autophagy may play an important role in S. macrospora development. 


\subsubsection{Deletion of Smatg12 abolishes delivery of EGFP-labeled SmATG8 to the vacuole}

The EGFP-SmATG8 proteolysis assay revealed that SmATG12 is required for proper autophagic degradation of an EGFP-SmATG8 fusion protein (Figure 14). Furthermore, fluorescence microscopy of the functional EGFP-SmATG12 fusion protein revealed localization in bright foci and cup-shaped structures that were presumed to be the PAS and growing phagophores, respectively (Figure 16A). In S. cerevisiae and mammalian cells, ATG5 and ATG16 are markers for phagophores (also known as isolation membranes) (Mizushima et al. 2001; Mizushima et al. 2003; Suzuki et al. 2013). Both are components of the ATG12 ATG5-ATG16 complex that acts as an E3 enzyme for the efficient lipidation of ATG8 (Hanada et al. 2007). In a reconstituted in vitro system using giant unilamellar vesicles and recombinant proteins the complex was shown to tether vesicular precursors during phagophore elongation, in addition to its E3 function (Romanov et al. 2012).

In S. cerevisiae, the ATG12 ATG5-ATG16 complex is believed to be recruited to the PAS and to remain at the phagophore during expansion before detaching upon completion of autophagosome formation (Suzuki et al. 2013). In accordance with these observations, we did not observe any labeling of mature autophagosomes or vacuoles in the strain expressing EGFPSmATG12 (Figure 16A). Only a small portion of the ATG12 ATG5-ATG16 complex localizes to the PAS and the phagophore whereas the majority of the complex is reported to be localized to the cytoplasm (Mizushima 2004). Besides its function in autophagy and its association in the ATG12 ATG5-ATG16 complex, ATG12 carries out autophagy-independent roles. After conjugation to ATG3, it is involved in mitochondrial homeostasis, endosomal trafficking and apoptosis, whereas free ATG12 associates with anti-apoptotic Bcl-2 to promote mitochondrial apoptosis (Radoshevich et al. 2010; Rubinstein et al. 2011; Murrow et al. 2015). The exact localization of ATG12 during these processes remains to be determined.

When expressing EGFP-SmATG12 in an autophagy-deficient $\Delta$ Smatg8 mutant, the number of fluorescent foci appeared to increase and cup-shaped structures were not detected (Figure 16B). The enhanced localization of SmATG12 to these PAS-like structures may be explained by the blocking of phagophore extension due to the absence of SmATG8.

In the $\Delta$ Smatg12 mutant, the EGFP-SmATG8 reporter was localized to a few small dot-like structures and large fluorescent aggregates in the cytoplasm (Figure 16D). This finding is in agreement with our previous findings in which expression of EGFP-SmATG8 in a $\Delta$ Smatg4 mutant resulted in the formation of large aggregates instead of small punctate autophagosomes 
(Voigt and Pöggeler 2013a). Aggregates of a YFP-ATG8 reporter protein have been identified in a maize atg 12 mutant, and electron microscopy revealed these aggregates to be amorphous structures lacking a delineating membrane but containing the YFP-ATG8 reporter protein and ubiquitin (Li et al. 2015). In mice, ATG8 tended to aggregate in an autophagy-independent manner (Kuma et al. 2007), and autophagosomes in other organisms failed to form when components of the ATG12 ATG5-ATG16 complex were deleted (Mizushima et al. 1998; Mizushima et al. 1999; Mizushima et al. 2001; Suzuki and Ohsumi 2010). A fluorescence signal from EGFP-SmATG8 was not detected in the vacuoles of the $\Delta$ Smatg 12 mutant, indicating that ATG8-mediated autophagy was affected (Figure 16D).

In conclusion, SmATG12-mediated autophagy appears to be essential for fruiting-body formation and the production of ascospores in S. macrospora. Furthermore, SmATG12 was localized to phagophore structures in a SmATG8 dependent manner.

\subsection{The selective autophagy cargo receptor SmNBR1}

Beside the non-selective bulk autophagy, selective autophagy is characterized by cargo receptor proteins such as NBR1, which are able to bind a specific cargo including organelles, proteins or microbes for their autophagic degradation. The selective type of autophagy has not been exhaustively analyzed in filamentous fungi yet (Kimura et al. 2011; Shoji and Craven 2011; Voigt and Pöggeler 2013b; Chinnici et al. 2014).

In a study to identify new interaction partners of the core autophagy protein SmATG8 we performed a GFP-Trap analysis with EGFP-SmATG8. Among other interaction partners a putative homolog of the mammalian selective cargo receptor NBR1 (neighbor of BRCA1) was identified, which we therefore named SmNBR1 (Figure 5). Since, SmNBR1 is not encoded in S. cerevisiae and not yet analyzed in filamentous fungi we investigated its function in the ascomycete $S$. macrospora.

\subsubsection{SmNBR1 has a conserved domain architecture}

The domain architecture of SmNBR1 is conserved within filamentous ascomycetes. Aminoacid alignments revealed that the domain structure of SmNBR1 is very similar to that of orthologs of filamentous ascomycetes, including the lack of the C-terminal UBA domain, commonly found in plant and mammalian homologs, and the presence of a C-terminal conserved region, specific to fungal homologs (Figure 18). Apart from that, SmNBR1 displays many similarities to its plant and mammalian homologs, particularly in the N-terminal PB1 
domain, several ZZ-type zinc-finger domains, coiled-coil domains, the name giving NBR1 box and the C-terminal LIR motif for ATG8 interaction. The functionally uncharacterized NBR1 box domain is conserved in all NBR1-like proteins and not present in p62 (Kraft et al. 2010). The sequence identity of SmNBR1 and H. sapiens NBR1 is $22 \%$. We showed that HsnbrlDsred could be expressed in S. macrospora and it is able to partially rescue the phenotypical defects of $\Delta$ Smnbr1. However, we conclude that SmNBR1 is the homolog to the mammalian NBR1 protein. Additionally, these findings strongly support the previous phylogenetic analysis of NBR1 homolgs in eukaryotes performed by Kraft et al. (2010) where autophagy cargo receptors of filamentous ascomycetes and basidiomycetes, are characterized by the absence of UBA and other ubiquitin-binding domains (Figure 18, Figure 21).

Interestingly, the NBR1 homolog in fission yeast is characterized only by the NBR1-box, and three ZZ-type domains. In addition, the NBR1-box of the $S$. pombe NBR1 displays only a weak conservation because only two of four tryptophan residues are conserved. It lacks the $\mathrm{N}$ terminal PB1 domain and the C-terminal regions including the CR, UBA domain and the LIR motif (Mizushima 2015).

The mammalian and plant NBR1 proteins bind ubiquitinated cargos via their UBA domain. As mentioned, SmNBR1 has no obvious UBA domain and Western-blot experiments using antiubiquitin antibody revealed no distinct accumulation of ubiquitinated proteins in the $\Delta$ Smnbr1 strain. In addition to ubiquitin mediated selective autophagy, cellular cargos can be delivered for autophagy independently of ubiquitin. The Cvt pathway in yeast presents one example where the enzymes Ape1 and Ams1 are recognized by the receptors Atg19 and Atg34 to form the Cvt complex (Lynch-Day and Klionsky 2010). The Cvt pathway is not found in filamentous fungi. Similarly, the mammalian selective autophagy receptors BNIP3 (ㅁcl2/E1B $1 \underline{9} \mathrm{kDa}$ interacting protein 3), NIX (known as BNIP3L) and FUNDC1 (FUN14 domain-containing protein 1) do not use ubiquitin modifications as a degradation signal. All three receptors are mitochondrial outer membrane proteins which can directly link mitochondria to autophagosomal membranes via their LIR motif (Mazure and Pouyssegur 2010; Novak et al. 2010; Liu et al. 2012).

Analogous to the mammalian NIX, the mitochondrial anchored mitophagy receptor Atg32 links damaged mitochondria to autophagosomal membranes in an ubiquitin-independent manner in S. cerevisiae (Okamoto et al. 2009).

The pexophagy receptors Atg30 of P. pastoris and Atg36 of S. cerevisiae bind Pex3 directly at the peroxisomal membrane under peroxisome-proliferation conditions without ubiquitin (Farre 
et al. 2008). Burnett et al. (2015) identified a distinct Atg30-binding domain in P. pastoris Pex3 that modulates the phosphorylation status of the receptor and therefore its binding efficiency. Another mode of ubiquitin-independent cargo binding involves direct recognition of lipids, sugars or proteins exposed on targets. Theses signals should be presented and recognized only under starvation or stress conditions to protect cellular material from harmful components (Chu et al. 2013).

Currently, we do not know how SmNBR1 recognizes and degrades its cargo in selective autophagy. However, we may speculate that the CC domains of SmNBR1 are involved in receptor cargo interaction. All fungal NBR1 homologs contain two conserved CC domains similar to mammalian NBR1. In contrast, plant NBR1 homologs harbor no CC domains (Figure 18). Proteins containing $\mathrm{CC}$ domains play various biological roles, where the $\mathrm{CC}$ region can act as either a structural or interacting component, or both (Zhang et al. 2009; Munro 2011). For instance, the yeast cargo receptor Atg19 of the Cvt pathway specifically binds to the Ape1 protein via its CC domain (Shintani et al. 2002).

In addition to the $\mathrm{CC}$ domains fungal NBR1 homologs contain a $\mathrm{C}$-terminal $\mathrm{CR}$ motif with similarity to a C-terminal Atg11-binding site in the yeast Atg19 receptor. Both domains, CR region and the Atg11-binding site, are localized N-terminally of the LIR motif and are characterized by conserved serine residues, assumingly for phosphorylation or activation. In S. cerevisiae it was shown that the scaffold protein Atg11 binds to this serine-rich region of Atg19. Further, phosphorylation of the Atg11-binding site by the kinase Hrr25 promotes Atg19Atg11 interaction and initiates autophagosome formation (Pfaffenwimmer et al. 2014). The presence of a conserved serine-rich fungal specific CR region suggests that the yeast Atg19 protein is possibly an ortholog of fungal NBR1. Additionally, the Atg19 receptor, similar to SmNBR1, interacts with Atg8 via its LIR motif, characterized by the amino-acid motif WxxL and it does not contain a UBA domain (Noda et al. 2008; Kraft et al. 2010). Despite these common features no significant sequence homology exists between the yeast receptor Atg19 and SmNBR1 (Sawa-Makarska et al. 2014). 


\subsubsection{SmNBR1 and SmATG8 are direct interaction partners which interact via the LIR motif of SmNBR1}

All protein characteristics indicate that SmNBR1 might acts as a selective cargo receptor. Based on known cargo receptor functions, we tested the hypothesis of the involvement of SmNBR1 in autophagy, including autophagosome formation and degradation in the vacuole, by fluorescence microscopy. Cellular localization studies using functionally expressed SmNBR1EGFP/DsRED fusion proteins revealed that SmNBR1 was localized in the cytoplasm as small dots, presumably representing autophagosomes, and in vacuoles, indicating degradation of SmNBR1 (Figure 24A, Supplement 5). Similarly, localization studies of NBR1 in plants and mammals showed the formation of cytosolic bodies described as punctate structures (autophagosomes) and a localization to the central vacuole or lysosome by autophagy (Kirkin et al. 2009; Svenning et al. 2011; Zientara-Rytter et al. 2011; Zhou et al. 2013). Fluorescence microscopy studies of EGFP-SmATG8 and SmNBR1-DsRED showed co-localization of both proteins in autophagosomes-like structures and in the vacuole. We confirmed the direct interaction of SmNBR1 and SmATG8 via GFP-Trap analysis, Co-IP, yeast-two hybrid and BiFC assays. By the yeast-two hybrid analysis, we limited the region of SmNBR1 necessary for the interaction with SmATG8 to the presence of a functional LIR motif at the C-terminus. This confirms the homology of the protein to its mammalian and plant homologs (Figure 24). In mammals and plants, it has been shown that a number of cargo receptors, including NBR1, bind directly to ATG8-family proteins via the LIR motif (Pankiv et al. 2007; Ichimura et al. 2008; Johansen and Lamark 2011; Svenning et al. 2011). For mammalian NBR1, it was found that the LIR motifs are the major interaction surface for interaction with ATG8-like proteins, as mutations in the LIR motif caused a failure in the interaction with ATG8 (Kirkin et al. 2009; Lamark et al. 2009). Furthermore, yeast-two hybrid interaction studies of human NBR1 and ATG8 family proteins provide a detailed explanation of how the C-terminal sequence (aa 691966) of NBR1 can interact with ATG8 (Kirkin et al. 2009). Studies using yeast-two hybrid analysis, pull-down experiments and mutation analysis with the plant homologs A. thaliana NBR1 and Nicotiana tabacum Joka2 also showed that NBR1-ATG8 interaction is limited to the LIR motif (Svenning et al. 2011; Zientara-Rytter et al. 2011). 


\subsubsection{The $S$. macrospora SmNBR1 is required for proper sexual development, vegetative growth and stress resistance}

Autophagy plays diverse roles in biological processes of filamentous ascomycetes including sexual development, viability and vegetative growth (Voigt and Pöggeler 2013b; Teichert et al. 2014). The putative $S$. macrospora homolog of the selective autophagy cargo receptor NBR1 is also involved in developmental processes as well as starvation and stress response in S. macrospora.

Initially, the Smnbrl knockout strain showed defects in late stages of fruiting-body development and displayed an impaired vegetative growth. The mutant formed ascogonia, protoperithecia and perithecia but in a reduced number and after a prolonged time. The ascus rosettes of the mutant harbored incomplete asci with few mature ascospores (Figure 20). Consequently, the number of discharged mature ascospores, which are essential for reproduction, was also drastically reduced. These effects are more pronounced under starvation and stress-inducing conditions when compared to the wild type. In S. macrospora, studies of autophagy-deficient $\Delta$ Smatg8, $\Delta$ Smatg4 and $\Delta$ Smatg 12 mutants displayed a sterile phenotype in combination with defects in vegetative growth and starvation intolerance and deletion of Smatg7 is even lethal (Nolting et al. 2009; Voigt and Pöggeler 2013a; Werner et al. 2016). Previous analysis of the selective autophagy process in A. thaliana showed that disruption of the nbrl gene caused impairment in autophagosome formation and the mutant was hypersensitive to oxidative, drought and salt stresses. The $\Delta$ Atnbr1 as well as the tomato NBR1a/NBR1b silencing strain showed an increased sensitivity to heat stress by accumulating ubiquitinated substrates in cells. Autophagy-defective mutant- or transgenic plants showed extensive wilting and drought stress symptoms and seedlings were not salt tolerant. A knockout of the NBR1 homolog Joka2 in N. tabacum, could not be generated but analysis showed an apparently induction of the gene expression of JOKA2 and ATG8 in the roots when plants were grown in either N- or S-deficiency (Zientara-Rytter et al. 2011; Zhou et al. 2013; Zhou et al. 2014a). Similarly, in S. macrospora deletion of the Smnbrl caused increased sensitivity to starvation and stress conditions, and impaired development of sexual structures.

To determine whether the role of SmNBR1 in starvation and stress conditions is due to its action as an autophagy cargo receptor, we performed genetic complementation of $\Delta$ Smnbr 1 with the wild type Smnbrl gene (ectopically integrated). Transformants expressing the wild type Smnbrl gene almost fully restored the starvation and stress tolerance of the mutant (Figure 28). Interestingly, complementation analysis of $\Delta$ Smnbr1 with truncated versions of SmNBR1 could 
not improve the formation of perithecia and mature ascospores. These results indicate that a complete SmNBR1 protein is required for developmental processes and sexual spore production but this is just partly true for mutated versions of SmNBR1. The SmNBR1 versions without or a mutated LIR motif including the residues $\mathrm{T}^{843} \mathrm{~A}, \mathrm{Y}^{847} \mathrm{~A}$ and $\mathrm{L}^{850} \mathrm{~A}$, have not the ability to fully complement the $\Delta$ Smnbrl defects. Therefore, it can be concluded that these residues are important for the function of the LIR motif. The exchange of the serine residues to alanine residues in the conserved region showed less defects in complementation strains, leading to the assumption that the CR domain is not essential for SmNBR1 function.

In autophagy-defective $\Delta \operatorname{Smatg} 8, \Delta \operatorname{Smatg} 4$ and $\Delta$ Smatg1 2 strains, SmNBR 1 forms cytoplasmic aggregates but is not delivered to the vacuole. Therefore, damaged and useless organelles or cellular proteins might be still targeted by SmNBR1 but not degraded in an autophagydependent manner and therefore accumulate at high levels as insoluble cytoplasmic aggregates (Figure 25A). The same has been shown in autophagy-defective plants and mammals, where endogenous AtNBR1 and NBR1, respectively, accumulated in the cytoplasm (Goldberg 2003; Komatsu et al. 2006; Kirkin et al. 2009; Svenning et al. 2011).

\subsubsection{SmNBR1 has many putative phosphorylation sites}

Selective autophagy pathways are regulated by phosphorylation. In S. cerevisiae, the cargo receptors Atg30, Atg32, Atg36, Atg34 and Atg19 are phosphorylated under mito- and pexophagy-inducing conditions (Farre et al. 2013; Kanki et al. 2013). While the kinase CK2 regulates Atg32 function, the kinase Hrr25, a homolog of casein kinase $1 \delta(\mathrm{CK})$, phosphorylates Atg19, Atg34 and Atg36 to initiate the Cvt pathway and pexophagy, respectively, by promoting their interaction with Atg11 (Pfaffenwimmer et al. 2014; Tanaka et al. 2014).

In mammals, random bulk autophagy is regulated by the phosphoinositide $\underline{3}$-kinase (PI3K). Selective autophagy is controlled by phosphorylation of the cargo receptor SQSTM1/p62 by CK2, which increases the affinity of the receptor to polyubiquitin chains and consequently to target proteins for their autophagic degradation (Matsumoto et al. 2011). Moreover, the mammalian NBR1 protein is phosphorylated at $\mathrm{T}^{586}$ by kinase GSK3, which regulates its function as cargo receptor (Nicot et al. 2014).

The program NetPhos3.1 predicted 113 potential phosphorylation sites in SmNBR1 comprising 56 serine, 45 threonine and 12 tyrosine residues. These include amino-acid residues $\mathrm{T}^{843}$ and $\mathrm{Y}^{847}$ of the LIR motif, which were predicted to be substrates of the kinases CK2 and SRC, respectively. Additionally, the residues $S^{793 / 804 / 808}$ of the conserved region could be identified 
as substrates of the kinases DNAPK (phosphatidylinositol-3-kinase related kinase) and GSK3. Other putative sites were predicted to be targets of CK2 or the cyclin dependent kinase 5 (CDK5).

By Western-blot analysis, several bands of SmNBR1-DsRED were detected, using an anti-RFP antibody suggesting that SmNBR1 is indeed subjected to posttranslational modifications. Moreover, phosphatase treatment reduced the number of additional bands in the Western blot (Figure 27). Thus, it can be assumed that SmNBR1 is modified by phosphorylation similar to other NBR1 homologs or other cargo receptors active in selective autophagy processes. Similarly, in mammals and plants phosphorylated NBR1 often migrates at other sizes than their predicted molecular weight. Commercial antibodies targeted against mammalian full-length NBR1 mostly recognize several bands around $150 \mathrm{kDa}$ despite a theoretical size of $108 \mathrm{kDa}$ for the longest isoform of NBR1 (Kirkin et al. 2009; Nicot et al. 2014). The same result is observable for the plant NBR1 homolog which shows several bands in Western-blot experiments (Zhou et al. 2013).

\subsubsection{In S. macrospora, pexophagy depends on SmNBR1}

Beside the fact that peroxisomes are essential for fatty acid $\beta$-oxidation and detoxification of reactive oxygen species (ROS) they have special functions in filamentous fungi. Fungal mutants lacking peroxisomes fail to grow on media containing fatty acids as sole carbon source (Erdmann et al. 1989; Hynes et al. 2008). Peroxisomes are also responsible for the biosynthesis of biotin and the formation of secondary metabolites including the biosynthesis of penicillins, polyketides, terpenes and paxilline (Maggio-Hall et al. 2005; Saikia and Scott 2009; Imazaki et al. 2010; Bartoszewska et al. 2011; Tanabe et al. 2011). Additionally, peroxisomes are involved in plant pathogenicity (Kimura et al. 2001; Asakura et al. 2006), sexual development and growth (Bonnet et al. 2006; Managadze et al. 2007; Peraza-Reyes et al. 2008).

The selective degradation of peroxisomes via autophagy depends in P. pastoris on Atg30 and in S. cerevisiae on Atg36 (Farre et al. 2008; Motley et al. 2012; Tanaka et al. 2014), but homologs of these proteins are not present in S. macrospora (Table 1). The P. pastoris cargo receptor Atg30 localizes to the peroxisomal membrane by binding to Pex3 and Pex14. It gets phosphorylated and upon induction it interacts with core autophagy components Atg11, Atg17 and Atg8. As additional protein Atg37 is involved in the assembly of a receptor protein complex. It has been suggested that the acyl-CoA binding protein Atg37 facilitate the Atg30Atg11 interaction (Nazarko et al. 2014). In S. cerevisiae, Atg36 is recruited to peroxisomes by 
Pex3 and is specifically required for pexophagy by indirect binding to Atg8 with the help of the scaffold protein Atg11 (Figure 39A).

In plants, it is speculated that NBR1 plays a role in pexophagy during heat stress because an over-accumulation of catalases in aggregates of an A. thaliana Atnbr1 mutant could be observed (Zhou et al. 2014b; Young and Bartel 2016). However, the direct involvement of plant NBR1 in pexophagy was not experimentally verified so far. It is speculated that a selective autophagy receptor binds to the peroxisome as well as to ATG8, thus connecting the organelle to the autophagy machinery. The binding signal, which is recognized by the selective receptor, has not been identified yet but candidates include ubiquitinated proteins such as PEX5 or a matrix protein such as PEX3 or PEX14 (Young and Bartel 2016) (Figure 39B).

Further, NBR1 and its partner p62 are involved in the selective degradation of peroxisomes in mammals (Kirkin et al. 2009; Deosaran et al. 2013). It was shown, that NBR1 is necessary and sufficient for pexophagy to maintain a healthy peroxisome population (Deosaran et al. 2013). In mammals, three different types of peroxisome degradation have been reported so far (HaraKuge and Fujiki 2008; Kim et al. 2008; Deosaran et al. 2013; Yamashita et al. 2014). First, pexophagy can be induced by recognition of a ubiquitinated unknown peroxisomal protein by p62, which bridges the ubiquitin-decorated peroxisome with the developing autophagosomes via LC3-II (ATG8) (Kim et al. 2008). Secondly, under nutrient-starvation conditions the peroxisomal membrane protein PEX14 prefers to bind directly to LC3-II (ATG8) in a competitive manner with PEX5 (Hara-Kuge and Fujiki 2008) (Figure 39C). Thirdly, Deosaran et al. (2013) showed that pexophagy is also induced by overexpression of NBR1, which promotes peroxisome clustering around NBR1 and autophagic peroxisome degradation. Moreover, mono-ubiquitinated PEX5 and overexpression of PEX3 target NBR1 to clustered peroxisomes (Deosaran et al. 2013; Yamashita et al. 2014). 


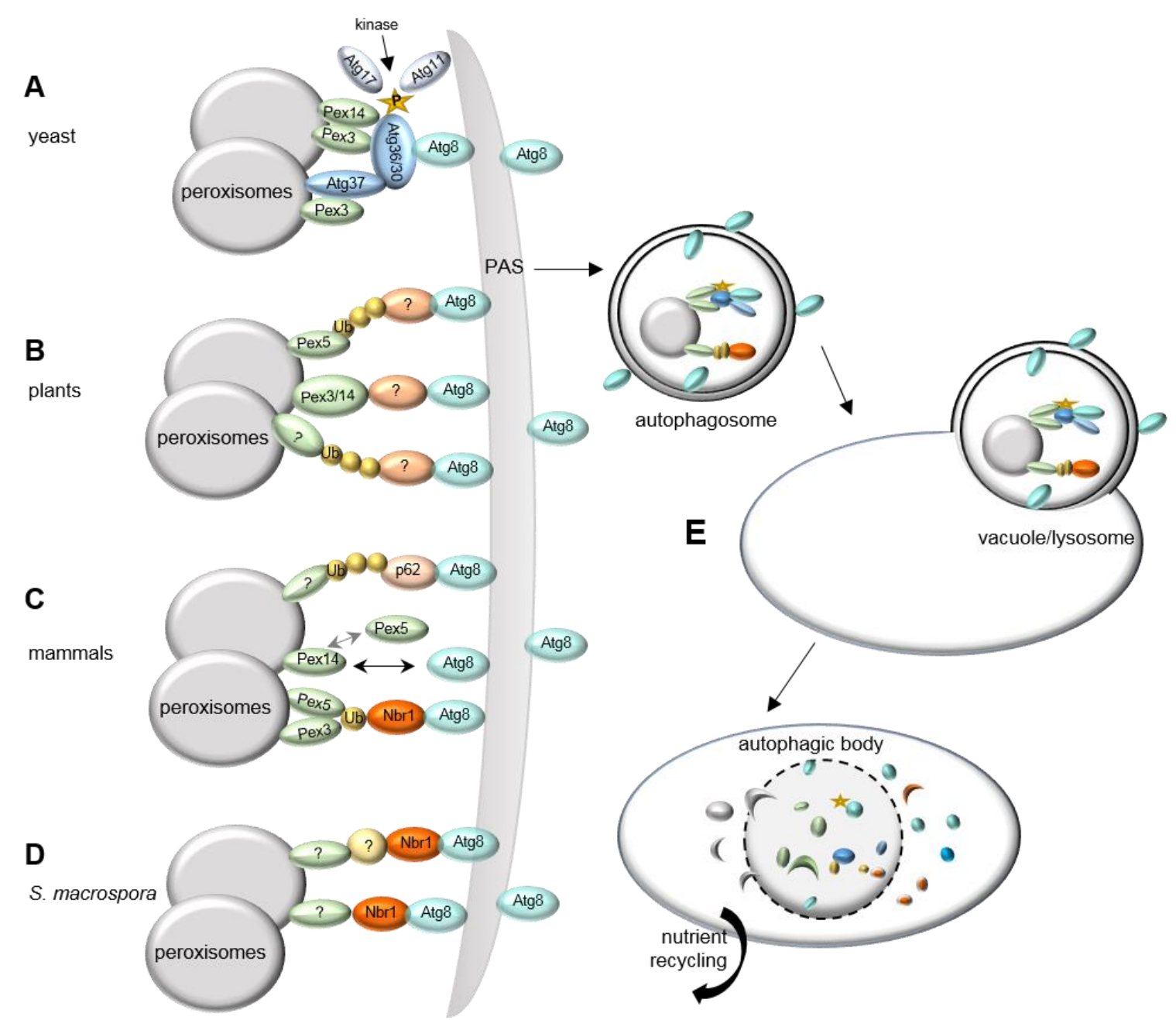

Figure 39: Mechanistic views of receptor-dependent pexophagy pathways.

(A) The phosphoprotein Atg30, identified in the yeast P. pastoris, is the receptor for pexophagy. This protein can interact with the peroxisomal membrane protein Pex3/Pex14 and/or Atg37 and becomes phosphorylated during pexophagy (yellow star). Atg30 interacts with the autophagy-adapter proteins Atg11 and Atg17 at the pexophagy specific PAS. Together with Atg8 it is required for the formation of the phagophore membrane that engulfes peroxisomes. Another receptor is Atg36 identified in S. cerevisiae. Atg36 is anchored to the peroxisome by its association with Pex3 and after phosphorylation it also interacts with Atg11 and Atg8. (B) In plants, it is postulated that an autophagy cargo receptor is bind to the peroxisome and to Atg8, connecting the degrading peroxisome to the autophagy machinery. The signal on plant peroxisomes for the selective autophagy receptor has not been identified. Candidates include ubiquitinated proteins such as the peroxisomal targeting receptor Pex 5 or membrane proteins Pex3 or Pex14. (C) Peroxisomes can be targeted by p62-mediated detection of ubiquitin motifs on still unknown peroxisomal membrane proteins or by a direct binding of Atg8 (LC3-II) to Pex14 in a Pex5-competitive manner. When the mammalian peroxisomal import receptor Pex5 is recruited to the peroxisomal membrane ubiquitination takes place. The mono-ubiquitinated Pex5 is a target of Nbr1, which in turn interacts with Atg8 at the PAS. Additionally, overexpression of Pex3 can target NBR1 to clustered peroxisomes. (D) In S. macrospora, the hyphothetical mechanism of peroxisome targeting is dependent on a selective cargo receptor, possibly SmNBR1 (Nbr1), which interacts with Atg8 at the PAS. But the target protein at the peroxisomal membrane is unknown. SmNBR1 may either directly target a peroxisomal membrane protein or an additional protein is interposed. (E) The phagophore completely encloses its cargo forming an autophagosomes, which is delivered to the vacuole or lysosome to form the autophagic body. The whole content is degraded and building blocks are released into the cytosol for reuse. Ub, ubiquitin; PAS, phagophore assembly site; P, phosphorylation.

Detailed studies of the mammalian NBR1 demonstrated that the amphipathic $\alpha$-helical J domain (JUBA), the ubiquitin-binding UBA domain, the LIR motif and the CC domain are essential to mediate pexophagy. NBR1 uses a mechanism where the combination of the UBA domain and 
the membrane interacting $\mathbf{J}$ domain are involved, to bind to the peroxisomal lipid bilayer (Deosaran et al. 2013).

The plant NBR1 homologs from N. tabacum and A. thaliana contain UBA1 and UBA2 domains (Figure 18). It has been shown that only UBA2 can bind ubiquitin in vitro (Svenning et al. 2011) and the UBA1 domain is capable to form an amphipathic helix. Therefore, it was attempting to speculate that this region might have a similar role as the JUBA domain of human NBR1 (Zientara-Rytter et al. 2011). However, it seems that the JUBA domain is specific for animal NBR1 (Mardakheh et al. 2010) because a similar domain could not be found in plants and fungi. The NBR1 homolog in $S$. macrospora is an attractive fungal candidate for a pexophagy receptor (Figure 39D). In this study we demonstrated that the S. macrospora $\Delta$ Smnbr1 strain is not able to use fatty acids as sole carbon source and $\Delta$ Smnbr1 is not able to form fruiting bodies on medium containing $\mathrm{H}_{2} \mathrm{O}_{2}$ (Figure 29). Therefore, it may be suggested that SmNBR1 is involved in the turnover of viable peroxisomes. By fluorescence microscopy it was shown that the degradation of the peroxisomal reporter protein DsRED-SKL is arrested in $\triangle \mathrm{Smnbr} 1$ compared to the wild type (Figure 30). The peroxisomal marker protein SmPEX14 was additionally analyzed by fluorescence localization studies in wild type, $\Delta$ Smnbr1 and $\Delta$ Smatg8 strains. When compared to the wild type, peroxisomes changed in size, number and morphology in $\Delta$ Smnbrl and $\Delta$ Smatg8 strains (Figure 31 ). The protein or signal that labels peroxisomes for their degradation is not identified in S. macrospora so far. SmNBR1 contains a LIR motif for binding to SmATG8 but the UBA domain is missing for binding to ubiquitin or an ubiquitinated substrate as described for pexophagy in mammals.

\subsubsection{Woronin bodies are functional in autophagy-defective mutants}

Woronin bodies are peroxisome-derived organelles of filamentous fungi. They function as a plug of septal pores after hyphal wounding to prevent the loss of cytoplasm at sites of injury. The dominant protein of this organelle is HEX1 which forms hexagonal crystals in vitro and is localized to the matrix of the Woronin bodies in large and stable complexes (Jedd and Chua 2000). Deletion of HEX1 has been described to lead to impairment of Woronin body biogenesis and results in diverse effects in cellular and developmental processes. In N. crassa, A. oryzae and $M$. grisea, hexl deletion strains show extensive cytoplasmic lost after hyphal lesions. In N. crassa, HEX1 is described as a very stable oligomeric structure (Jedd and Chua 2000; Tenney et al. 2000; Soundararajan et al. 2004). 
Our findings suggest that Woronin bodies are still present and functional in tested autophagydefective strains. Microscopic analysis using SmHEX1 in $\Delta$ Smnbr1 and $\Delta$ Smatg8 mutants confirmed the presence of these organelles. In the Western blot several bands of DsREDSmHEX1 suggested that different oligomers of the protein exist in the mutants and in wild type (Figure 33). Fluorescence microscopic analysis revealed that the DsRED-SmHEX1 reporter protein localizes to septal pores and Woronin bodies which plug septal pores after injury in wild type and the $\Delta$ Smnbr1 mutant (Figure 33). Thus, selective degradation of Woronin bodies seems to be not affected in $\Delta$ Smnbr1. Furthermore, it can be assumed that Woronin bodies and HEX1 protein are very stable and have a low turnover rate. Therefore, it will be difficult to established assays for "Woroniphagy".

\subsubsection{SmNBR1 is involved in selective degradation of the large ribosomal subunit}

The present study supports the notion that ribosomal subunits are not only degraded by bulk autophagy, but also selective autophagic ribosomal turnover takes place in the ascomycete S. macrospora. It is known that ribosomes constitute half of the cell's protein mass (Warner 1999) and consequently, represent a major source of amino acids during times of nutrient deprivation and adjustment to stress-inducing conditions. In addition, ribosomal degradation during starvation might be important for the rapid and simultaneous downregulation of protein translation, a process that consumes large amounts of energy and amino acids. Mature ribosomes are randomly sequestered into autophagosomes and degraded in the vacuole during non-selective autophagy. On the other hand, reports in S. cerevisiae suggest that under specific conditions a cargo receptor binds to ribosomal subunits followed by their exclusive enwrapping into double-membrane vesicles and their subsequent selective degradation via ribophagy (Kraft et al. 2008).

Observations in S. cerevisiae have revealed that the large $60 \mathrm{~S}$ ribosomal subunits are more rapidly degraded compared to other cytoplasmic components, supporting the idea of a selective degradation process. The involvement of autophagy in this process was demonstrated by uncovering that the transport of ribosomes to the vacuole relies on core autophagy components such as Atg1 and Atg7 (Kraft et al. 2008). A genetic screen in yeast designed to isolate mutant strains with a defect in ribosome turnover revealed that the ubiquitin protease Ubp3 and its cofactor Bre5 are required for this selective type of autophagy, however, not for bulk autophagy (Figure 40A). Under nutrient-rich conditions, the ubiquitin ligase Ltn1 ubiquitinates the 60S 
ribosomal subunit protein Rpl25 and protects it from degradation by autophagy, whereas under starvation conditions deubiquitination of Rpl25 by the deubiquitinase complex Ubp3/Bre5 induces degradation of the large $60 \mathrm{~S}$ ribosomal subunit. It was suggested that deubiquitinated Rpl25 is the target of a ribophagy cargo receptor (Kraft et al. 2008; Ossareh-Nazari et al. 2014). However, the selective receptor for ribosome-autophagosome assembly was not yet been identified in S. cerevisiae.

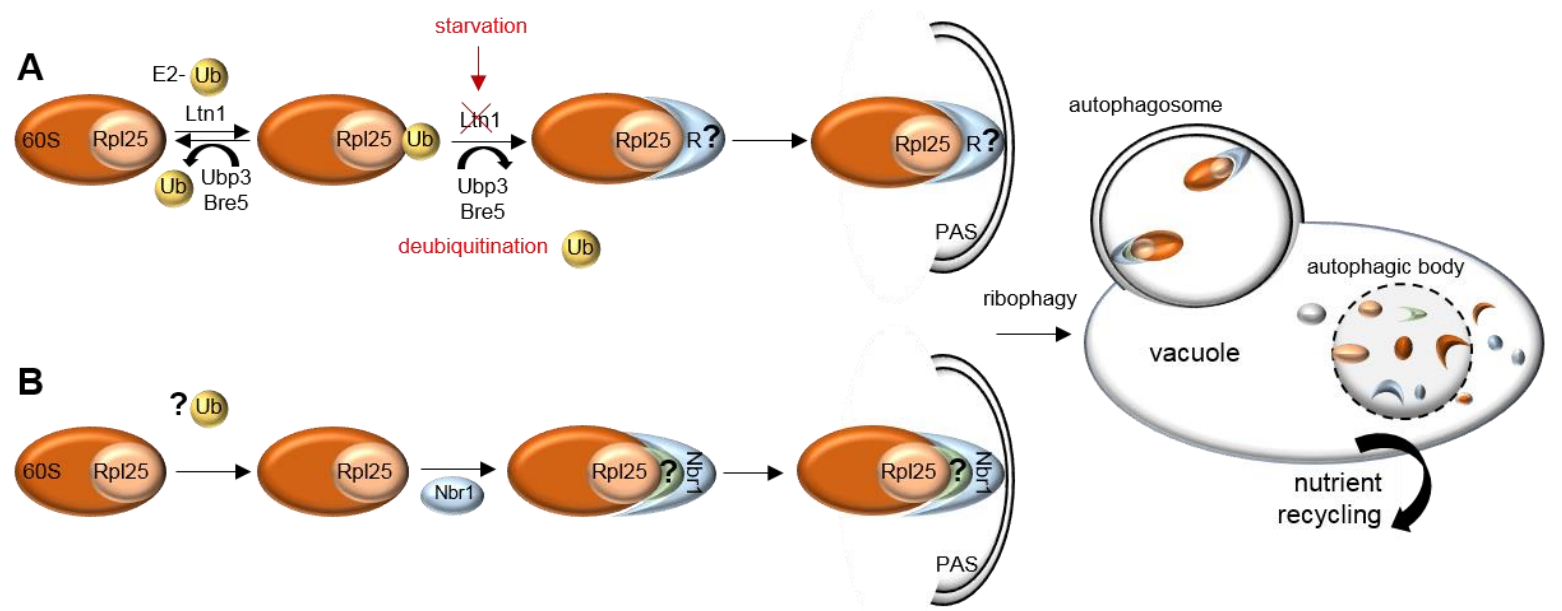

Figure 40: Hypothetical model for the ribophagy of the large ribosomal subunit in S. cerevisiae and S. macrospora.

(A) In S. cerevisiae, under nutrient-rich conditions the E3 ligase Ltn1 ubiquitinates Rp125 from the large 60S ribosomal subunit. In contrast, under starvation conditions the level of Ltn1 decreases and the Ubp3/Bre5 deubiquitinase complex acts in the deubiquitination of Rpl25. Rpl25 is targeted by a still unknown receptor for selective autophagy. (B) It is hypothesized that in S. macrospora SmNBR1 (Nbr1) binds selectively to a protein of the large ribosomal subunit or another yet unknown protein to bridge the 60S subunit including SmRPL25 (Rp125) to the PAS. The autophagosome encloses the cargo, which is in turn delivered to the vacuole to form the autophagic body. The whole content is degraded into their constitutive nutrients and released into the cytosol for reuse. 60S, large ribosomal subunit; Ub, ubiquitin; PAS, phagophore assembly site.

In S. macrospora, the deletion of Smnbrl results in a defect in the turnover of the large ribosomal subunit protein SmRPL25-EGFP shown by quantitative Western-blot experiments and fluorescence localization studies (Figure 34). Importantly, SmATG8 is normally degraded by autophagy in this deletion strain (Figure 37). The transport of SmRPL25-EGFP to the vacuole relies on the core autophagy protein SmATG8 because SmRPL25-EGFP is not degraded in a $\Delta$ Smatg8 strain. Additionally, the interaction of SmNBR1 and the 60S ribosomal subunit protein SmRPL25 could be verified in vivo by immunoprecipitation (Figure 38). However, a yeast-two hybrid analysis revealed that SmRPL25 is no direct interaction partner of SmNBR1 (Supplement 8).

The analysis of the small ribosomal subunit protein SmRPS19 shows the same localization as SmRPL25 in fluorescence microscopy. In the wild type the EGFP-tagged SmRPS19 is degraded in the vacuole whereas a stabilization of SmRPS19-EGFP is visible in $\triangle$ Smnbr1 and 
the autophagy-defective mutant $\Delta$ Smatg8 (Figure 35A,C). Surprisingly, the quantitative Western-blot analysis of SmRPS19-EGFP revealed no significant differences in stability when the wild type and the $\Delta$ Smnbrl strain were compared under rich and starvation conditions (Figure 35B,D). However, it has to be considered that the time point of fluorescence microscopic analysis differs from the time point of the Western-blot quantification. Due to technical reasons for microscopic localization studies, strains were analyzed after 18-72 $\mathrm{h}$ of growth whereas proteins for the Western-blot analysis were sampled after 3-6 days of growth. Therefore, it might be that after a certain time of growth stability of ribosomal proteins reach a steady state level that do not allow to detect further differences in stability. Therefore, it is not clear that the degradation of SmRPS19 is realy independent of the selective receptor SmNBR1. Nevertheless, similar to SmRPL25, SmRPS19-EGFP was stabilized in the autophagy-defective mutant $\Delta$ Smatg8, which indicates that also the small ribosomal subunit was degraded in an autophagy-dependent manner.

In the GFP-Trap analysis using SmNBR1 as bait protein many ribosomal associated proteins were identified by LC/MS analysis as putative interaction partners (Table 7). Astonishingly, the interaction partner of SmNBR1 identified with the highest significance was the small ribosomal subunit protein SmRPS27. Additionally, SmRPS21 and one large ribosomal subunit protein SmRPL28 were identified among the eight best hits. Moreover, SmRPS26 was identified as the $16^{\text {th }}$ best hit (data not shown).

A BLASTP search revealed that SmRPL28 is not encoded in S. cerevisiae and other yeasts. The RPL28 of S. macrospora is a homolog of the human and Drosophila melanogaster L28e protein, which is lacking in yeast (Anger et al. 2013). Another interesting candidate among the eight best hits is the ribosome-associated protein SmSTM1 which has been shown to stabilize ribosomes during starvation conditions (Van Dyke et al. 2006; Ben-Shem et al. 2011; Jenner et al. 2012). SMAC_04779 (fatty acid degradation enzyme), SMAC_02849 (amino-acid biosynthesis) and SMAC_00402 (pyrimidine metabolism) among the best eight hits are predicted to have enzymatic activities in general metabolic processes. BLASTP searches at UniProt (http://www.uniprot.org/blast/uniprot/) revealed that these metabolic proteins are conserved among ascomycetes.

Figure 41 represents the crystal structure of the $80 \mathrm{~S}$ ribosome of human with highlighted ribosomal subunit proteins identified in GFP-Trap analysis, including RPS21, 26, 27 and RPL28, as well as the proteins used for stability analysis, RPS19 and RPL25. In this model the small 40S ribosomal subunit proteins identified in GFP-Trap analysis (RPS21, 26, 27) localize on a different position as the RPS19 protein which was tested for SmNBR1-dependent 
degradation. Also RPL25, which was assayed in the ribophagy analysis, is localized apart from RPL28 in the crystal structure. Therefore, it is not surprisingly that RPL25 was not identified as a direct interaction partner of SmNBR1 in the yeast-two hybrid analysis. The ribosomal proteins and the ribosomal-associated protein STM1 identified in the GFP-Trap analysis are therefore excellent canditates to test SmNBR1-dependent ribophagy in the future.

A

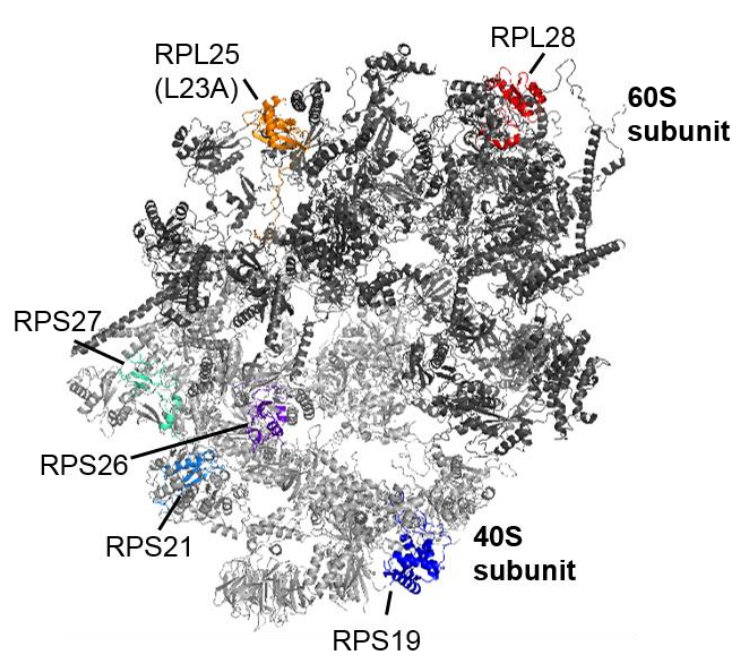

B $\quad 80$ S human ribosome + rRNA

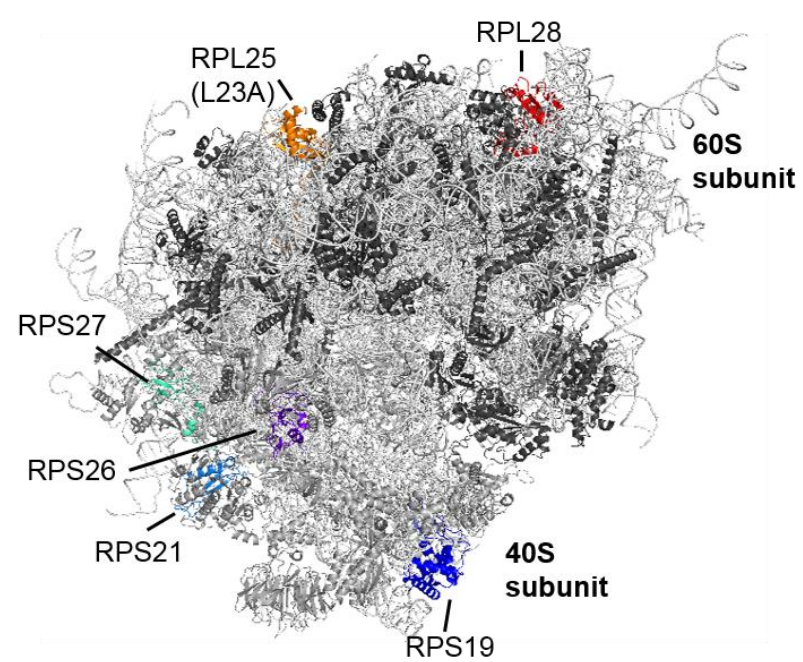

Figure 41: Crystal structure of the 80S human ribosome and ribosomal RNA (Anger et al. 2013).

(A) Molecular model of the $80 \mathrm{~S}$ human ribosome consists of the $60 \mathrm{~S}$ subunit (dark grey) and the $40 \mathrm{~S}$ subunit (light grey) in cartoon representation (PDB ID: 4V6X). Ribosomal proteins are coloured individually. The large 60S ribosomal subunit proteins RPL25 (known in human as L23A (Ban et al. 2014)) is labeled in orange and RPL28 is labeled in red. The small 40S ribosomal subunit proteins RPS27 is labeled in cyan, RPS26 in purple, RPS21 in light blue and RPS19 is labeled in dark blue. (B) 80S human ribosome with rRNA shown in white as cartoon representation (PDB ID: 3J3D, 3J3F).

The results demonstrate that functional SmNBR1 is required to promote the selective degradation of mature $60 \mathrm{~S}$ ribosomal subunits. It can be speculated that under starvation conditions SmNBR1 acts as cargo receptor to bind to the $60 \mathrm{~S}$ ribosomal subunit and recruits surplus ribosomal subunits to autophagosomes for their degradation in the vacuole. The direct target of SmNBR1 is so far unknown. Ribosomal proteins identified in the GFP-Trap analysis might be the targets of SmNBR1 or alternatively a yet unknown protein. It is rather unlikely that ubiquitin or deubiquitination are involved in this process (Figure 40B) since ubiquitin was not identified as SmNBR1-interacting protein. Further, SmNBR1 does not exhibit an obvious UBA domain and $\Delta$ Smnbr1 showed no accumulation of ubiquitinated proteins.

In summary, the findings of this thesis imply that SmNBR1 is a homolog of the mammalian autophagy cargo receptor NBR1. Moreover, the results reveal that the selective recognition and binding of excessive, damaged or non-functional ribosomes as well as peroxisomes are crucial for vegetative growth under stress conditions and for sexual development in $S$. macrospora. 


\section{References}

Alemu EA, Lamark T, Torgersen KM, Birgisdottir AB, Larsen KB, Jain A, Olsvik H, Overvatn A, Kirkin V, Johansen T. 2012. ATG8 family proteins act as scaffolds for assembly of the ULK complex: sequence requirements for LC3-interacting region (LIR) motifs. The Journal of biological chemistry 287: 39275-39290.

Anger AM, Armache JP, Berninghausen O, Habeck M, Subklewe M, Wilson DN, Beckmann R. 2013. Structures of the human and Drosophila 80S ribosome. Nature 497: 80-87.

Araki Y, Ku WC, Akioka M, May AI, Hayashi Y, Arisaka F, Ishihama Y, Ohsumi Y. 2013. Atg38 is required for autophagy-specific phosphatidylinositol 3-kinase complex integrity. J Cell Biol 203: 299-313.

Asakura M, Okuno T, Takano Y. 2006. Multiple contributions of peroxisomal metabolic function to fungal pathogenicity in Colletotrichum lagenarium. Appl Environ Microb 72: 6345-6354.

Avin-Wittenberg T, Honig A, Galili G. 2012. Variations on a theme: plant autophagy in comparison to yeast and mammals. Protoplasma 249: 285-299.

Ban N, Beckmann R, Cate JHD, Dinman JD, Dragon F, Ellis SR, Lafontaine DLJ, Lindahl L, Liljas A, Lipton JM et al. 2014. A new system for naming ribosomal proteins. Curr Opin Struc Biol 24: 165-169.

Bartoszewska M, Opalinski L, Veenhuis M, van der Klei IJ. 2011. The significance of peroxisomes in secondary metabolite biosynthesis in filamentous fungi. Biotechnol Lett 33: 1921-1931.

Behrends C, Fulda S. 2012. Receptor proteins in selective autophagy. International journal of cell biology 2012: 673290 .

Ben-Shem A, de Loubresse NG, Melnikov S, Jenner L, Yusupova G, Yusupov M. 2011. The structure of the eukaryotic ribosome at 3.0 angstrom resolution. Science 334: 15241529.

Birnboim HC, Doly J. 1979. A rapid alkaline extraction procedure for screening recombinant plasmid DNA. Nucleic acids research 7: 1513-1523.

Bloemendal S, Bernhards Y, Bartho K, Dettmann A, Voigt O, Teichert I, Seiler S, Wolters DA, Pöggeler S, Kück U. 2012. A homologue of the human STRIPAK complex controls sexual development in fungi. Mol Microbiol 84: 310-323.

Bonnet C, Espagne E, Zickler D, Boisnard S, Bourdais A, Berteaux-Lecellier V. 2006. The peroxisomal import proteins PEX2, PEX5 and PEX7 are differently involved in Podospora anserina sexual cycle. Mol Microbiol 62: 157-169.

Brew CT, Huffaker TC. 2002. The yeast ubiquitin protease, Ubp3p, promotes protein stability. Genetics 162: 1079-1089.

Brocard C, Hartig A. 2006. Peroxisome targeting signal 1: Is it really a simple tripeptide? BbaMol Cell Res 1763: 1565-1573.

Burnett SF, Farre JC, Nazarko TY, Subramani S. 2015. Peroxisomal Pex3 activates selective autophagy of peroxisomes via interaction with the pexophagy receptor Atg30. Journal of Biological Chemistry 290: 8623-8631.

Cao Y, Cheong HS, Song H, Klionsky DJ. 2008. In vivo reconstitution of autophagy in Saccharomyces cerevisiae. J Cell Biol 182: 703-713.

Carroll AM, Sweigard JA, Valent B. 1994. Improved vectors for selecting resistance to hygromycin. Fungal Genet Newslett 41: 22.

Chinnici JL, Fu C, Caccamise LM, Arnold JW, Free SJ. 2014. Neurospora crassa female development requires the PACC and other signal transduction pathways, transcription factors, chromatin remodeling, cell-to-cell fusion, and autophagy. Plos One 9: e110603. 
Christianson TW, Sikorski RS, Dante M, Shero JH, Hieter P. 1992. Multifunctional yeast highcopy-number shuttle vectors. Gene 110: 119-122.

Chu CT, Ji J, Dagda RK, Jiang JF, Tyurina YY, Kapralov AA, Tyurin VA, Yanamala N, Shrivastava IH, Mohammadyani D et al. 2013. Cardiolipin externalization to the outer mitochondrial membrane acts as an elimination signal for mitophagy in neuronal cells. Nature Cell Biology 15: 1197-2005.

Chung T, Phillips AR, Vierstra RD. 2010. ATG8 lipidation and ATG8-mediated autophagy in Arabidopsis require ATG12 expressed from the differentially controlled ATG12A AND ATG12B loci. The Plant Journal 62: 483-493.

Colot HV, Park G, Turner GE, Ringelberg C, Crew CM, Litvinkova L, Weiss RL, Borkovich KA, Dunlap JC. 2006. A high-throughput gene knockout procedure for Neurospora reveals functions for multiple transcription factors. Proceedings of the National Academy of Sciences of the United States of America 103: 10352-10357.

Cox J, Neuhauser N, Michalski A, Scheltema RA, Olsen JV, Mann M. 2011. Andromeda: a peptide search engine integrated into the MaxQuant environment. J Proteome Res 10: 1794-1805.

Cuervo AM. 2004. Autophagy: in sickness and in health. Trends in cell biology 14: 70-77.

Deosaran E, Larsen KB, Hua R, Sargent G, Wang Y, Kim S, Lamark T, Jauregui M, Law K, Lippincott-Schwartz J et al. 2013. NBR1 acts as an autophagy receptor for peroxisomes. Journal of cell science 126: 939-952.

Distel B, Erdmann R, Gould SJ, Blobel G, Crane DI, Cregg JM, Dodt G, Fujiki Y, Goodman JM, Just WW et al. 1996. Unified nomenclature for peroxisome biogenesis factors. $J$ Cell Biol 135: 1-3.

Effelsberg D, Cruz-Zaragoza LD, Schliebs W, Erdmann R. 2016. Pex9p is a novel yeast peroxisomal import receptor for PTS1-proteins. Journal of cell science.

Elleuche S, Pöggeler S. 2008. Visualization of peroxisomes via SKL-tagged DsRed protein in Sordaria macrospora. Fungal Genetics Reports 55: 8-12.

-. 2009. $\beta$-carbonic anhydrases play a role in fruiting body development and ascospore germination in the filamentous fungus Sordaria macrospora. Plos One 4: e5177.

Engh I, Nowrousian M, Kück U. 2010. Sordaria macrospora, a model organism to study fungal cellular development. European journal of cell biology 89: 864-872.

Epple UD, Suriapranata I, Eskelinen EL, Thumm M. 2001. Aut5/Cvt17p, a putative lipase essential for disintegration of autophagic bodies inside the vacuole. $J$ Bacteriol 183: 5942-5955.

Erdmann R, Schliebs W. 2005. Peroxisomal matrix protein import: the transient pore model. Nat Rev Mol Cell Bio 6: 738-742.

Erdmann R, Veenhuis M, Mertens D, Kunau WH. 1989. Isolation of peroxisome-deficient mutants of Saccharomyces cerevisiae. Proceedings of the National Academy of Sciences of the United States of America 86: 5419-5423.

Esser K, Straub J. 1958. Genetische Untersuchungen an Sordaria macrospora Auersw Kompensation Und Induktion Bei Genbedingten Entwicklungsdefekten. Z Verebungsl 89: 729-746.

Farre JC, Burkenroad A, Burnett SF, Subramani S. 2013. Phosphorylation of mitophagy and pexophagy receptors coordinates their interaction with Atg8 and Atg11. EMBO reports 14: 441-449.

Farre JC, Manjithaya R, Mathewson RD, Subramani S. 2008. PpAtg30 tags peroxisomes for turnover by selective autophagy. Developmental cell 14: 365-376.

Fields S, Song O. 1989. A novel genetic system to detect protein-protein interactions. Nature 340: $245-246$. 
Fujita N, Itoh T, Omori H, Fukuda M, Noda T, Yoshimori T. 2008. The Atg16L complex specifies the site of LC3 lipidation for membrane biogenesis in autophagy. Molecular Biology of the Cell 19: 2092-2100.

Goldberg AL. 2003. Protein degradation and protection against misfolded or damaged proteins. Nature 426: 895-899.

Gould SJ, Keller GA, Hosken N, Wilkinson J, Subramani S. 1989. A conserved tripeptide sorts proteins to peroxisomes. J Cell Biol 108: 1657-1664.

Hanada T, Noda NN, Satomi Y, Ichimura Y, Fujioka Y, Takao T, Inagaki F, Ohsumi Y. 2007. The Atg12-Atg5 conjugate has a novel E3-like activity for protein lipidation in autophagy. Journal of Biological Chemistry 282: 37298-37302.

Hanada T, Ohsumi Y. 2005. Structure-function relationship of Atg12, a ubiquitin-like modifier essential for autophagy. Autophagy 1: 110-118.

Hanaoka H, Noda T, Shirano Y, Kato T, Hayashi H, Shibata D, Tabata S, Ohsumi Y. 2002. Leaf senescence and starvation-induced chlorosis are accelerated by the disruption of an Arabidopsis autophagy gene. Plant Physiology 129: 1181-1193.

Hara-Kuge S, Fujiki Y. 2008. The peroxin Pex14p is involved in LC3-dependent degradation of mammalian peroxisomes. Exp Cell Res 314: 3531-3541.

Harding TM, Morano KA, Scott SV, Klionsky DJ. 1995. Isolation and characterization of yeast mutants in the cytoplasm to vacuole protein targeting pathway. J Cell Biol 131: 591602.

He C, Klionsky DJ. 2009. Regulation mechanisms and signaling pathways of autophagy. Annu Rev Genet 43: 67-93.

Hiltunen JK, Mursula AM, Rottensteiner H, Wierenga RK, Kastaniotis AJ, Gurvitz A. 2003. The biochemistry of peroxisomal beta-oxidation in the yeast Saccharomyces cerevisiae. Fems Microbiol Rev 27: 35-64.

Hoff B, Kück U. 2005. Use of bimolecular fluorescence complementation to demonstrate transcription factor interaction in nuclei of living cells from the filamentous fungus Acremonium chrysogenum. Current genetics 47: 132-138.

Hofmann K, Bucher P. 1996. The UBA domain: a sequence motif present in multiple enzyme classes of the ubiquitination pathway. Trends Biochem Sci 21: 172-173.

Hu CD, Kerppola TK. 2003. Simultaneous visualization of multiple protein interactions in living cells using multicolor fluorescence complementation analysis. Nat Biotechnol 21: 539-545.

Hynes MJ, Murray SL, Khew GS, Davis MA. 2008. Genetic analysis of the role of peroxisomes in the utilization of acetate and fatty acids in Aspergillus nidulans. Genetics 178: 13551369.

Ichimura Y, Kirisako T, Takao T, Satomi Y, Shimonishi Y, Ishihara N, Mizushima N, Tanida I, Kominami E, Ohsumi M et al. 2000. A ubiquitin-like system mediates protein lipidation. Nature 408: 488-492.

Ichimura Y, Kumanomidou T, Sou YS, Mizushima T, Ezaki J, Ueno T, Kominami E, Yamane T, Tanaka K, Komatsu M. 2008. Structural basis for sorting mechanism of p62 in selective autophagy. The Journal of biological chemistry 283: 22847-22857.

Imazaki A, Tanaka A, Harimoto Y, Yamamoto M, Akimitsu K, Park P, Tsuge T. 2010. Contribution of peroxisomes to secondary metabolism and pathogenicity in the fungal plant pathogen Alternaria alternata. Eukaryotic cell 9: 682-694.

Ito H, Fukuda Y, Murata K, Kimura A. 1983. Transformation of intact yeast cells treated with alkali cations. Journal of Bacteriology 153: 163-168.

James P, Halladay J, Craig EA. 1996. Genomic libraries and a host strain designed for highly efficient two-hybrid selection in yeast. Genetics 144: 1425-1436. 
Janus D, Hoff B, Hofmann E, Kück U. 2007. An efficient fungal RNA-silencing system using the DsRed reporter gene. Appl Environ Microbiol 73: 962-970.

Jedd G. 2011. Fungal evo-devo: organelles and multicellular complexity. Trends in cell biology 21: 12-19.

Jedd G, Chua NH. 2000. A new self-assembled peroxisomal vesicle required for efficient resealing of the plasma membrane. Nat Cell Biol 2: 226-231.

Jenner L, Melnikov S, de Loubresse NG, Ben-Shem A, Iskakova M, Urzhumtsev A, Meskauskas A, Dinman J, Yusupova G, Yusupov M. 2012. Crystal structure of the 80S yeast ribosome. Curr Opin Struc Biol 22: 759-767.

Johansen T, Lamark T. 2011. Selective autophagy mediated by autophagic adapter proteins. Autophagy 7: 279-296.

Jones P, Binns D, Chang H-Y, Fraser M, Li W, McAnulla C, McWilliam H, Maslen J, Mitchell A, Nuka G et al. 2014. InterProScan 5: genome-scale protein function classification. Bioinformatics 30: 1236-1240.

Josefsen L, Droce A, Sondergaard TE, Sörensen JL, Bormann J, Schäfer W, Giese H, Olsson S. 2012. Autophagy provides nutrients for nonassimilating fungal structures and is necessary for plant colonization but not for infection in the necrotrophic plant pathogen Fusarium graminearum. Autophagy 8: 326-337.

Kang DH, Gho YS, Suh MK, Kang CH. 2002. Highly sensitive and fast protein detection with coomassie brilliant blue in sodium dodecyl sulfate-polyacrylamide gel electrophoresis. B Korean Chem Soc 23: 1511-1512.

Kanki T, Klionsky DJ. 2009. Atg32 is a tag for mitochondria degradation in yeast. Autophagy 5: 1201-1202.

Kanki T, Kurihara Y, Jin XL, Goda T, Ono Y, Aihara M, Hirota Y, Saigusa T, Aoki Y, Uchiumi $\mathrm{T}$ et al. 2013. Casein kinase 2 is essential for mitophagy. EMBO reports 14: 788-794.

Katarzyna ZR, Suresh S. 2016. Autophagic degradation of peroxisomes in mammals. Biochem Soc T 44: 431-440.

Kaufmann A, Beier V, Franquelim Henri G, Wollert T. 2014. Molecular mechanism of autophagic membrane-scaffold assembly and disassembly. Cell 156: 469-481.

Kawabata T, Kato A, Suzuki K, Inoue H. 2007. Neurosprora crassa RAD5 homologue, mus41, inactivation results in higher sensitivity to mutagens but has little effect on PCNAubiquitylation in response to UV-irradiation. Current genetics 52: 125-135.

Kershaw MJ, Talbot NJ. 2009. Genome-wide functional analysis reveals that infectionassociated fungal autophagy is necessary for rice blast disease. Proceedings of the National Academy of Sciences 106: 15967-15972.

Khalfan WA, Klionsky DJ. 2002. Molecular machinery required for autophagy and the cytoplasm to vacuole targeting (Cvt) pathway in S. cerevisiae. Curr Opin Cell Biol 14: 468-475.

Kiel JAKW, van der Klei IJ. 2009. Proteins involved in microbody biogenesis and degradation in Aspergillus nidulans. Fungal Genetics and Biology 46: S62-S71.

Kiel JAKW, Veenhuis M, van der Klei IJ. 2006. PEX genes in fungal genomes: Common, rare or redundant. Traffic 7: 1291-1303.

Kim I, Rodriguez-Enriquez S, Lemasters JJ. 2007. Selective degradation of mitochondria by mitophagy. Arch Biochem Biophys 462: 245-253.

Kim PK, Hailey DW, Mullen RT, Lippincott-Schwartz J. 2008. Ubiquitin signals autophagic degradation of cytosolic proteins and peroxisomes. Proc Natl Acad Sci U S A 105: 20567-20574.

Kim S-H, Moon E-K, Hong Y, Chung D-I, Kong H-H. 2015. Autophagy protein 12 plays an essential role in Acanthamoeba encystation. Experimental Parasitology 159: 46-52. 
Kimura A, Takano Y, Furusawa I, Okuno T. 2001. Peroxisomal metabolic function is required for appressorium-mediated plant infection by Colletotrichum lagenarium. Plant Cell 13: $1945-1957$.

Kimura S, Maruyama J, Kikuma T, Arioka M, Kitamoto K. 2011. Autophagy delivers misfolded secretory proteins accumulated in endoplasmic reticulum to vacuoles in the filamentous fungus Aspergillus oryzae. Biochem Bioph Res Co 406: 464-470.

Kirkin V, Lamark T, Sou YS, Bjorkoy G, Nunn JL, Bruun JA, Shvets E, McEwan DG, Clausen $\mathrm{TH}$, Wild $\mathrm{P}$ et al. 2009. A role for NBR1 in autophagosomal degradation of ubiquitinated substrates. Molecular cell 33: 505-516.

Kitamura K, Kishi-Itakura C, Tsuboi T, Sato S, Kita K, Ohta N, Mizushima N. 2012. Autophagy-related Atg8 localizes to the apicoplast of the human Malaria parasite Plasmodium falciparum. Plos One 7: e42977.

Klionsky DJ Abdalla FC Abeliovich H Abraham RT Acevedo-Arozena A Adeli K Agholme L Agnello M Agostinis P Aguirre-Ghiso JA et al. 2012. Guidelines for the use and interpretation of assays for monitoring autophagy. Autophagy 8: 445-544.

Klionsky DJ Abdelmohsen K Abe A Abedin MJ Abeliovich H Arozena AA Adachi H Adams CM Adams PD Adeli K et al. 2016. Guidelines for the use and interpretation of assays for monitoring autophagy (3rd edition). Autophagy 12: 1-222.

Klix V, Nowrousian M, Ringelberg C, Loros JJ, Dunlap JC, Pöggeler S. 2010. Functional characterization of MAT1-1-specific mating-type genes in the homothallic ascomycete Sordaria macrospora provides new insights into essential and non-essential sexual regulators. Eukaryot Cell 9: 894-905.

Komatsu M, Waguri S, Chiba T, Murata S, Iwata J, Tanida I, Ueno T, Koike M, Uchiyama Y, Kominami $\mathrm{E}$ et al. 2006. Loss of autophagy in the central nervous system causes neurodegeneration in mice. Nature 441: 880-884.

Kraft C, Deplazes A, Sohrmann M, Peter M. 2008. Mature ribosomes are selectively degraded upon starvation by an autophagy pathway requiring the Ubp3p/Bre5p ubiquitin protease. Nat Cell Biol 10: 602-610.

Kraft C, Peter M, Hofmann K. 2010. Selective autophagy: ubiquitin-mediated recognition and beyond. Nat Cell Biol 12: 836-841.

Kück U, S. P, Nowrousian M, Nolting N, Engh I. 2009. Sordaria macrospora, a model system for fungal development Springer-Verlag Berlin Heidelberg.

Kuma A, Matsui M, Mizushima N. 2007. LC3, an autophagosome marker, can be incorporated into protein aggregates independent of autophagy: caution in the interpretation of LC3 localization. Autophagy 3: 323-328.

Laemmli UK. 1970. Cleavage of structural proteins during the assembly of the head of bacteriophage T4. Nature 227: 680-685.

Lamark T, Kirkin V, Dikic I, Johansen T. 2009. NBR1 and p62 as cargo receptors for selective autophagy of ubiquitinated targets. Cell Cycle 8: 1986-1990.

Levine B, Kroemer G. 2008. Autophagy in the pathogenesis of disease. Cell 132: 27-42.

Lew RR. 2005. Mass flow and pressure-driven hyphal extension in Neurospora crassa. Microbiol-Sgm 151: 2685-2692.

Li F, Chung T, Pennington JG, Federico ML, Kaeppler HF, Kaeppler SM, Otegui MS, Vierstra RD. 2015. Autophagic recycling plays a central role in maize nitrogen remobilization. The Plant Cell 27: 1389-1408.

Li L, Wang J, Chen H, Gu Z, Wang Y, Sun G. 2016. Pex14/17, a filamentous fungi specific peroxin, is required for import of peroxisomal matrix proteins and full virulence of Magnaporthe oryzae. Molecular plant pathology.

Li WW, Li J, Bao JK. 2012. Microautophagy: lesser-known self-eating. Cellular and molecular life sciences : CMLS 69: 1125-1136. 
Liu FF, Ng SK, Lu YF, Low W, Lai J, Jedd G. 2008. Making two organelles from one: Woronin body biogenesis by peroxisomal protein sorting. J Cell Biol 180: 325-339.

Liu L, Feng D, Chen G, Chen M, Zheng QX, Song PP, Ma Q, Zhu CZ, Wang R, Qi WJ et al. 2012. Mitochondrial outer-membrane protein FUNDC1 mediates hypoxia-induced mitophagy in mammalian cells. Nature Cell Biology 14: 177-185.

Liu XM, Sun LL, Hu W, Ding YH, Dong MQ, Du LL. 2015. ESCRTs cooperate with a selective autophagy receptor to mediate vacuolar targeting of soluble cargos. Molecular cell 59: 1035-1042.

Lord KM, Read ND. 2011. Perithecium morphogenesis in Sordaria macrospora. Fungal genetics and biology : $F G$ \& B 48: 388-399.

Lynch-Day MA, Klionsky DJ. 2010. The Cvt pathway as a model for selective autophagy. Febs Lett 584: 1359-1366.

MacVicar T. 2013. Mitophagy. Essays in biochemistry 55: 93-104.

Maggio-Hall LA, Wilson RA, Keller NP. 2005. Fundamental contribution of beta-oxidation to polyketide mycotoxin production in planta. Molecular plant-microbe interactions : MPMI 18: 783-793.

Managadze D, Wurtz C, Sichting M, Niehaus G, Veenhuis M, Rottensteiner H. 2007. The peroxin PEX14 of Neurospora crassa is essential for the biogenesis of both glyoxysomes and Woronin bodies. Traffic 8: 687-701.

Marchler-Bauer A, Derbyshire MK, Gonzales NR, Lu S, Chitsaz F, Geer LY, Geer RC, He J, Gwadz M, Hurwitz DI et al. 2015. CDD: NCBI's conserved domain database. Nucleic acids research 43: 222-226.

Mardakheh FK, Auciello G, Dafforn TR, Rappoport JZ, Heath JK. 2010. Nbr1 is a novel inhibitor of ligand-mediated receptor tyrosine kinase degradation. Mol Cell Biol 30: 5672-5685.

Markham P. 1994. Occlusions of septal pores in filamentous fungi. Mycol Res 98: 1089-1106.

Maruyama JI, Juvvadi PR, Ishi K, Kitamoto K. 2005. Three-dimensional image analysis of plugging at the septal pore by Woronin body during hypotonic shock inducing hyphal tip bursting in the filamentous fungus Aspergillus oryzae. Biochem Bioph Res Co 331: 1081-1088.

Massey A, Kiffin R, Cuervo AM. 2004. Pathophysiology of chaperone-mediated autophagy. Int J Biochem Cell Biol 36: 2420-2434.

Matsumoto G, Wada K, Okuno M, Kurosawa M, Nukina N. 2011. Serine 403 phosphorylation of p62/SQSTM1 regulates selective autophagic clearance of ubiquitinated proteins. Molecular cell 44: 279-289.

Mayrhofer S, Weber JM, Pöggeler S. 2006. Pheromones and pheromone receptors are required for proper sexual development in the homothallic ascomycete Sordaria macrospora. Genetics 172: 1521-1533.

Mazure NM, Pouyssegur J. 2010. Hypoxia-induced autophagy: cell death or cell survival? Curr Opin Cell Biol 22: 177-180.

Meijer WH, van der Klei IJ, Veenhuis M, Kiel JA. 2007a. ATG genes involved in non-selective autophagy are conserved from yeast to man, but the selective Cvt and pexophagy pathways also require organism-specific genes. Autophagy 3: 106-116.

Meijer WH, van der Klei IJ, Veenhuis M, Kiel JAKW. 2007b. ATG genes involved in nonselective autophagy are conserved from yeast to man, but the selective Cvt and pexophagy pathways also require organism-specific genes. Autophagy 3: 106-116.

Metlagel Z, Otomo C, Takaesu G, Otomo T. 2013. Structural basis of ATG3 recognition by the autophagic ubiquitin-like protein ATG12. Proceedings of the National Academy of Sciences 110: 18844-18849. 
Milisav I, Suput D, Ribaric S. 2015. Unfolded protein response and macroautophagy in Alzheimer's, Parkinson's and Prion Diseases. Molecules 20: 22718-22756.

Mizushima N. 2004. Methods for monitoring autophagy. The International Journal of Biochemistry \& Cell Biology 36: 2491-2502.

Mizushima N. 2005. The pleiotropic role of autophagy: from protein metabolism to bactericide. Cell Death Differ 12 Suppl 2: 1535-1541.

-. 2015. Nbr1, a receptor for ESCRT-dependent endosomal microautophagy in fission yeast. Molecular cell 59: 887-889.

Mizushima N, Kuma A, Kobayashi Y, Yamamoto A, Matsubae M, Takao T, Natsume T, Ohsumi Y, Yoshimori T. 2003. Mouse Apg16L, a novel WD-repeat protein, targets to the autophagic isolation membrane with the Apg12-Apg5 conjugate. Journal of cell science 116: 1679-1688.

Mizushima N, Levine B, Cuervo AM, Klionsky DJ. 2008. Autophagy fights disease through cellular self-digestion. Nature 451: 1069-1075.

Mizushima N, Noda T, Ohsumi Y. 1999. Apg16p is required for the function of the Apg12pApg5p conjugate in the yeast autophagy pathway. EMBO J 18: 3888-3896.

Mizushima N, Noda T, Yoshimori T, Tanaka Y, Ishii T, George MD, Klionsky DJ, Ohsumi M, Ohsumi Y. 1998. A protein conjugation system essential for autophagy. Nature 395: 395-398.

Mizushima N, Yamamoto A, Hatano M, Kobayashi Y, Kabeya Y, Suzuki K, Tokuhisa T, Ohsumi Y, Yoshimori T. 2001. Dissection of autophagosome formation using Apg5deficient mouse embryonic stem cells. The Journal of Cell Biology 152: 657-668.

Mochida K, Oikawa Y, Kimura Y, Kirisako H, Hirano H, Ohsumi Y, Nakatogawa H. 2015. Receptor-mediated selective autophagy degrades the endoplasmic reticulum and the nucleus. Nature 522: 359-362.

Möckli N, Auerbach D. 2004. Quantitative beta-galactosidase assay suitable for highthroughput applications in the yeast two-hybrid system. Biotechniques 36: 872-876.

Montilla-Martinez M, Beck S, Klumper J, Meinecke M, Schliebs W, Wagner R, Erdmann R. 2015. Distinct pores for peroxisomal import of PTS1 and PTS2 proteins. Cell Rep 13: 2126-2134.

Motley AM, Nuttall JM, Hettema EH. 2012. Atg36 The Saccharomyces cerevisiae receptor for pexophagy. Autophagy 8: 1680-1681.

Mukaiyama H, Kajiwara S, Hosomi A, Giga-Hama Y, Tanaka N, Nakamura T, Takegawa K. 2009. Autophagy-deficient Schizosaccharomyces pombe mutants undergo partial sporulation during nitrogen starvation. Microbiology 155: 3816-3826.

Mullis KB, Faloona FA. 1987. Specific synthesis of DNA in vitro via a polymerase-catalyzed chain-reaction. Methods in Enzymology 155: 335-350.

Mumberg D, Müller R, Funk M. 1994. Regulatable promoters of Saccharomyces cerevisiae: comparison of transcriptional activity and their use for heterologous expression. Nucleic acids research 22: 5767-5768.

Munro S. 2011. The golgin coiled-coil proteins of the golgi apparatus. Csh Perspect Biol 3.

Murrow L, Malhotra R, Debnath J. 2015. ATG12-ATG3 interacts with Alix to promote basal autophagic flux and late endosome function. Nat Cell Biol 17: 300-310.

Nakatogawa H, Mochida K. 2015. Reticulophagy and nucleophagy: New findings and unsolved issues. Autophagy 11: 2377-2378.

Nazarko TY. 2014. Atg37 regulates the assembly of the pexophagic receptor protein complex. Autophagy 10: 1348-1349.

Nazarko TY, Ozeki K, Till A, Ramakrishnan G, Lotfi P, Yan MD, Subramani S. 2014. Peroxisomal Atg37 binds Atg30 or palmitoyl-CoA to regulate phagophore formation during pexophagy. J Cell Biol 204: 541-557. 
Ng SK, Liu FF, Lai JL, Low W, Jedd G. 2009. A Tether for Woronin Body Inheritance Is Associated with Evolutionary Variation in Organelle Positioning. PLoS genetics 5: 112.

Nicot AS, Lo Verso F, Ratti F, Pilot-Storck F, Streichenberger N, Sandri M, Schaeffer L, Goillot E. 2014. Phosphorylation of NBR1 by GSK3 modulates protein aggregation. Autophagy 10: 1036-1053.

Noda NN, Fujioka Y, Hanada T, Ohsumi Y, Inagaki F. 2013. Structure of the Atg12-Atg5 conjugate reveals a platform for stimulating Atg8-PE conjugation. EMBO reports 14: 206-211.

Noda NN, Inagaki F. 2015. Mechanisms of Autophagy. Annu Rev Biophys 44: 101-122.

Noda NN, Kumeta H, Nakatogawa H, Satoo K, Adachi W, Ishii J, Fujioka Y, Ohsumi Y, Inagaki F. 2008. Structural basis of target recognition by Atg8/LC3 during selective autophagy. Genes Cells 13: 1211-1218.

Noda T, Suzuki K, Ohsumi Y. 2002. Yeast autophagosomes: de novo formation of a membrane structure. Trends in cell biology 12: 231-235.

Nolting N, Bernhards Y, Pöggeler S. 2009. SmATG7 is required for viability in the homothallic ascomycete Sordaria macrospora. Fungal genetics and biology: FG \& B 46: 531-542.

Nolting N, Pöggeler S. 2006. A MADS box protein interacts with a mating-type protein and is required for fruiting-body development in the homothallic ascomycete Sordaria macrospora. Eukaryotic cell 5: 1043-1056.

Novak I, Kirkin V, McEwan DG, Zhang J, Wild P, Rozenknop A, Rogov V, Lohr F, Popovic D, Occhipinti A et al. 2010. Nix is a selective autophagy receptor for mitochondrial clearance. EMBO reports 11: 45-51.

Nowrousian M, Masloff S, Pöggeler S, Kück U. 1999. Cell differentiation during sexual development of the fungus Sordaria macrospora requires ATP citrate lyase activity. Mol Cell Biol 19: 450-460.

Nowrousian M, Stajich JE, Chu M, Engh I, Espagne E, Halliday K, Kamerewerd J, Kempken F, Knab B, Kuo HC et al. 2010. De novo assembly of a $40 \mathrm{Mb}$ eukaryotic genome from short sequence reads: Sordaria macrospora, a model organism for fungal morphogenesis. PLoS genetics 6: e1000891.

Nowrousian M, Teichert I, Masloff S, Kück U. 2012. Whole-genome sequencing of Sordaria macrospora mutants identifies developmental genes. G3 (Bethesda) 2: 261-270.

Ohsumi Y. 2001. Molecular dissection of autophagy: two ubiquitin-like systems. Nat Rev Mol Cell Biol 2: 211-216.

Okamoto K. 2014. Organellophagy: eliminating cellular building blocks via selective autophagy. J Cell Biol 205: 435-445.

Okamoto K, Kondo-Okamoto N, Ohsumi Y. 2009. A landmark protein essential for mitophagy Atg32 recruits the autophagic machinery to mitochondria. Autophagy 5: 1203-1205.

Opalinski L, Kiel JAKW, Homan TG, Veenhuis M, van der Klei IJ. 2010. Penicillium chrysogenum Pex14/17p-a novel component of the peroxisomal membrane that is important for penicillin production. Febs $J$ 277: 3203-3218.

Ossareh-Nazari B, Bonizec M, Cohen M, Dokudovskaya S, Delalande F, Schaeffer C, Van Dorsselaer A, Dargemont C. 2010. Cdc48 and Ufd3, new partners of the ubiquitin protease Ubp3, are required for ribophagy. EMBO reports 11: 548-554.

Ossareh-Nazari B, Nino CA, Bengtson MH, Lee JW, Joazeiro CAP, Dargemont C. 2014. Ubiquitylation by the Ltn1 E3 ligase protects $60 \mathrm{~S}$ ribosomes from starvation-induced selective autophagy. J Cell Biol 204: 909-917.

Otomo C, Metlagel Z, Takaesu G, Otomo T. 2013. Structure of the human ATG12 ATG5 conjugate required for LC3 lipidation in autophagy. Nat Struct Mol Biol 20: 59-66. 
Page RDM. 1996. Tree View: An application to display phylogenetic trees on personal computers. Computer applications in the biosciences : CABIOS 12: 357-358.

Pankiv S, Clausen TH, Lamark T, Brech A, Bruun JA, Outzen H, Overvatn A, Bjorkoy G, Johansen T. 2007. p62/SQSTM1 binds directly to Atg8/LC3 to facilitate degradation of ubiquitinated protein aggregates by autophagy. The Journal of biological chemistry $\mathbf{2 8 2}$ : 24131-24145.

Papinski D, Kraft C. 2016. Regulation of autophagy by signaling through the Atg1/ULK1 complex. Journal of molecular biology 428: 1725-1741.

Papinski D, Schuschnig M, Reiter W, Wilhelm L, Barnes CA, Maiolica A, Hansmann I, Pfaffenwimmer T, Kijanska M, Stoffel I et al. 2014. Early steps in autophagy depend on direct phosphorylation of Atg9 by the Atg1 kinase. Molecular cell 53: 471-483.

Peraza-Reyes L, Arnaise S, Zickler D, Coppin E, Debuchy R, Berteaux-Lecellier V. 2011. The importomer peroxins are differentially required for peroxisome assembly and meiotic development in Podospora anserina: insights into a new peroxisome import pathway. Mol Microbiol 82: 365-377.

Peraza-Reyes L, Zickler D, Berteaux-Lecellier V. 2008. The peroxisome RING-finger complex is required for meiocyte formation in the fungus Podospora anserina. Traffic 9: 19982009.

Petriv OI, Tang L, Titorenko VI, Rachubinski RA. 2004. A new definition for the consensus sequence of the peroxisome targeting signal type 2. Journal of molecular biology 341: 119-134.

Pfaffenwimmer T, Reiter W, Brach T, Nogellova V, Papinski D, Schuschnig M, Abert C, Ammerer G, Martens S, Kraft C. 2014. Hrr25 kinase promotes selective autophagy by phosphorylating the cargo receptor Atg19. EMBO reports 15: 862-870.

Pöggeler S, Kück U. 2006. Highly efficient generation of signal transduction knockout mutants using a fungal strain deficient in the mammalian ku70 ortholog. Gene 378: 1-10.

Pöggeler S, Masloff S, Hoff B, Mayrhofer S, Kück U. 2003. Versatile EGFP reporter plasmids for cellular localization of recombinant gene products in filamentous fungi. Current genetics 43: 54-61.

Pöggeler S, Masloff S, Jacobsen S, Kück U. 2000. Karyotype polymorphism correlates with intraspecific infertility in the homothallic ascomycete Sordaria macrospora. J Evolution Biol 13: 281-289.

Pöggeler S, Nowrousian M, Jacobsen S, Kück U. 1997. An efficient procedure to isolate fungal genes from an indexed cosmid library. J Microbiol Methods: 49-61.

Pöggeler S, Nowrousian M, Ringelberg C, Loros JJ, Dunlap JC, Kück U. 2006. Microarray and real-time PCR analyses reveal mating type-dependent gene expression in a homothallic fungus. Mol Gen Genomics 275: 492-503.

Pollack JK, Harris SD, Marten MR. 2009. Autophagy in filamentous fungi. Fungal genetics and biology : $F G$ \& $B$ 46: 1-8.

Radoshevich L, Murrow L, Chen N, Fernandez E, Roy S, Fung C, Debnath J. 2010. ATG12 conjugation to ATG3 regulates mitochondrial homeostasis and cell death. Cell 142: 590-600.

Rappsilber J, Ishihama Y, Mann M. 2003. Stop and go extraction tips for matrix-assisted laser desorption/ionization, nanoelectrospray, and LC/MS sample pretreatment in proteomics. Anal Chem 75: 663-670.

Reggiori F, Klionsky DJ. 2013. Autophagic processes in yeast: mechanism, machinery and regulation. Genetics 194: 341-361.

Reggiori F, Shintani T, Nair U, Klionsky DJ. 2005. Atg9 cycles between mitochondria and the pre-autophagosomal structure in yeasts. Autophagy 1: 101-109. 
Romanov J, Walczak M, Ibiricu I, Schüchner S, Ogris E, Kraft C, Martens S. 2012. Mechanism and functions of membrane binding by the Atg5-Atg12/Atg16 complex during autophagosome formation. The EMBO Journal 31: 4304-4317.

Rubinstein AD, Eisenstein M, Ber Y, Bialik S, Kimchi A. 2011. The autophagy protein Atg12 associates with antiapoptotic Bcl-2 family members to promote mitochondrial apoptosis. Molecular cell 44: 698-709.

Rubinsztein DC, Shpilka T, Elazar Z. 2012. Mechanisms of autophagosome biogenesis. Current biology : CB 22: 29-34.

Saikia S, Scott B. 2009. Functional analysis and subcellular localization of two geranylgeranyl diphosphate synthases from Penicillium paxilli. Molecular Genetics and Genomics 282: 257-271.

Sakoh-Nakatogawa M, Matoba K, Asai E, Kirisako H, Ishii J, Noda NN, Inagaki F, Nakatogawa H, Ohsumi Y. 2013. Atg12-Atg5 conjugate enhances E2 activity of Atg3 by rearranging its catalytic site. Nat Struct Mol Biol 20: 433-439.

Sambrook J, Russell DW. 2001. Molecular cloning: a laboratory manual. Cold Spring Harbor Laboratory Press.

Sawa-Makarska J, Abert C, Romanov J, Zens B, Ibiricu I, Martens S. 2014. Cargo binding to Atg19 unmasks additional Atg8 binding sites to mediate membrane-cargo apposition during selective autophagy. Nat Cell Biol 16: 425-433.

Shevchenko A, Wilm M, Vorm O, Mann M. 1996. Mass spectrometric sequencing of proteins from silver stained polyacrylamide gels. Anal Chem 68: 850-858.

Shintani T, Huang WP, Stromhaug PE, Klionsky DJ. 2002. Mechanism of cargo selection in the cytoplasm to vacuole targeting pathway. Developmental cell 3: 825-837.

Shintani T, Mizushima N, Ogawa Y, Matsuura A, Noda T, Ohsumi Y. 1999. Apg10p, a novel protein-conjugating enzyme essential for autophagy in yeast. EMBO J 18: 5234-5541.

Shoji JY, Craven KD. 2011. Autophagy in basal hyphal compartments: a green strategy of great recyclers. Fungal Biol Rev 25: 79-83.

Shoji JY, Kikuma T, Arioka M, Kitamoto K. 2010. Macroautophagy-mediated degradation of whole nuclei in the filamentous fungus Aspergillus oryzae. Plos One 5.

Soundararajan S, Jedd G, Li XL, Ramos-Pamplona M, Chua NH, Naqvi NI. 2004. Woronin body function in Magnaporthe grisea is essential for efficient pathogenesis and for survival during nitrogen starvation stress. Plant Cell 16: 1564-1574.

Southern EM. 1975. Detection of specific sequences among DNA fragments separated by gel electrophoresis. Journal of molecular biology 98: 503-517.

Stolz A, Ernst A, Dikic I. 2014. Cargo recognition and trafficking in selective autophagy. Nat Cell Biol 16: 495-501.

Sugawara K, Suzuki NN, Fujioka Y, Mizushima N, Ohsumi Y, Inagaki F. 2004. The crystal structure of microtubule-associated protein light chain 3, a mammalian homologue of Saccharomyces cerevisiae Atg8. Genes Cells 9: 611-618.

Suzuki H, Tabata K, Morita E, Kawasaki M, Kato R, Dobson RC, Yoshimori T, Wakatsuki S. 2014. Structural basis of the autophagy-related LC3/Atg13 LIR complex: recognition and interaction mechanism. Structure 22: 47-58.

Suzuki K, Akioka M, Kondo-Kakuta C, Yamamoto H, Ohsumi Y. 2013. Fine mapping of autophagy-related proteins during autophagosome formation in Saccharomyces cerevisiae. Journal of cell science 126: 2534-2544.

Suzuki K, Kirisako T, Kamada Y, Mizushima N, Noda T, Ohsumi Y. 2001. The preautophagosomal structure organized by concerted functions of APG genes is essential for autophagosome formation. EMBO J 20: 5971-5981.

Suzuki K, Ohsumi Y. 2007. Molecular machinery of autophagosome formation in yeast, Saccharomyces cerevisiae. Febs Lett 581: 2156-2161. 
Suzuki K, Ohsumi Y. 2010. Current knowledge of the pre-autophagosomal structure (PAS). Febs Lett 584: 1280-1286.

Suzuki NN, Yoshimoto K, Fujioka Y, Ohsumi Y, Inagaki F. 2005. The crystal structure of plant ATG12 and its biological implication in autophagy. Autophagy 1: 119-126.

Svenning S, Lamark T, Krause K, Johansen T. 2011. Plant NBR1 is a selective autophagy substrate and a functional hybrid of the mammalian autophagic adapters NBR1 and p62/SQSTM1. Autophagy 7: 993-1010.

Swinkels BW, Gould SJ, Bodnar AG, Rachubinski RA, Subramani S. 1991. A Novel, cleavable peroxisomal targeting signal at the amino-terminus of the Rat 3-ketoacyl-coA thiolase. Embo Journal 10: 3255-3262.

Tanabe Y, Maruyama J, Yamaoka S, Yahagi D, Matsuo I, Tsutsumi N, Kitamoto K. 2011. Peroxisomes are involved in biotin biosynthesis in Aspergillus and Arabidopsis. The Journal of biological chemistry 286: 30455-30461.

Tanaka C, Tan LJ, Mochida K, Kirisako H, Koizumi M, Asai E, Sakoh-Nakatogawa M, Ohsumi Y, Nakatogawa H. 2014. Hrr25 triggers selective autophagy-related pathways by phosphorylating receptor proteins. J Cell Biol 207: 91-105.

Tanida I, Mizushima N, Kiyooka M, Ohsumi M, Ueno T, Ohsumi Y, Kominami E. 1999. Apg7p/Cvt2p: A novel protein-activating enzyme essential for autophagy. Mol Biol Cell 10: $1367-1379$.

Teichert I, Nowrousian M, Pöggeler S, Kück U. 2014. The filamentous fungus Sordaria macrospora as a genetic model to study fruiting body development. Adv Genet 87: 199244.

Teichert I, Wolff G, Kuck U, Nowrousian M. 2012. Combining laser microdissection and RNAseq to chart the transcriptional landscape of fungal development. BMC Genomics 13: 511.

Tenney K, Hunt I, Sweigard J, Pounder JI, McClain C, Bowman EJ, Bowman BJ. 2000. hex-1, a gene unique to filamentous fungi, encodes the major protein of the Woronin body and functions as a plug for septal pores. Fungal Genetics and Biology 31: 205-217.

Thompson JD, Gibson TJ, Plewniak F, Jeanmougin F, Higgins DG. 1997. The CLUSTAL_X windows interface: flexible strategies for multiple sequence alignment aided by quality analysis tools. Nucleic acids research 25: 4876-4882.

Towbin H, Staehelin T, Gordon J. 1979. Electrophoretic transfer of proteins from polyacrylamide gels to nitrocellulose sheets: procedure and some applications. Proc Natl Acad Sci U S A 76: 4350-4354.

Tsukada M, Ohsumi Y. 1993. Isolation and characterization of autophagy-defective mutants of Saccharomyces cerevisiae. Febs Lett 333: 169-174.

Tucker CL, Peteya LA, Pittman AM, Zhong J. 2009. A genetic test for yeast two-hybrid bait competency using RanBPM. Genetics 182: 1377-1379.

Van Dyke N, Baby J, Van Dyke MW. 2006. Stm1p, a ribosome-associated protein, is important for protein synthesis in Saccharomyces cerevisiae under nutritional stress conditions. Journal of molecular biology 358: 1023-1031.

Voigt O. 2012. The influence of autophagy on the fruiting-body development of the filamentous fungus Sordaria macrospora. Dissertation Georg-August-Universität Göttingen.

Voigt O, Herzog B, Jakobshagen A, Pöggeler S. 2013. bZIP transcription factor SmJLB1 regulates autophagy-related genes Smatg8 and Smatg4 and is required for fruiting-body development and vegetative growth in Sordaria macrospora. Fungal genetics and biology : $F G \& B$ 61: 50-60.

-. 2014. Autophagic kinases SmVPS34 and SmVPS15 are required for viability in the filamentous ascomycete Sordaria macrospora. Microbiol Res 169: 128-138. 
Voigt O, Pöggeler S. 2013a. Autophagy genes Smatg8 and Smatg4 are required for fruitingbody development, vegetative growth and ascospore germination in the filamentous ascomycete Sordaria macrospora. Autophagy 9: 33-49.

-. 2013b. Self-eating to grow and kill: autophagy in filamentous ascomycetes. Appl Microbiol Biotechnol 97: 9277-9290.

Waliullah TM, Yeasmin AM, Kaneko A, Koike N, Terasawa M, Totsuka T, Ushimaru T. 2016. Rim15 and Sch9 kinases are involved in induction of autophagic degradation of ribosomes in budding yeast. Bioscience, biotechnology, and biochemistry: 1-4.

Walkey DGA, Harvey R. 1966. Studies of ballistics of ascospores. New Phytol 65: 59-67.

Wanders RJA, Waterham HR. 2006. Biochemistry of mammalian peroxisomes revisited. Annu Rev Biochem 75: 295-332.

Warner JR. 1999. The economics of ribosome biosynthesis in yeast. Trends Biochem Sci 24: 437-440.

Watanabe Y, Noda NN, Kumeta H, Suzuki K, Ohsumi Y, Inagaki F. 2010. Selective transport of alpha-mannosidase by autophagic pathways structural basis for cargo recognition by Atg19 and Atg34. Journal of Biological Chemistry 285: 30026-30033.

Wen X, Klionsky DJ. 2016. An overview of macroautophagy in yeast. Journal of molecular biology: 1681-1699.

Werner A, Herzog B, Frey S, Pöggeler S. 2016. Autophagy-associated protein SmATG12 is required for fruiting-body formation in the filamentous ascomycete Sordaria macrospora. Plos One 11: 1-21.

Woronin M. 1864. Zur Entwicklungsgeschichte des Ascobolus pulcherrimus Cr. und einiger Pezizen Abh. Sekenb. Naturforsch 5: 333-344.

Yamashita SI, Abe K, Tatemichi Y, Fujiki Y. 2014. The membrane peroxin PEX3 induces peroxisome-ubiquitination-linked pexophagy. Autophagy 10: 1549-1564.

Yamazaki-Sato H, Tanida I, Ueno T, Kominami E. 2003. The carboxyl terminal 17 amino acids within Apg7 are essential for Apg8 lipidation, but not for Apg12 conjugation. Febs Lett 551: 71-77.

Yan Y, Backer JM. 2007. Regulation of class III (Vps34) PI3Ks. Biochem Soc T 35: 239-241.

Yang Z, Huang J, Geng J, Nair U, Klionsky DJ. 2006. Atg22 recycles amino acids to link the degradative and recycling functions of autophagy. Mol Biol Cell 17: 5094-5104.

Yao ZY, Delorme-Axford E, Backues SK, Klionsky DJ. 2015. Atg41/Icy2 regulates autophagosome formation. Autophagy 11: 2288-2299.

Yorimitsu T, Klionsky DJ. 2005a. Atg11 links cargo to the vesicle-forming machinery in the cytoplasm to vacuole targeting pathway. Molecular Biology of the Cell 16: 1593-1605.

-. 2005b. Autophagy: molecular machinery for self-eating. Cell Death Differ 12: 1542-1552.

Young PG, Bartel B. 2016. Pexophagy and peroxisomal protein turnover in plants. Bba-Mol Cell Res 1863: 999-1005.

Zaffagnini G, Martens S. 2016. Mechanisms of selective autophagy. Journal of molecular biology 428: 1714-1724.

Zhang H, Chen JA, Wang YQ, Peng L, Dong XX, Lu Y, Keating AE, Jiang TJ. 2009. A computationally guided protein-interaction screen uncovers coiled-coil interactions involved in vesicular trafficking. Journal of molecular biology 392: 228-241.

Zhou J, Wang J, Cheng Y, Chi YJ, Fan B, Yu JQ, Chen Z. 2013. NBR1-mediated selective autophagy targets insoluble ubiquitinated protein aggregates in plant stress responses. PLoS genetics 9: e1003196.

Zhou J, Wang J, Yu JQ, Chen ZX. 2014a. Role and regulation of autophagy in heat stress responses of tomato plants. Front Plant Sci 5. 
Zhou J, Zhang Y, Qi JX, Chi YJ, Fan BF, Yu JQ, Chen ZX. 2014b. E3 ubiquitin ligase CHIP and NBR1-mediated selective autophagy protect additively against proteotoxicity in plant stress responses. PLoS genetics $\mathbf{1 0}$.

Zientara-Rytter K, Lukomska J, Moniuszko G, Gwozdecki R, Surowiecki P, Lewandowska M, Liszewska F, Wawrzynska A, Sirko A. 2011. Identification and functional analysis of Joka2, a tobacco member of the family of selective autophagy cargo receptors. Autophagy 7: 1145-1158. 


\section{Appendix}

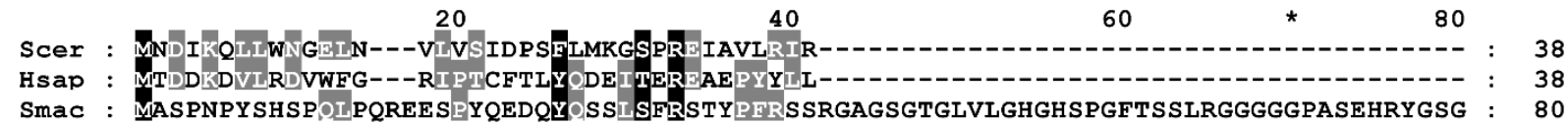

$\begin{array}{llccc} & 100 & 120 & 140 & 160\end{array}$

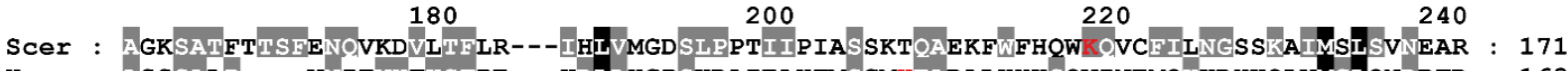

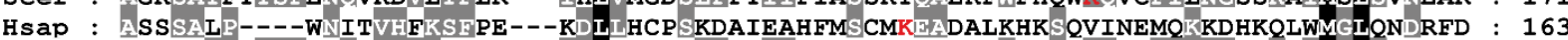

Smac : TAYYGIPCSSFHHEEVHLRNLSVGLLVDLYQPSEMPWRLVGDGDGWDICDTEMNSAKEADFIRNGNAKRIMGLSKEHTT : 240

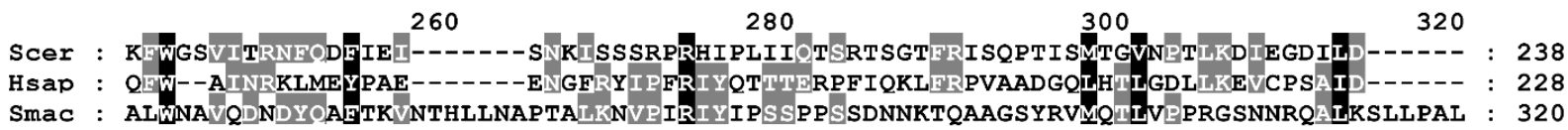

Scer : VKE GING WVMVICOGIFIPWHMLIYDLYSKLRSFD GFLYIMLVPIKGGDKASSEL : 294

Hsap : PEDGEKKNN--OVMIHGI PMLETPLOWLSEHLSY PDNELHISIIPQPTD------ : : 275

Smac : FPSSRDPVLANVILGAPVPELAPLELMRDAAY PDGWLCIIVVLI------- : 366

Supplement 1: Multiple-sequence alignment of ATG5 orthologs from Homo sapiens, Saccharomyces cerevisiae and Sordaria macrospora.

ClustalX alignment was created using the following sequences: Smac, S. macrospora (XP_003347373.1), Scer, S. cerevisiae (DAA11286.1) and Hsap, H. sapiens (Q9H1Y0.2). Amino acids which are conserved in all proteins, are shaded in black; residues conserved in two of three sequences are shaded in grey. The conserved lysine residue (Smac Lys 218, Scer Lys 149, Hsap Lys 130) which forms the conjugate with the C-terminal SmATG12 glycine residue is marked in red. 

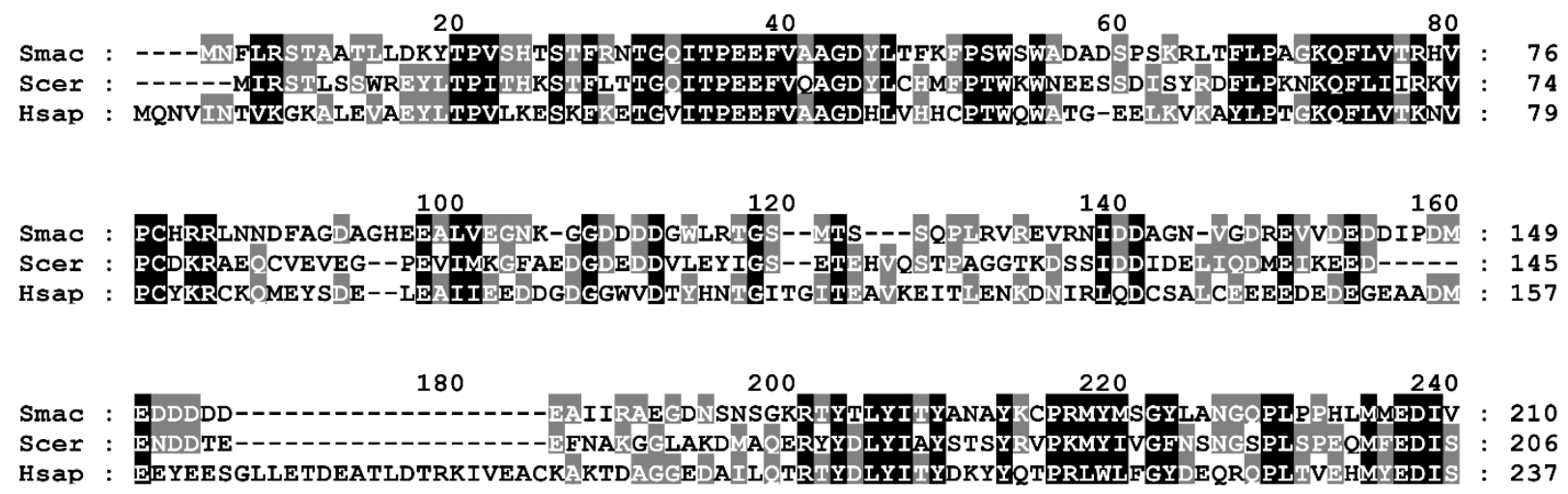

Smac : GDYKDETVTLEDFPFESHSVKMASVHPCRHASVMKTLLDRADAALKLRREKMKAGQGSGS POGMEGIVDEINKLDVSGAH : 290

Scer : ADYRTKTATIEKLPFYKNSVLSVS I H PCKHANVMKILLDKVRVVRQRRRKELQE----- BOELDGVG---------- : 268

Hsap : QDHVKKTVTIENHPHLP-PPPMCSVHPCRHAEVMKKI IETVAEGGGELGVHMYP----

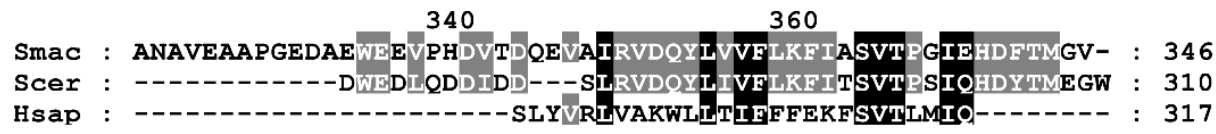

Supplement 2: Multiple-sequence alignment of ATG3 orthologs from Sordaria macrospora, Saccharomyces cerevisiae and Homo sapiens.

ClustalX alignment was created using the following sequences: Smac, S. macrospora (F7VSU2), Scer S. cerevisiae (EWG83474.1) and Hsap, H. sapiens (AAH02830.1). Amino acids, which are conserved in all proteins, are shaded in black; residues conserved in two of three sequences are shaded in grey. The conserved lysine residue (Smac Lys 216, Scer Lys 212, Hsap Lys 243) which forms the conjugate with the C-terminal ATG12 glycine residue is marked in red. 
A conserved region (CR) basidiomycetes, ascomycetes

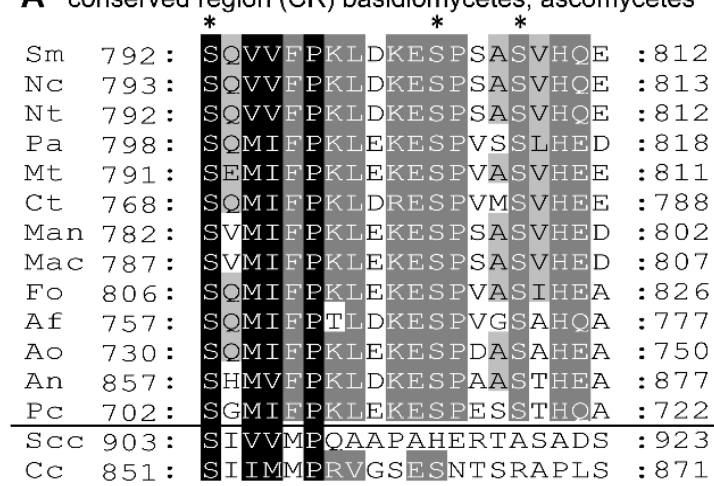

B LIR motif basidiomycetes, ascomycetes

\begin{tabular}{|c|c|c|c|c|}
\hline $\mathrm{Sm}$ & $841:$ & MTDEE & YDILDASDEESV & : 858 \\
\hline $\mathrm{NC}$ & $845:$ & MTDEE & YDILDASDEESV & : 862 \\
\hline Nt & $844:$ & MTDEE & YDILDASDEESV & $: 861$ \\
\hline $\mathrm{Pa}$ & $848:$ & MTDEE & YDILDASDEEYS & : 865 \\
\hline Mt & $842:$ & LTDE巨 & Y DVLDASDEEFN & $: 859$ \\
\hline Ct & $826:$ & LTDEE & YDVLDASDEEFS & $: 843$ \\
\hline Man & 833: & LT D & YDILDASDEEFL & $: 850$ \\
\hline Mac & $838:$ & LTDE E & YDILDASDEEFL & $: 855$ \\
\hline Fo & 854: & MTDEE & YDILDASDEEYL & : 871 \\
\hline$A f$ & $815:$ & LTDEE & Y DVLDASDQDYL & $: 832$ \\
\hline Ao & $787:$ & LTDEE & YDILDASDQEYM & $: 804$ \\
\hline An & $777:$ & LTDEE & YDILDASDQEFL & $: 794$ \\
\hline $\mathrm{PC}$ & $761:$ & LTDEE & YDVLDASDOEYL & $: 778$ \\
\hline $\mathrm{SCC}$ & 965: & & YVVLYDEVSSED & $: 982$ \\
\hline $\mathrm{Cc}$ & $952:$ & $S P N P D$ & YVLLFDDSSDSD & $: 968$ \\
\hline
\end{tabular}

Supplement 3: Alignment of single domains of NBR1 homologs in different species.

(A) Alignment of the conserved region at the $\mathrm{C}$-terminus of ascomycetes and basidiomycetes. Asterisks label the three conserved serine (S) residues in ascomycetes. Ascomycetes: Sm, Sordaria macrospora (XP_003346367); Nc, Neurospora crassa (XP_011394647); Nt, Neurospora tetrasperma (XP_009847604); Pa, Podospora anserina (XP_001912058); Mt, Myceliophthora thermophile (MYCTH_2302632); Ct, Chaetomium thermophilum (XP_006696593); Man, Metarhizium anisopliae (EFY98453); Mac, Metarhizium acridum (EFY92904); Fo, Fusarium oxysporum (EMT63555); Af, Aspergillus fumigatus (XP_755022); Ao, Aspergillus oryzae (XP_001824430); An, Aspergillus nidulans (XP_661032); Pc, Penicillium chrysogenum (XP_002563338). Basidiomycetes: Scc, Schizophyllum commune (XP_003038660); Cc, Coprinopsis cinerea (XP_001829200). (B) Alignment of the LIR motif at the C-terminal part with the same species as in (A). Asteriks label the conserved $\left(\mathrm{W} / \mathrm{Y} / \mathrm{F}_{0}\right)$ and $\left(\mathrm{L} / \mathrm{I} / \mathrm{V}_{3}\right)$ side. Amino acids, which are conserved in all proteins, are shaded in black; residues conserved in two of three sequences are shaded in grey. 


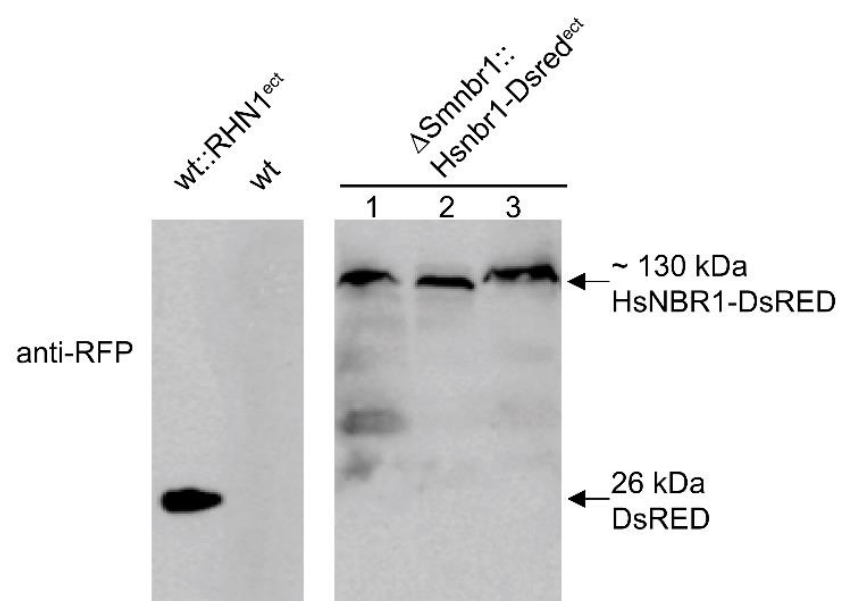

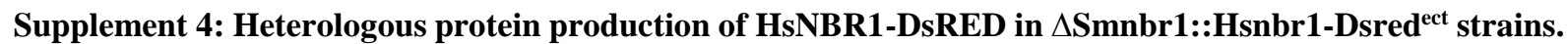
Expression of an Hsnbrl-Dsred fusion construct in $\Delta$ Smnbr1 mutant was determined by Western blotting using a monoclonal anti-RFP antibody. Three representive transformants are shown. Wt and a strain expressing free DsRED (wt::RHN1 ${ }^{\text {ect }}$ ) served as controls. The strains were grown for $3 \mathrm{~d}$ in BMM complex medium. The crude protein extracts analyzed were prepared from strains $\Delta$ Smnbr1::Hsnbr1-Dsred ${ }^{\text {ect }}$, wt::RHN1 ${ }^{\text {ect }}$ and the untransformed wt strain. 


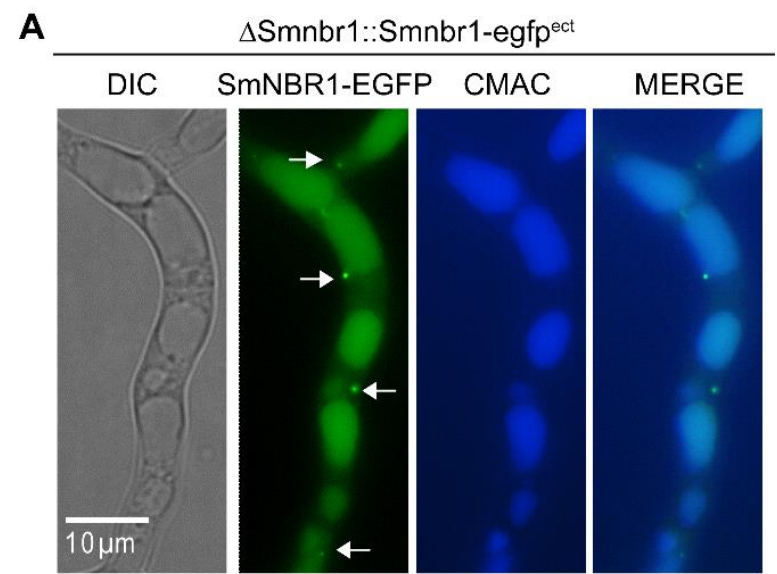

B $\Delta$ Smnbr1::egfpect

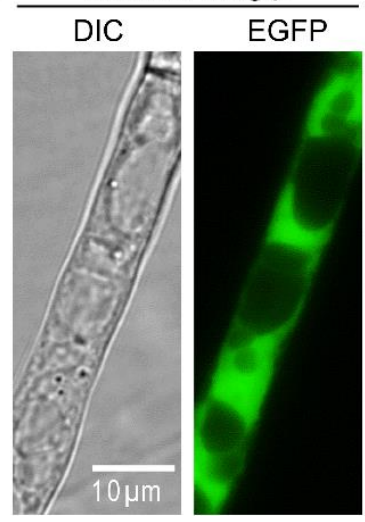

Supplement 5: Fluorescence localization of SmNBR1-EGFP in the complemented $\Delta$ Smnbr1 strain.

(A) Analysis of the strain $\Delta$ Smnbr1::Smnbr1-egfpect carrying the plasmid pSmnbr1-egfp. SmNBR1-EGFP localizes to vacuoles and autophagosomes which are indicated by arrows. Vacuoles were stained with CMAC. (B) Localization of free EGFP in the $\Delta$ Smnbr 1 strain. DIC, differential interference contrast. Scale bars are indicated. 

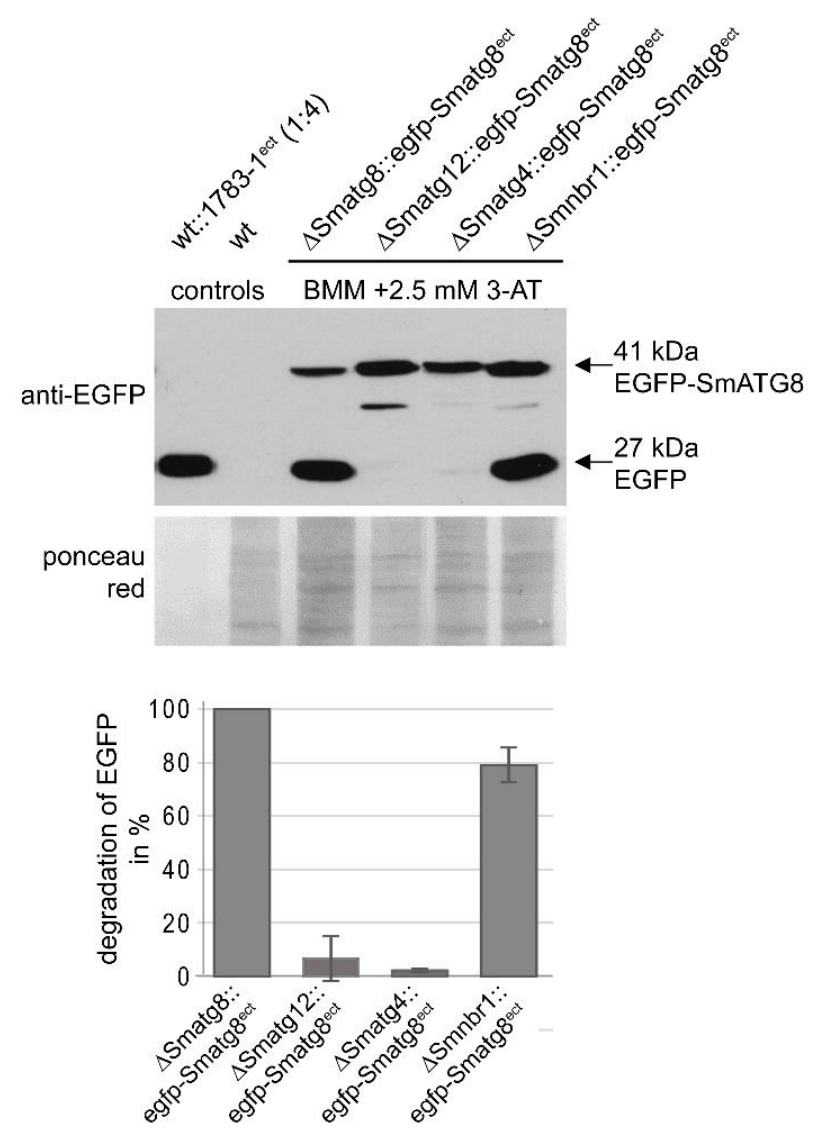

Supplement 6: Quantitative Western experiment of vacuolar degradation of EGFP-SmATG8 under aminoacid starvation condition in different autophagy mutants.

Expression of an EGFP-SmATG8 fusion construct in different autophagy mutants was determined by Western blotting using a monoclonal anti-EGFP antibody. The strains were grown for $3 \mathrm{~d}$ in BMM complex medium supplemented with $2.5 \mathrm{mM} 3-\mathrm{AT}$. Wt and a strain expressing free EGFP served as controls and were grown for 3 $\mathrm{d}$ in BMM complex medium. The crude protein extracts analyzed were prepared from strains $\Delta$ Smatg8::egfpSmatg8 ${ }^{\text {ect }}, \Delta$ Smatg12::egfp-Smatg8 ${ }^{\text {ect }}, \Delta$ Smatg4::egfp-Smatg8 ${ }^{\text {ect }}, \Delta$ Smnbr1::egfp-Smatg8 $8^{\text {ect }}$, wt::1783-1 ${ }^{\text {ect }}$ and the untransformed wt strain. Ponceau staining served as loading control to check the protein levels. The program ImageJ was used to quantify the ratio between cleaved EGFP and the EGFP-SmATG8 fusion protein. The cleavage of EGFP in the complemented strain $\Delta$ Smatg8::egfp-Smatg8 ${ }^{\text {ect }}$ was set to $100 \%$. One of three independent experiments is shown. Error bars are indicated. 
A wt::Smrpl25-egfpect

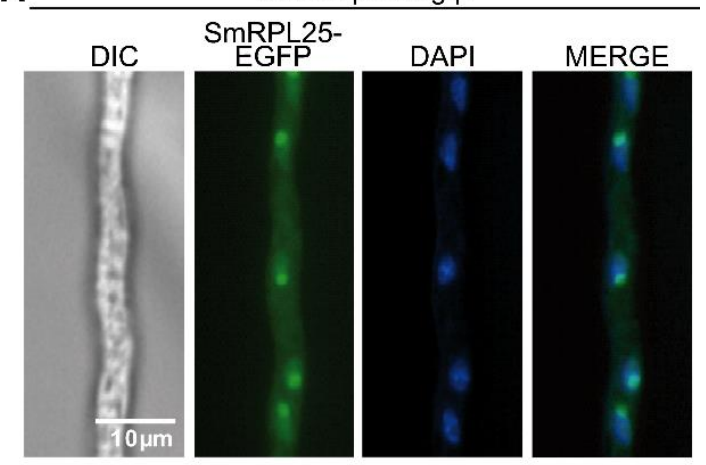

B wt::Smrps19-egfpect
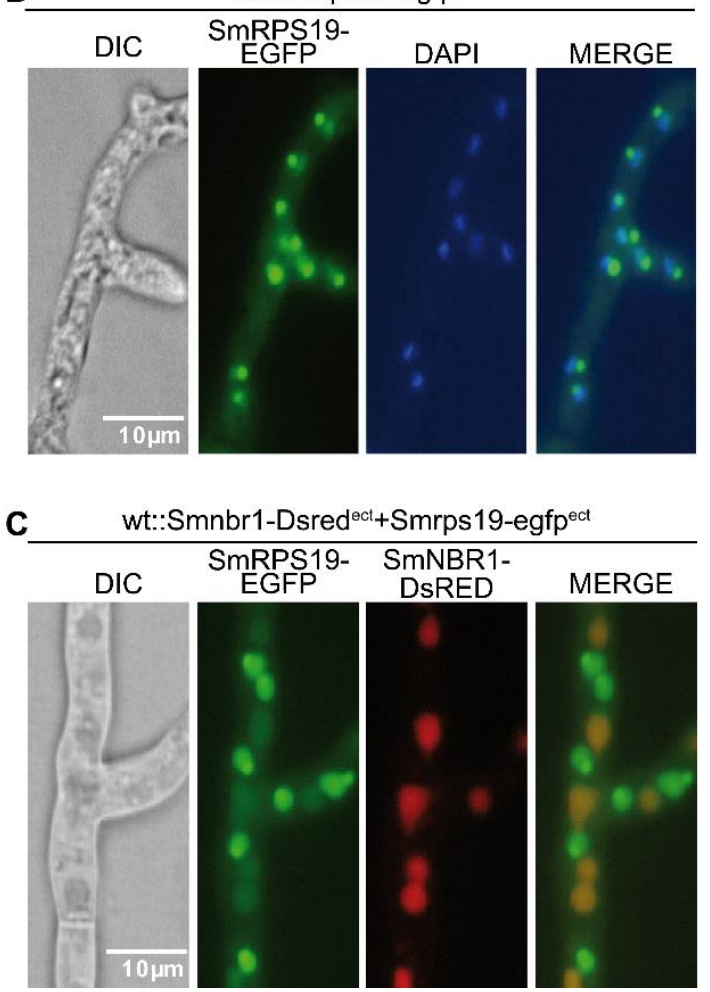

Supplement 7: Localization studies of SmRPL25-EGFP and SmRPS19-EGFP.

(A) Analysis of wt strain carrying the plasmid pSmrpl25-egfp after 24h growth on BMM medium. SmRPL25EGFP localizes to the nucleus and cytoplasm. Nuclei were stained with DAPI. (B) Analysis of wt strain carrying the plasmid pSmrps19-egfp after 24h growth on BMM medium. SmRPS19-EGFP localizes to the nucleus and cytoplasm. Nuclei were stained with DAPI. (C) Analysis of wt strain carrying the plasmid pSmrps19-egfp and pSmnbr1-Dsred after 48h growth on BMM medium. Both proteins co-localize to the vacuoles. DAPI, 4',6Diamidino-2-Phenylindole; DIC, differential interference contrast. Scale bars are indicated. Merged images are shown. 


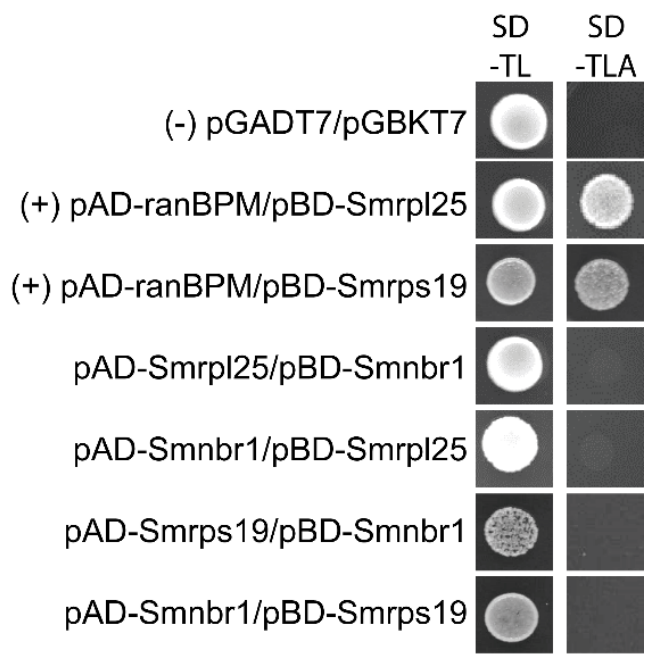

Supplement 8: Yeast-two hybrid assay of SmRPL25 and SmRPS19 with the cargo receptor SmNBR1.

Full-length cDNAs of Smrpl25, Smrps19 and Smnbrl were used to generate Gal4-DNA binding (BD) and Gal4activation domain (AD) plasmids. Yeast strains Y187 and AH109 were transformed and mated in different combinations. The mated yeast cells were grown on SD minimal medium -trp and -leu (SD-TL) for selection of the plasmids. SD medium lacking -trp, -leu, -ade (SD-TLA) was used for interaction studies. Each drop assay was performed in 10 -fold serial dilutions $\left(10^{-1}-10^{-4}\right)$. Here, the growth of the first dilution is shown. As a negative control (-), the query vectors pGADT7/pGBKT7, and for positive controls (+) the pAD-ranBPM/pBD-Smrpl25 or pBD-Smrps 19 were used. RanBPM can be used as positive control because it is able interact with the Gal4-BD (Tucker et al. 2009). 


\section{Danksagung}

Herzlichen Dank, liebe Frau Prof. Dr. Stefanie Pöggeler. Sie als meine Doktormutter haben immer ein offenes Ohr für meine Anliegen und Probleme gehabt und mir Mut verliehen, wenn es einmal nicht so gut gelaufen ist für mich. Ihr Interesse am Fortschreiten der Forschungsarbeit hat mir immer wieder neuen Anstoß gegeben, noch motivierter zu arbeiten. Ich danke Ihnen für die Möglichkeit meine Promotion in ihrer Arbeitsgruppe absolvieren zu können und das damit entgegengebrachte Vertrauen.

Ich danke Herrn Prof. Dr. Gerhard Braus und Prof. Dr. Michael Thumm, für die wissenschaftliche Betreuung sowie den hilfreichen Diskussionen und Ratschlägen als Mitglieder meines Thesis Committees.

Des Weiteren möchte ich mich bei Dr. Oliver Valerius für die Zusammenarbeit an einigen Projekten bedanken.

Mein außerordentlicher Dank gilt Dr. Britta Herzog, die mehrfache Durchsicht dieser Abhandlung, ihre kritischen Betrachtungen und ihre Anmerkungen haben zur Anfertigung und Vollendung meiner Dissertation bei getragen. Vor allem aber wegen ihrem moralischen Beistand und dem menschlichen Halt, gebührt ihr hier mein besonderer Dank. Sie ist nicht nur eine Kollegin sondern über die Zeit auch zu einer guten Freundin geworden.

Dankbar anerkennen will ich mich auch bei Gertrud Stahlhut, ihre immer freundliche und uneingeschränkte Bereitschaft mir ihr Laborwissen weiterzugeben, hat meine Leistungen im Labor gestärkt. Ihr danke ich für die ausgezeichnete und akribische Hilfe bei meinen Experimenten, aber auch für die privaten Gespräche und Ratschläge als „Labormutti“.

Ein ganz besonderer Dank geht an meine Mitdoktorandin Eva Reschka, zusammen haben wir unser Masterstudium und unsere Promotion durchlebt mit allen Höhen und Tiefen.

Allen ehemaligen Mitarbeitern und Studenten die an meinem Projekt mit gearbeitet haben, gilt herzlicher Dank. Besonders erwähnen möchte ich Silvia Carrillo-Castellon die als Laborgehilfin ein sehr gutes Arbeiten ermöglicht hat. 
Allen meinen lieben Freunden danke ich für die Ausdauer, Ruhe und Geduld, womit sie mir stets zur Seite standen und mich immer wieder aufgemuntert haben. Ihr habt mich im richtigen Moment abgelenkt um wieder Kraft zu tanken und einen klaren Blick zu bekommen.

Eine herausragende Stellung in jeglicher Hinsicht nimmt meine Familie ein doch ganz besonders meine Eltern und mein Ehemann Sebastian. Ohne Ihren Beistand wäre diese Arbeit nicht zu dem Werk geworden, welches sie heute ist. Vielen Dank für eure Unterstützung über den gesamten Zeitraum der Promotion und des Studiums, gerade aber in der Endphase in der es viele stressige und erschöpfende Momente gab. 


\section{Curriculum vitae}

Antonia Werner geb. Jakobshagen

Georg-August University Göttingen

Institute of Microbiology and Genetics

Department of Genetics of Eukaryotic Microorganisms

Grisebachstraße 8

Göttingen, Germany

E-mail: ajakobs1@gwdg.de

Date of birth: January $10^{\text {th }} 1988$

Place of birth: Leinefelde

Nationality: German

\section{$\underline{\text { Education }}$}

04/2013-present

$\mathrm{PhD}$ student in the GGNB program Microbiology and Biochemistry, Georg-August University Göttingen, Department of Genetics of Eukaryotic Microorganisms, Title of the thesis: "The interplay of SmNBR1 and SmATG8 in selective autophagy of the filamentous fungus Sordaria macrospora" Thesis Committee: Prof. Dr. Stefanie Pöggeler, Prof. Dr. Gerhard Braus, Prof. Dr. Michael Thumm

11/2012-03/2013 Scientific assistant, Georg-August University Göttingen, Department of Genetics eukaryotic Microorganisms

09/2012 Master thesis, Georg-August University Göttingen, Department of Genetics of Eukaryotic Microorganisms, Title of the thesis: "Functional analysis of the putative autophagy receptor SmNBR1 and the autophagic protein SmATG12 of the filamentous ascomycete Sordaria macrospora"

10/2010-09/2012 Master student in master program Molecular Biology, Georg-August University Göttingen

09/2010

Bachelor thesis, Georg-August University Göttingen, Department of Molecular Microbiology and Genetics, Title of the thesis: "Characterization of the Gcn4p Hac1p-interplay in the yeast Saccharomyces cerevisiae" 
10/2007-09/2010 Bachelor student in Molekulare Biowissenschaften, Georg-August University Göttingen

08/2004-07/2007 Berufliches Gymnasium Mühlhausen (Abitur)

$\underline{\text { Talks }}$

$10 / 2015$

07/2013

VAAM 2015 Berlin

"The selective autophagy cargo receptor SmNBR1 in the ascomycete Sordaria macrospora"

Retreat of the GGNB program "Microbiology and Biochemistry"

"The selective autophagy cargo receptor SmNBR1"

\section{Posters}

$12 / 2016$

$03 / 2015$

$10 / 2014$

$4^{\text {th }}$ GGNB Science Day at the Georg-August University Göttingen, Germany

"The selective autophagy cargo receptor SmNBR1 in the ascomycete Sordaria macrospora"

$28^{\text {th }}$ Fungal Genetics Conference in Asilomar, Kalifornien, USA

"The selective autophagy cargo receptor SmNBR1 in the ascomycete Sordaria macrospora“

VAAM 2014 Dresden, Germany

"Selective autophagy in the filamentous fungus Sordaria macrospora"

\section{Publications}

Antonia Werner, Britta Herzog, Stefan Frey, Stefanie Pöggeler (2016). Autophagy-associated protein SmATG12 is required for fruiting-body formation in the filamentous ascomycete Sordaria macrospora. PLOS ONE/DOI: 10.1371/journal.pone.0157960.

Daniela Heine, Linda Petereit, Marcel R. Schumann, Diana Patzelt, Leila Rachid, Ulrike Brandt, Antonia Werner, Stefanie Pöggeler, André Fleißner (2016). The tetraspanin TSP3 of Neurospora crassa is a vacuolar membrane protein and shares characteristics with IDI proteins. Mycologia: 10.385215-225. 
Oliver Voigt, Britta Herzog, Antonia Jakobshagen, Stefanie Pöggeler (2013). Autophagic kinases SmVPS34 and SmVPS15 are required for viability in the filamentous ascomycete Sordaria macrospora. Microbiol Res: 10.1016/j.micres.2013.07.012.

Oliver Voigt, Britta Herzog, Antonia Jakobshagen, Stefanie Pöggeler (2013). bZIP transcription factor SmJLB1 regulates autophagy-related genes Smatg8 and Smatg4 and is required for fruiting-body development and vegetative growth in Sordaria macrospora. Fungal Genetics and Biology: 10.1016/j.fgb.2013.09.006.

Britta Herzog, Blaga Popova, Antonia Jakobshagen, Hedieh Shahpasandzadeh, Gerhard Braus (2013). Mutual cross talk between the regulators Hac1 of the unfolded protein response and Gen4 of the general amino acid control of Saccharomyces cerevisiae. Eukaryotic Cell $12,1142-1154$. 